\title{
GATING OF PERMANENT MOLDS FOR ALUMINUM CASTINGS
}

\section{Final Technical Report}

\author{
David Schwam \\ John F. Wallace \\ Tom Engle \\ Qingming Chang \\ Department of Materials Science \\ Case Western Reserve University \\ Cleveland, Ohio
}

Work Performed Under Contract DE-FC07-01ID13983

US Department of Energy

Assistant Secretary for

Energy Efficiency and Renewable Energy

Washington DC

January 2004 


\section{TABLE OF CONTENTS}

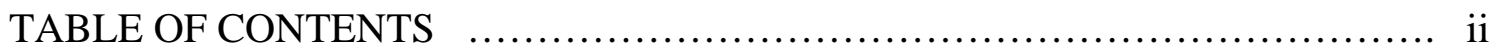

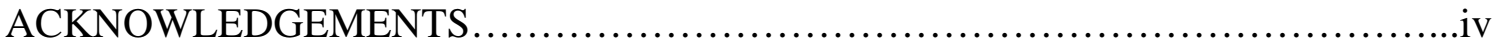

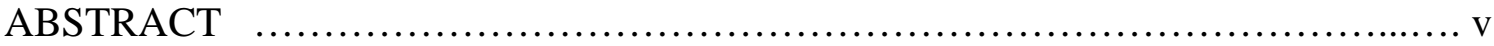

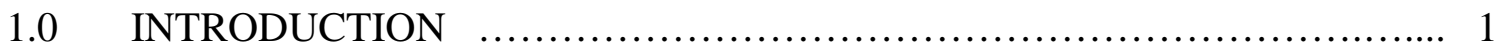

$1.1 \quad$ Permanent Vertical Mold Aluminum Casting ........................... 1

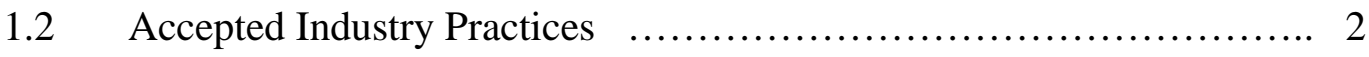

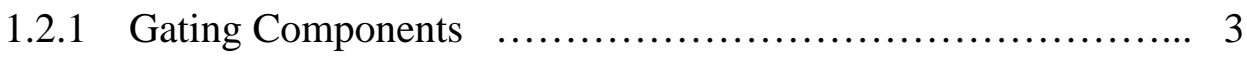

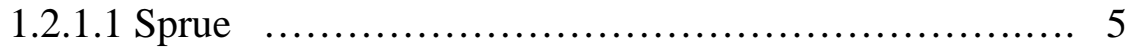

1.2.1.2 Sprue-well ......................................... 6

1.2.1.3 Feeder .................................................... 8

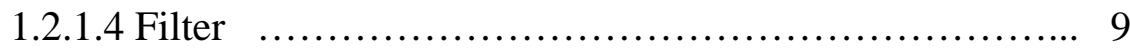

1.2.1.5 Runner ................................................. 10

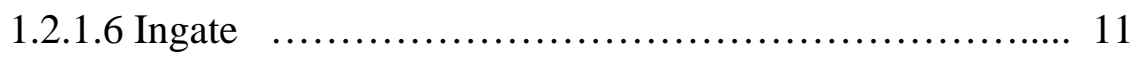

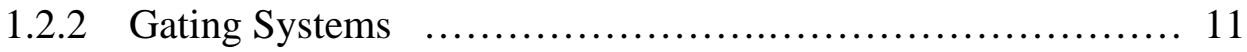

1.2.2.1 Top-feeding ....................................... 11

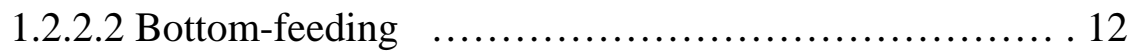

1.2.2.3 Side-feeding $\quad$.............................................12

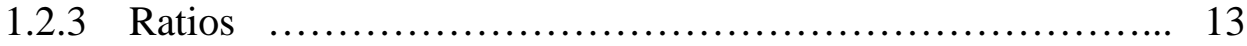

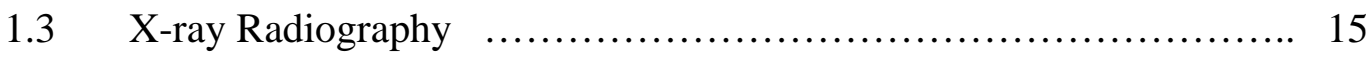

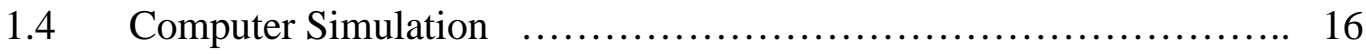

1.4.1 Finite Element Method ….................................. 17

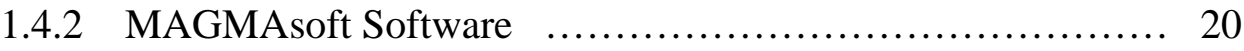

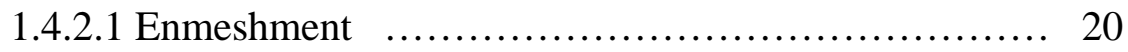

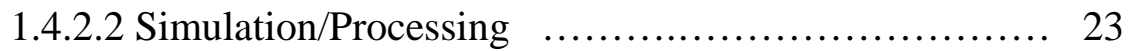

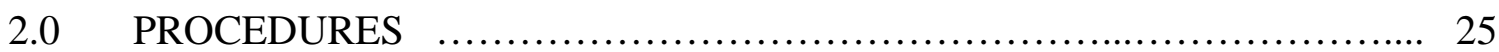


$2.1 \quad$ Materials ............................................... 25

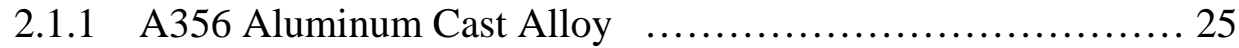

2.1.2 Mold Material ............................................ 26

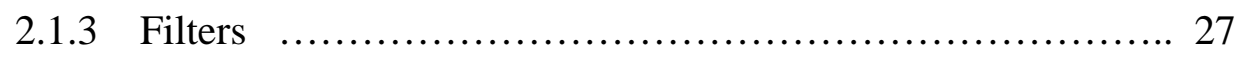

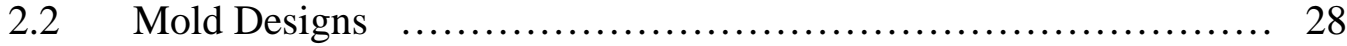

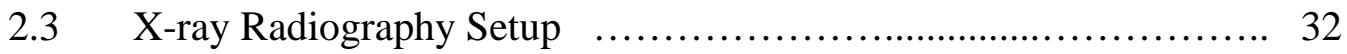

$2.4 \quad$ Process Parameters ............................................................... 35

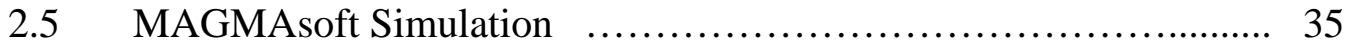

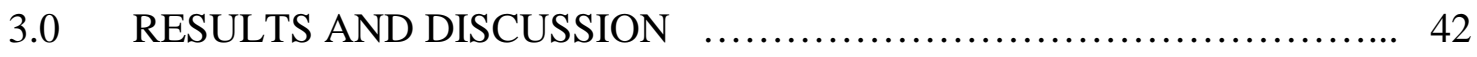

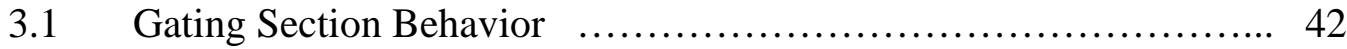

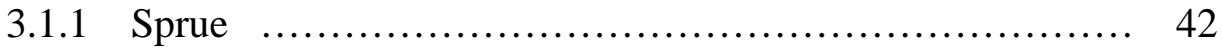

3.1 .2 Sprue-well $\ldots \ldots \ldots \ldots \ldots \ldots \ldots \ldots \ldots \ldots \ldots \ldots \ldots \ldots \ldots \ldots, 44$

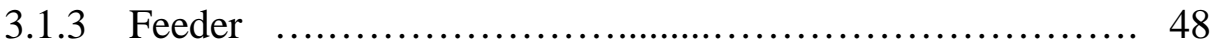

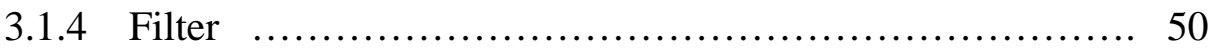

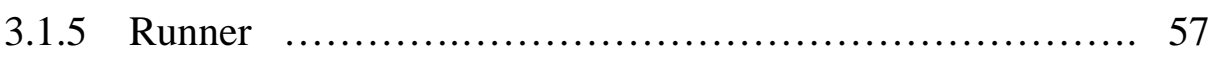

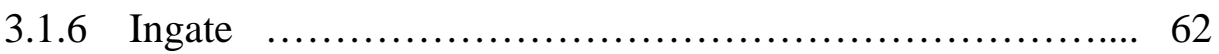

3.2 Gating System Behavior ....................................................... 62

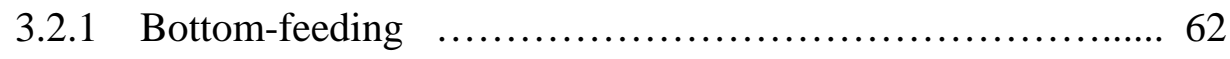

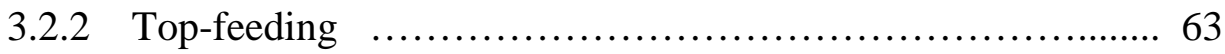

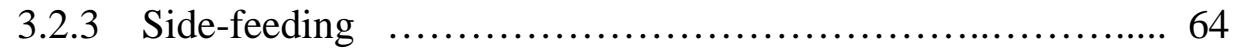

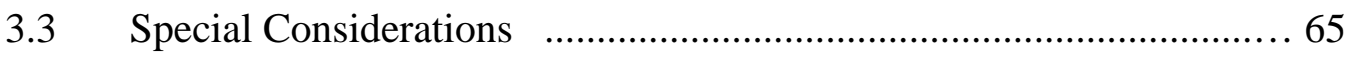

3.4 Evaluation of Mold Designs....................................68

3.5 The Effect of Insulating Coatings.............................. 71

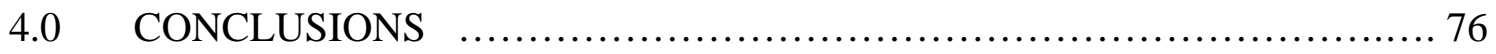

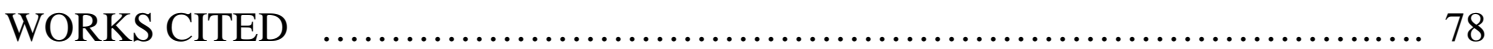

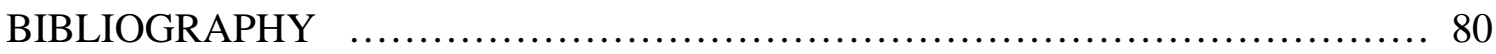




\section{ACKNOWLEDGEMENTS}

This research investigation was supported by the Department of Energy, Office of Industrial Technology through the Cast Metal Coalition program. Guidance for this work was provided by the Permanent Mold Committee of the American Foundry Society. The efforts of Dr. Joseph Santner, Director of Research at AFS and the members of the committee are gratefully acknowledged.

Arrow Aluminum in Avon Lake, $\mathrm{OH}$ made a substantial in-kind contribution to the project by providing access to a production real-time X-Ray unit, molten aluminum and general support with the experiments.

Some of the graphite molds evaluated in this study were machines as costshare by Hayes Lemmerz. The technical support and advise from Mr. Chad Bullock and Bernie Jaeger of Hayes Lemmerz is gratefully acknowledged. Assistance with filters and expert advise on filters and filter print design were received from Dr. Philip Sandford at Foseco. These contributions are gratefully acknowledged.

This publication was prepared with the support of the U.S. Department of Energy (DOE), Award No. DE-FC07-01ID13983. However, any opinions, findings, conclusions or recommendations expressed herein are those of the authors and do not necessarily reflect the views of the DOE. 


\section{ABSTRACT}

This report summarizes a two-year project, DE-FC07-01ID13983 that concerns the gating of aluminum castings in permanent molds. The main goal of the project is to improve the quality of aluminum castings produced in permanent molds. The approach taken was determine how the vertical type gating systems used for permanent mold castings can be designed to fill the mold cavity with a minimum of damage to the quality of the resulting casting. It is evident that somewhat different systems are preferred for different shapes and sizes of aluminum castings. The main problems caused by improper gating are entrained aluminum oxide films and entrapped gas. The project highlights the characteristic features of gating systems used in permanent mold aluminum foundries and recommends gating procedures designed to avoid common defects. The study also provides direct evidence on the filling pattern and heat flow behavior in permanent mold castings.

Equipment and procedure for real-time X-Ray radiography of molten aluminum flow into permanent molds have been developed. Other studies have been conducted using water flow and the behavior of liquid aluminum in sand mold using real time photography. This investigation utilizes graphite molds transparent to X-Rays making it is possible to observe the flow pattern through a number of vertically oriented gating systems. These have included systems that are choked at the base of a rounded vertical sprue and vertical gating systems with a variety of different ingates into the bottom of a mold cavity. These systems have also been changed to include gating systems with 
vertical and horizontal gate configurations.

Several conclusions can be derived from this study. A sprue-well, as designed in these experiments, does not eliminate the vena contracta. Because of the swirling at the spruebase, the circulating metal begins to push the entering metal stream toward the open runner mitigating the intended effect of the sprue-well. Improved designs of sprue-wells should be evaluated. In order for a runner extension to operate efficiently, it must have a small, squared cross-section. If it is tapered, the first metal to enter the system is not effectively trapped. If the cross-section is large, there is less turbulence when aluminum enters the mold cavity in comparison to the smaller cross-sectioned, squared runner. However, a large runner reduces yield. In bottom-feeding gating systems, a filter can significantly improve the filling of the casting. The filter helps to slow the metal flow rate enough to reduce jetting into the mold cavity. In top-feeding gating systems, a filter can initially slow the metal flow rate, but because the metal drops after passing the filter, higher velocities are achieved during free-fall when a filter is in place. Side-feeding gating systems provide less turbulent flow into the mold cavity. The flow is comparable to a bottom-feeding gating system with a filter. Using a properly designed side-gating system instead of a bottom-feeding system with a filter can potentially save the cost of the filter.

Rough coatings promote better fill than smooth coatings. This conclusion seems at first counter-intuitive. One tends to assume a rough coating creates more friction resistance to the flow of molten metal. In actuality the molten aluminum stream flows inside an oxide film envelope. When this film rests on top of the ridges of a rough coating the microscopical air pockets between the coating and the oxide film provide more thermal insulation than in a smooth coating. This insulation promotes longer feeding distances in the mold as demonstrated in the experiments.

Much of this work is applicable to vertically parted sand molds as well, although the heat transfer conditions do vary from a metal mold generally used in permanent molding of aluminum. The flow measurements were conducted using graphite molds and 
real time X-ray radiography recorded at a rate of 30 images per second through those molds. The facilities at Arrow Aluminum Foundry were used in the study. The results will be employed to demonstrate to the American Foundry Industry how molten aluminum alloys flows in permanent molds of different designs and characteristics. The results of these experiments were compared with computer flow and simulation models. The Procast and Magmasoft flow and solidification simulation programs were employed to predict the flow behavior under the different conditions that can prevail in permanent mold gating. The development of a valid computer model that can correctly and accurately predict this flow is much more intricate than is generally realized. To provide accurate predictions such programs require significant adjustments and verification with experimental data.

The project was conducted at CWRU under the direction of the Aluminum Permanent Mold Gating committee (Phil Sandford of FOSECO, Chairman). Most of the graphite molds were machined at Hayes-Lemmerz Premium Casting plant in Bristol, Indiana; a few were fabricated at other machine shops. The results of the real time X-ray were evaluated jointly and the effect of the variables studied by both CWRU and the AFS Monitoring Committee. The AFS Aluminum Permanent Mold Gating Committee includes numerous technical personnel from various permanent molding foundries. The prior experience that all of these personnel have had in permanent molding and gating techniques has been a valuable resource to this project. 


\subsection{INTRODUCTION}

\subsection{Permanent Vertical Mold Aluminum Casting}

Vertical permanent mold gravity casting of aluminum alloys is cost effective production method of castings, especially in high volume applications. In this method, a metal mold, typically cast iron or steel, is machined with a vertical parting line. The resulting halves are as close to symmetric as possible in order to facilitate even and uniform cooling rates throughout the casting. The metal molding material causes aluminum to solidify quicker than in sand molds, leading to quicker solidification times and smaller secondary dendrite arm spacing. The smaller spacing provides better mechanical properties for the aluminum casting. Other benefits of permanent molds in comparison to sand castings include closer dimensional tolerances, good surface finish resulting from the machined inner surface and lower cost of production because the metal dies can last a long period of time.

Vertical permanent molds and sand molds have many similarities but also demonstrate inherent differences. The basic gating components are the same in both. Just as in sand molds, if vertical permanent molds are filled too quickly, turbulence, oxide formation and trapped air may result. This is important when designing vertical permanent molds because the vertical orientation causes longer distances from the pouring basin to the mold base and results in higher metal velocities. Unlike sand molds, risks of premature solidification and cold shuts develop if the mold is not filled quickly 
enough. Therefore, gating must be designed to allow for proper flow rates in both mold types, but especially in vertical permanent molds [1, 2].

\subsection{Accepted Industry Practices}

In recent years, the aluminum foundry industry has been using a general set of practices for gating permanent mold aluminum castings. These principles were developed using historical "lessons learned" in an evolutionary process [3-5]. Few of these principles have been either confirmed or refuted through experimentation and simulation. Some studies of general gating, not applied specifically to the permanent mold process, have been done by John Campbell [6, 7] and the Battelle Research Laboratories.

Because of this lack of experimentation, few improvements have been made to the current gating practices. Furthermore, many gating systems are developed using these principles with little understanding as to what happens in the mold during filling. A lack of true gating understanding causes many foundries to produce poor quality castings. Molds that work in one foundry may not work in another one because of process inconsistencies. Defects may arise in the casting that are direct results of poor gating practices implemented by blindly following gating guidelines. By gaining a better

understanding of these methods and practices, improvements in casting yields and integrity should be realized.

There are several objectives to efficient gating design. The first is to prevent 
excess turbulence. When the metal flows in a very turbulent manner, excessive liquid metal surface area is exposed to air. This maximizes the opportunities for oxide formation. Furthermore, as the oxide mixes with the metal, the oxide films and particles become entrapped within the alloy. These trapped oxide inclusions form regions of high, localized stress when a load is placed on the cast part [8]. Another reason to avoid excess turbulence is to prevent air entrapment that leads to porosity in the casting. Just as bubbles form in water when moved in a violent manner, air bubbles form in metal when the flow is very turbulent. Because of the rapid solidification rate in permanent molds, bubbles may be trapped in the final, solidified casting. At the same time the gating system design has to ensure the flow rate is not too slow. The heat sink behavior of metal permanent molds makes castings susceptible to chilling and premature solidification. Premature solidification can produce cold shuts and other casting defects [9]. Examples of these defects are shown in Figure 1. To avoid this situation, metal flow must be constant and fast enough to prevent thin sections from freezing.

\subsubsection{Gating Components}

Vertical permanent mold gating systems have several basic parts. These include a sprue, sprue-well, riser, runner, ingate and, sometimes, a filter. Gating systems are formed with various combinations of these six sections. Components and placement in the system are determined by the final casting dimensions, the type of metal used and the type of foundry in which the parts will be produced. Figure 2 shows each of these gating components in a schematic. 


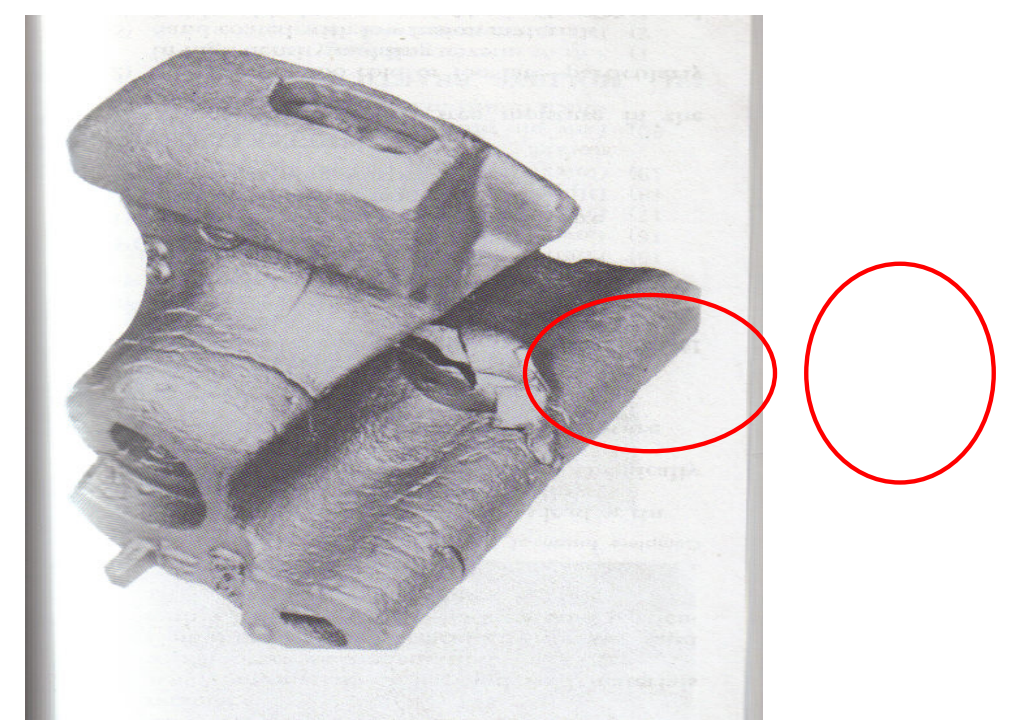

Figure 1: Examples of cold shut casting defect [9].

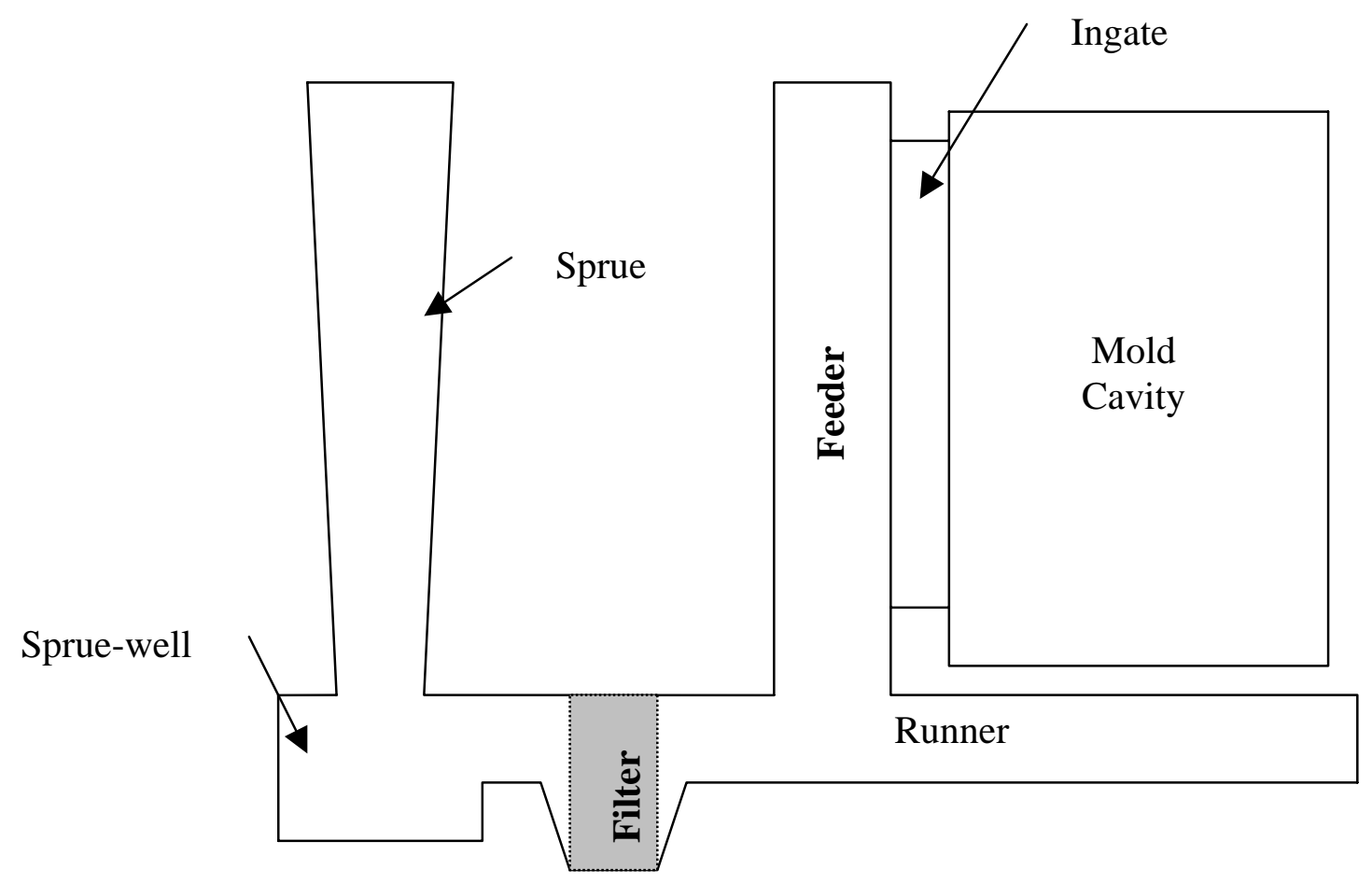

Figure 2: Schematic of mold components. 


\subsubsection{Sprue}

In a vertical permanent mold, the metal enters into the mold via a sprue. Sometimes, above the sprue, the molten aluminum is initially poured into a pouring basin. In our experiments, an insulated pouring basing was used. The height of the metal exerts a "head pressure" on the system. The pressure is calculated as:

$P=\rho g h$

(Equation 1)

where $\mathrm{P}$ is the pressure at the bottom of the basin, $\rho$ is the density of the aluminum, $\mathrm{g}$ is the acceleration from gravity $\left(9.8 \mathrm{~m} / \mathrm{s}^{2}\right)$ and $\mathrm{h}$ is the height of metal in the basin. This pressure is the driving force to feed the mold. As metal travels down the sprue, it accelerates from gravity. Because of this increase in velocity, this is the area of the mold where the most turbulence is expected to occur. This turbulence allows large surface areas of metal to come in contact with the reactive ambient air and form oxides.

Typically, the sprue is tapered to be narrower at the base. The taper has several functions, including preventing aspiration and entrapment of air from entering the molten metal stream. As previously mentioned, relatively quick solidification rates in permanent mold systems cause air to become trapped in the casting and form porosity. It has been acknowledged that a minimum taper of 0.008 centimeter per one centimeter of sprue length should be used [8]. The sprue can also be used to facilitate a fixed rate of metal flow based on the height and diameter. Through proper design, the operator can achieve repeatable fill times with minimum variation by keeping the sprue full until the mold is filled. Sprue design is one of the most important components of gating $[10,11]$. 


\subsubsection{Sprue-well}

As pointed out by Campbell, a sprue-well can be beneficial, but not absolutely necessary to a casting's integrity [8]. When the metal has completed its controlled flow down the sprue, it moves at rather high velocities. Then, to enter the runner, it has to change direction and flow at a right angle relative to the sprue. This causes excessive amounts of splashing in the runner system. Again, this exposes large amounts of metal droplets to atmospheric conditions. These smaller particles can begin to freeze sooner than in a laminar flow of metal [12]. By placing a small basin or "well” at the bottom of the sprue, the molten metal can hit the bottom and lose excessive kinetic energy before entering the remaining portions of the gating system. This allows for a reduced turbulent flow of metal into the casting and helps to prevent or reduce the effects of the vena contracta shown in Figure 3. The vena contracta causes the metal to actually suck in air. This phenomenon was confirmed by P. Webster and can actually be heard in a quiet foundry [13].

Generally, the height of the well should be twice that of the runner, and the width/diameter should be roughly double that of the sprue base. The overall size of the sprue well may be increased for larger sprue heights to accommodate for the larger amount of kinetic energy produced from the larger drop. In order to avoid swirling of the metal, the diameter of the sprue is typically non-circular in shape. 


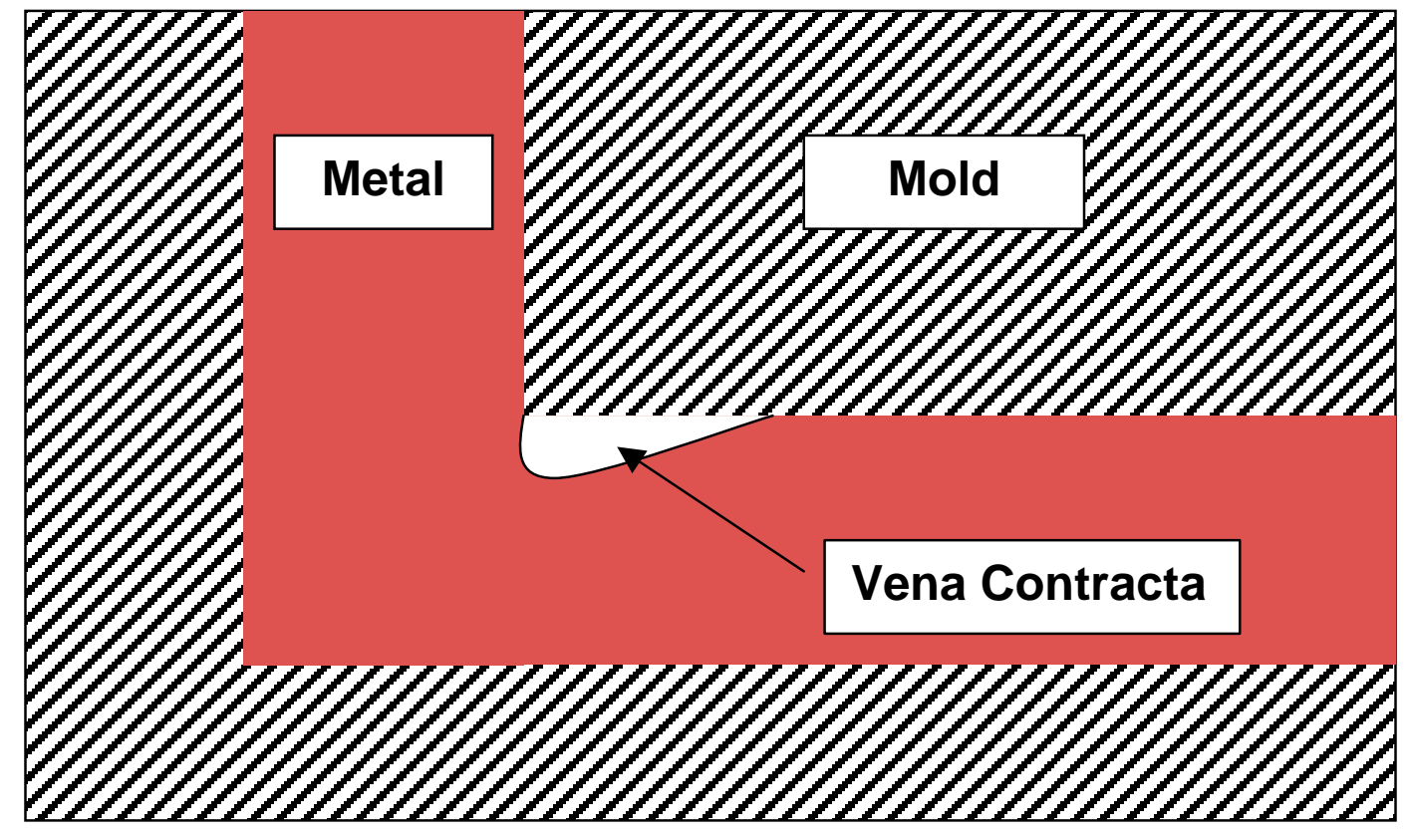

Figure 3: Schematic of vena contracta. 


\subsubsection{Feeder}

The feeder of a gating system is used to provide metal to the casting during solidification. As the metal begins to freeze in the mold cavity, it begins to shrink. Depending on the geometry of the casting, shrinkage can form. Therefore, it is vital to provide a constant supply of hot metal to the system to fill in the voids left by shrinkage. The feeder is used to provide this hot metal. For this experimentation, the only mold utilizing a feeder is Mold 4, which is a web-gated system. The hottest metal in the system is stored in the feeder and supplied to the casting, via the web gate, as needed. In most cases, the height of the feeder must be slightly higher than that of the actual casting. This allows for a small amount of excess pressure to counteract capillary forces. Since this excess pressure is needed and the feeder size cannot be extreme and reduce yield, the actual dimensions of feeders in a web gating system require careful calculation. 


\subsubsection{Filter}

Filters have two main purposes in a gating system. They are inserted to help remove slag and dross from the metal as it enters the mold cavity. They are therefore usually placed close to the mold cavity entrance. The efficiency of filters increases with a large surface area and a decrease in metal velocity through the filter. The filter porosity adds the inherent benefit of reducing metal flow and decreasing turbulence in the gating as metal moves through the pore system. When the metal exits the filter, the metal flow tends to be less turbulent.

Several ways exist in which filters extract slag and dross, leaving behind clean metal. The first way is to prevent larger portions of slag from even entering the filter. This is controlled by the amount and size of the pores within the filter. Besides the porosity of the filter, several other factors determine how well a filter will remove slag. The surface texture, geometric shape and the range of microporosity in the internal passages have a significant bearing on filtering efficiency. For instance, two identical porosity filters can have significant differences in filtering ability based upon these factors [14]. 


\subsubsection{Runner}

The runner of the gating system is generally placed before the ingate. When the metal reaches the runner, it has already passed through the majority of gating components in place to reduce turbulence and slag. Therefore, it is extremely important that the runner will reduce turbulence or the opportunity for the metal to trap air. Typically, the runner is designed with large radii at the corners and angles greater than $90^{\circ}$ in order to provide a gradual change in flow path. This helps prevent the surface oxide skin from breaking and exposing more metal to the reactive atmosphere. Many gating systems have extensions in the runner sections after the ingate. These extensions are used to trap the first metal to enter the casting, since it may contain the most oxide. These extensions help to decelerate the metal prior to entering the mold cavity. In nearly all gating systems, the runner is placed below the ingate, providing the metal flow a means of slowing down as it moves along the runner prior to entering the mold cavity. The runner extension also allows the metal to reduce its velocity, since the volume of the runner is increased. This reduces the turbulence as the metal enters the mold cavity [15]. 


\subsubsection{Ingate}

Metal flow conditions at the ingate are important to the entire gating system. A slow rate of flow is desired at this location since this is the area where the metal enters the mold cavity. The flow conditions dictate the integrity of the final casting. The ingate can be placed at several locations in the mold, depending on casting geometry and the gating system type [16].

\subsubsection{Gating Systems}

\subsubsection{Top-feeding}

In top-feeding, the entire gating system, including ingate, is placed above the mold cavity. Upon entering the mold, the metal falls from the ingate to the bottom of the casting. This can be undesirable, as a large amount of turbulence can result and the higher velocities attained in the mold cavity can erode the mold surface after several uses. In the gating, this may not be much of an issue, but in the casting itself, surface finish is oftentimes critical. One advantage to this system is that not much gating is needed and therefore a higher yield is realized if a casting of high integrity is produced. 


\subsubsection{Bottom-feeding}

In bottom-feeding systems, metal enters the mold cavity from the bottom of the casting, but slows the filling process somewhat and may require a higher pouring temperature. When properly designed, the metal gradually enters the mold cavity without jetting, aiding in producing a sound casting. However, longer solidification time results from the slower filling process.

\subsubsection{Side-feeding}

The side-feeding method, commonly referred to as web gating, utilizes a large riser positioned on the side of the casting. This is a gating system where the riser is connected to the entire height of the casting via a thin ingate section. This design locates the hottest metal in the top of the riser called a feeder at this location. Forces from the thin ingate provide sufficient resistance to the metal flow to slow the flow rate. This provides less turbulence of the metal entering the mold cavity. The reduction in flow rate has the potential to eliminate filtering as a flow rate reducer. Several disadvantages, however, occur when using side-feeding. For instance, because of the location of the ingate, a large amount of machining is often necessary to achieve the desired shape. No reliable method for calculating the width and thickness of the ingate exists. 


\subsubsection{Ratios}

Many authors of previous gating studies have determined the ratios for gating designs as a useful tool. These ratios are made to determine the cross sections between several portions of the gating system. The standard convention takes the ratio of the sprue choke, runner and ingate, where the sprue choke is the smallest cross sectional area in the sprue. Examples of where these measurements are taken can be found in Figure 4. Most permanent molds for aluminum follow the standard system, often referred to as ratios of enlargement. This standard system is accepted to be 1:2:5. The reason for this progressive increase in cross sectional area is to reduce the metal flow rate. This results in a less turbulent flow in the metal. Generally, the cross section of the runner should be at least double that of the sprue choke. In cases of extraordinarily long sprue lengths, the runner area can increase to nearly four times that of the sprue choke [8]. 


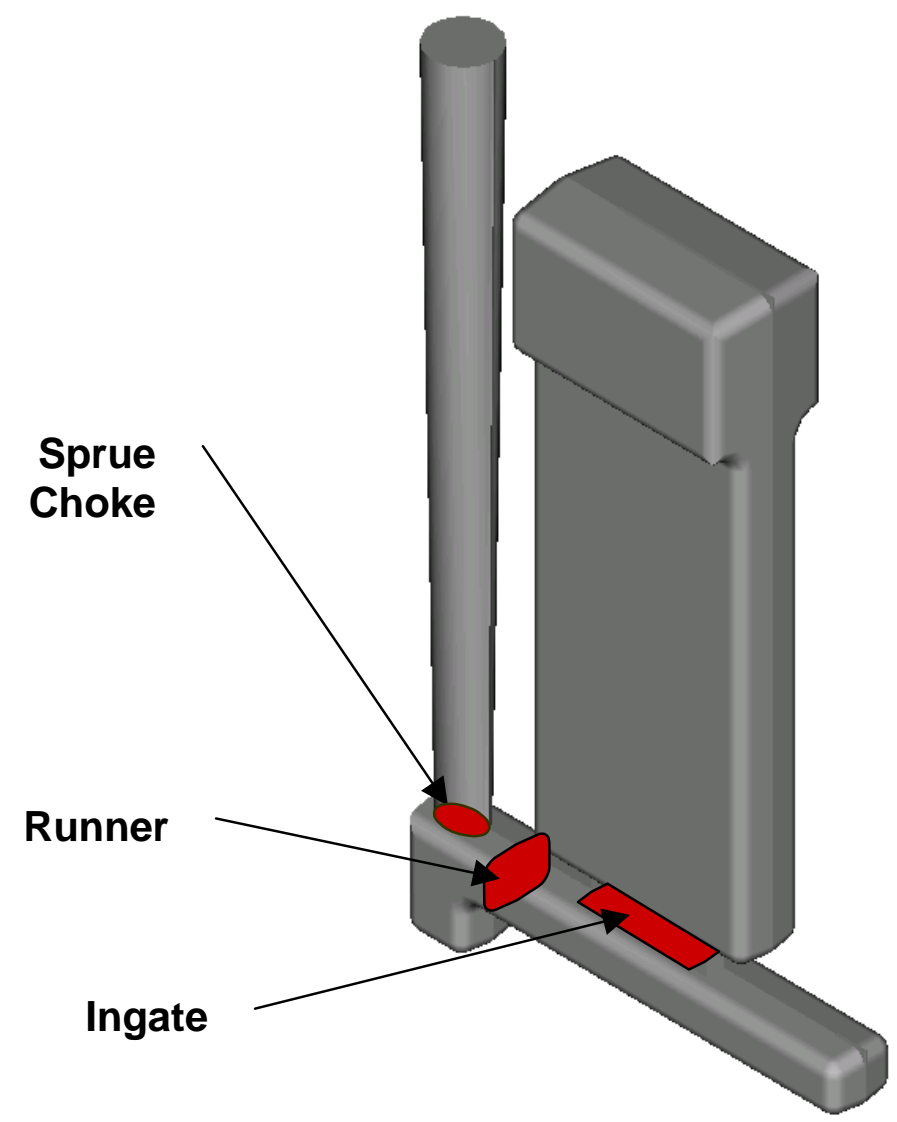

Figure 4: Schematic example of the ratio measurement locations. 


\subsection{X-ray Radiography}

X-rays were discovered in 1895, and records of "industrial” radiographs were taken as early as 1896 [17]. By emitting X-rays from a source, they can pass through opaque objects and produce images when they come in contact with a film to produce an image of the flow behavior's inner workings. In this study, a $160 \mathrm{kV}$ X-ray source was utilized. At this voltage, up to 3 inches of aluminum, but only about 0.5 inches of $\mathrm{H}-13$ tool steel, can be penetrated. Unlike steel, graphite is more transparent to X-rays while also being readily machinable and possessing similar thermal properties. Graphite can be therefore as a substitute for standard tool steel molds in real-time X-ray studies. After the X-rays pass through the mold, they pass through thin, dense intensifier screens. The intensifier screen allows for shorter exposure times in radiography, therefore allowing for real-time filming of the filling process. Once passing through the intensifier screen, the X-ray image is recorded by a Charged Couple Device (CCD) camera. The signal is then passed on to a computer and recorded [18-20]. 


\subsection{Computer Simulation}

As computers have become more powerful and achieved the ability to produce several iterations of solving equations in small amounts of time, developers have created software to produce simulations of physical processes. By using equations to model the behavior of fluid flow processes, predictions of casting behavior can be achieved. These predictions allow for improved shapes of prototype dies. Simulations can also be used to understand flow patterns in a mold and to resolve casting defects caused by filling [21, 22].

Of primary importance in flow simulation is the geometry of the mold and casting. Once defined, computer programs use databases of information and can permit the mathematic calculation of how the combination of geometry, materials and process parameters will interact. The results from these calculations are then displayed in the form of graphs and charts, movie sequences, and/or three-dimensional representations of the casting. These visualization techniques allow to determine how to gate a casting properly. The necessary changes can be made to the mold geometry in the threedimensional computer model and then run the simulation software again. This method is much cheaper than machining changes into a mold or pattern. 


\subsubsection{Finite Element Method}

Finite Element Method (FEM) is a type of numerical method for solving complex equations. The underlying principle behind FEM is to divide a large object into several smaller, more manageable pieces. In the case of applying fluid flow equations to a large geometric piece, the geometric sample is divided into several smaller cells or elements using a meshing process. These smaller cells are typically simple shapes like triangles or squares. Figure 5 shows that as the size of these simple shapes decrease, they become closer to approximating the overall geometric shape. The intersections of the lines making up these cells are known as nodes or elements. These elements are represented in a three-dimensional matrix since they represent a three-dimensional object. Basic functions are created for each of these points and then combined linearly to create an approximate solution to the given problem [23].

In the case of casting simulations, FEM is applied to variations of the basic fluid flow equation of Navier-Stokes. For these equations, the assumptions that the fluid is incompressible and of Newtonian character are made. Terms have been added to the equations to account for temperature-dependent viscosity of the metal and non-linear turbulent viscosity. Since the Navier-Stokes equation is solved with a two-equation model, the solution becomes more complicated. In the case of three-dimensional modeling, the solution requires six coupled non-linear equations [24]. 


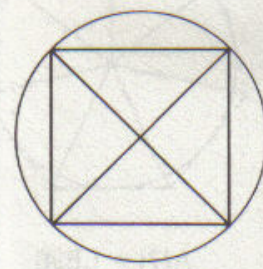

$A(4)=1.125$ units
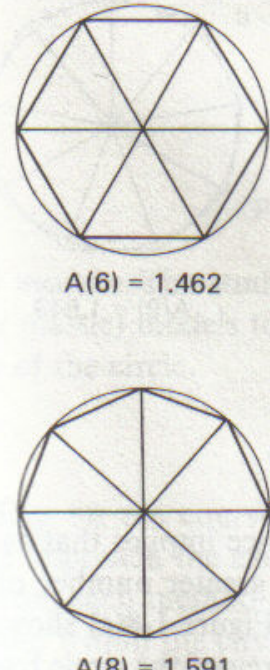

$A(8)=1.591$
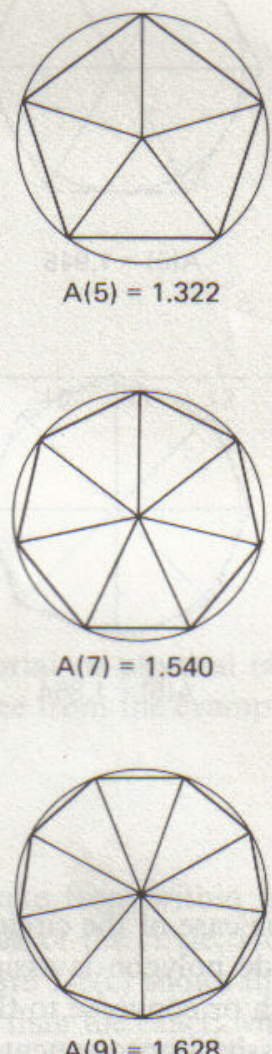

Figure 5: Schematic showing that increasing cell numbers result in a better geometrical fit. [25] 
Solutions are found using the following algorithm developed by Taylor [26]:

1) Specify boundary conditions for pressure, velocity, turbulent kinetic energy and dissipation

2) Estimate initial values for velocity, pressure, turbulent kinetic energy and dissipation

3) Use values of kinetic energy and dissipation to determine the point-wise distribution of turbulent viscosity

4) Evaluate the distribution of the four values

5) Return to step 3 until convergence has been achieved.

Several iterations of this algorithm are performed for each node in the elemental mesh. It is necessary to have ample amounts of computing power in order to complete these iterations in a reasonable amount of time. 


\subsubsection{MAGMAsoft Software}

Developed in Germany in the late eighties, MAGMAsoft is widely accepted as the standard computer modeling package for metal flow and solidification simulation. Utilizing the Finite Element Method (FEM) of numerical analysis, previous studies have shown MAGMAsoft to be relatively close to actual casting results [27]. Included in MAGMAsoft's standard package is the ability to produce both flow and solidification simulations. The flow module, MAGMAflow, contains the ability to both see the temperature of metal at various points in time during the filling process and to follow a point in the metal throughout the flow process by placing electronic tracer particles into the melt.

\subsubsection{Enmeshment}

To perform the mathematical calculations necessary for a three-dimensional simulation, a geometric model of the casting must be divided into several smaller objects known as cells. These objects must be simple shapes, such as triangles, tetrahedrons, prisms, etc. This process is called meshing or enmeshment. A sample of an unmeshed and a meshed model can be seen in Figures 6 and 7, respectively. The mathematical calculations for fluid flow are then performed on these meshed subregions. The complexity of the part geometry dictates the number of elements or cells used in a simulation. A dense mesh of very small cells typically provides for a more accurate 
simulation, but requires a very long simulation time. The cell size is also dictated by the desired accuracy of the geometric representation. So, as accuracy of the simulation and geometric representation increase, the time to run the simulation also increases.

MAGMAsoft contains a module that produces automatic enmeshment. This module takes the geometric form and automatically breaks the shape down into smaller, more manageable rectangular cells. The user inputs the desired number of elements, or points where lines of a cell cross. The automatic meshing algorithm then determines a compromise between accuracy and simulation time using the defined number of elements. 


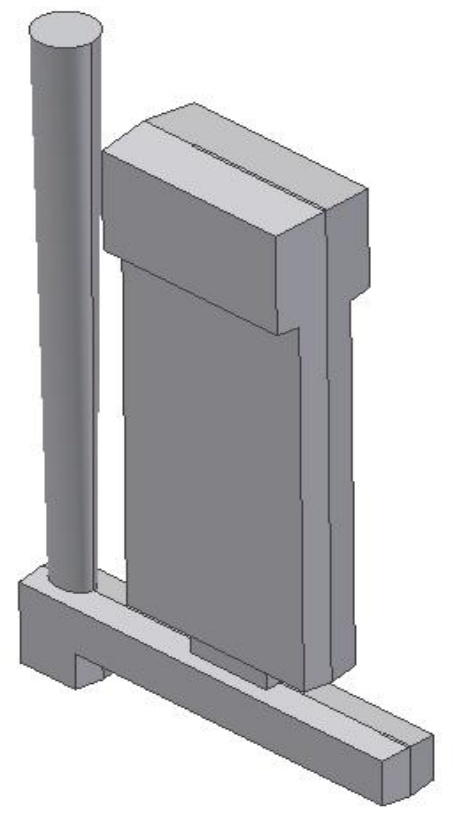

Figure 6: Sample of solid CAD model.
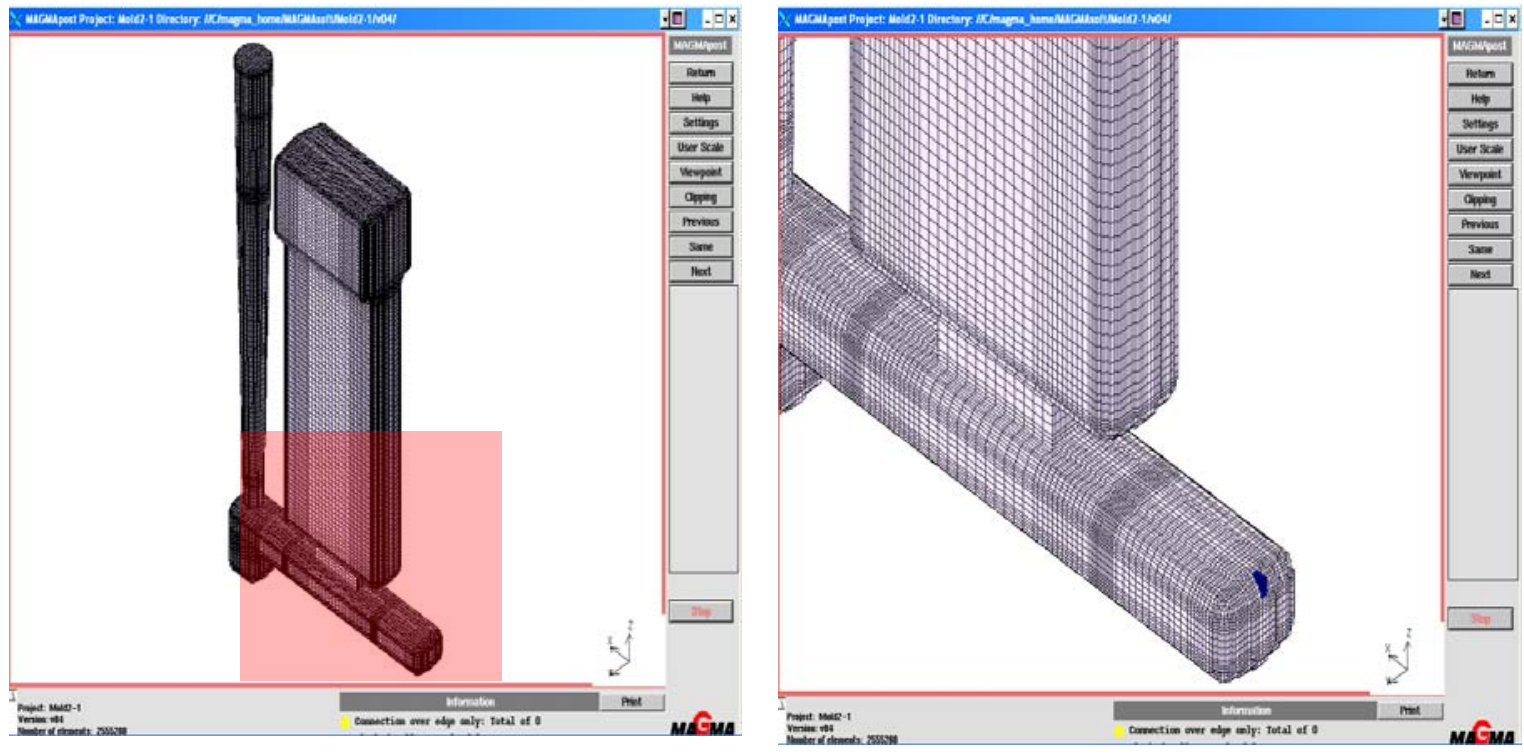

(a)

(b)

Figure 7: (a) Large sample of meshed geometry. (b) Zoomed sample of meshed geometry. 


\subsubsection{Simulation/Processing}

Once the geometry is meshed, MAGMAsoft uses a modified Navier-Stokes equation for modeling fluid flow. The user is prompted to first input the materials that make up the mold, the casting, and the filter if one is used. From the material entries, various thermal and physical properties of the metal are extracted from a central database built-in to MAGMA. This data includes melting temperature, heat capacity, viscosity versus temperature, etc. The user then enters the temperature of the various materials at the time of casting. Once all the data is entered into the system, the simulation is started. Simulations can run from hours to days, depending on the complexity of the part geometry and the number of elements/cells in the system. For the simulations performed in this experiment, simulations typically ran for $1-2$ days.

After the simulation is completed, the data generated during the simulation is transferred to a postprocessor. This portion of MAGMAsoft takes the data and manipulates it to show visual representations. For instance, raw data can be displayed as a bulk three-dimensional flow pattern with different colors representing varying temperatures in the melt. An example of this bulk representation can be found in Figure 8. This data can also be used to show intricate patterns in the flow behavior through the use of tracers. A sample tracer plot can be found in Figure 9. 


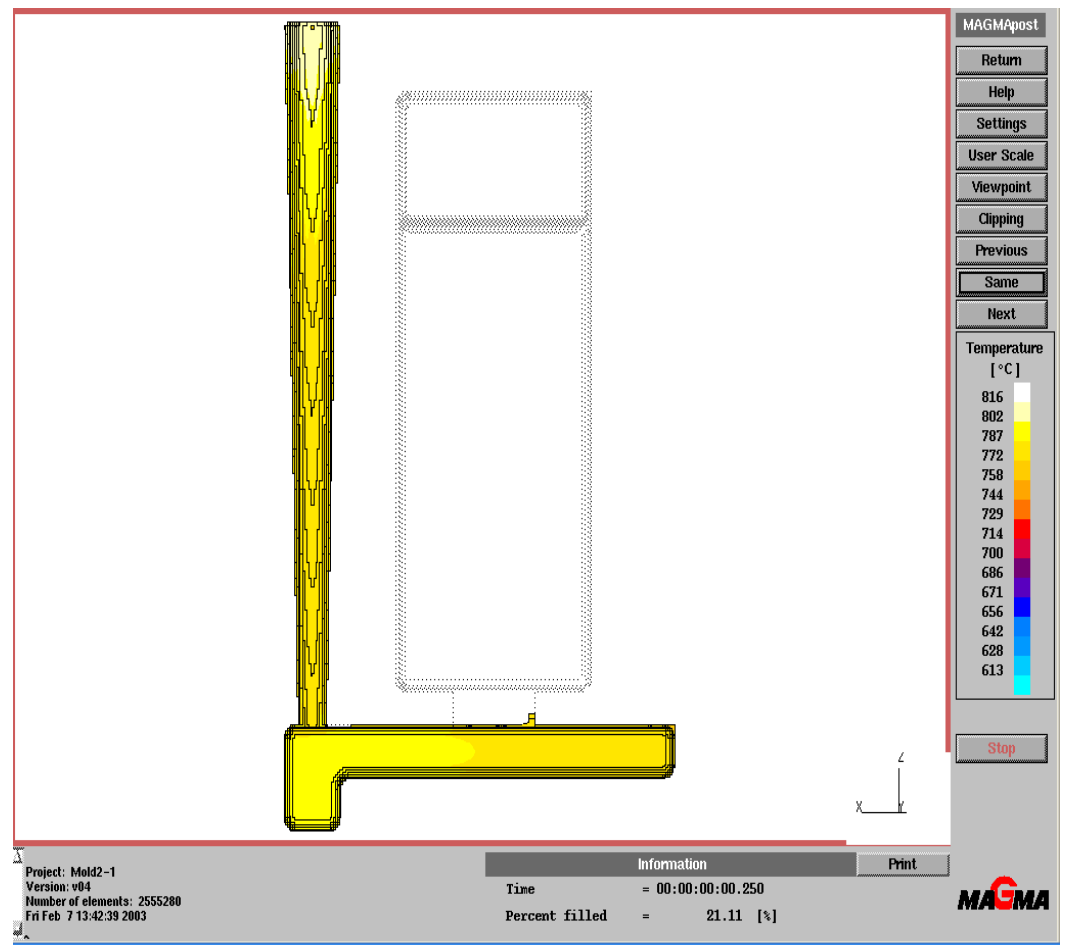

Figure 8: Bulk flow pattern generated by MAGMAsoft.

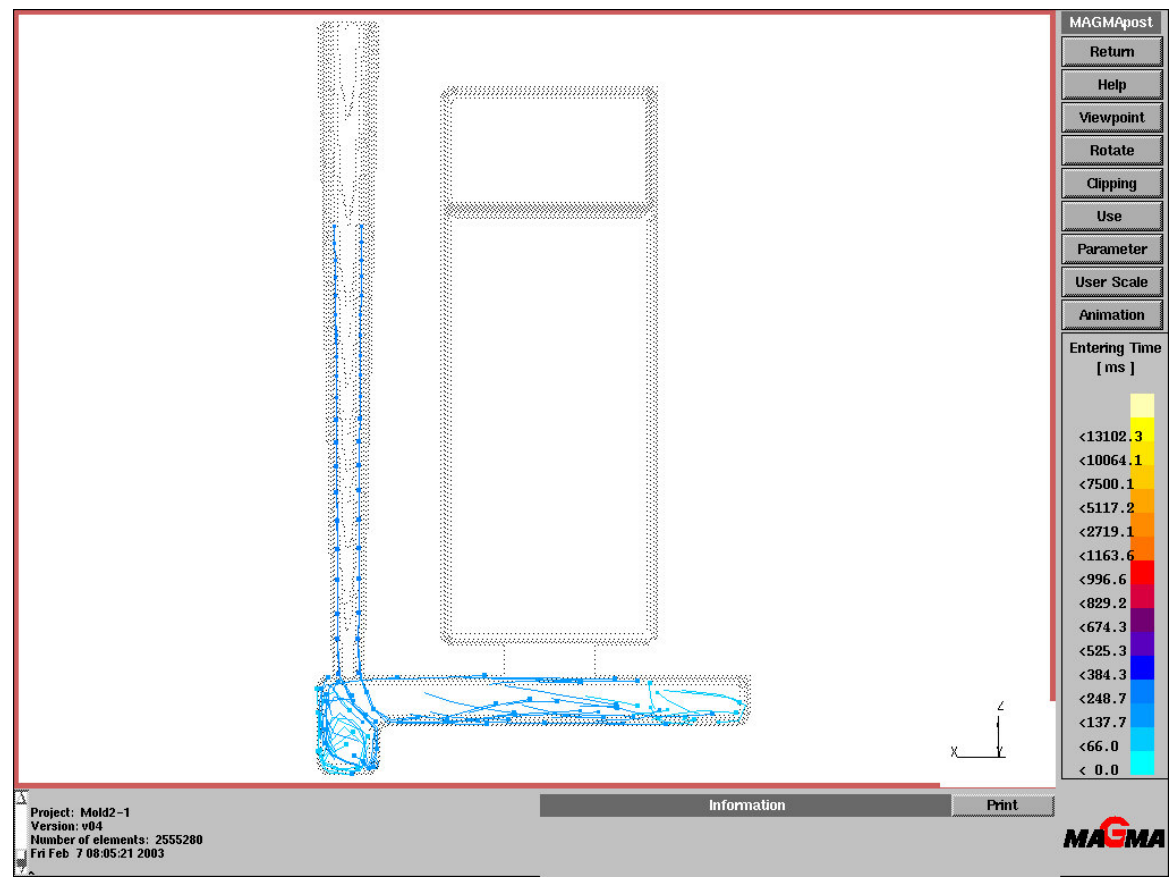

Figure 9: Tracer flow pattern generated by MAGMAsoft. 


\subsection{PROCEDURES}

\subsection{Materials}

\subsubsection{A356 Aluminum Cast Alloy}

A356 is a common aluminum alloy that contains significant amounts of silicon and magnesium as seen in Table 1. The chemistry provides for excellent strength and machinability. The physical, mechanical and thermal properties of A356 are shown in Table 2.

\begin{tabular}{|l|c|}
\hline \multicolumn{1}{|c|}{ Element } & wt. \% \\
\hline Aluminum & 92.05 \\
\hline Silicon & 7.00 \\
\hline Magnesium & 0.35 \\
\hline Manganese & 0.10 \\
\hline Copper & 0.20 \\
\hline Iron & 0.20 \\
\hline Zinc & 0.10 \\
\hline
\end{tabular}

Table 1: A356 aluminum base chemistry. 


\begin{tabular}{|l|r|}
\hline \multicolumn{1}{|c|}{ Property } & \multicolumn{1}{c|}{ Value } \\
\hline Solidus & $542 \mathrm{C}$ \\
\hline Liquidus & $613 \mathrm{C}$ \\
\hline Density & $2460 \mathrm{~kg} / \mathrm{m}^{\wedge} 3$ \\
\hline Latent Heat & $430.518 \mathrm{~kJ} / \mathrm{kg}$ \\
\hline Heat Capacity & $1150 \mathrm{~J} / \mathrm{kgK}$ \\
\hline Viscosity & $4.00 \mathrm{E}-06 \mathrm{~m} \mathrm{~m}^{\wedge} / \mathrm{s}$ \\
\hline
\end{tabular}

Table 2: A356 aluminum properties taken above the melting temperature.

\subsubsection{Mold Material}

Typically, permanent molds for use in aluminum gravity casting are made of either high quality cast iron or tool steel (H13). Since the experiments utilized X-ray radiography, these mold materials could not be used. Instead, the molds were produced with high-strength graphite. Graphite, unlike iron and steel, allows X-rays to easily pass through, so that the aluminum flow can be imaged. The properties of graphite are contained in the MAGMAsoft database and shown in Table 3. Although the thermal properties of graphite are somewhat different from H13 steel, this is not detrimental to this study, since only fluid flow, and not solidification were considered. 


\begin{tabular}{|l|r|}
\hline \multicolumn{1}{|c|}{ Property } & Value \\
\hline Solidus & $1999 \mathrm{C}$ \\
\hline Liquidus & $1999 \mathrm{C}$ \\
\hline Density & $1800 \mathrm{~kg} / \mathrm{m}^{\wedge} 3$ \\
\hline Latent Heat & $0 \mathrm{~kJ} / \mathrm{kg}$ \\
\hline Heat Capacity & $1760 \mathrm{~J} / \mathrm{kgK}$ \\
\hline Yield Stress & $1 \mathrm{MPa}$ \\
\hline Young's Modulus & $5.00 \mathrm{E}+02 \mathrm{Mpa}$ \\
\hline
\end{tabular}

Table 3: Graphite mold material data used for simulations through MAGMAsoft.

\subsubsection{Filters}

Bonded ceramic foam filters were used for this experiment. This type of filter is the largest class of filter used in industry. They are produced by immersing polymeric foam into a ceramic slurry and then sintering. During the sintering process, the polymeric preform vaporizes, leaving only a positive replica of the preform. The filters are made of alumina, mullite, zirconia, silicon carbide and zircon in a wide range of porosities from 10 to 50 parts per inch (ppi) [28]. Actual filter physical and thermal data used in MAGMAsoft cannot be accessed because much of the information is proprietary to the filter manufacturers. Figure 10 shows an example of a 10 ppi ceramic foam filter used in these experiments. 


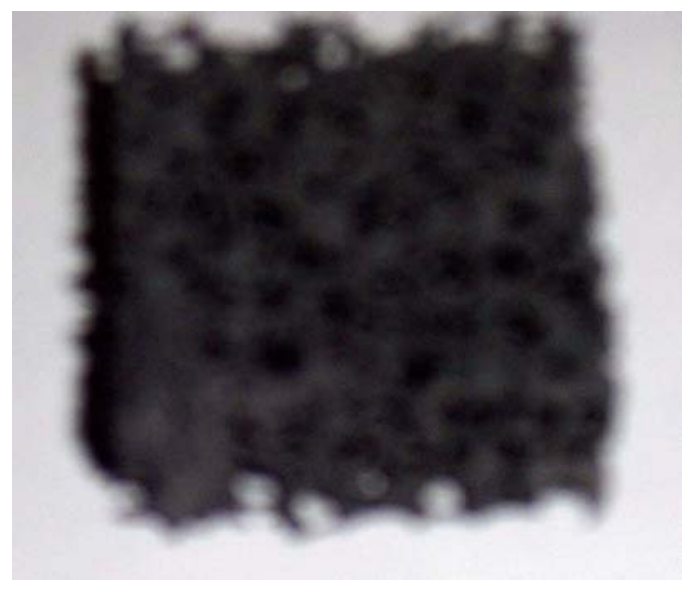

Figure 10: Picture of a ceramic foam filter at $10 \mathrm{ppi}$.

\subsection{Mold Designs}

Five mold designs were utilized in these experiments. These molds were machined in 2000 by Hayes-Lemmerz Precision. Molds 1 - 3, shown in Figures 11 - 16 utilize bottom-feeding gating systems. Mold 1 utilizes a squared runner extension. Mold 2 has a taller, larger cross-section runner and has a tapered end on the runner extension. Mold 3 completely removes the sprue-well and places a filter immediately after the metal hits the bottom of the sprue. Molds 4 and 5, shown in Figures 17-18 and 19-20, respectively, unlike the others, do not use a bottom-feeding system. Mold 4 uses a sidefeeding technique in which a thin ingate feeds the casting from one side. Mold 5 utilizes a top-feeding approach, which is aided by the use of a filter to slow metal before it falls into the mold cavity. All molds that include filters were run one time with a filter and a 
second time without a filter. The molds were designed with specific gating ratios found in Table 4.

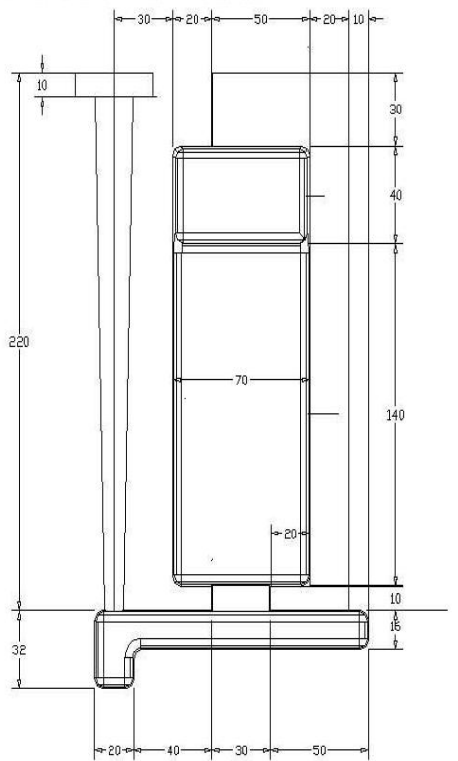

Figure 11: Schematic of Mold 1.

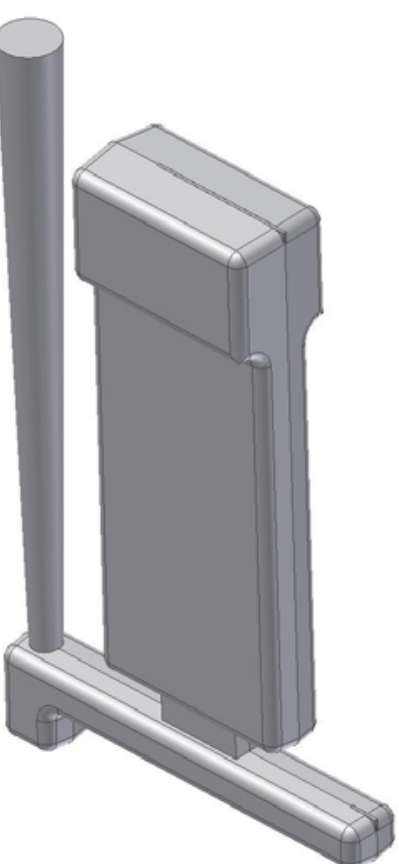

Figure 12: 3-D solid model of Mold 1. 


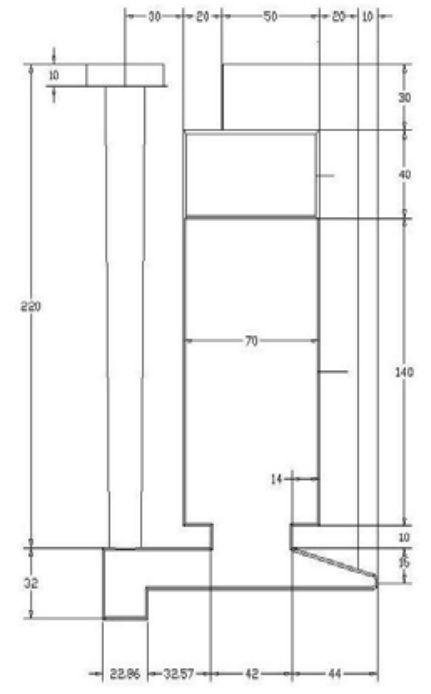

Figure 13: Schematic of Mold 2.

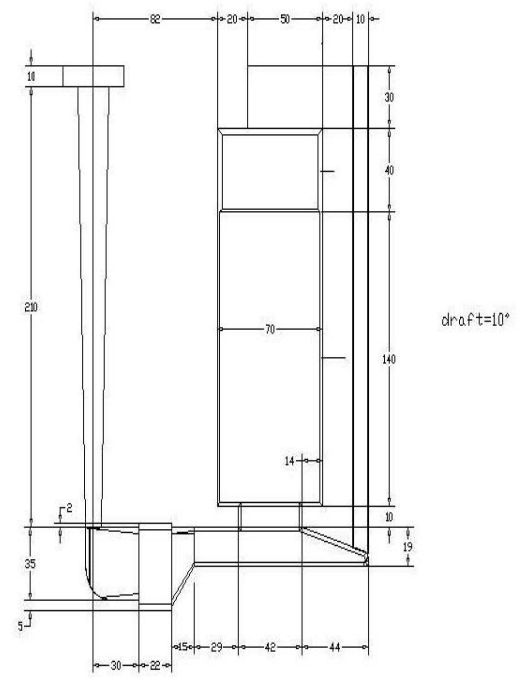

Figure 15: Schematic of Mold 3.

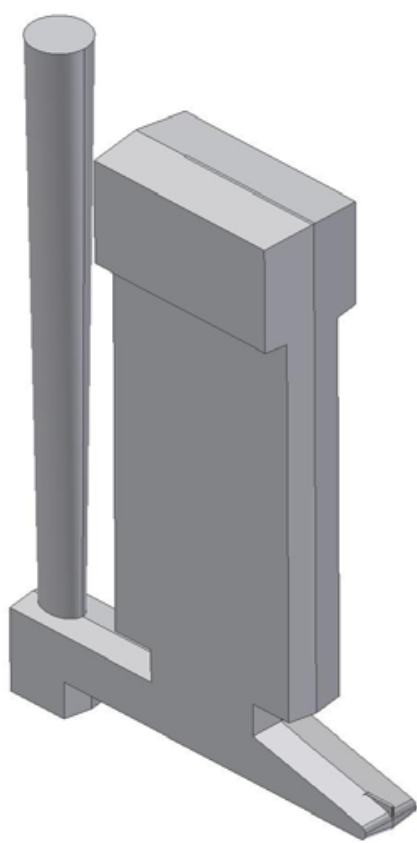

Figure 14: 3-D solid model of Mold 2.

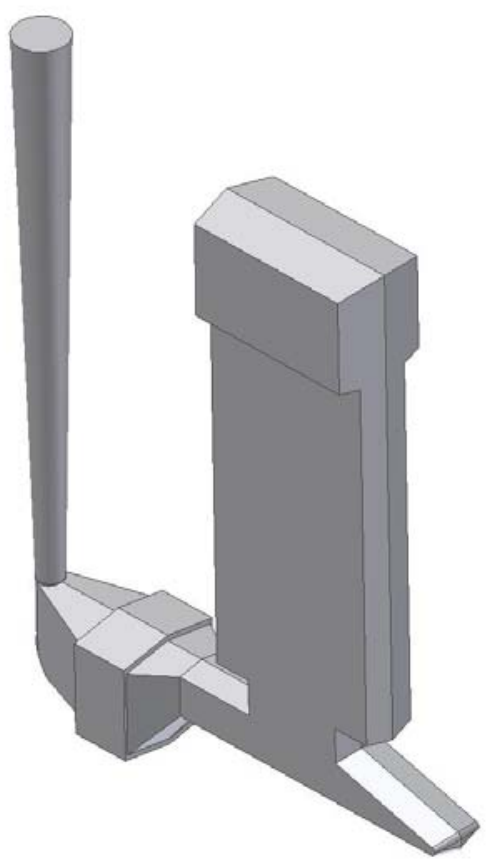

Figure 16: 3-D solid model of Mold 3. 


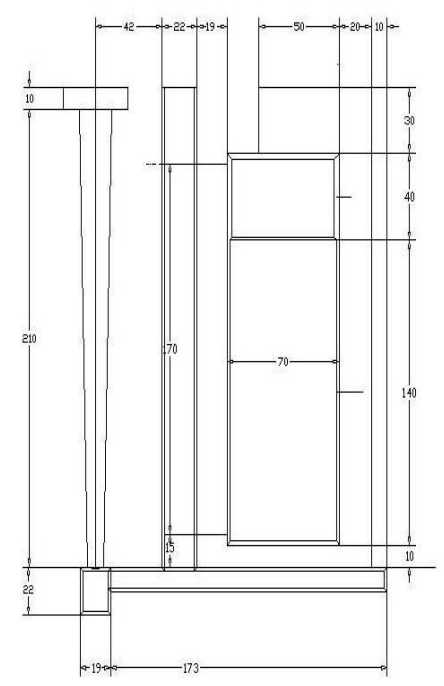

Figure 17: Schematic of Mold 4.

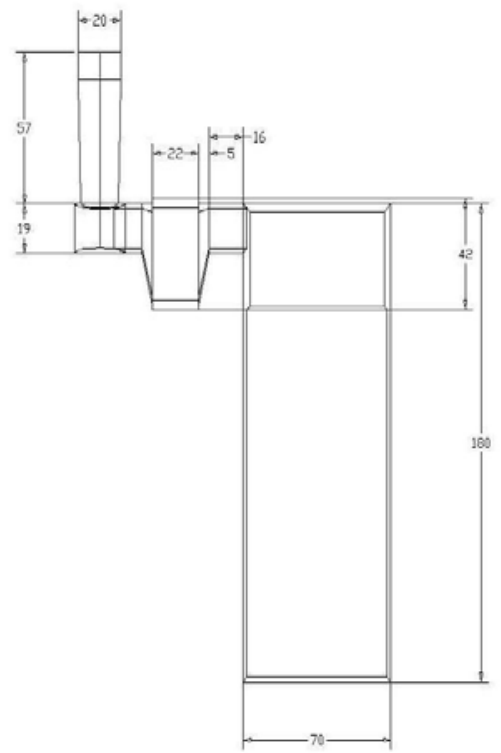

Figure 19: Schematic of Mold 5.

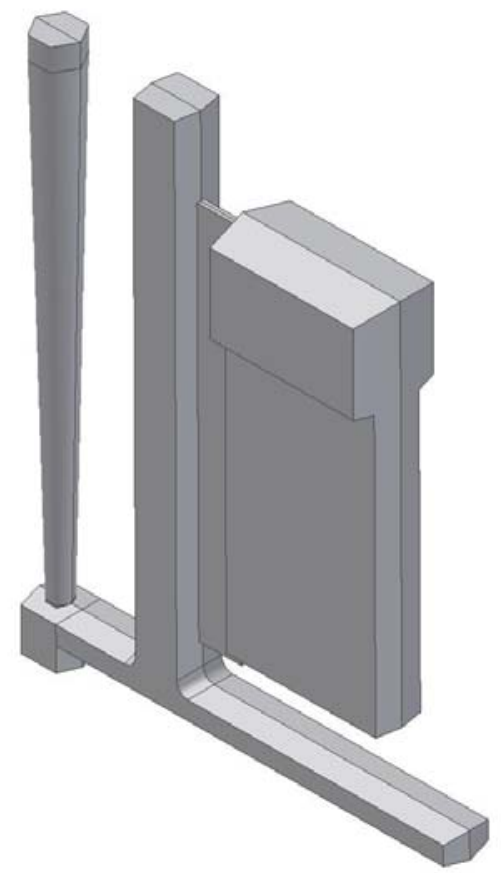

Figure 18: 3-D solid model of Mold 4.

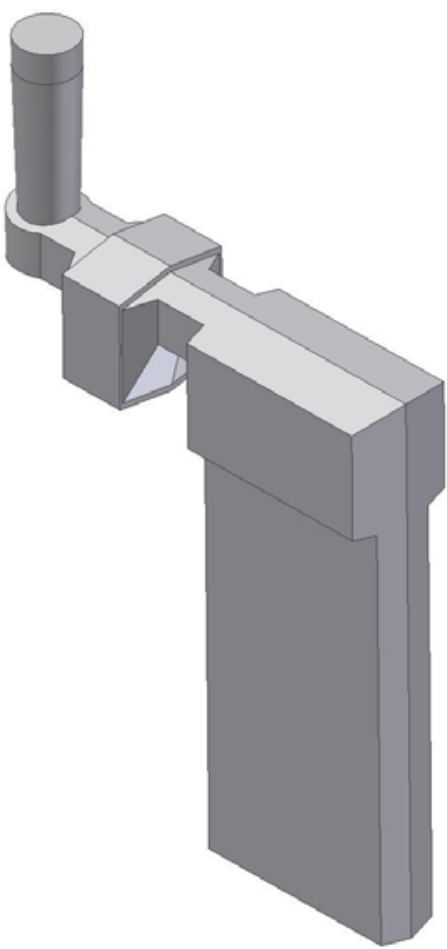

Figure 20: 3-D solid model of Mold 5. 


\begin{tabular}{|c|c|c|c|}
\hline Mold \# & Sprue Choke & Runner & Ingate \\
\hline 1 & 1 & 2.4 & 2.9 \\
\hline 2 & 1 & 2.8 & 2.5 \\
\hline 3 & 1 & 4.6 & 10.7 \\
\hline 4 & 1 & 2.5 & 5 \\
\hline 5 & 1 & 1.2 & - \\
\hline
\end{tabular}

Table 4: Gating ratios employed in each mold design. Note there is no value for ingate ratio in Mold 5 because this was the top-feeding system.

\subsection{X-Ray Radiography Setup}

The setup for the casting experimentation included the mold, a pouring mechanism and the X-ray unit. On top of the mold, an insulating pouring basin was filled with approximately $2.1 \mathrm{~kg}$ of aluminum with a 356 composition. Then, via remote activation, a graphite stopper rod was removed from the bottom of the basin to allow metal to flow into the mold. Simultaneously, a Charged Couple Device (CCD) camera operating at 30 ferrules per second (fps) began recording X-ray images as the metal filled the mold, as shown in Figures 21 and 22. 


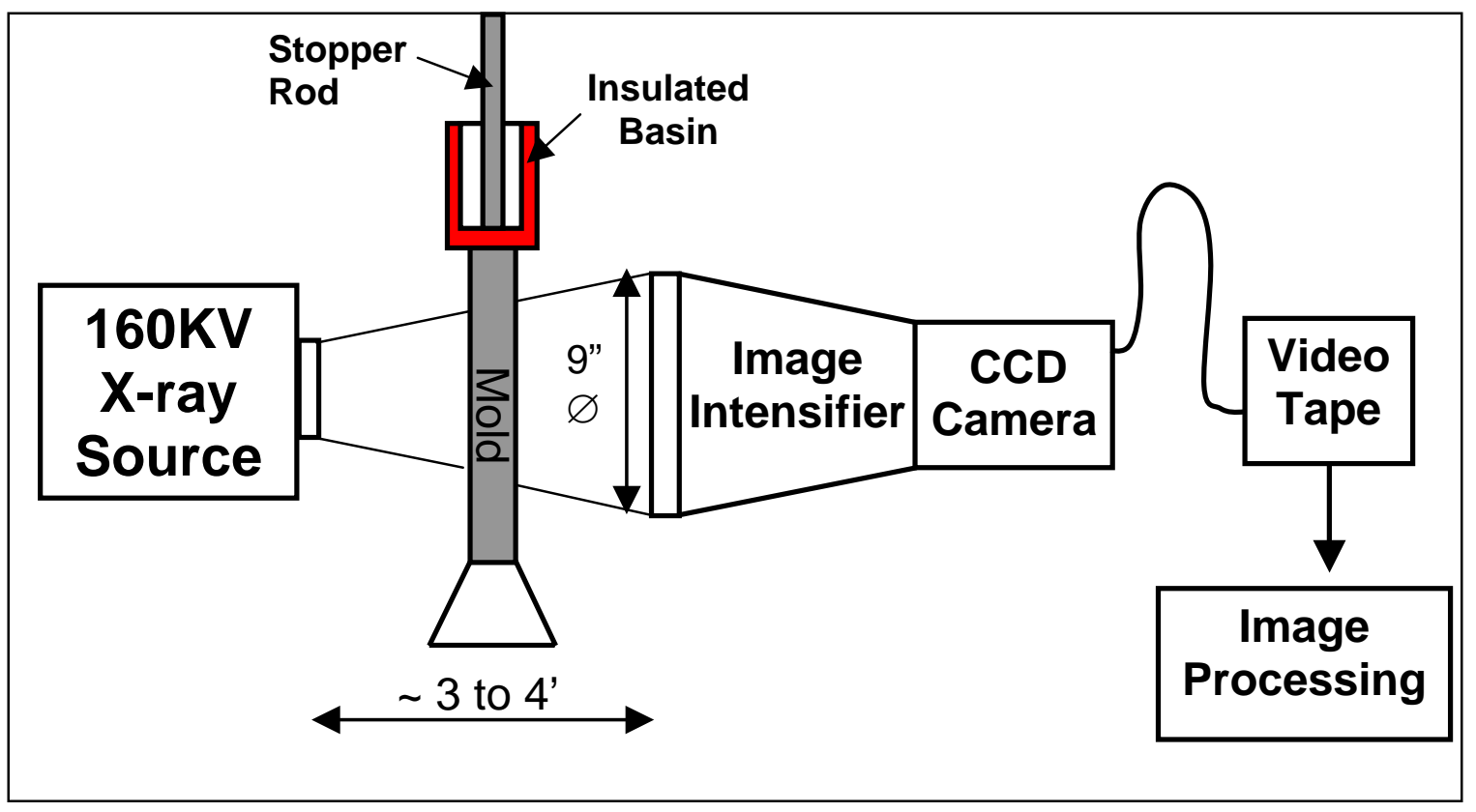

Figure 21: Schematic of X-ray setup. 


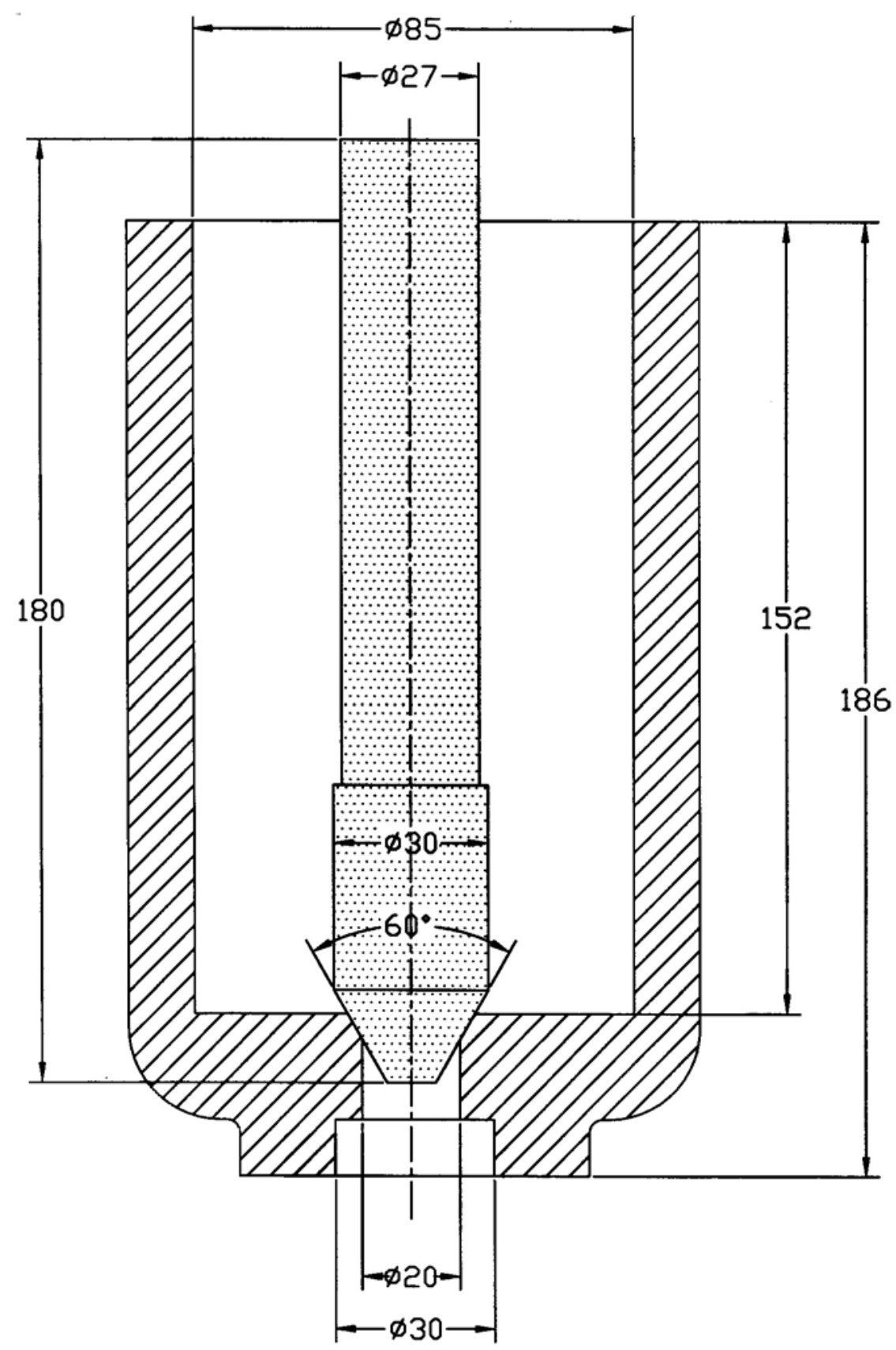

Figure 22: Schematic of insulated pouring basin. 


\subsection{Process Parameters}

To make the casting processes as consistent as possible, several parameters were defined prior to experimentation. First, the molds were preheated to $315^{\circ} \mathrm{C}\left(600^{\circ} \mathrm{F}\right)$ and the molten aluminum was brought to $815^{\circ} \mathrm{C}\left(1500^{\circ} \mathrm{F}\right)$. When the appropriate temperatures were reached in the metal and the mold, the molten aluminum was transferred to the insulated pouring basin. The initial height of the metal in the basin was $14 \mathrm{~cm}$ ( $5.5 \mathrm{in}$ ) with the stopper rod in place. This amounts to $775 \mathrm{~cm}^{3}$, or $2.1 \mathrm{~kg}(4.6$ lb)of molten aluminum. The known height establishes the head pressure for filling the mold (Equation 1). Upon exiting the X-ray room, the real-time $\mathrm{X}$-ray radiography was started while the stopper rod was simultaneously removed. The fill time for this operation varied between molds, but ranged from $1.2-1.7$ seconds.

\subsection{MAGMAsoft Simulation}

Once the real-time videos were produced, simulations using MAGMAsoft computer simulation software were used to obtain fill patterns. By setting various options in MAGMAsoft, like mold and metal temperature, head pressures at various points in time, thermal properties and interactions between the mold and metal, etc., filling models were produced to examine overall fill patterns. This was done with the following procedure.

A meshed model was produced from the bulk geometry using MAGMAsoft's automatic 
mesh generating module. A screenshot of the configuration page is shown in Figure 23. Time considerations dictated a 2000000 node limit.

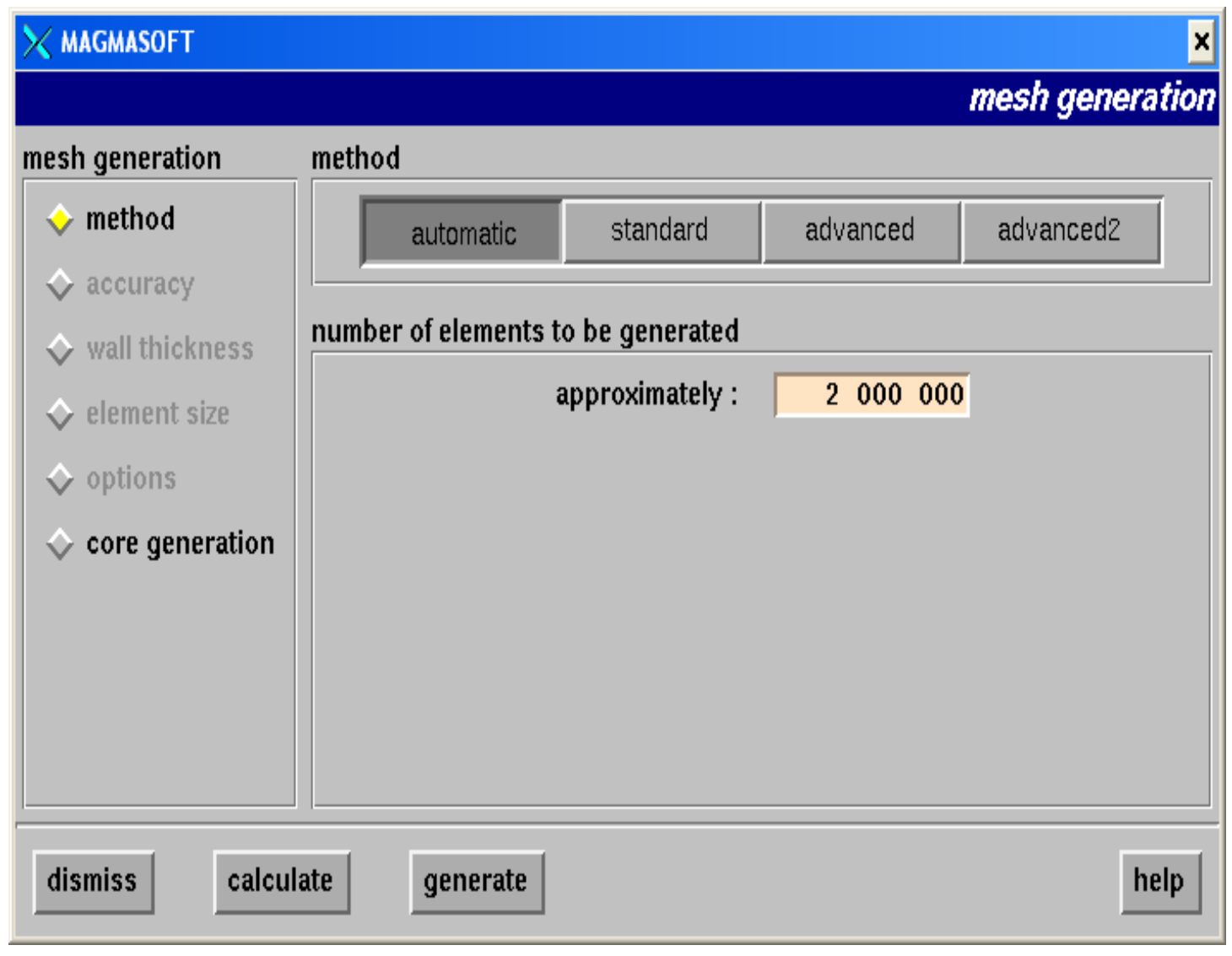

Figure 23: Screenshot of MAGMAsoft automatic enmeshment module. 
The mold and casting materials were identified, from which MAGMA extracted data from its built-in database for various material properties. These included viscosity versus temperature, melting temperature, heat capacity, etc. Examples of this portion of the software can be seen in Figure 24.

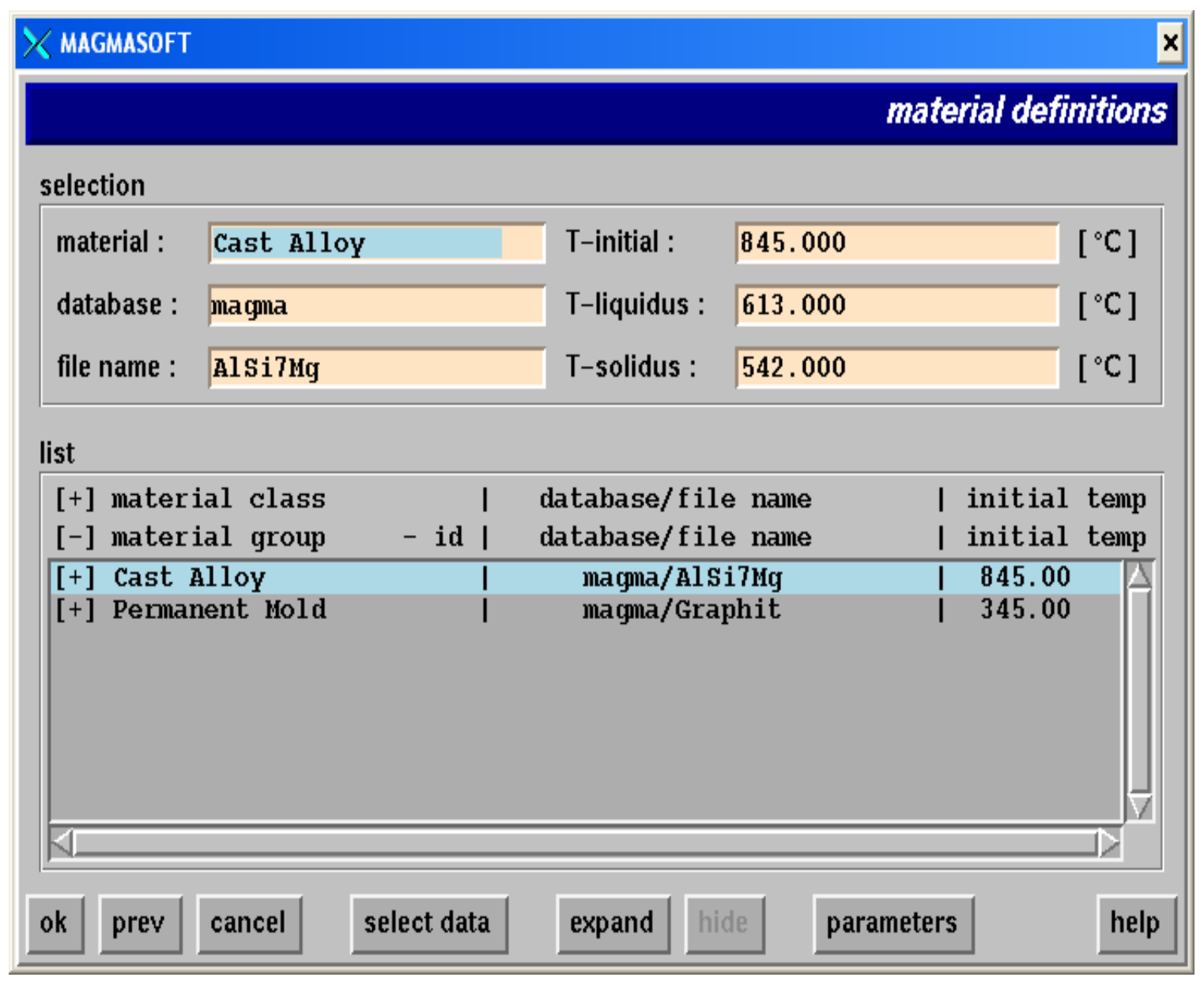

Figure 24: Screenshot of material identification entry. 
The temperature of the aluminum and mold at the time of casting were entered as $815^{\circ} \mathrm{C}$ and $315^{\circ} \mathrm{C}$, respectively. Figure 25 shows a screenshot of this stage.

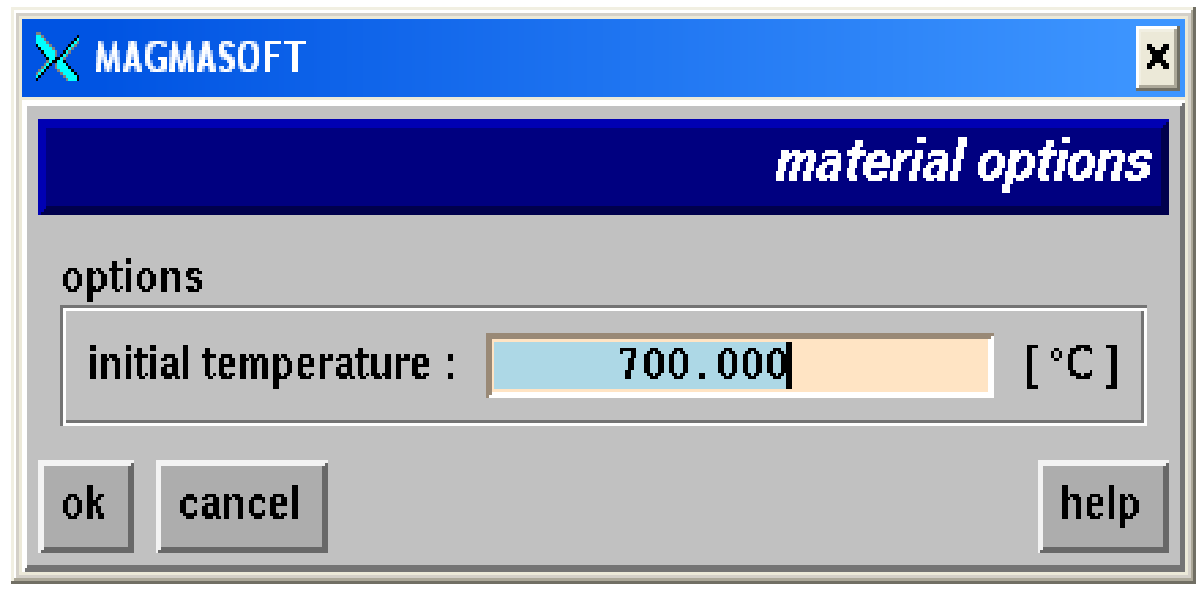

Figure 25: Screenshot of initial temperature customization. 
A "heat transfer" coefficient, calculated by the MAGMAsoft software, was determined for the system. For this system, a heat transfer definition was calculated for “AlSi7Mg Mold”. Figure 26 shows a screenshot of the heat transfer definition options.

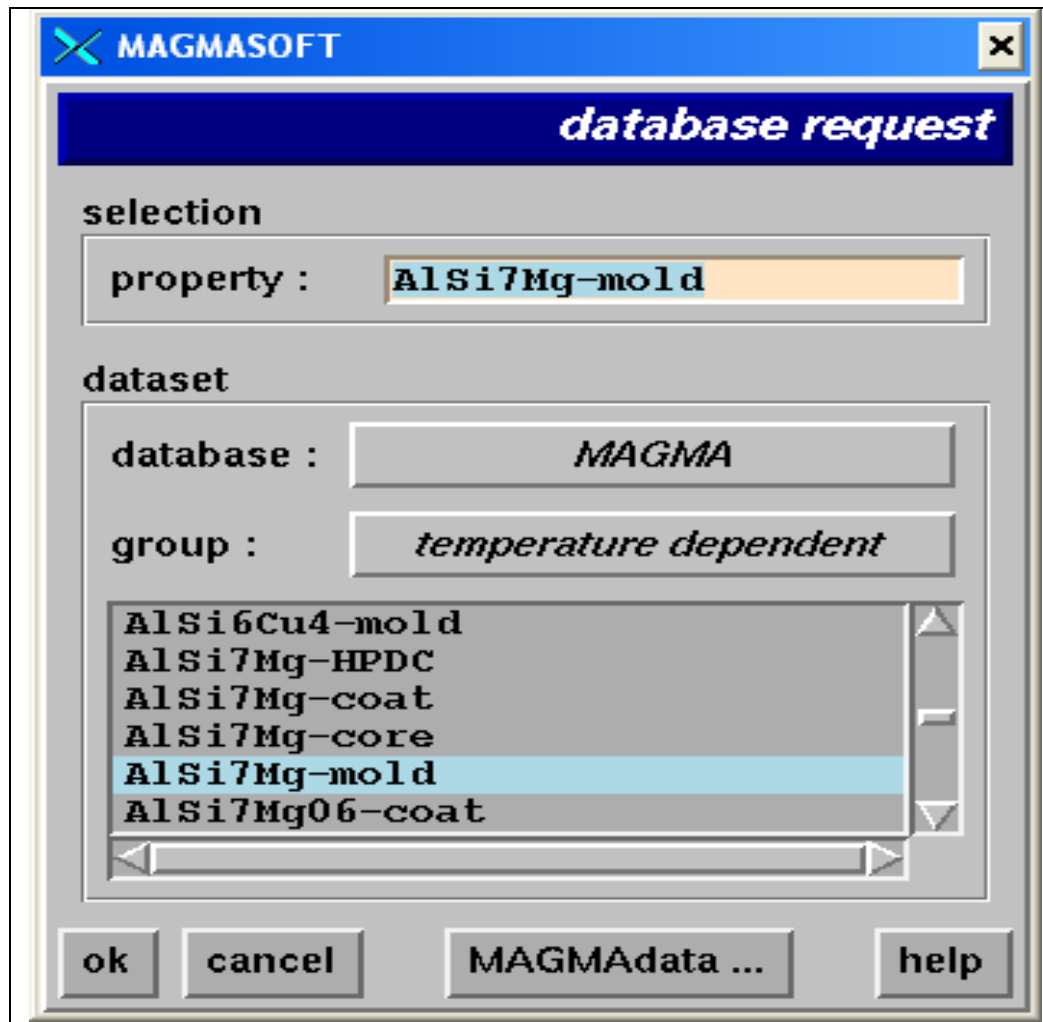

Figure 26: Screenshot of heat transfer definition options. 
If a filter was used, the filter type was identified as Ceramic Foam, 10 ppi. Also, MAGMAsoft allows the filter to be "activated" or "deactivated". This way, tests can be performed on fluid flow through the empty filter print to observe any differences with or without the filter. A screenshot of this setting can be found in Figure 27.

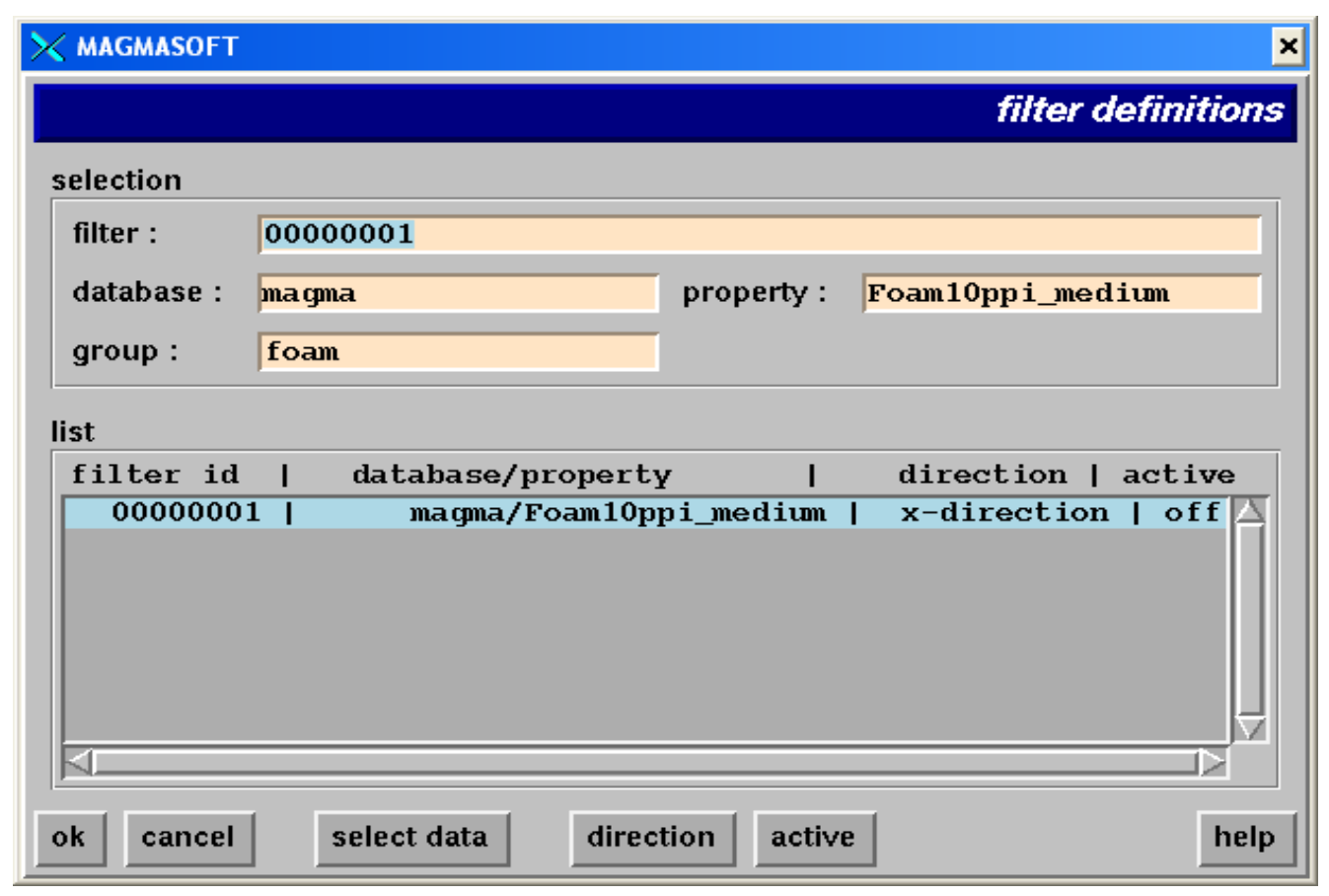

Figure 27: Screenshot of filter setup.

The calculations can be driven by fill time, fill rate or pressure. For this experiment, head pressure at the top of the sprue was used. An approximated pressure versus time plot was generated with the assumption that venting in the molds is sufficient to prevent backpressure. Table 5 is the data used for the pressure vs. time plot. The calculations were made assuming a constant rate of pressure decline. 


\begin{tabular}{|r|r|}
\hline \multicolumn{1}{|c|}{ Time } & \multicolumn{1}{c|}{$\begin{array}{c}\text { Pressure } \\
\text { (s) }\end{array}$} \\
\hline 0.0 & 36.69 \\
\hline 0.1 & 33.87 \\
\hline 0.2 & 31.05 \\
\hline 0.3 & 28.23 \\
\hline 0.4 & 25.41 \\
\hline 0.5 & 22.59 \\
\hline 0.6 & 19.77 \\
\hline 0.7 & 16.95 \\
\hline 0.8 & 14.12 \\
\hline 0.9 & 11.30 \\
\hline 1.0 & 8.48 \\
\hline 1.1 & 5.66 \\
\hline 1.2 & 2.84 \\
\hline 1.3 & 0.02 \\
\hline
\end{tabular}

Table 5: Data used for pressure versus time definitions

With this information, the simulation was started. Several iterations were necessary in some situations, where settings needed to be modified to find a good match between the video and computer simulation. This process was performed for all mold configurations. After a close match between videos and simulations was achieved, zero-mass tracer particles were electronically placed in the sprue of the casting models. When the simulations were run once again, the tracer particles showed flow patterns and trends inside the metal, rather than just the outer surface. It was assumed that the tracer particle simulations were accurate since the outer surface representations were similar to the videos. 


\subsection{RESULTS AND DISCUSSION}

\subsection{Gating Section Behavior}

\subsubsection{Sprue}

As expected, the metal accelerates as it drops down the sprue. The taper of the sprue does lessen the affect of gravity in accelerating the metal. Figure 28 shows images of the recorded X-ray radiography for Mold 1. Figures 29 shows tracer simulation images at different times as the sprue fills for Mold 1.

At the base of the sprue, when no sprue-well is present, a vortex forms near the closed corner of the sprue, or side opposite the gating. As the metal stream falls and strikes the base of the sprue, the stream separates to the right and left. The left side forms a circular path and begins to redirect the falling metal stream into the gating area. This does not appear to affect the velocity of the metal, but does reduce turbulence with less splashing than associated with the metal abruptly hitting the bottom of the sprue. 


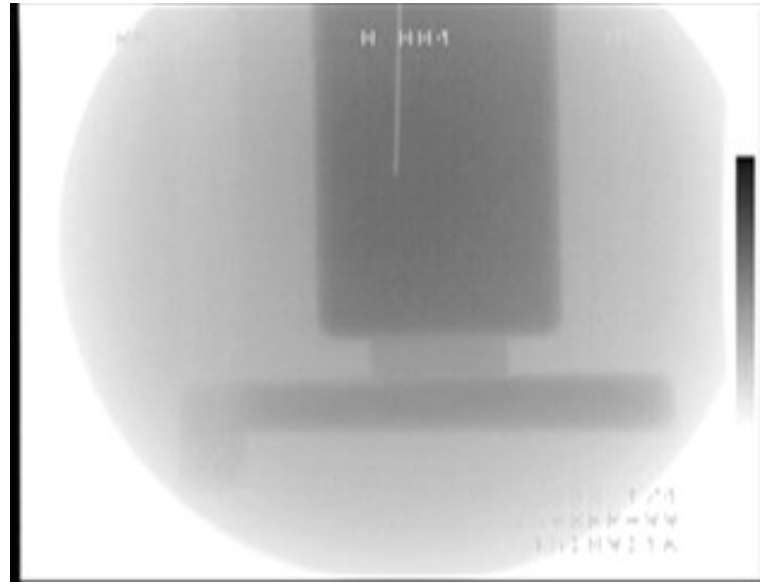

(a)

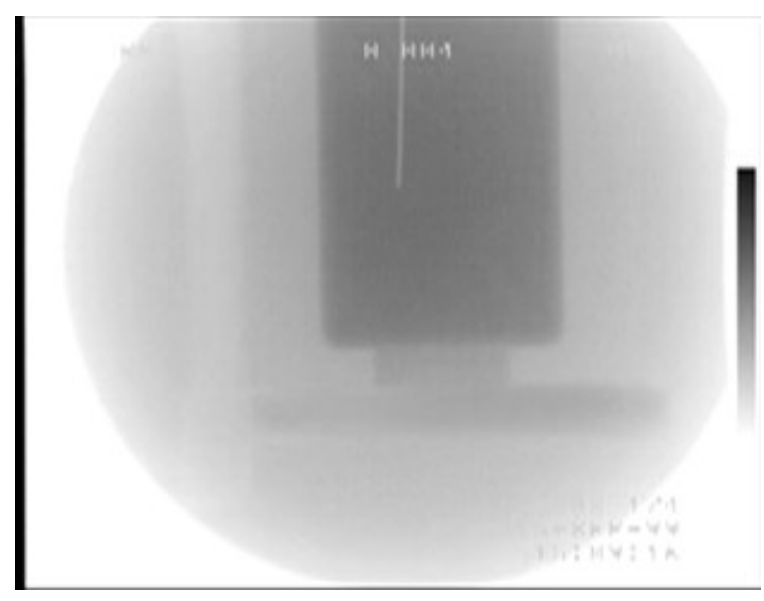

(b)

Figure 28: Video image sequence from Mold 1 depicting sprue behavior.

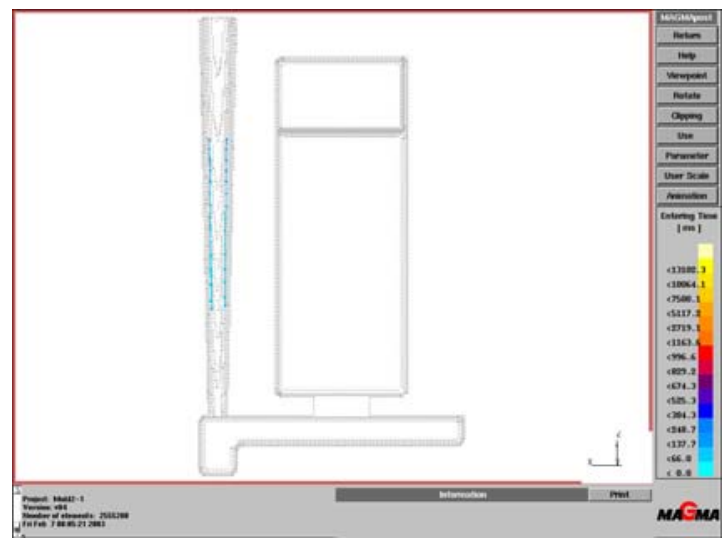

(a)

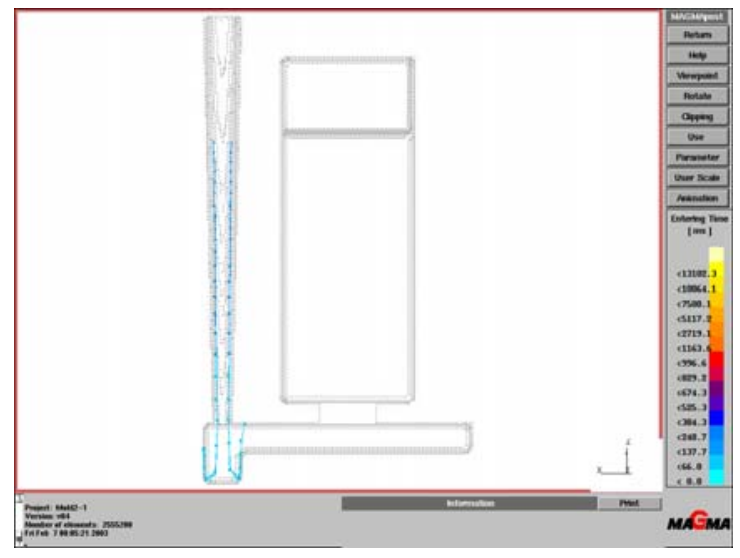

(b)

Figure 29: Tracer simulation sequence for Mold 1 depicting sprue behavior. 


\subsubsection{Sprue-Well}

Many of the simulations show that the sprue-well fills rapidly. As the basin begins to fill, two paths are followed by the metal. The portion closer to the gating proceeds into the gating of the mold. The other half begins to circulate in a "whirlpool" fashion as demonstrated in Figure 30. This behavior is very similar to the situation presented in a sprue without a basin. After the basin fills, the flow path of the metal shifts noticeably. With the basin filled, metal starts to forgo the basin and the flow acts as if no sprue-well is present at all. The swirling affect can lead to a loss of kinetic energy. The remaining energy is insufficient to overcome the forces caused by the stream of metal flowing down the sprue. Enough energy remains to push the stream toward the open gating system. This modifies the dynamics of the mold filling because the sprue-well acts as though it longer exists. The manner in which the metal stream is affected causes a vena contracta to form unless a filter is present. The case of systems without a filter will be discussed later. Examples of vena contracta formation during filling can be seen in both the video samples and simulation models as shown in Figures 31 and 32. Previously presented schematics show that the sprue-well was designed with the generally accepted dimensions. Therefore, this phenomenon may be common in many gating systems designed with these principles in mind. 


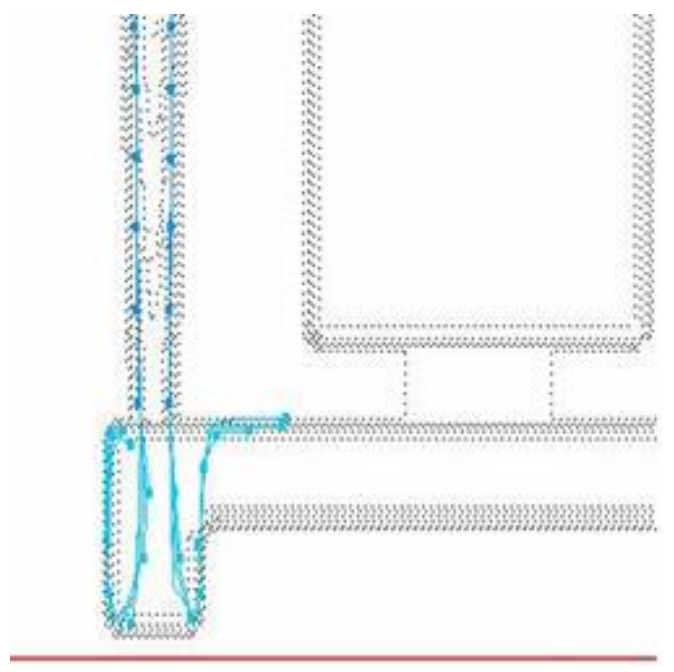

(a)

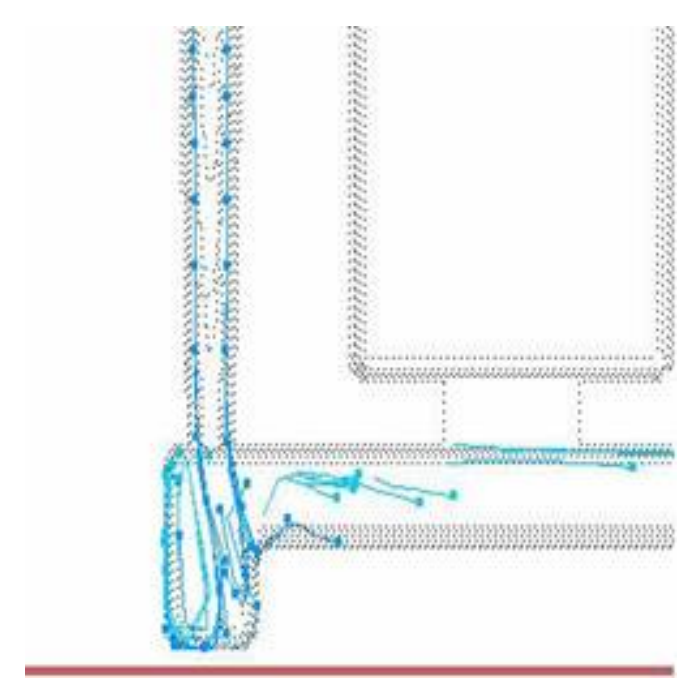

(c)

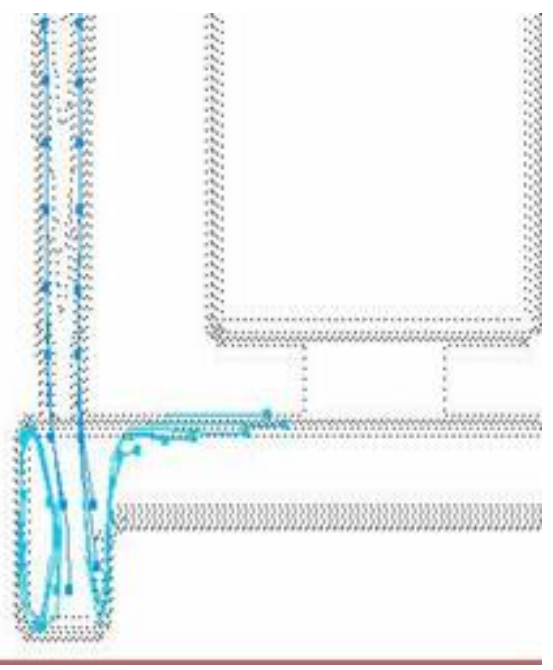

(b)

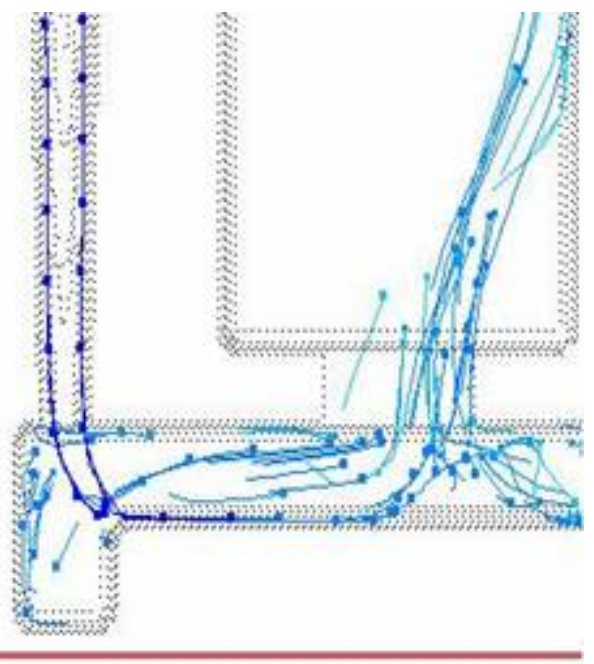

(d)

Figure 30: Image sequence of tracer simulation depicting sprue well circulation behavior. 


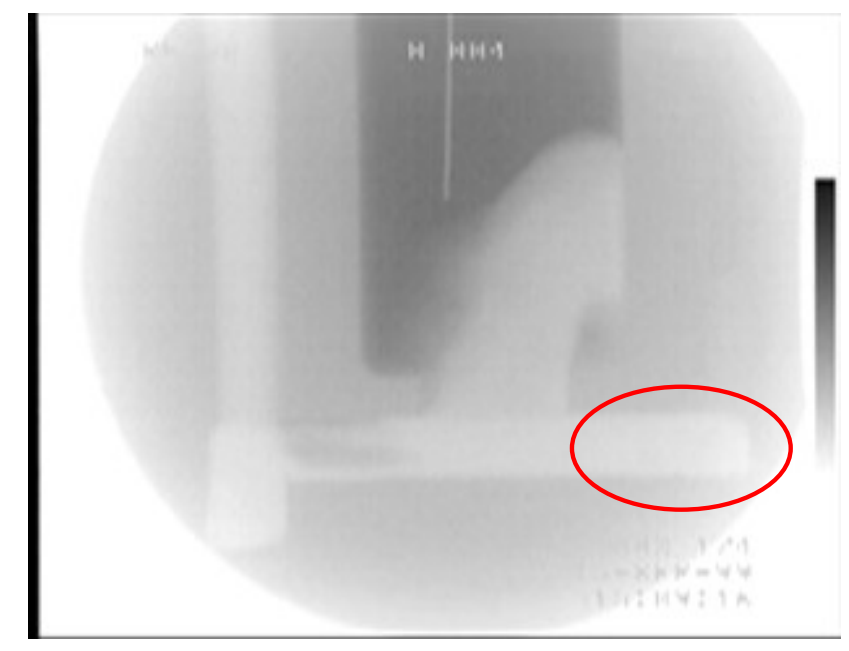

(a)

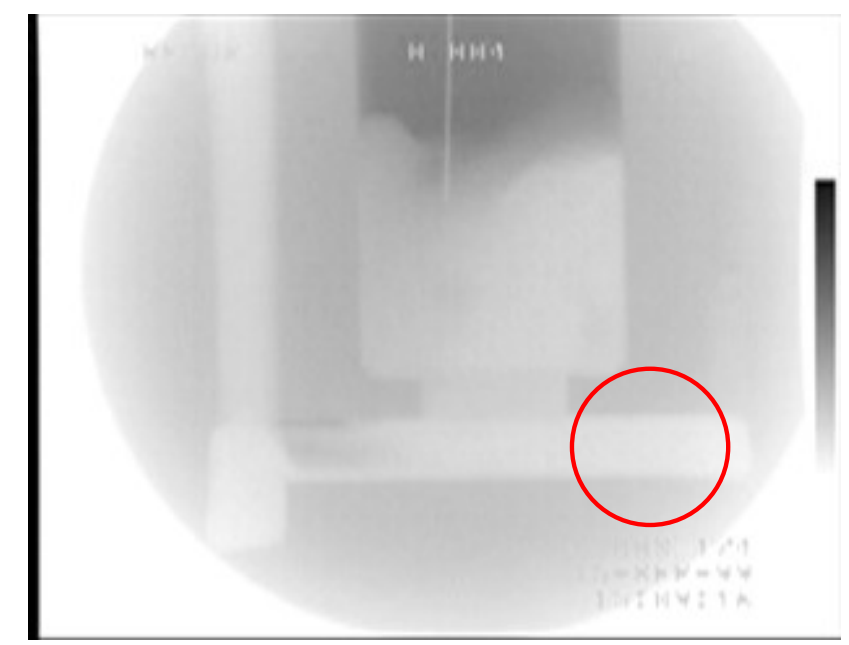

(b)

Figure 31: X-ray image sequence depicting formation of vena contracta in Mold 1. 


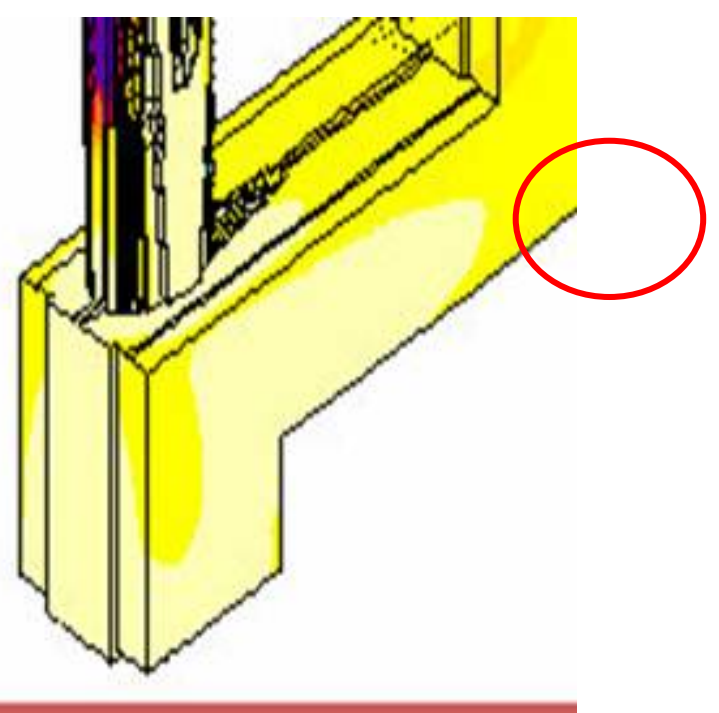

(a)

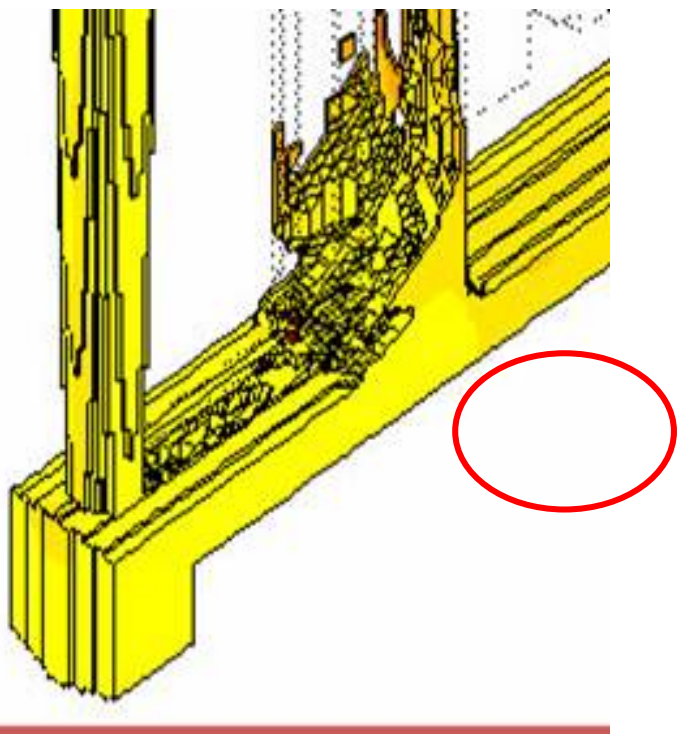

(b)

Figure 32: Simulation images showing the formation/presence of vena contracta in (a) Mold 1 and (b) Mold 4. 


\subsubsection{Feeder}

Mold 4 has a feeder incorporated in a side-feeding gating system. As noted from both the video and simulation images in Figures 33 and 34, much of the feeder filled prior to metal entering the mold cavity through the thin ingate. This indicates that a threshold head pressure is necessary to overcome the frictional forces generated by the very thin ingate. Filling of the feeder reduced the turbulence but as the metal enters into the mold cavity through the ingate, a rather significant metal velocity was observed. This happens at the base of the mold cavity and the filling of the mold occurs gradually and uniformly.

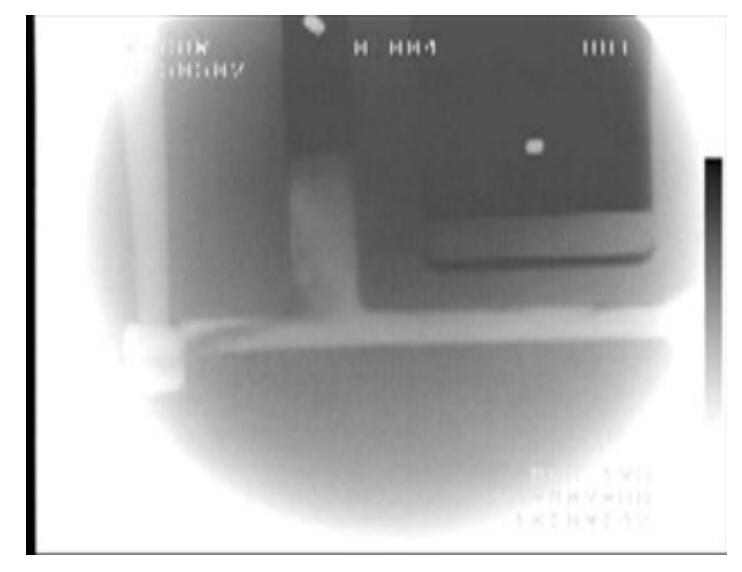

(a)

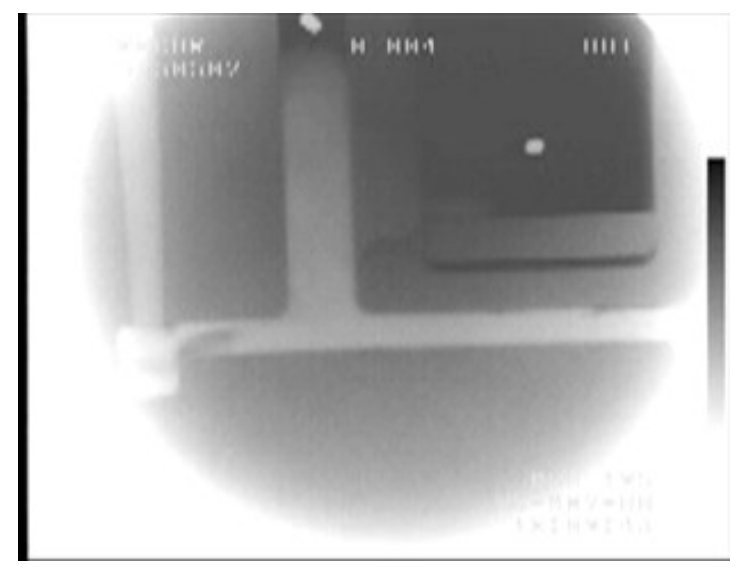

(b)

Figure 33: X-ray image sequence showing filling of filler in Mold 4. 


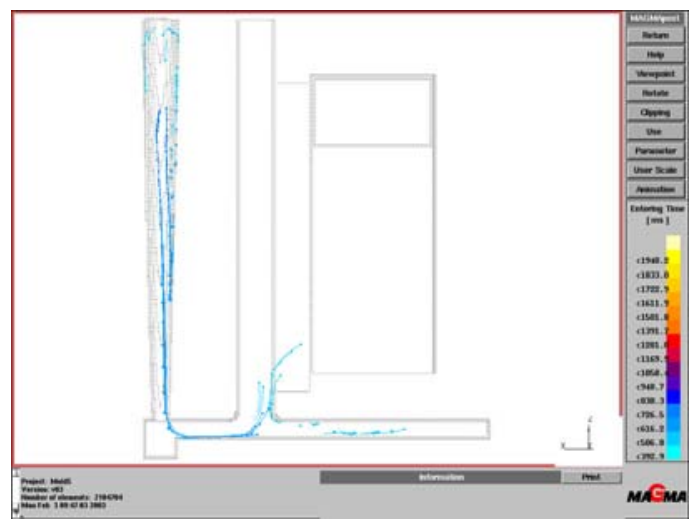

(a)

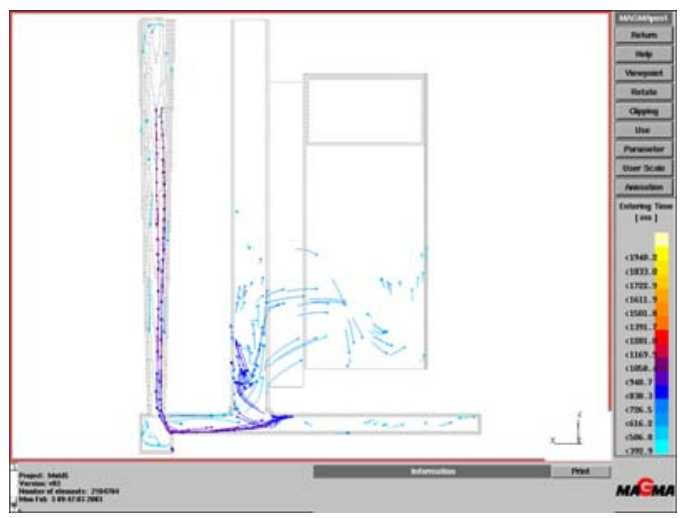

(c)

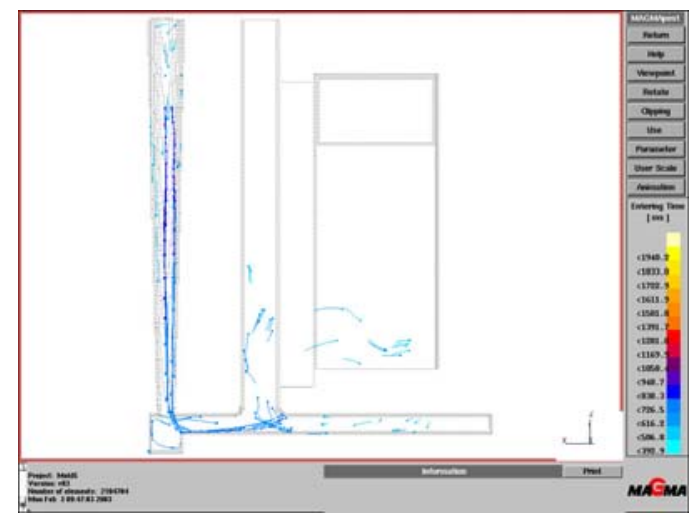

(b)

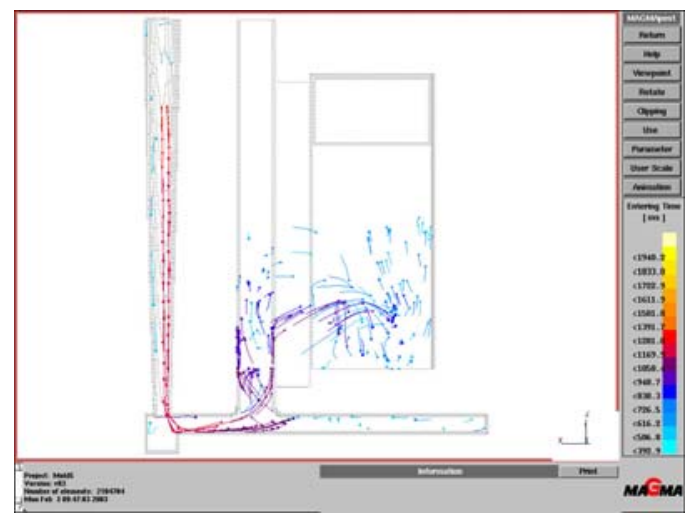

(d)

Figure 34: Tracer simulation image sequence showing filling of feeder in Mold 4. 


\subsubsection{Filter}

Two molds, Mold 3 and Mold 5, utilize filters. For both of these molds, simulations were run with and without a filter in the print area. This was done to show exactly how the filters affect fluid flow in castings. In Mold 3, the filter was placed vertically immediately after the ingate in a bottom-feeding system. Because of the placement of the filter, it was assumed that a sprue-well was not needed since the filter should slow the fluid flow sufficiently to prevent the formation of vena contracta. Both the video and tracer simulation images in Figures 35 and 36 show a circulation action occurring between the base of the sprue and the filter. As metal enters the filter, the metal begins to move up, through the filter before it exits and then enters the remainder of the gating. This circulation appears to reduce the violence and may produce air bubbles and slag. However, this happens before the metal enters the filter and therefore any slag formed should be removed. 


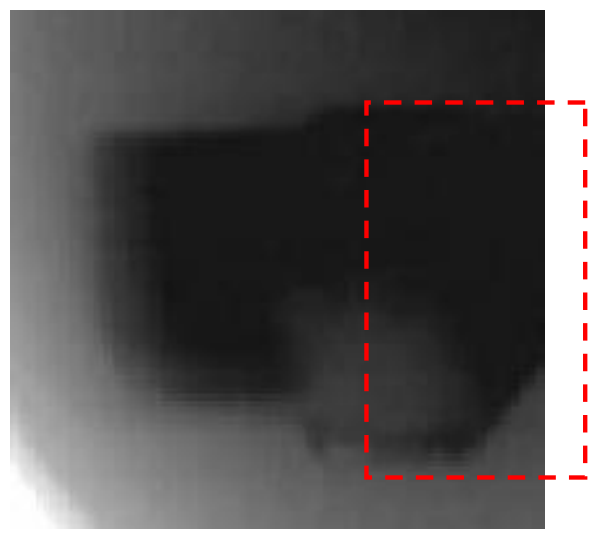

(a)

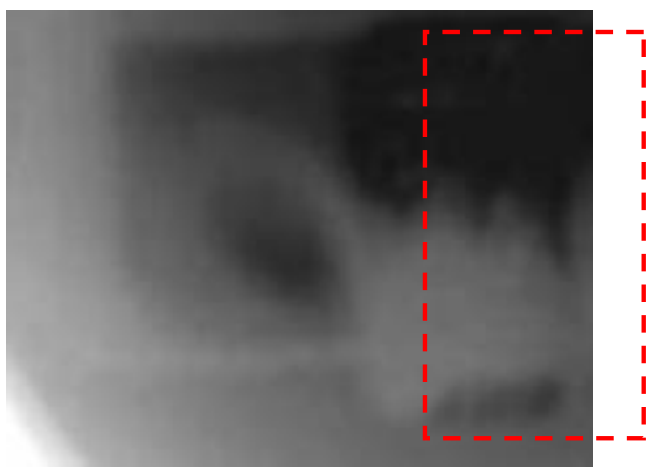

(c)

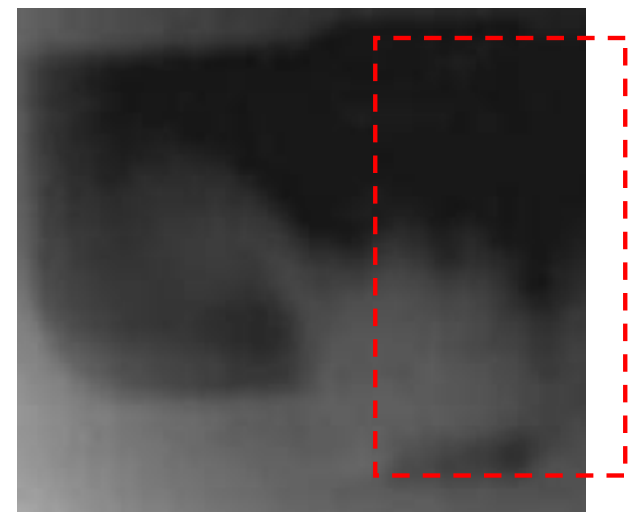

(b)

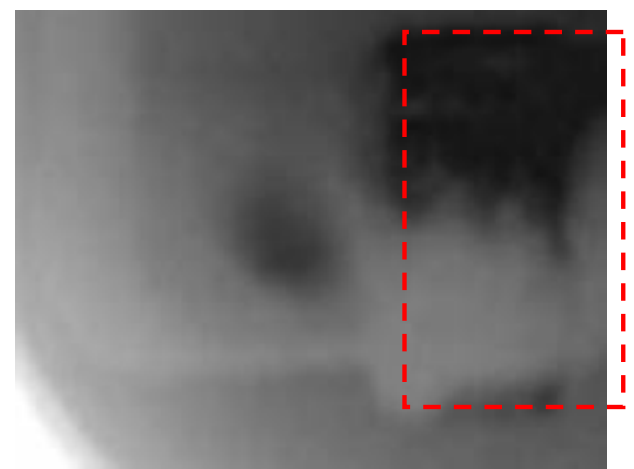

(d)

Figure 35: X-ray image sequence of circulation movement near filter in Mold 3. Note that the red box indicates the filter. 


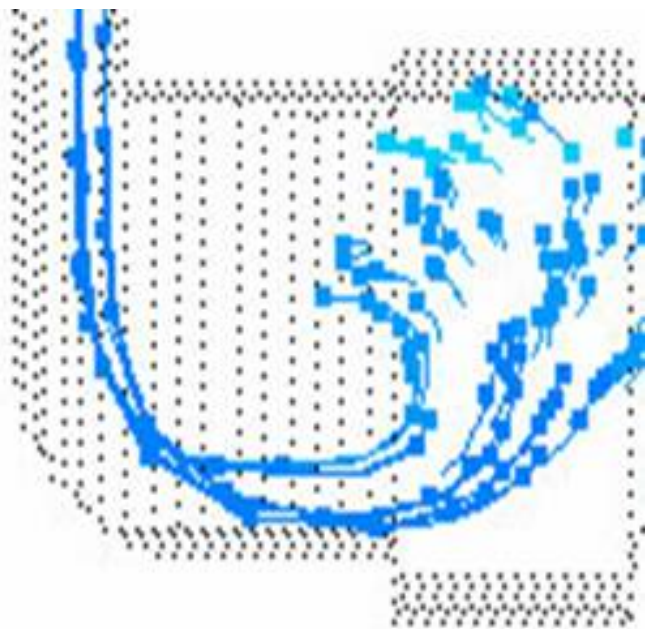

(a)

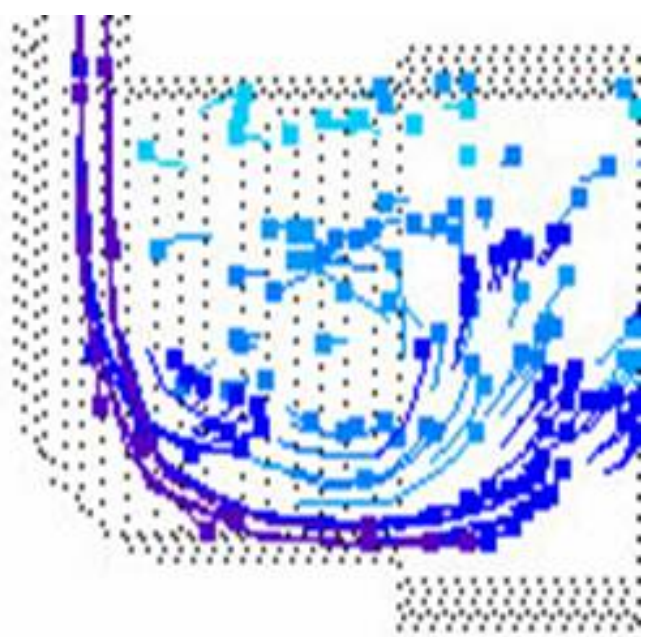

(c)

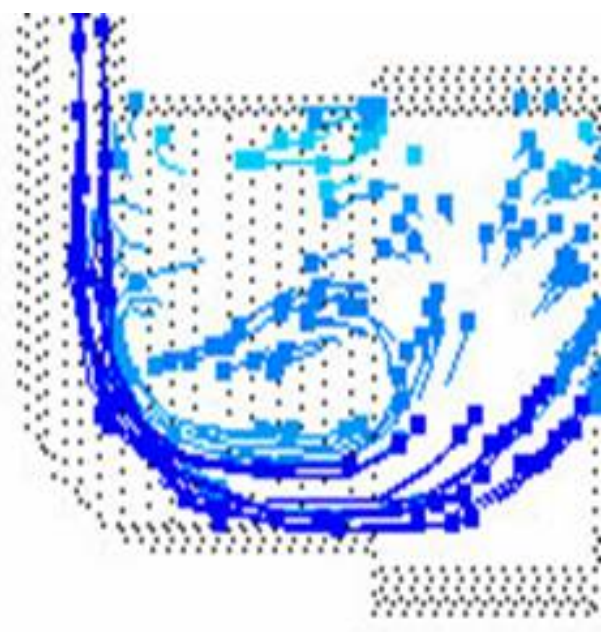

(b)

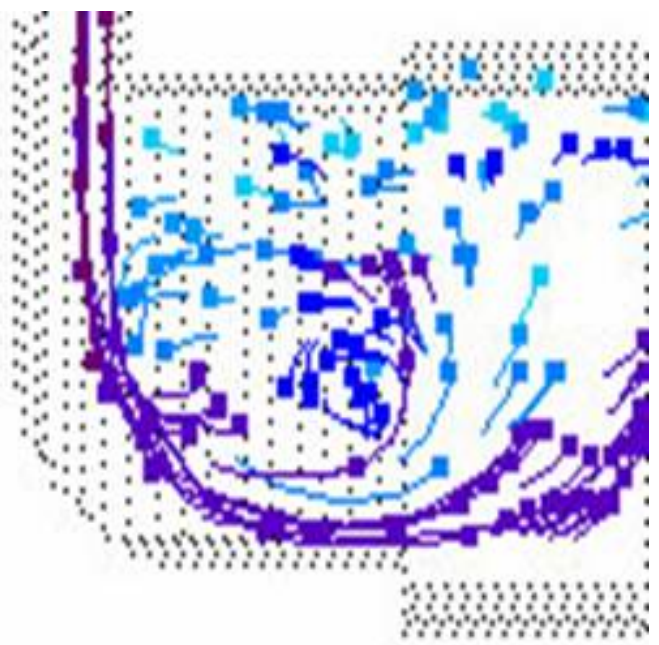

(d)

Figure 36: Tracer simulation sequence of circulation movement near filter in Mold 3. 
In Mold 5, the filter was placed vertically in a top-feeding system. When the filter is in place, much like in Mold 3, the filter begins to fill from the bottom before metal leaves the filter. Unlike the bottom-feeding system, no metal circulating prior to the filter was noticed in this system. After the metal exits the filter, the velocity is significantly reduced, as can be seen when comparing the metal stream in free-fall to the cavity base in Figure 37. Contrary to most systems, the decrease in velocity for a topfeeding mold may not be as beneficial to the casting. In the bottom-feeding mold, the lower velocity results in a much less turbulent mold filling when compared to the flow without a filter in the print. In the top-feeding mold, a drop occurs before the metal hits the side of the mold cavity. Therefore, the velocity of the metal is higher as it hits the bottom. This results in the metal moving back up the opposite side of the mold cavity, as seen in Figure 38, before filling the bottom of the mold. When no filter is present, the stream of metal hits the opposite side of the mold cavity very quickly and appears to cause the metal stream to spread instead of following the mold contour. In this case, the mold fills very uniformly across the bottom as shown in Figure 39. 


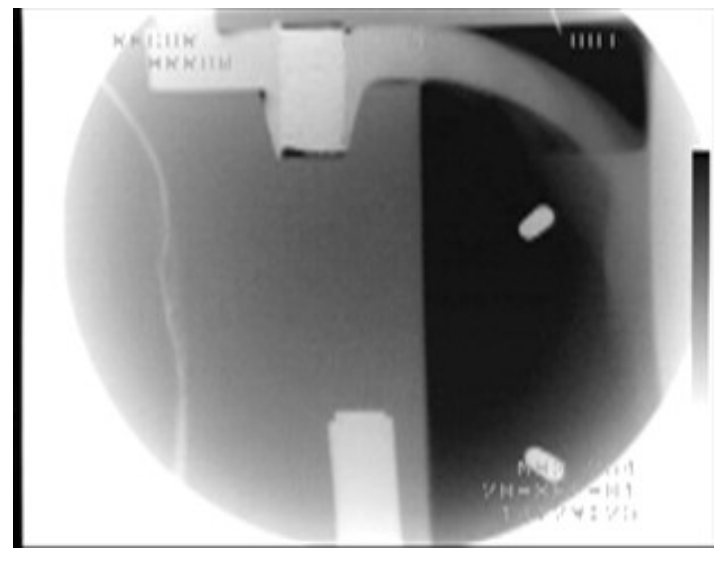

(a)

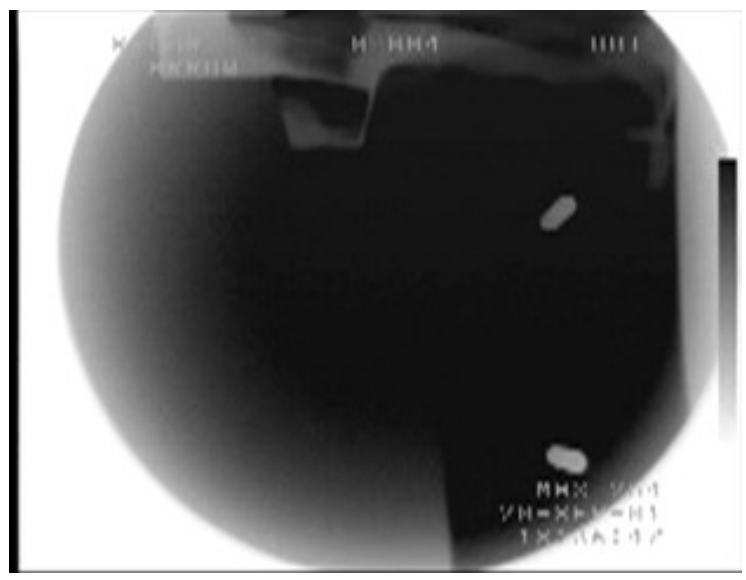

(b)

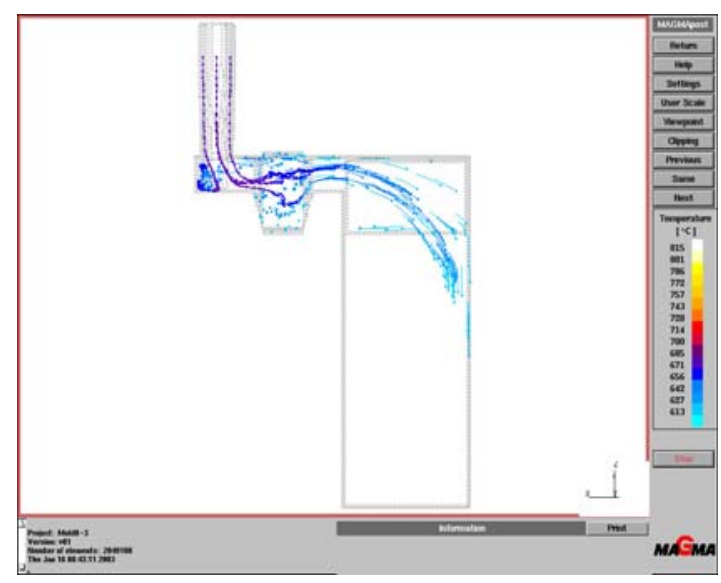

(c)

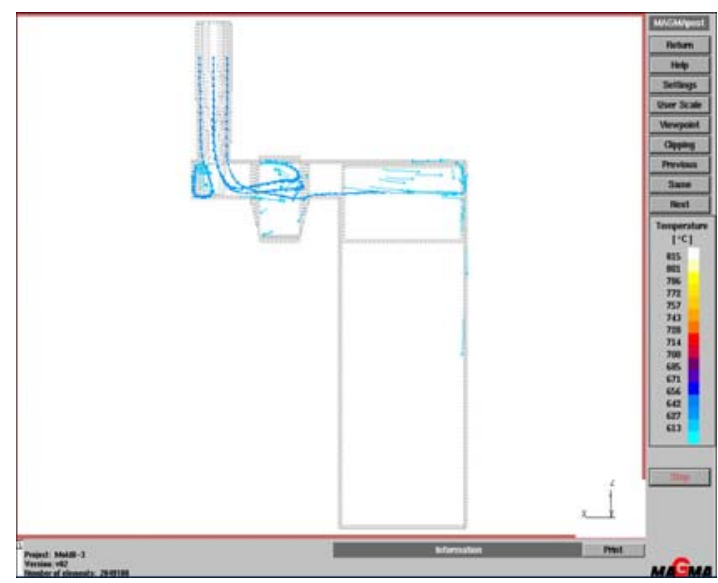

(d)

Figure 37: (a) X-ray image of metal stream in Mold 5 with filter.

(b) X-ray image of metal stream in Mold 5 without filter.

(c) Tracer simulation of metal stream in Mold 5 with filter.

(d) Tracer simulation of metal stream in Mold 5 without filter. 


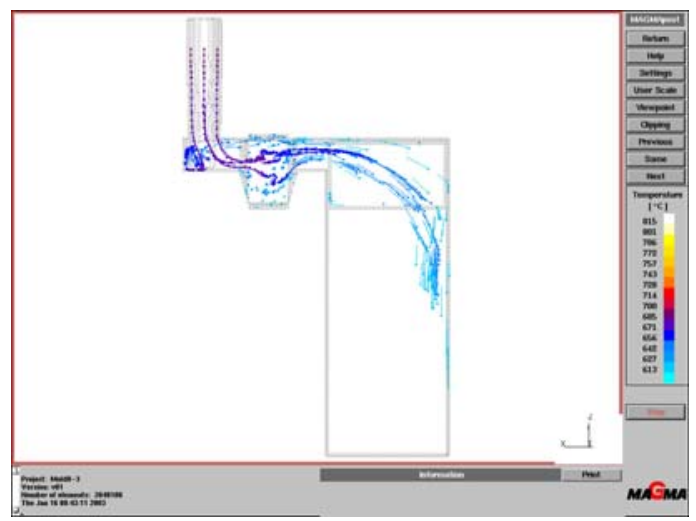

(a)

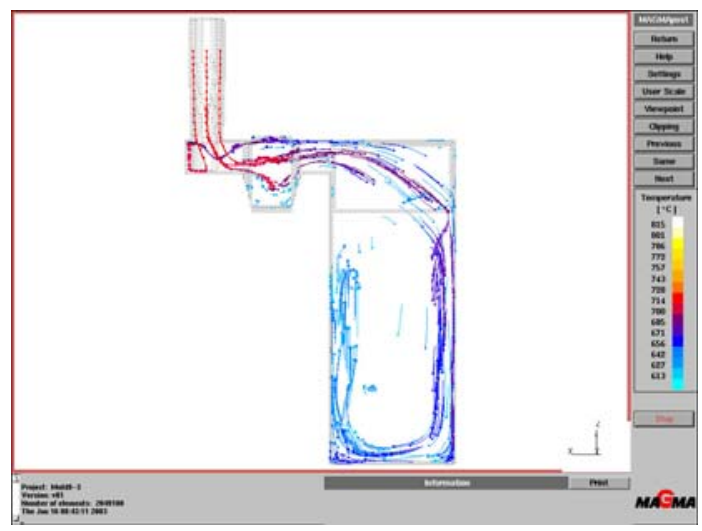

(c)

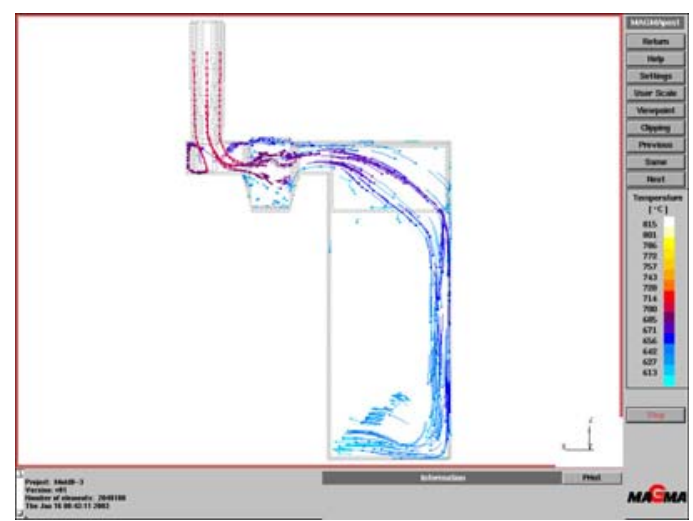

(b)

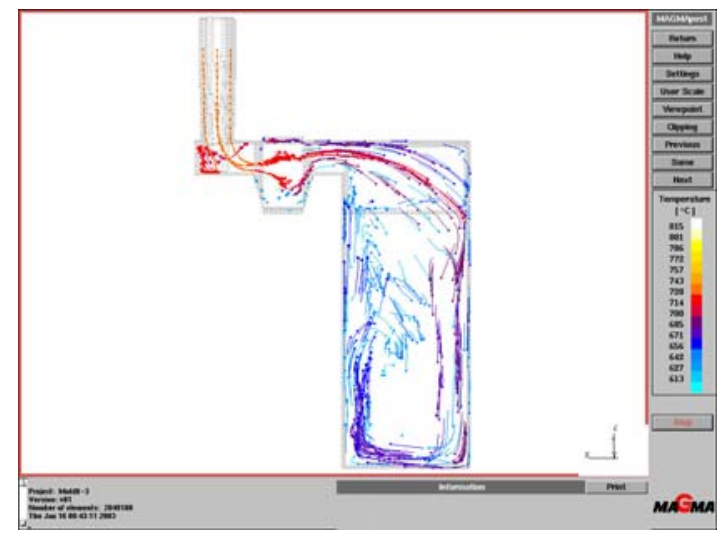

(d)

Figure 38: Tracer simulation sequence of metal climb in Mold 5 with a filter. 


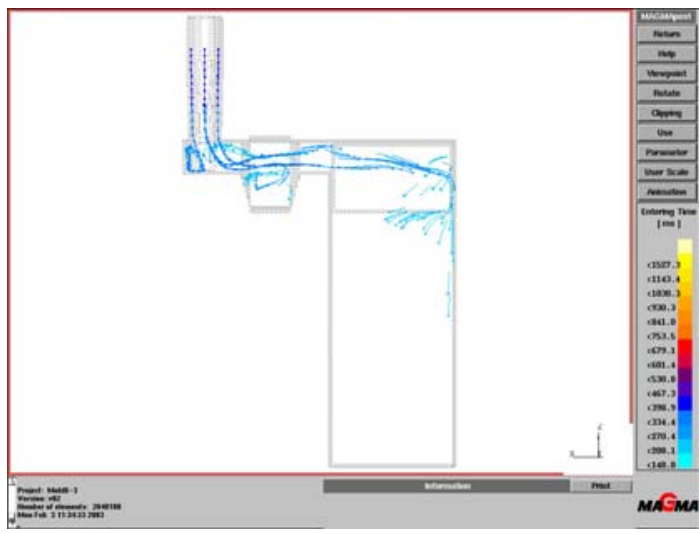

(a)

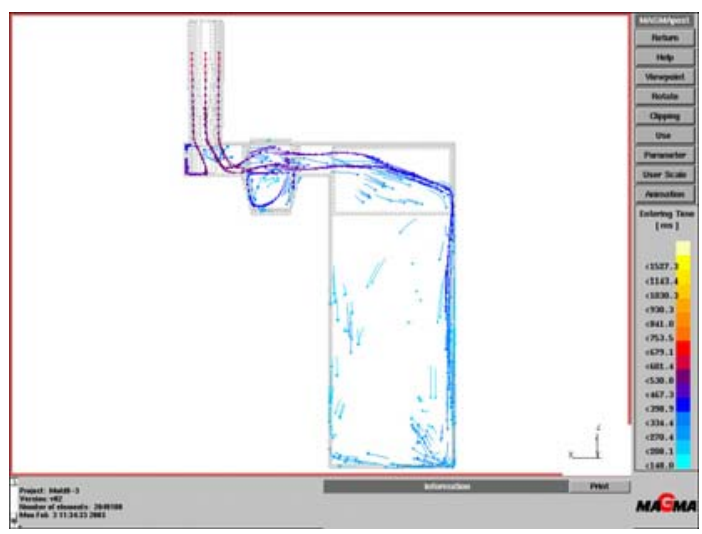

(c)

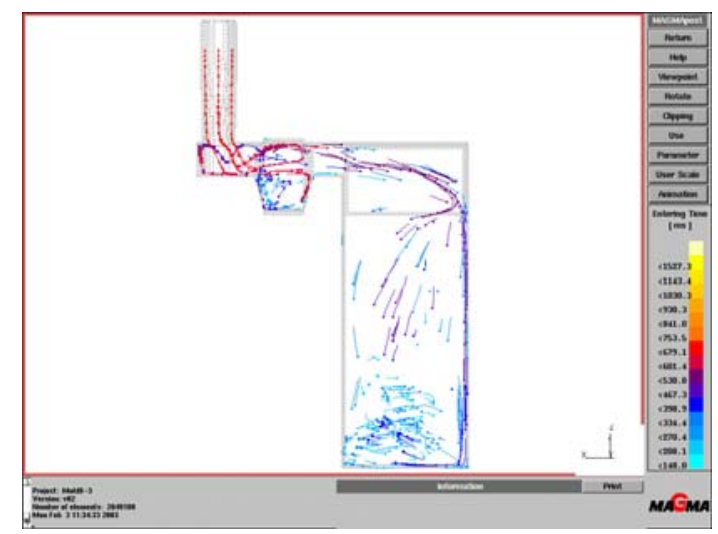

(d)

Figure 39: Tracer simulation sequence of metal climb in Mold 5 without a filter. 


\subsubsection{Runner}

In these experiments, all molds had runner extensions except the top-feeding Mold 5. Molds 2 and 3 had tapered extensions with large cross-sectional areas but Mold 3 incorporated a filter. Mold 1 had a squared extension with a smaller cross-sectional area in a bottom-feeding system, and Mold 4 had a similar runner extension used in a side-feeding system.

Generally, all extension designs trapped the first metal to enter the mold initially. However, in the case of the larger cross-sectioned extensions in Molds 2 and 3, the runners were too large to prevent the first metal from reentering the system and finding its way into the mold cavity. This can be seen by the number of light blue tracer particles that have entered the mold cavity in Figures 40 and 41. In the molds with squared runner extensions, the metal entered the mold cavity in a relatively violent fashion. This is confirmed by looking at the initial images of the X-ray videos shown in Figures 42 and 43. In the case of the tapered runner, the metal entering the system is less violent than the squared runner extension. Simulations also showed that more metal appeared trapped in the squared extension with a smaller cross-sectional area as shown by the larger amount of blue tracer particles in the runner extension. 


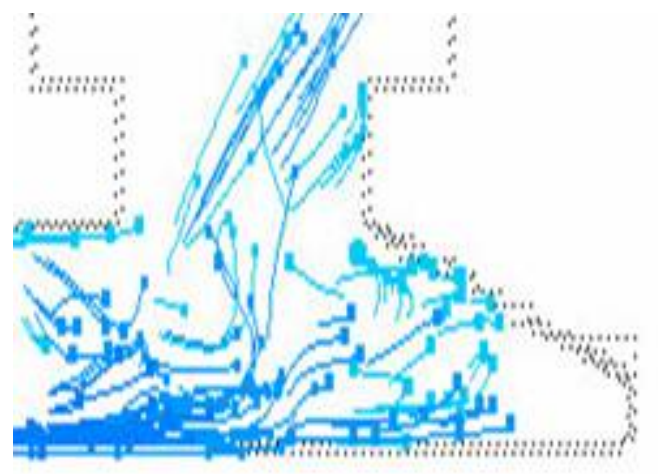

(a)

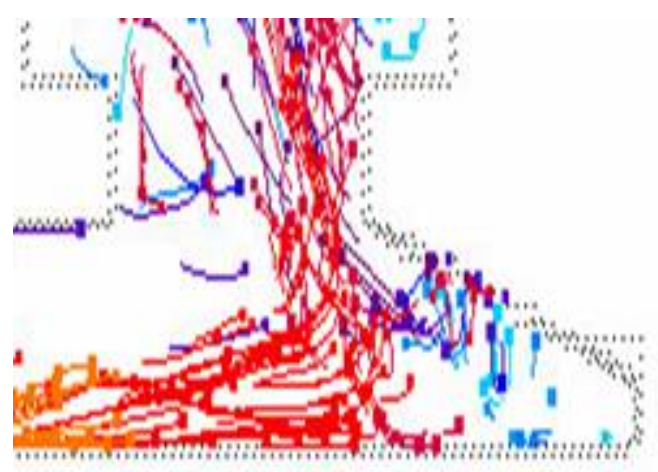

(c)

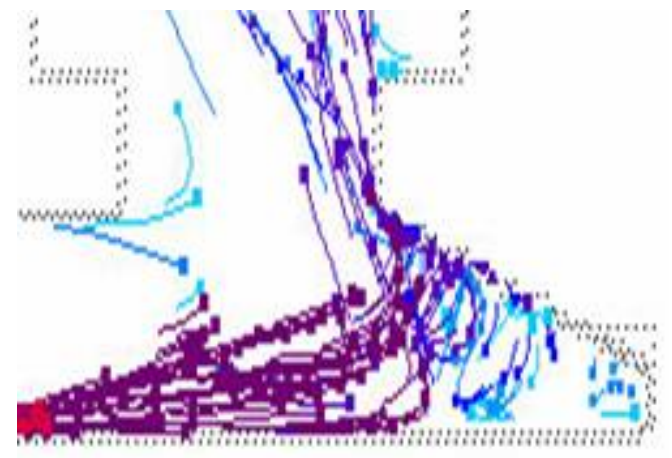

(b)

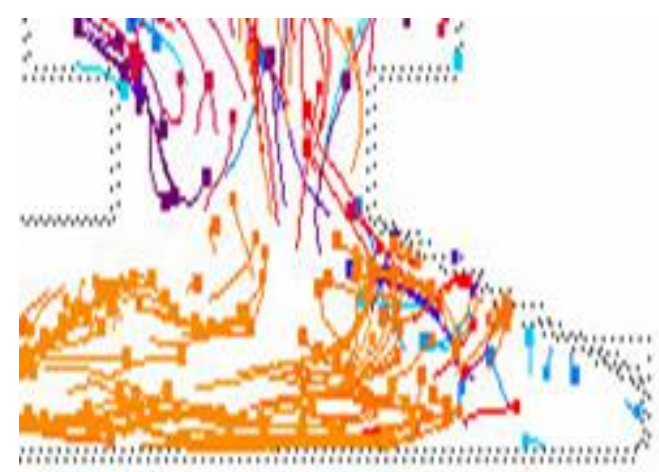

(d)

Figure 40: Tracer simulation sequence of first metal moving from the runner extension to the mold cavity in Mold 2. 


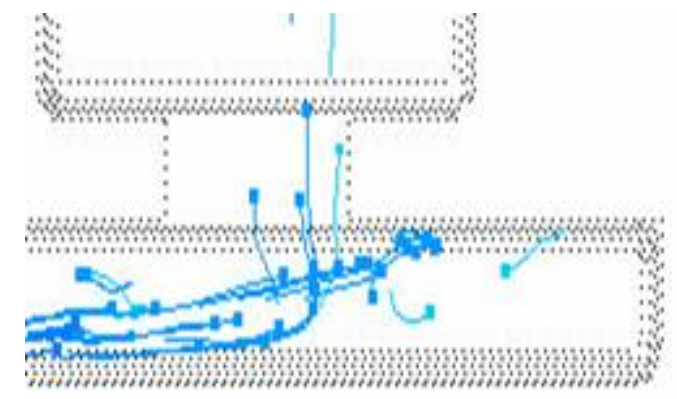

(a)

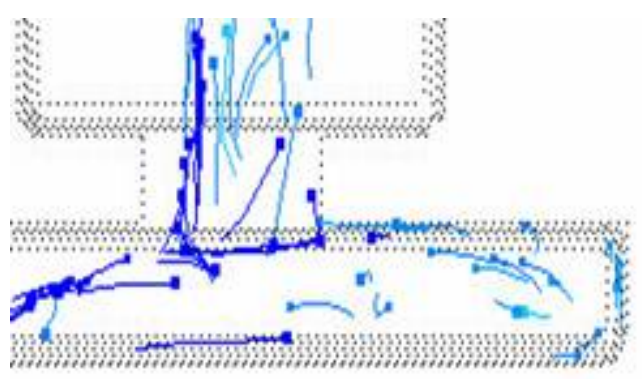

(c)

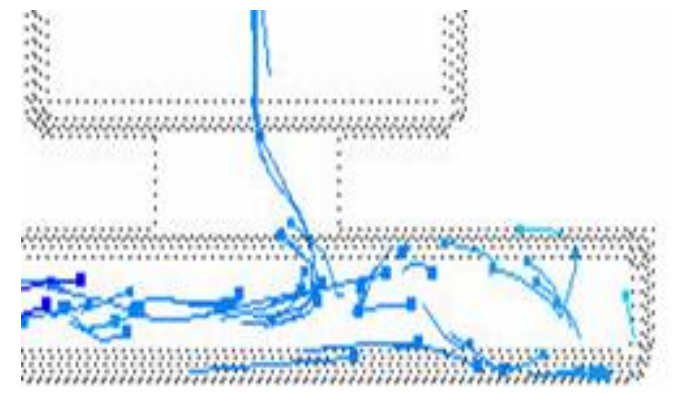

(b)

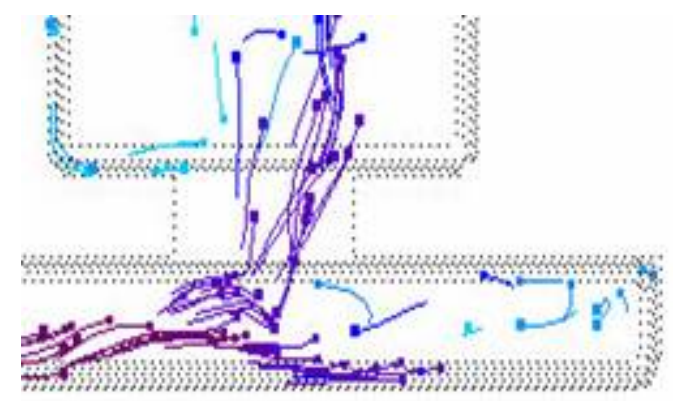

(d)

Figure 41: Tracer simulation sequence of first metal moving from the runner extension to the mold cavity in Mold 1. 


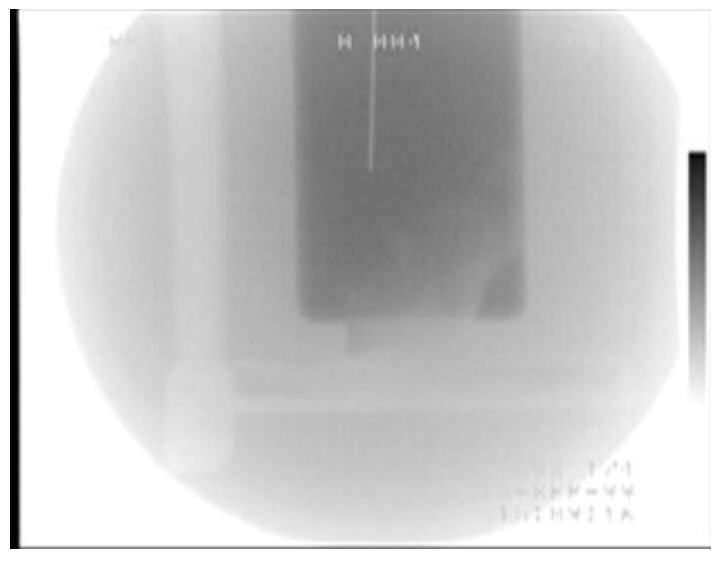

(a)

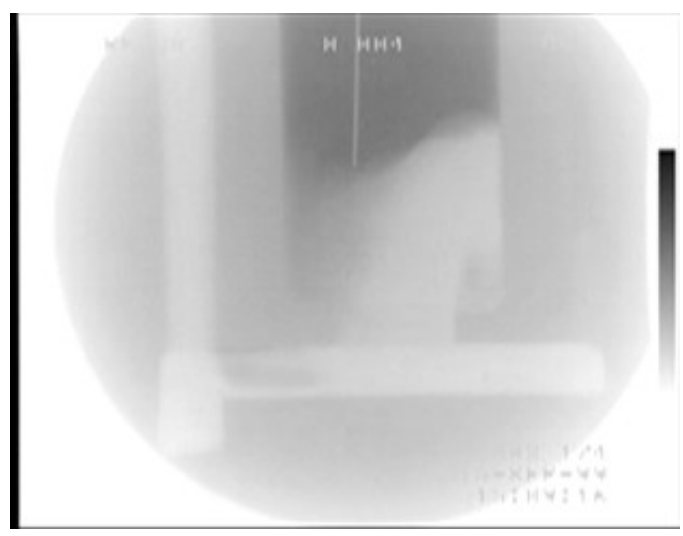

(c)

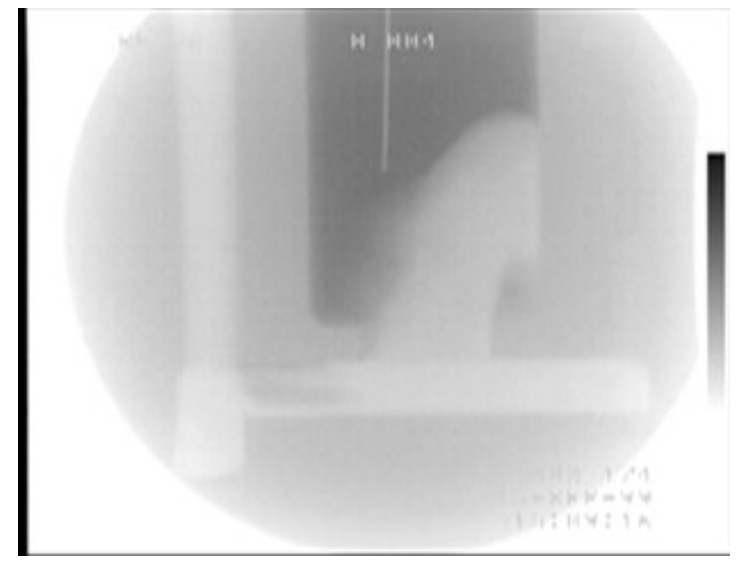

(b)

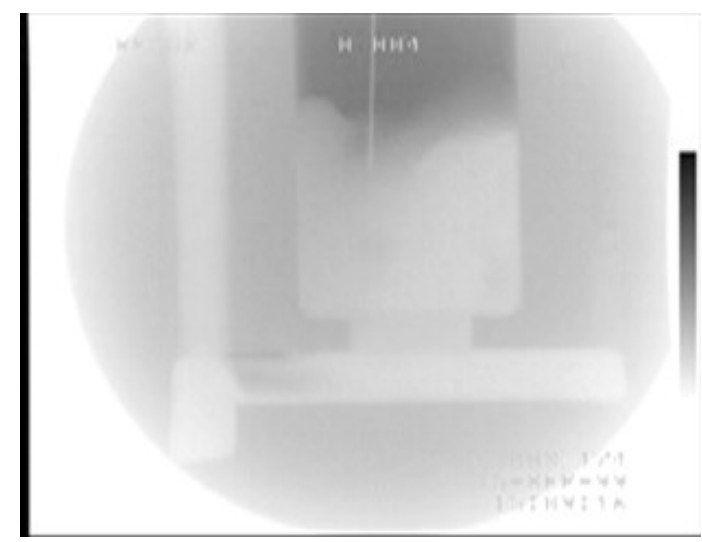

(d)

Figure 42: X-ray image sequence of metal jetting into the mold cavity of a mold with a small cross-section, squared runner extension. 


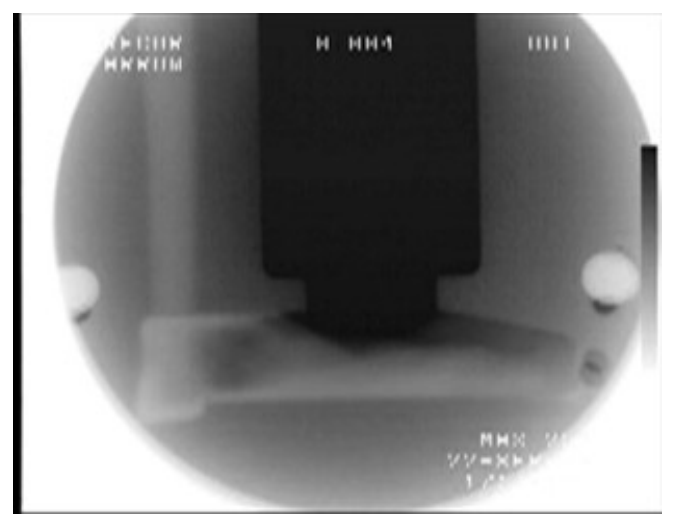

(a)

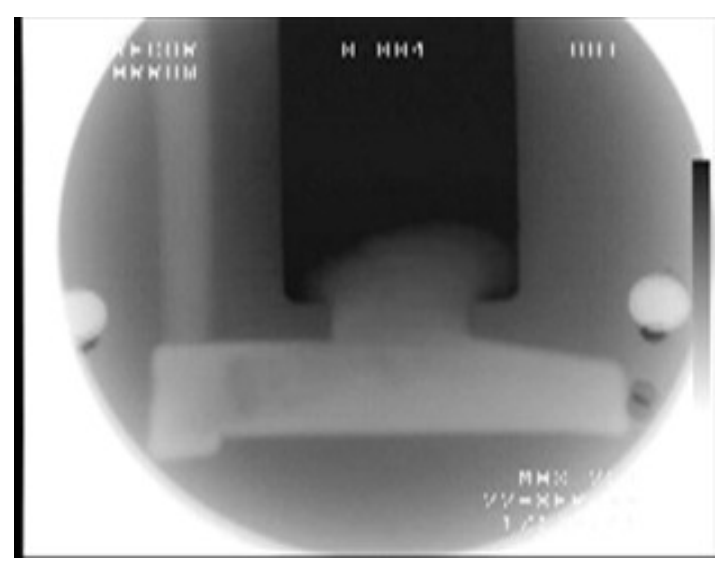

(c)

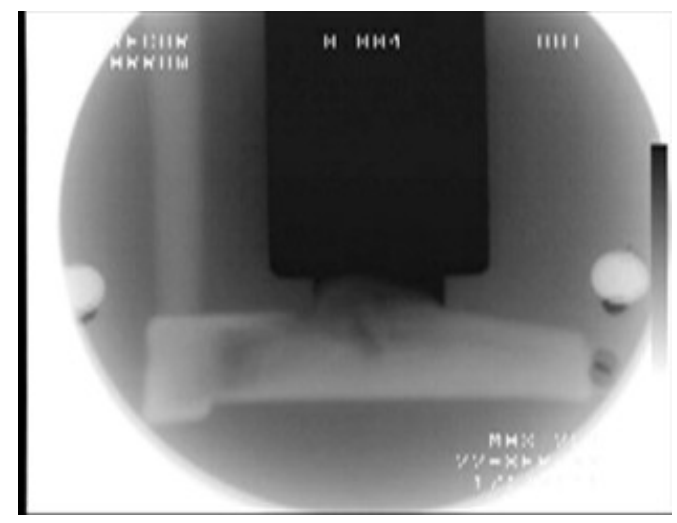

(b)

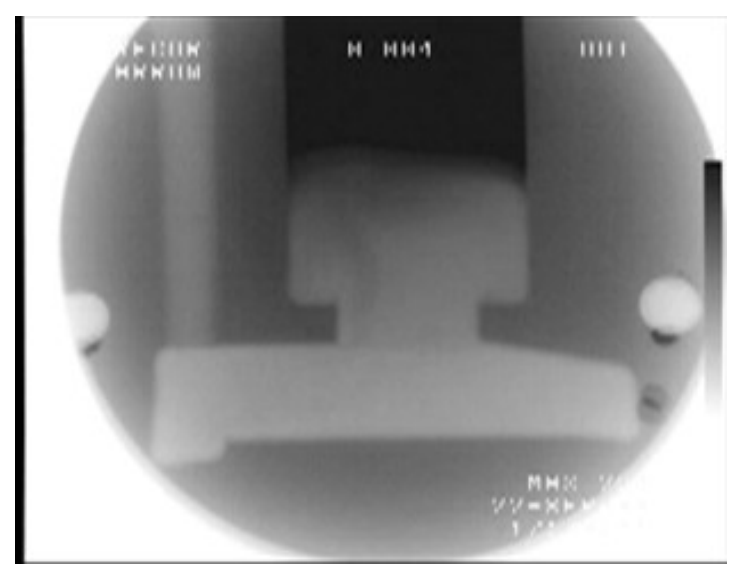

(d)

Figure 43: X-ray image sequence of metal entering the mold cavity in a mold with a larger cross-section, tapered runner extension. 


\subsubsection{Ingate}

Both ingate size and placement are important to producing high quality castings. However, because both of these are dependent on the type of gating system utilized, the behavior of ingates will be discussed in the following Gating System Behavior section.

\subsection{Gating System Behavior}

\subsubsection{Bottom-Feeding}

Bottom-feeding is the more traditional form of gating in permanent molds. In these castings, yields are relatively good and the methods for bottom-feeding are relatively well understood by designers. Some bottom-feeding designs exhibit jetting when the metal passes through the ingate into the mold cavity. This primarily happens in the squared runner extension. In the mold with a tapered, large cross-sectioned runner extension, jetting is not as prevalent.

When a filter is added to the system, much of the jetting is eliminated. The filter porosity naturally causes a drastic reduction in metal velocity into the metal cavity. A less turbulent flow results as the metal enters the mold cavity. In this mold, turbulence is observed just prior to the filter caused by a build-up of metal. However, because this is prior to the filter, this turbulence should not be detrimental to the integrity of the casting. 


\subsubsection{Top-Feeding}

Top-feeding systems do not appear to be efficient in filling mold cavities. The high drop of metal may produce oxide formation and trapped air. This is especially detrimental to the casting, since all portions of the gating system traditionally used to prevent these defects appear prior to the metal's turbulent flow. One unique observation

of the top-feeding system is that the retarding of metal flow caused by adding a filter may actually cause an increase in velocity as the mold cavity fills. The lower velocity causes a much steeper angle of entry of the metal stream into the mold cavity. Therefore, the metal drops much further before hitting the mold wall and reducing the flow rate. This causes the metal to follow the contours of the wall and climb back up the opposite side of the casting as previously shown in Figure 38. A very large turbulent circulating effect results from this large drop.

When a filter is not used, the entry angle of the metal stream to the mold cavity is very shallow and causes the metal to hit the opposite wall very soon after entering the cavity. Since the velocity is still rather high, some metal follows the contour of the mold wall, while the remaining metal actually bounces back as previously shown in Figure 39. The fact that the metal bounces off the mold wall indicates very high velocities and is, in itself, a form of turbulence that can result in oxide formation. The two metal flow patterns then meet at the bottom of the casting and react against one another, causing a rather even and gradual filling of the mold in comparison to the mold with a filter. 
Because of the relatively slow and even filling of the mold cavity, more time allows oxides and trapped air to reach the top surface of the casting. 


\subsubsection{Side-Feeding}

When properly designed and placed, a long, thin ingate placed on the side of a casting can produce very good filling patterns in a casting. Reduced turbulence was noticed in the side-feeding technique. The flow of the metal into the mold cavity appeared very laminar with an even distribution as the casting filled. Therefore, no filter was needed to slow the metal flow. In the case of the side-feeding system, the potential yield is lower because so much more metal is needed for the feeder. However, by eliminating filters, cost is reduced. Despite this decrease, a slight increase in cost of machining in incurred because the ingate follows the entire height of the casting rather than a small section on the base. With the ingate being thin, however, this cost increase should be negligible. Another possible problem can arise with the fact that one half of the casting will be hotter than the other because of the presence of hot metal in the gating on one half and just air on the other. This can lead to uneven cooling and possible shrinkage defects. 


\subsection{Special Considerations}

When analyzing the tracer simulations and comparing to the videos, the simulations tend to have a slightly higher velocity. This can be attributed to the fact that the head pressure for the simulations was estimated for a beginning head pressure for a $14 \mathrm{~cm}$ height of aluminum. However, as the stopper is removed from the aluminum basin, the height of the aluminum drops inherently as the metal fills the space previously occupied by the stopper rod. This is shown in Figure 44. When the stopper rod is removed, the molten aluminum level decreases nearly $0.5 \mathrm{~cm}$. Since the rate of stopper removal is not known and because the metal begins to flow immediately after the stopper is moved, the pressure versus time values used were estimated as a constant rate in pressure drop. With the removal of the stopper, this pressure drop would actually appear to be more hyperbolic with a slightly higher rate of decrease in pressure as the stopper rod is removed. Since the pressure is the driving variable in these simulations, the simulations result in a slightly higher velocity, since the simulation pressures are slightly higher than the actual values. 


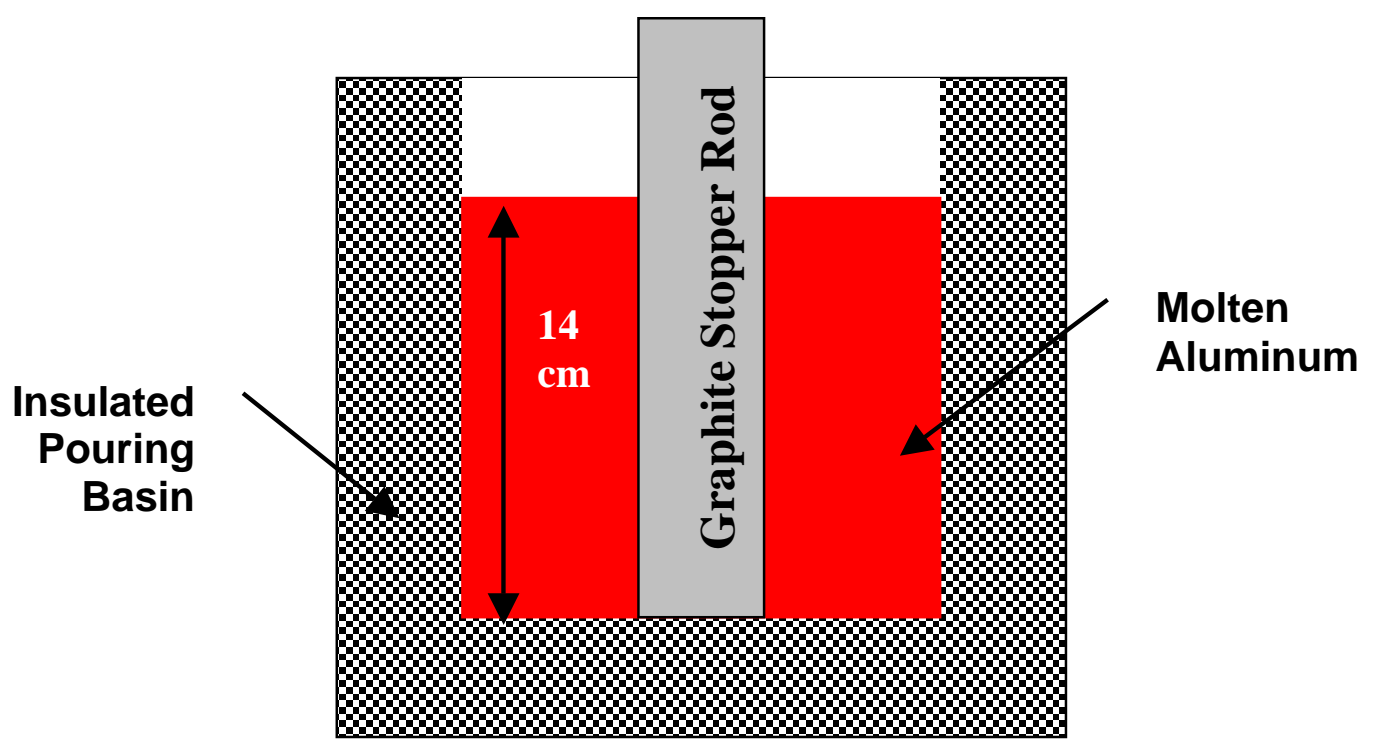

(a)

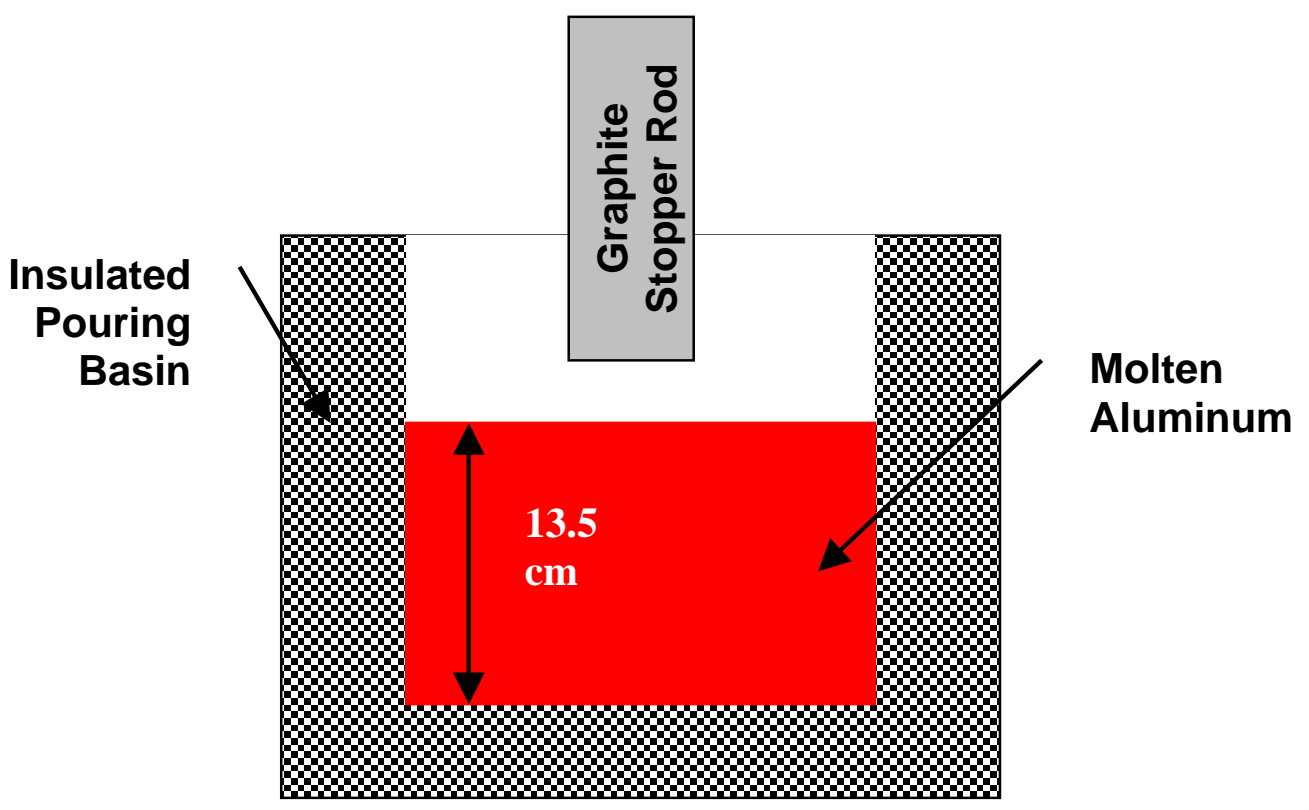

(b)

Figure 44: (a) Schematic of filled basin with stopper rod in place.

(b) Schematic of filled basin with no stopper rod (same volume of metal as in (a). 


\subsection{Evaluation of Mold Designs}

The mold designs described in this section are numbered sequentially, starting from Mold 8. These mold were designed in cooperation with the members of the AFS Permanent Mold Committee. Figure 45 shows a solid model of Mold 8. It incorporates a tapered sprue, that ends with a horn-shaped transition into a vertical riser. A thin web connects the riser to the casting. This design is similar to the "Web" gate evaluated previously, with the exception of the horn. Figures 47a-e illustrates the flow of the molten aluminum into the cavity in the presence of a 20ppi filter. The metal is initially accelerated down the sprue. In the absence of a sprue-well it encounters the circular path of the horn with a relatively high velocity. Rather than filling the horn section, the molten metal stream is pushed against the perimeter of the horn by the centrifugal forces. It then strikes the filter at an angle and bounces back into the horn. As more molten metal arrives at the filter, some bounces back and some passes through into the riser. Shown in Figure 47c are a few typical features for the flow pattern in this mold. A low-pressure pocket is visible immediately past the sprue. This pocket is prone to cause some air suction into the stream. The bounce-back from the filter is still visible but is gradually eliminated under the pressure of the incoming metal. The flow of molten metal into the cavity is dominated by the thin web. The metal cascades from riser, through the web, to the bottom of the cavity from a very low height. This feature of the web gate is very advantageous since it prevents entrapment of oxide films into the stream. It is in essence what makes this design, originally developed and evaluated by Battelle in the 50's one of the best 
gating designs for vertical molds. The other features of the Mold 8 design such as the horn are to some extent redundant and secondary in their contribution to the soundness of the casting. The computer simulation conducted in his case with Magma, provides a good prediction of the flow pattern. Mold 9 shown in Figure 48 is a simplified version of a bottom-gated system in which the runner is attached to the side of the cavity. A filter print is provided with the runner. Mold 9 was evaluated in three configurations: Mold 91 was evaluated with a 10 ppi filter (Figure 49), Mold 9-2 was evaluated with a 20 ppi filter (Figure 50) and Mold 9- 3 was evaluated without any filter (Figure 51). The results for the 10ppi and 20ppi filters are shown in Figure 52a-c. In both, the metal drops down the sprue into the sprue-well, that slows the flow velocity somewhat. The metal stream passes through the filter and flows fast past the ingate until it hits the opposite wall. It than bounces back and starts filling the cavity. The difference between the 10ppi and 20ppi filters are minimal. The joint effect of the sprue-well and the filter is to slow down the metal stream sufficiently to prevent major air suction, air bubbles and oxide film entrapment. The results for the 10ppi and without filter are shown in Figure 52d-f. In the absence of a filter, most of the metal stream shoots over the filter print. The entry velocity of the metal stream into the mold cavity is higher in this case. Even so, the casting was generally sound. It may be expected though that such a design would generate more rejects without than with filters due to excessive air and oxide entrapment (see Figure 52f). The contribution of the filter is two fold: to slow down the metal stream velocity and remove some of the oxides formed in the sprue-well. The computer simulations for these molds are shown side by side with the experiments in Figure 53a-c. Generally, the 
simulations predict well the flow patterns. Occasionally, the computer simulation tends to exaggerate the jetting of the metal. Note for instance the height of the molten metal stream as it bounces of the cavity wall in Figure 53b; it is in well in excess of the experimental observation. This aberration can be controlled by using Figure 54 shows Mold 10, an unorthodox gating design by all accounts. Members of the AFS Permanent Mold Committee decided to evaluate it anyway, because of anecdotal evidence indicating perfectly sound castings can occasionally be obtained by top pouring. Mold 10 with a 10ppi filter and w/ o filter is shown in Figures 55 and 56 respectively. The experimental observations are shown in Figure 57a- d. The metal drops to the bottom of a short sprue and from there, through a 10 ppi filter and a horizontal runner into the cavity. Because the runner is attached to the top- side of the mold cavity, most of the damage to the metal is anticipated during the fall from the runner to the bottom of the cavity. Indeed, the experimental data shows a more violent flow pattern with a vortex forming in the center of the casting. This condition is worse in the case of the mold w/o the filter as shown in Figure 57c. The metal stream jets forward and bounces off the sidewalls too, in particular because of the steps on the sidewalls, that mark the transitions between the cavity and top riser. The results of the computer simulation for Mold 10 are shown in Figures 57e- h side by side with the experimental results. Note the similarity between the predicted and the experimental vortex in Figure 57h. The table in Figure 58 summarizes the results of the evaluations conducted for Molds $8-10$ as well as the previous molds. To be noted is the fact that the ingate velocity was higher than $20 \mathrm{in} / \mathrm{sec}$ for one and only one of the mold designs: Mold 10. This is considered a critical velocity, above which the oxide film 
on the surface of the molten aluminum stream is broken and trapped in the metal. By and large, castings obtained with these gating designs were sound. The Web gating design has a very favorable flow pattern. Figure 59 shows a production permanent mold "Box Casting” with a web gate design. Figures 60-61 show the simulation results for this casting. Of particular interest is the feeding simulation shown in Figure 61e that predicts shrinkage at the bottom of the casting. Such shrinkage was occasionally observed in production at Arrow Aluminum, and measures were taken to eliminate it by adding ribs to the bottom.

\subsection{The Effect of Insulating Coatings}

It is common practice to apply an insulating coating on permanent molds. These coatings affect both the flow pattern and the heat transfer from the molten metal. In the absence of an appropriate coating, molten aluminum filling a thin section in a casting will lose heat rapidly and solidify, causing misruns and cold laps. The insulating coating therefore play an important role in extending the flow distance of the molten aluminum. Based on anecdotal evidence, the following hypotheses can be made:

\section{Advantages of Rough Coatings}

- A rough coating provides more venting by allowing air to escape.

- The air gap in a rough coating is an effective heat insulator. 


\section{Disadvantages}

- The "friction" at the mold wall is higher, thus slowing down the metal stream.

- To investigate the effect of the coating roughness, a special permanent was fabricated. This mold is depicted in Figure 63. The following procedure was used to determine the effect of insulating coating roughness:

\section{Method}

Observe flow through a thin web that simulates a thin section of a casting.

\section{Equipment}

- Open-ended vertical mold (no cavity) with web gate.

- Adjustable web inserts that allow changing the web thickness.

- Foseco coatings ESS 34 (rough) and E39 (smooth) applied to the web inserts.

\section{Experimental Procedure}

- Preliminary sensitivity study: How much does web thickness affect exit velocity? (with water and molten aluminum).

- All other parameters (head, mold temperature, metal temperature are kept constant).

- Measurement of metal velocity at the exit from the web.

\section{$\underline{\text { Results }}$}

The sensitivity study yielded an interesting result: in the presence of a riser, the web 
thickness did not have a major impact on the velocity of the jet exiting the web. Instead,of jetting more or less, the metal (or water) tends to rise higher or lower in the riser. In other words, the thin web acts as a choke. The thinner the web the more it chokes the flow and the more pressure is required to overcome the resistance to flow. The end result is that the metal level in the riser needs to be higher before it can flow past the web.

Figure 64 illustrates the flow pattern of water and molten aluminum 356 in mold 61 with a web thickness of $2.0 \mathrm{~mm}$. The velocity of the exiting jet can be determined from the distance it jets away from the mold. The jetting was videotaped for different web thicknesses. The jet velocity was measured from these videotapes and plotted as a function of web thickness as shown in Figure 65. While there is a slight drop in the exit velocity as the web becomes thicker, it is relatively a small drop. The height of the water in the riser is plotted as a function of the web thickness in Figure 66. As the web becomes thinner, the level of the water in the riser rises significantly.

Figure 67 depicts the experimental mold used to study the effect of coating roughness.

The web is created by two modular, removable inserts. These can be coated with different coatings and placed in the mold to create a web of desired thickness. Note the shorter web and riser. These are designed to eliminate the "self-regulating” mechanism described previously, whereby increased resistance to flow exerted by a thinner web led to an increase in the level of the liquid in the riser. In the short web/riser version, the exit velocity of the liquid jet is determined by the head of the metal and the frictional losses. For a given metal head and web thickness, the losses are determined solely by the 
coating. A smooth coating will generate more frictional losses because of the extensive surface contact with the molten metal. For the same reasons, a smooth coating also provides better interfacial heat transfer and thus will have a stronger chilling effect on the thin layer of metal flowing through the web. Conversely, a rough coating will generate less frictional losses because the molten metal and the oxide skin attached to it only make contact with the asperities (high points). The interfacial heat transfer is lower, because of the air trapped between the asperities of the rough coating and the metal. A rough coating will therefore be more thermally insulating, a well known fact among permanent mold casters. The roughness of the coating can be easily determined by looking at it head-on as shown in Figure 68. In this case, the roughness i.e. average distance between highest and lowest points, is ca. $38.6 \mathrm{~mm}$. Figure 69 shows the molten metal jet at 1470 degrees Farenheit as it exits a $1.6 \mathrm{~mm}$ web. The initial head is 5.5 inches. The top sequence was videotaped for a web coated with a rough coating. The bottom sequence was videotaped for a web coated with a thin coating. Note the web coated with the rough coating stayed open for 12.54 seconds while the web coated with the thin coating only stayed open for 6.27 seconds and then froze. Evidently, the rough coating would do a much better job filling a thin section of a permanent mold. These results are illustrated graphically in the plot shown in Figure 70 . Figure 71 is a repeat of the same experiment at lower metal temperature 1320 degrees Farenheit. The rough coating still keeps the web open longer, but both freeze after a shorter time of about 2.2 seconds. It should be noted this time is not only a function of temperature but also of the metal head. A higher head would create a higher hydrostatic pressure, thus keep the web open for a longer 
time. The experimental technique utilized for these experiments can provide valuable information on the interdependencies between metallostatic head, mold temperature, metal temperature and web thickness as related to filling of thin sections coated with rough and smooth coatings. 


\subsection{CONCLUSIONS}

Several conclusions can be derived from this study:

1) A sprue-well, as designed in these experiments, does not eliminate the vena contracta. Because of the swirling at the sprue-base, the circulating metal begins to push the entering metal stream toward the open runner mitigating the intended effect of the sprue-well. Improved designs of sprue-wells should be evaluated.

2) In order for a runner extension to operate efficiently, it must have a small, squared cross-section. If it is tapered, the first metal to enter the system is not effectively trapped. If the cross-section is large, there is less turbulence when aluminum enters the mold cavity in comparison to the smaller cross-sectioned, squared runner. However, a large runner reduces yield.

3) In bottom-feeding gating systems, a filter can significantly improve the filling of the casting. The filter helps to slow the metal flow rate enough to reduce jetting into the mold cavity.

4) In top-feeding gating systems, a filter can initially slow the metal flow rate, but because the metal drops after passing the filter, higher velocities are achieved during free-fall when a filter is in place.

5) Side-feeding gating systems provide less turbulent flow into the mold cavity. The flow is comparable to a bottom-feeding gating system with a filter. Using a 
properly designed side-gating system instead of a bottom-feeding system with a filter can potentially save the cost of the filter.

6) Rough coatings promote better fill than smooth coatings. This conclusion seems at first counter-intuitive. One tends to assume a rough coating creates more friction resistance to the flow of molten metal. In actuality the molten aluminum stream flows inside an oxide film envelope. When this film rests on top of the ridges of a rough coating the microscopical air pockets between the coating and the oxide film provide more thermal insulation than in a smooth coating. This insulation promotes longer feeding distances in the mold as demonstrated in the experiments. 


\section{WORKS CITED}

1. Barry, S.; “Computer Simulation,” Aluminum Permanent Mold Handbook, American Foundry Society, Chicago, 2001.

2. Buchen, W.; "The Aluminum Permanent Mold Casting Process - A Contribution to the Current State,” Transactions of the Seventh International Light Metal Congress (1981), 272-275.

3. Wukovich, N.; Metevelis, G.; “Gating: The Foundryman's Dilemma, or Fifty Years of Data and Still Asking 'How’?,” Transactions of the AFS (1990), 285-302.

4. Mar’yanskii, A.V.; "Design of Gating Systems for Aluminum Alloy Diecastings,” Soviet Castings Technology (1991), 20.

5. Strobl, S.; “Good Gating Leads to Good Castings,” Modern Casting, March 1992, 45.

6. Campbell, J.; Yang, X.; Jolly, M.; "Minimization of Surface Turbulence During Filling Using a Vortex-Flow Runner,” Aluminum Transactions (2000), 67-80.

7. Campbell, J.; Rezvani, M.; Yang, X.; "Effect of Ingate Design on Strength and Reliability of Al Castings," Transactions of the American Foundrymen's Society (1999), 181-188.

8. Campbell, J.; Castings, Butterworth Heinemann, Boston, 2000.

9. Casting Defects Handbook, American Foundry Society, Des Plaines, 2000.

10. Askeland, D.; Hold, M.L.; “Kiss Gating of Aluminum Alloy Castings,” Transactions of the AFS (1975), 91-98.

11. Kotzin, E.L.; Metalcasting and Molding Processes, American Foundrymen’s Society, Des Plaines, 1981.

12. Tiedje, N.; "Flow Through Bends in Gating Systems in Vertically Parted Molds," Transactions of the AFS (1999), 581-590.

13. Webster, P.D.; "Study of the Flow of Metals in Runners," British Foundryman, 60 (1967) 314-319.

14. Apelian, D.; Mutharasan, R.; “Filtration: A Melt Refining Method,” Journal of Metals, September, 1980, 14-19.

15. Ravi, B.; “Intelligent Design of Gating Channels for Casting,” Materials Science and 
Technology, September 1997, 785-790.

16. Maeda, Y.; Kato, E.; "Effect of Gate Size on Cavity Filling and Casting Property for Aluminum Alloys,” Solidification Processing, July 1997, 70-73.

17. Halmshaw, R.; Non-destructive testing, Edward Arnold, Baltimore, 1987, 16-101.

18. Cartz, L.; Nondestructive testing: Radiography, Ultrasonics, Liquid Penetrant, Magnetic Particle, Eddy Current, ASM International, 1995.

19. Garrett, D.A.; Bracher, D.A.; Real Time Radiologic Imaging, ASTM-SPT-716, ASTM, Philadelphia, 1980.

20. Poore, L.; Real-Time Radiography Course Booklet, National Science Foundation, 2001.

21. Nariman, R.; “Gating Design Via Computer Fluid Flow Modeling,” Modern Casting, June 1998, 35-38.

22. Ono, T.; Ohtsuka, Y.; "Computer Simulation of Flow and Solidification in Gravity Die Castings,” Proceedings of the Sixteenth International Die Cast Congress and Expo (1991), 321-327.

23. Carey, G.F.; Oden, J.T.; Finite Elements: Fluid Mechanics, Prentice Hall, Englewood Cliffs, 1986.

24. Cuvelier, C.; Segal, A.; van Steenhoven, A.A.; Finite Element Methods and NavierStokes Equations, D. Reidel Publishing Company, Boston, 1986.

25. Desai, C.S.; Elementary Finite Element Method, Prentice-Hall, Englewood Cliffs, 1979.

26. Taylor, C.; Hughes, T.G.; Morgan, K.; “A Finite Element Model of One and Two Equation Models of Turbulent Flow," Recent Advances in Numerical Methods in Fluids, Swansea, Pineridge, 1980, 311-334.

27. Midea, A.C.; Schmidt, D.; “1999 Casting Simulation Software Survey,” Modern Casting, May 1999, 47-51.

28. Mollard, F.R.; Davidson, N.; "Ceramic Foam: A Unique Method of Filtering Aluminum Castings,” AFS Transactions, 78 (1970) 479-486. 


\section{BIBLIOGRAPHY}

Apelian, D.; Mutharasan, R.; “Filtration: A Melt Refining Method,” Journal of Metals, September, 1980, 14-19.

Askeland, D.; Hold, M.L.; “Kiss Gating of Aluminum Alloy Castings,” Transactions of the AFS (1975), 91-98.

Barry, S.; “Computer Simulation,” Aluminum Permanent Mold Handbook, American Foundry Society, Chicago, 2001.

Buchen, W.; “The Aluminum Permanent Mold Casting Process - A Contribution to the Current State,” Transactions of the Seventh International Light Metal Congress (1981), 272-275.

Campbell, J.; Castings, Butterworth Heinemann, Boston, 2000.

Campbell, J.; Yang, X.; Jolly, M.; "Minimization of Surface Turbulence During Filling Using a Vortex-Flow Runner,” Aluminum Transactions (2000), 67-80.

Campbell, J.; Rezvani, M.; Yang, X.; "Effect of Ingate Design on Strength and Reliability of Al Castings," Transactions of the American Foundrymen's Society (1999), 181-188.

Carey, G.F.; Oden, J.T.; Finite Elements: Fluid Mechanics, Prentice Hall, Englewood Cliffs, 1986.

Cartz, L.; Nondestructive testing: Radiography, Ultrasonics, Liquid Penetrant, Magnetic Particle, Eddy Current, ASM International, 1995.

Casting Defects Handbook, American Foundry Society, Des Plaines, 2000.

Cuvelier, C.; Segal, A.; van Steenhoven, A.A.; Finite Element Methods and NavierStokes Equations, D. Reidel Publishing Company, Boston, 1986.

Desai, C.S.; Elementary Finite Element Method, Prentice-Hall, Englewood Cliffs, 1979.

Garrett, D.A.; Bracher, D.A.; Real Time Radiologic Imaging, ASTM-SPT-716, ASTM, Philadelphia, 1980.

Halmshaw, R.; Non-destructive testing, Edward Arnold, Baltimore, 1987, 16-101.

Kotzin, E.L.; Metalcasting and Molding Processes, American Foundrymen’s Society, Des Plaines, 1981. 
Maeda, Y.; Kato, E.; "Effect of Gate Size on Cavity Filling and Casting Property for Aluminum Alloys,” Solidification Processing, July 1997, 70-73.

Mar’yanskii, A.V.; “Design of Gating Systems for Aluminum Alloy Diecastings,” Soviet Castings Technology (1991), 20.

Midea, A.C.; Schmidt, D.; “1999 Casting Simulation Software Survey,” Modern Casting, May 1999, 47-51.

Mollard, F.R.; Davidson, N.; "Ceramic Foam: A Unique Method of Filtering Aluminum Castings,” AFS Transactions, 78 (1970) 479-486.

Nariman, R.; “Gating Design Via Computer Fluid Flow Modeling,” Modern Casting, June 1998, 35-38.

Ono, T.; Ohtsuka, Y.; “Computer Simulation of Flow and Solidification in Gravity Die Castings,” Proceedings of the Sixteenth International Die Cast Congress and Expo (1991), 321-327.

Poore, L.; Real-Time Radiography Course Booklet, National Science Foundation, 2001.

Ravi, B.; “Intelligent Design of Gating Channels for Casting,” Materials Science and Technology, September 1997, 785-790.

Strobl, S.; “Good Gating Leads to Good Castings,” Modern Casting, March 1992, 45.

Taylor, C.; Hughes, T.G.; Morgan, K.; “A Finite Element Model of One and Two Equation Models of Turbulent Flow," Recent Advances in Numerical Methods in Fluids, Swansea, Pineridge, 1980, 311-334.

Tiedje, N.; "Flow Through Bends in Gating Systems in Vertically Parted Molds," Transactions of the AFS (1999), 581-590.

Webster, P.D.; "Study of the Flow of Metals in Runners,” British Foundryman, 60 (1967) 314-319.

Wukovich, N.; Metevelis, G.; “Gating: The Foundryman’s Dilemma, or Fifty Years of Data and Still Asking 'How’?,” Transactions of the AFS (1990), 285-302. 
Appendix A: Figures for Sections 3.4, 3.5 


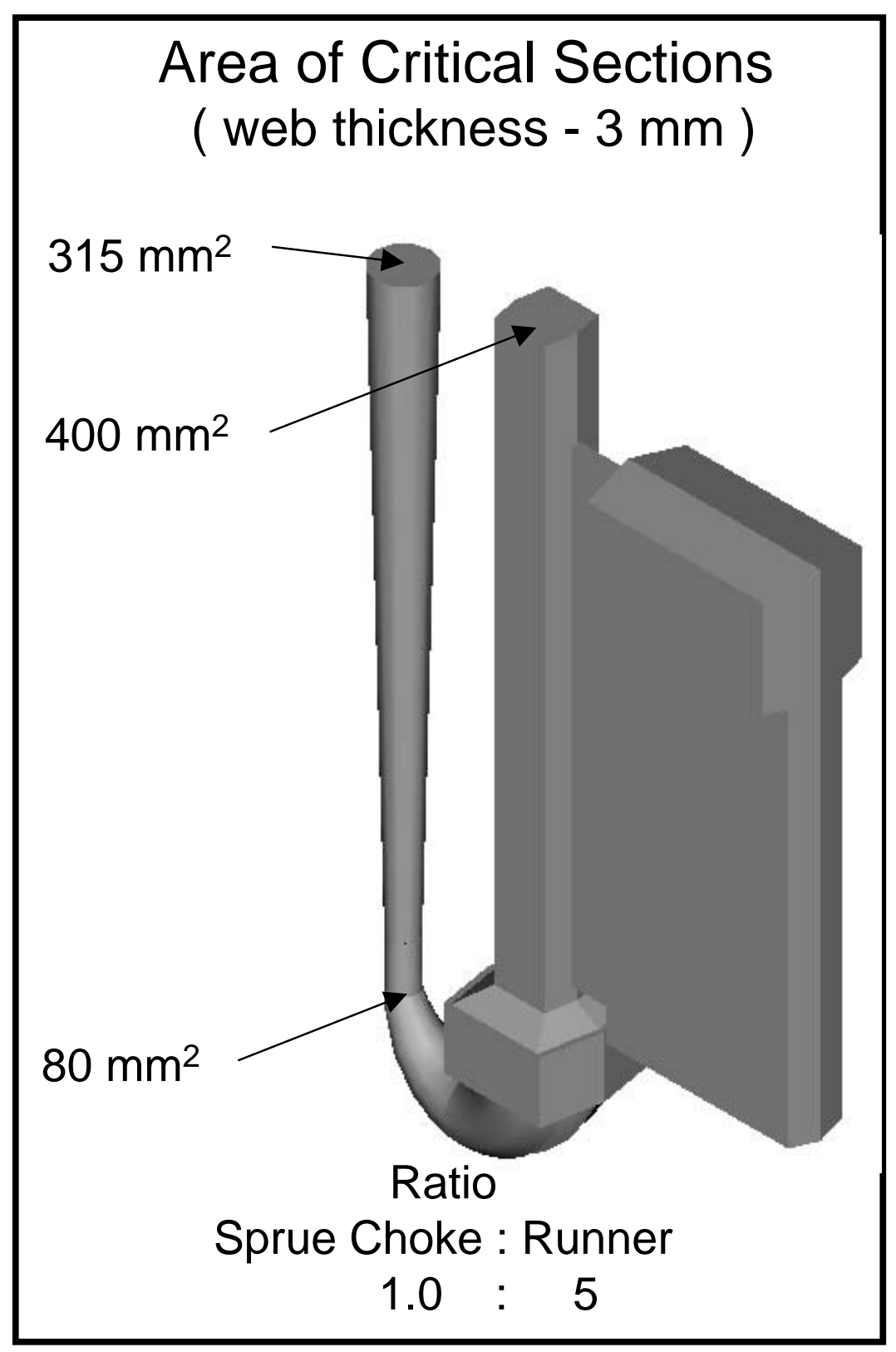

\section{Figure 45 MOLD 8- Horn Gate}

- Web gating system with a large vertical riser and a thin web.

- Horn shape runner with a horizontal 10 ppi ceramic foam filter in the runner.

- Metal flows into the vertical riser first before cascading into the mold cavity. 
Figure 46: Mold 8 (with 10 ppi Filter)- Movie (click to view)

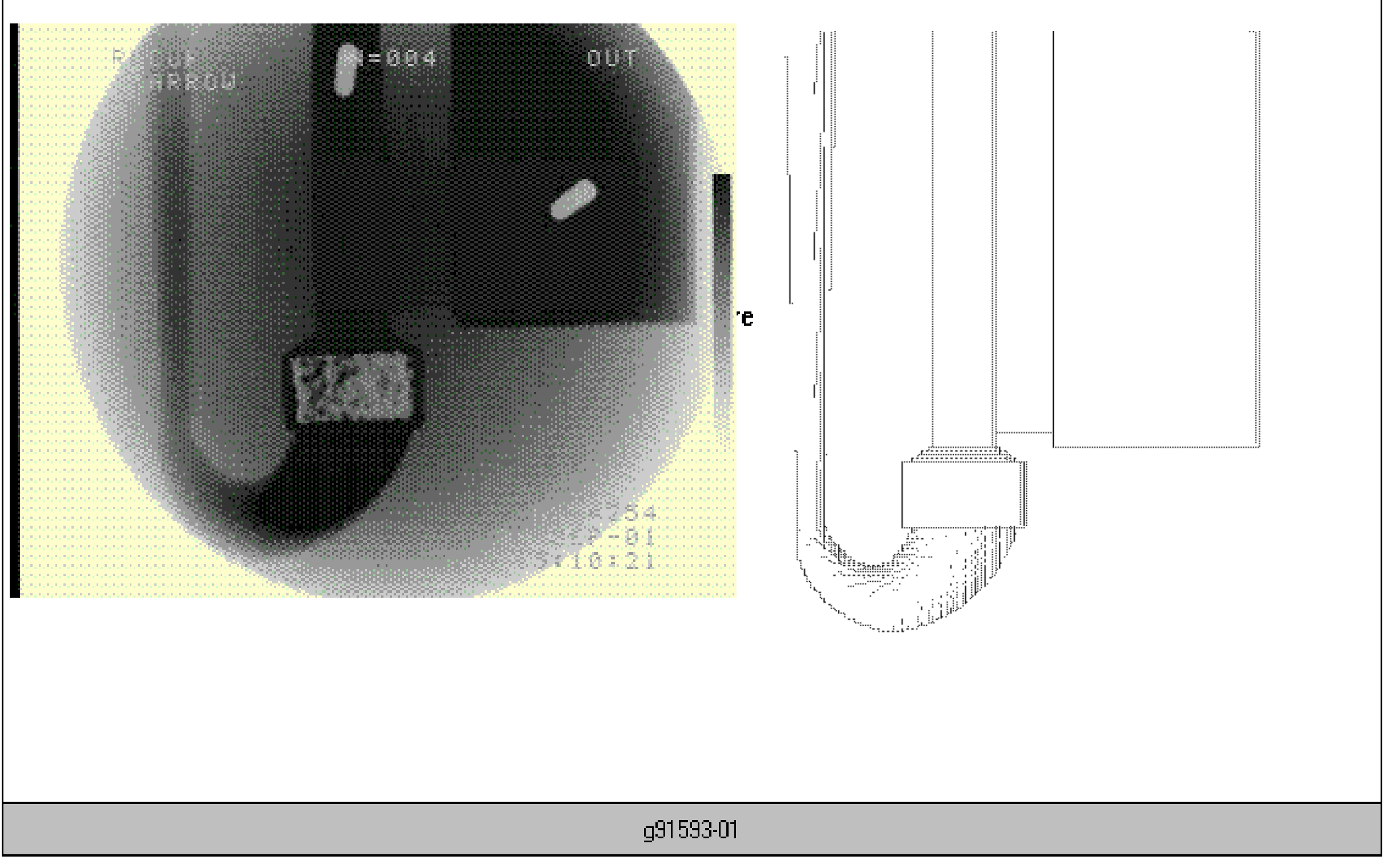


Figure 47a:Comparison of Simulation Results to Experiment In Mold 8

$$
\text { X-ray }
$$

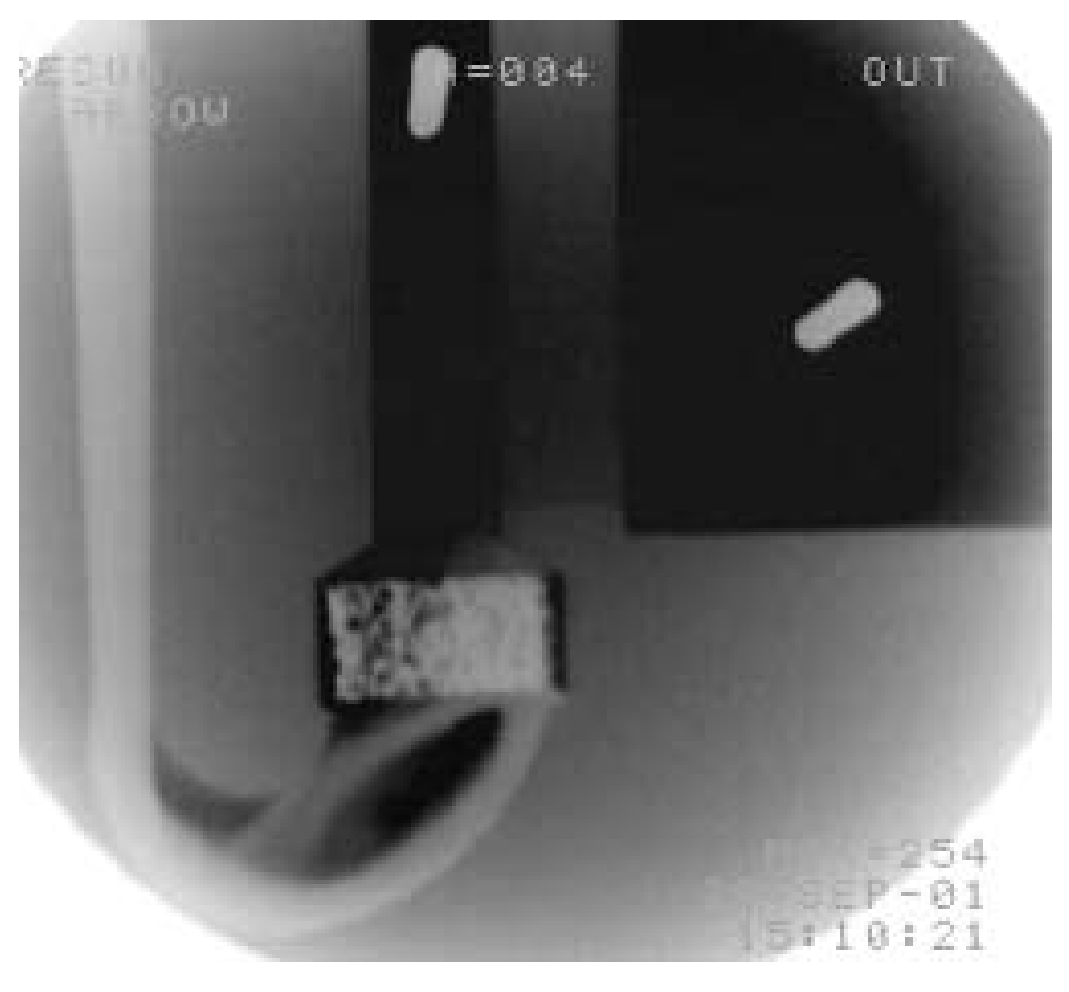

$0.20 \mathrm{sec}$

\author{
Simulation
}

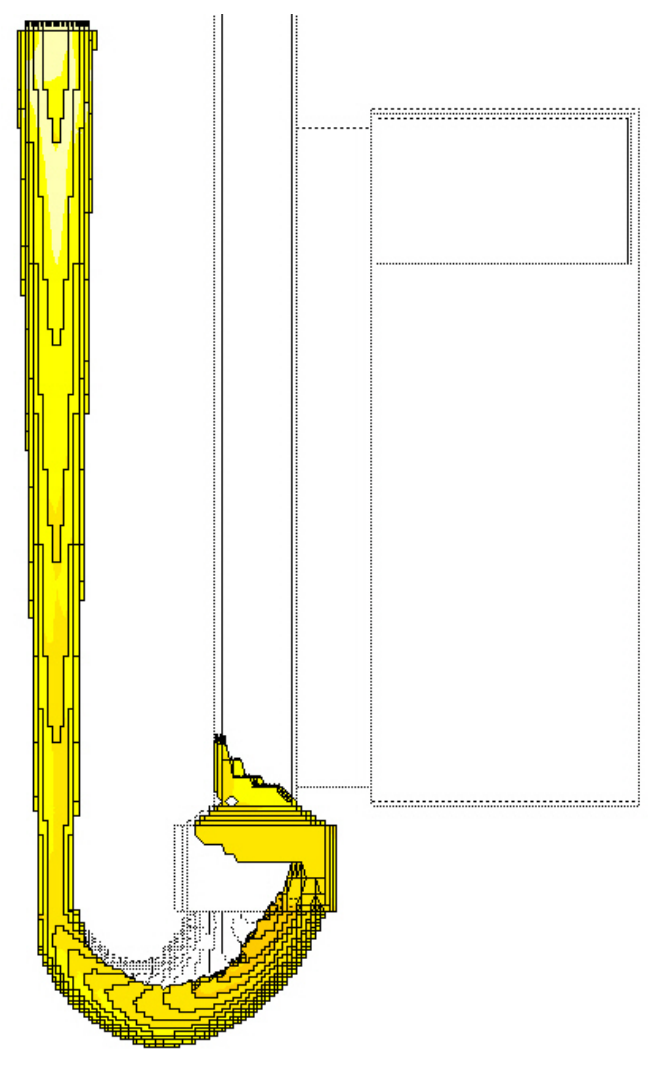

$0.25 \mathrm{sec}$ 
Figure 47b: Comparison of Simulation Results to Experiment In Mold 8

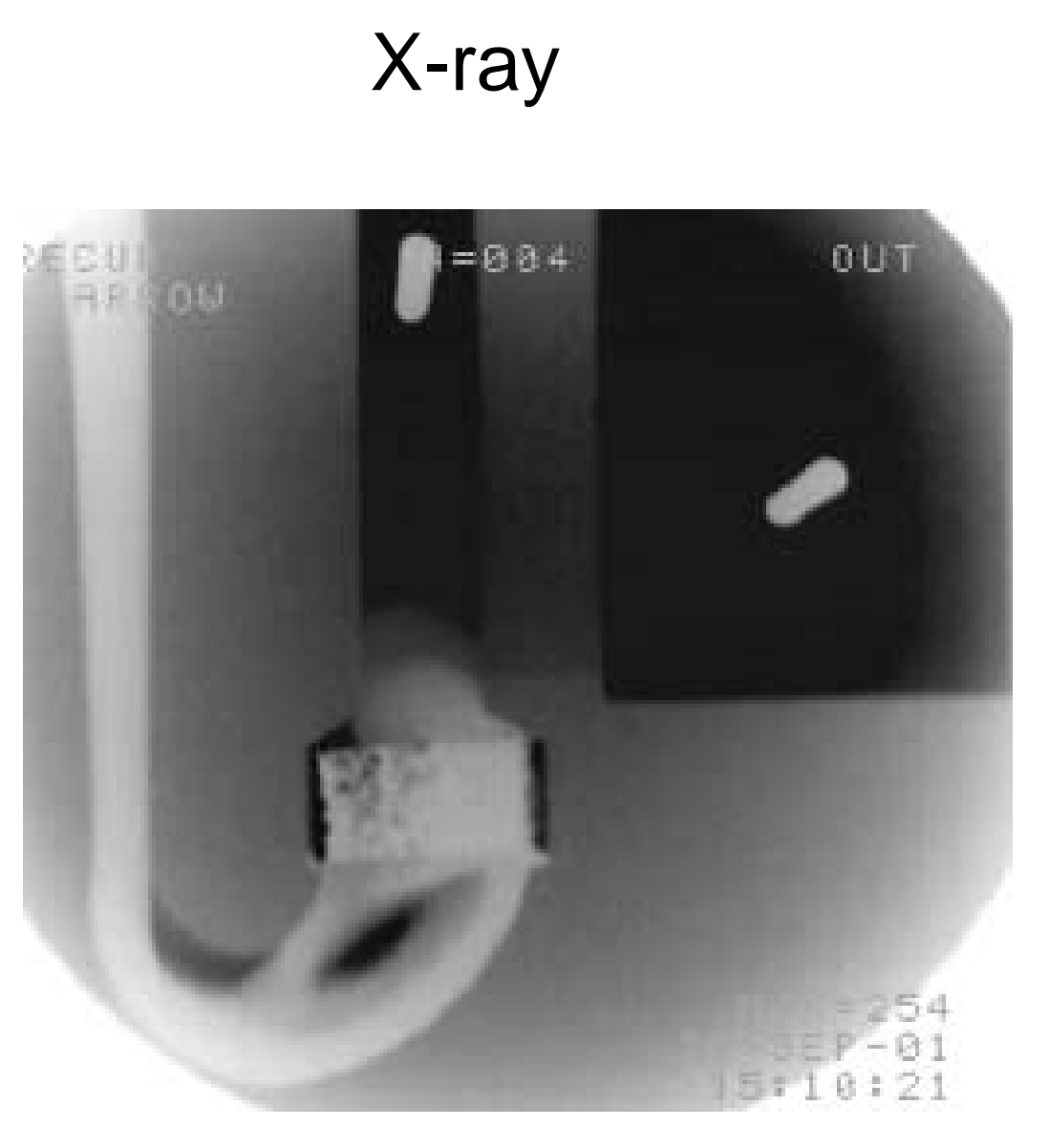

$0.36 \mathrm{sec}$

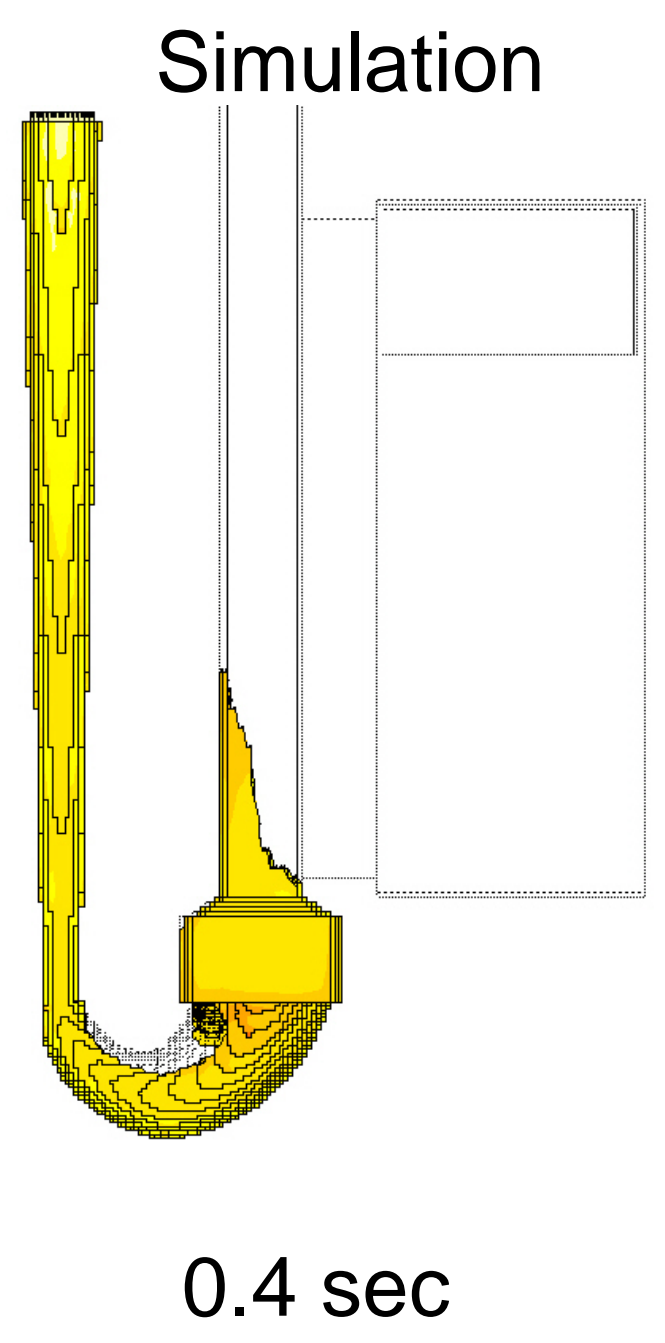


Figure 47c: Comparison of Simulation Results to Experiment In Mold 8

$$
\text { X-ray }
$$

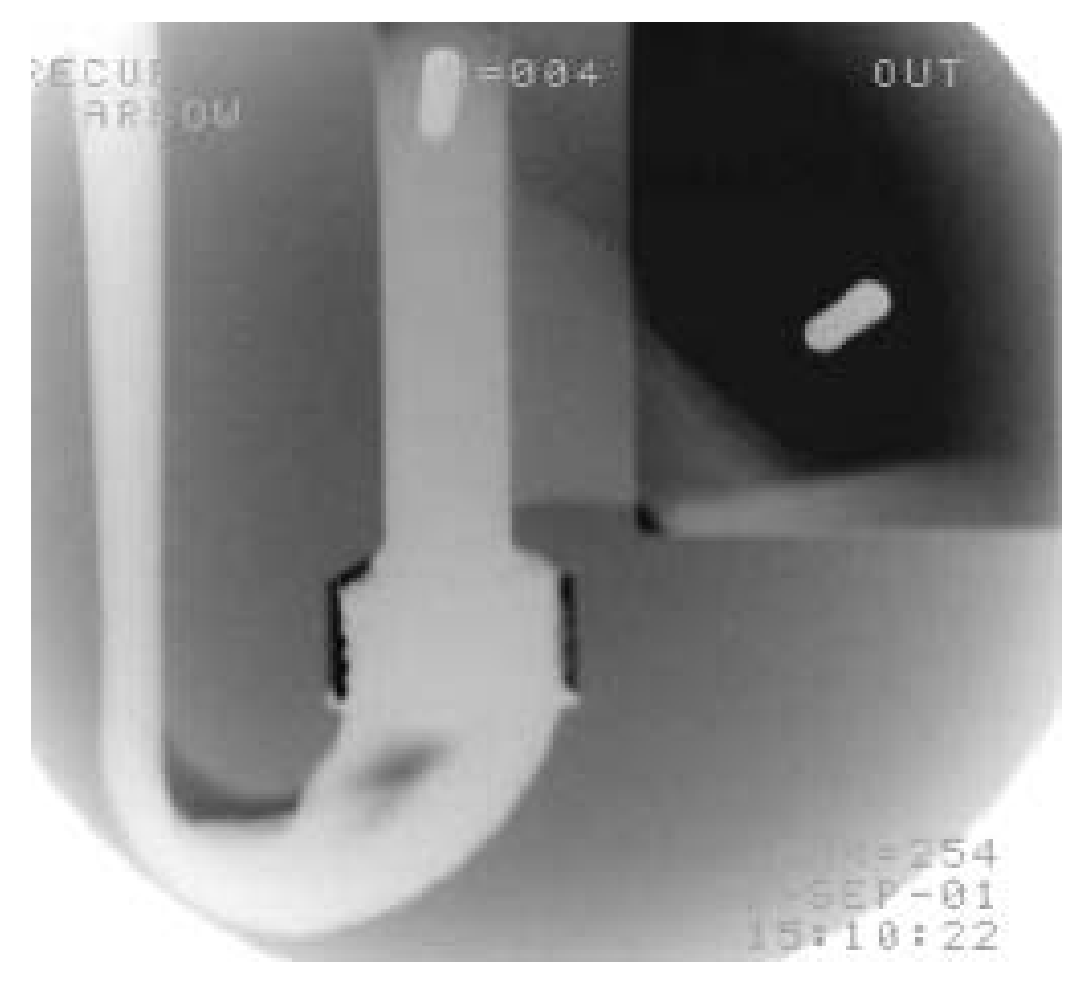

$0.60 \mathrm{sec}$

\section{Simulation}

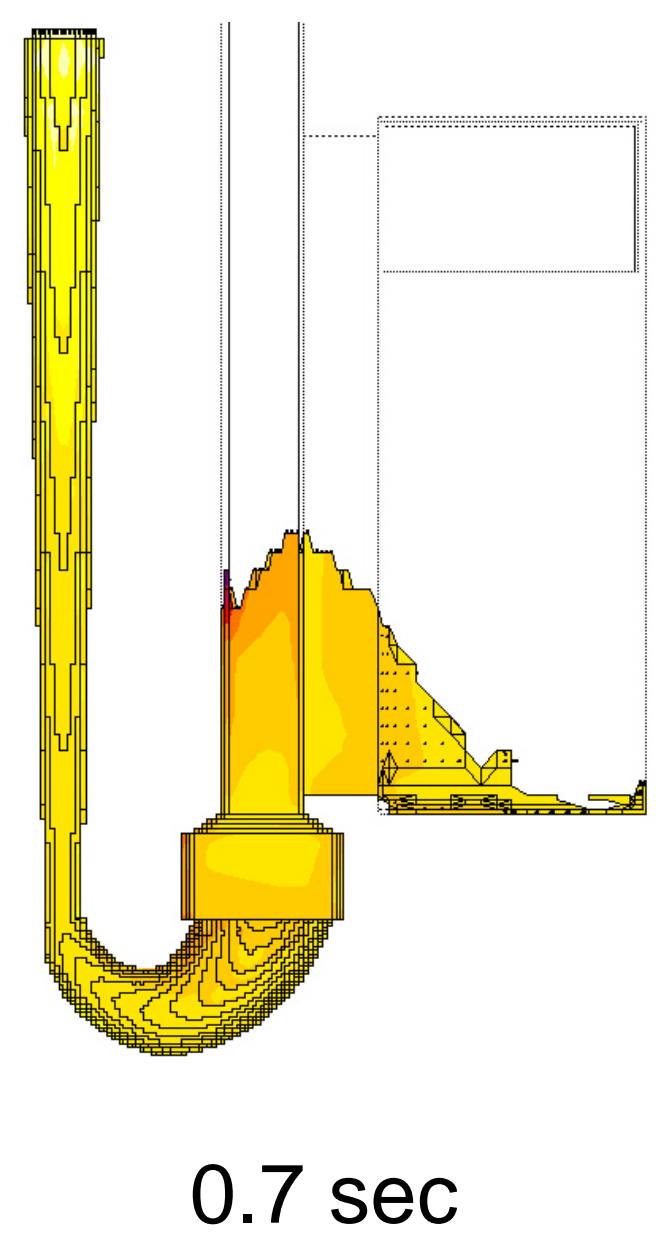


Figure 47d: Comparison of Simulation Results to Experiment In Mold 8

$$
\text { X-ray }
$$

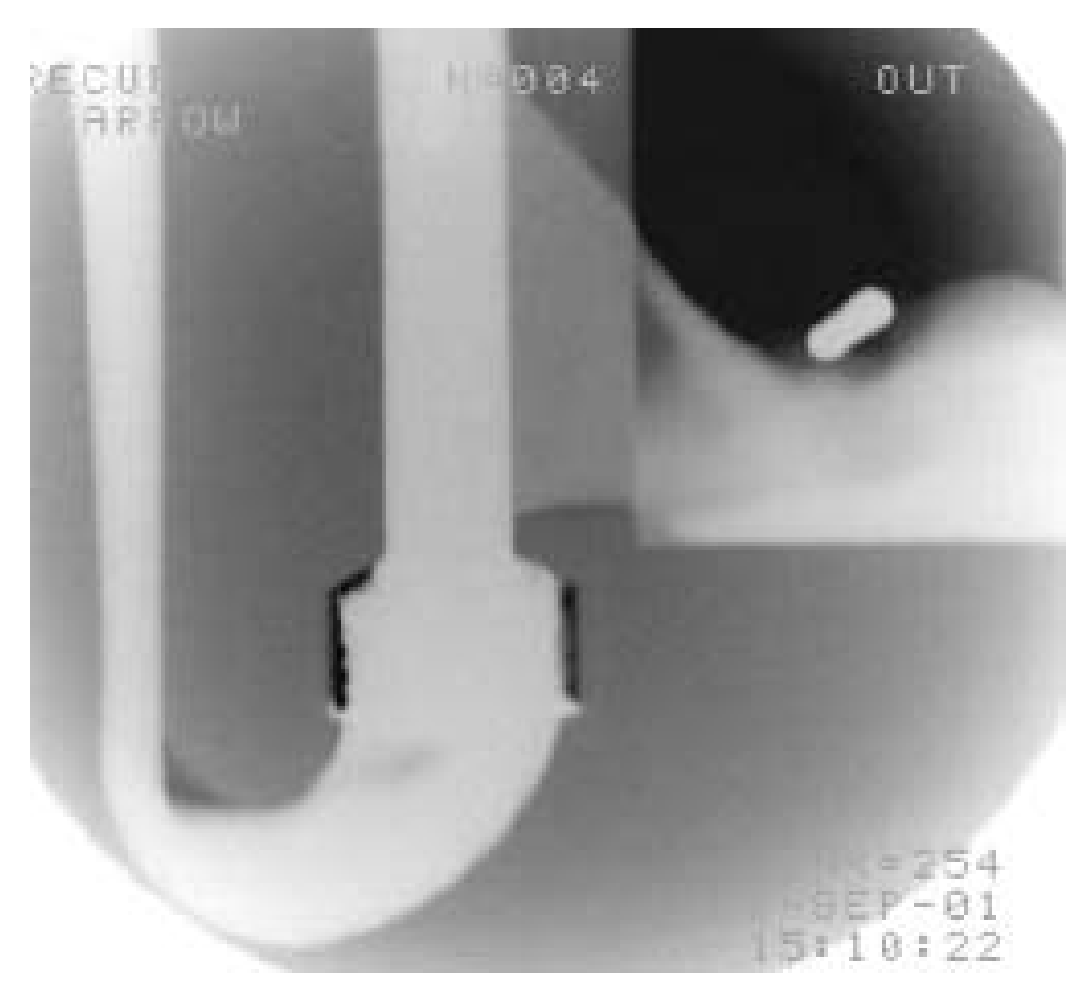

$0.9 \mathrm{sec}$

\author{
Simulation
}

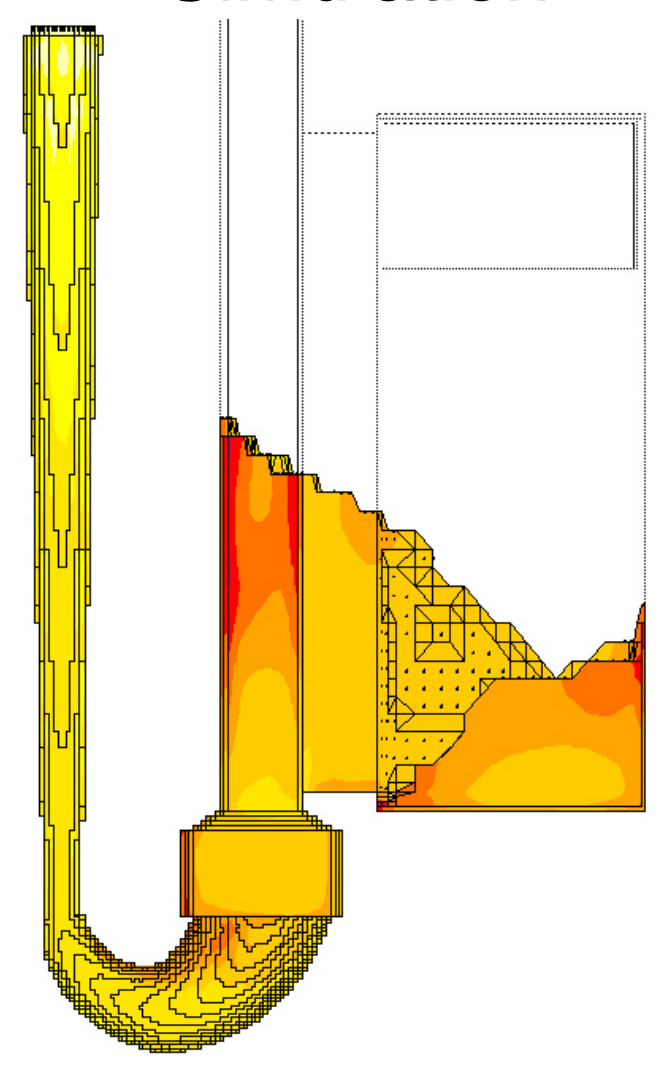

$0.8 \mathrm{sec}$ 
Figure 47e: Comparison of Simulation Results to Experiment In Mold 8

X-ray

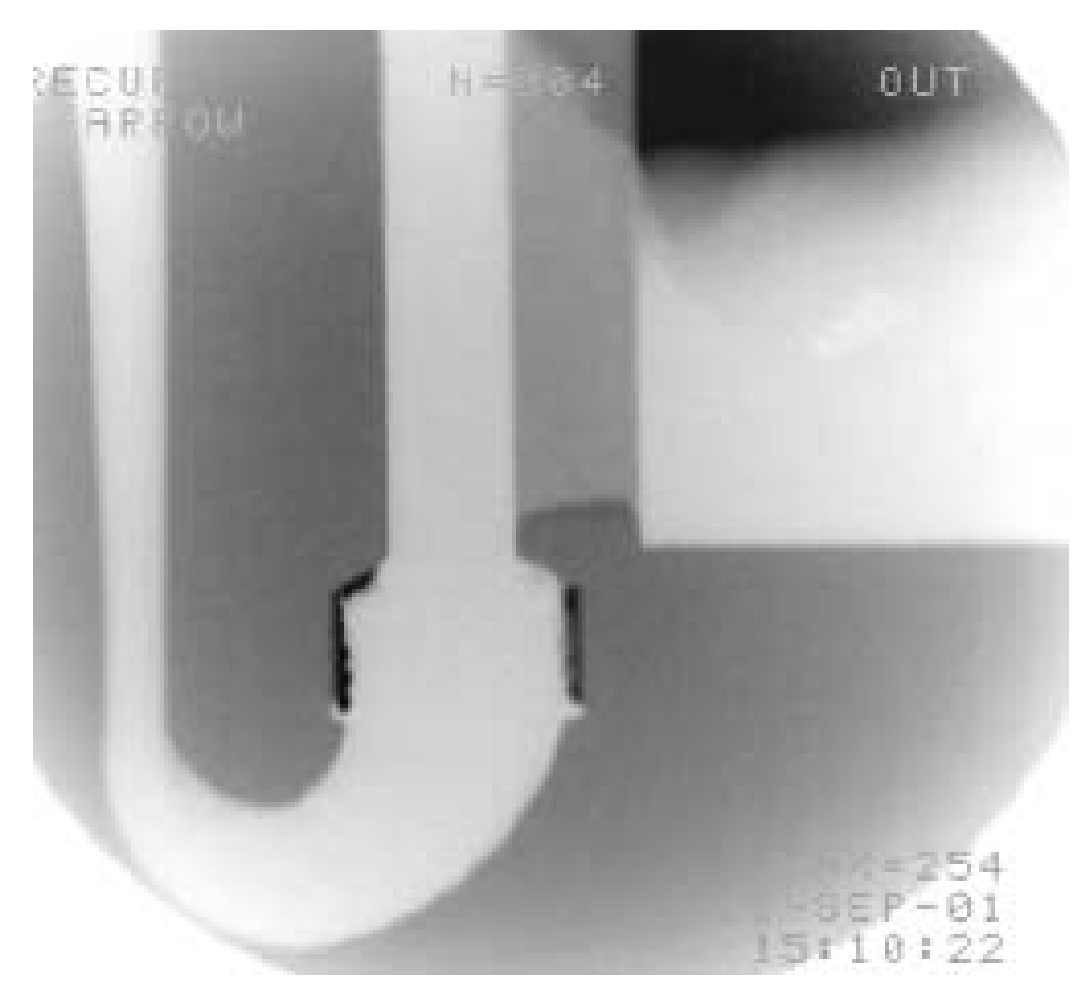

$1.4 \mathrm{sec}$
Simulation

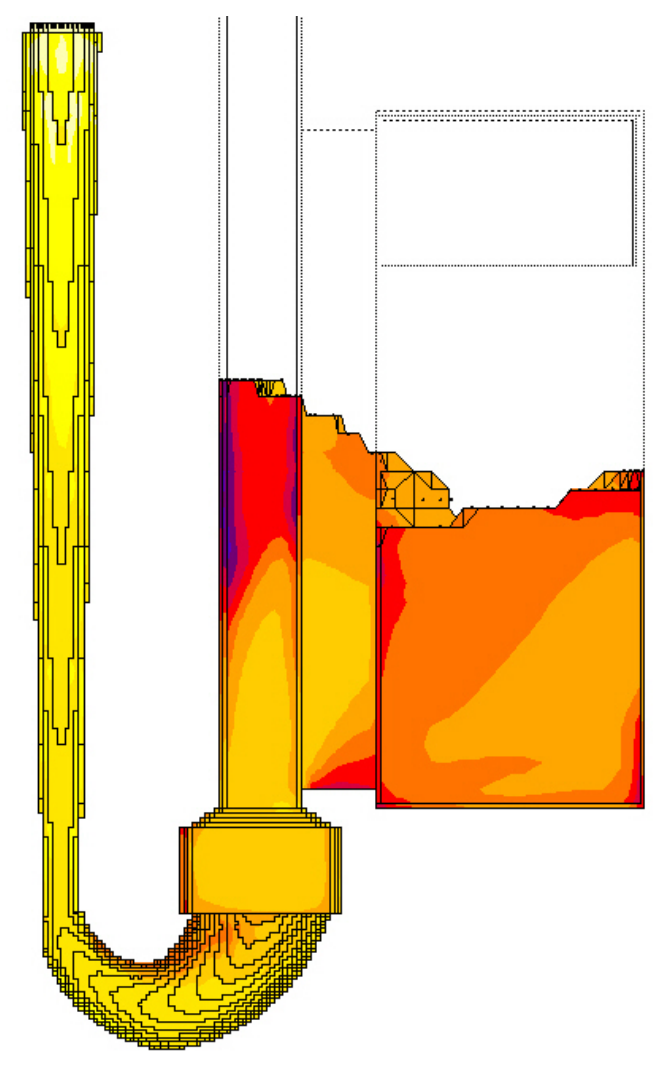

$1.3 \mathrm{sec}$ 


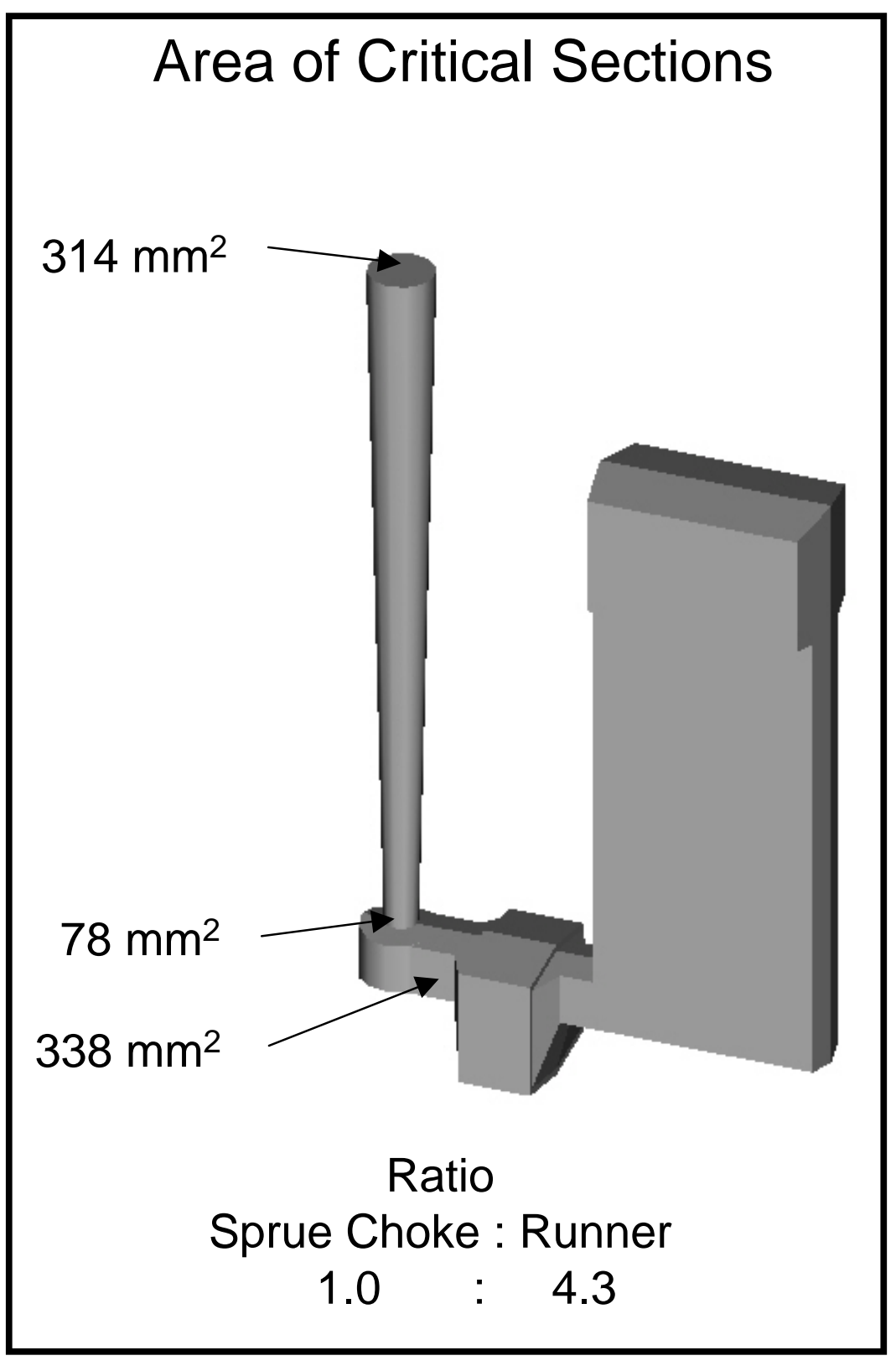

\section{Figure 48 \\ MOLD 9- Bottom-side Gate}

- Side bottom gating system with a vertical 10 ppi ceramic foam filter in the runner.

- The ppi of the filter has little effect on the flow pattern.

- Filter chokes the flow; the positive pressure prevents air entrapment. 
Figure 49: CWRU Mold 9-1 (with 10 ppi Filter)- Movie (click to view)

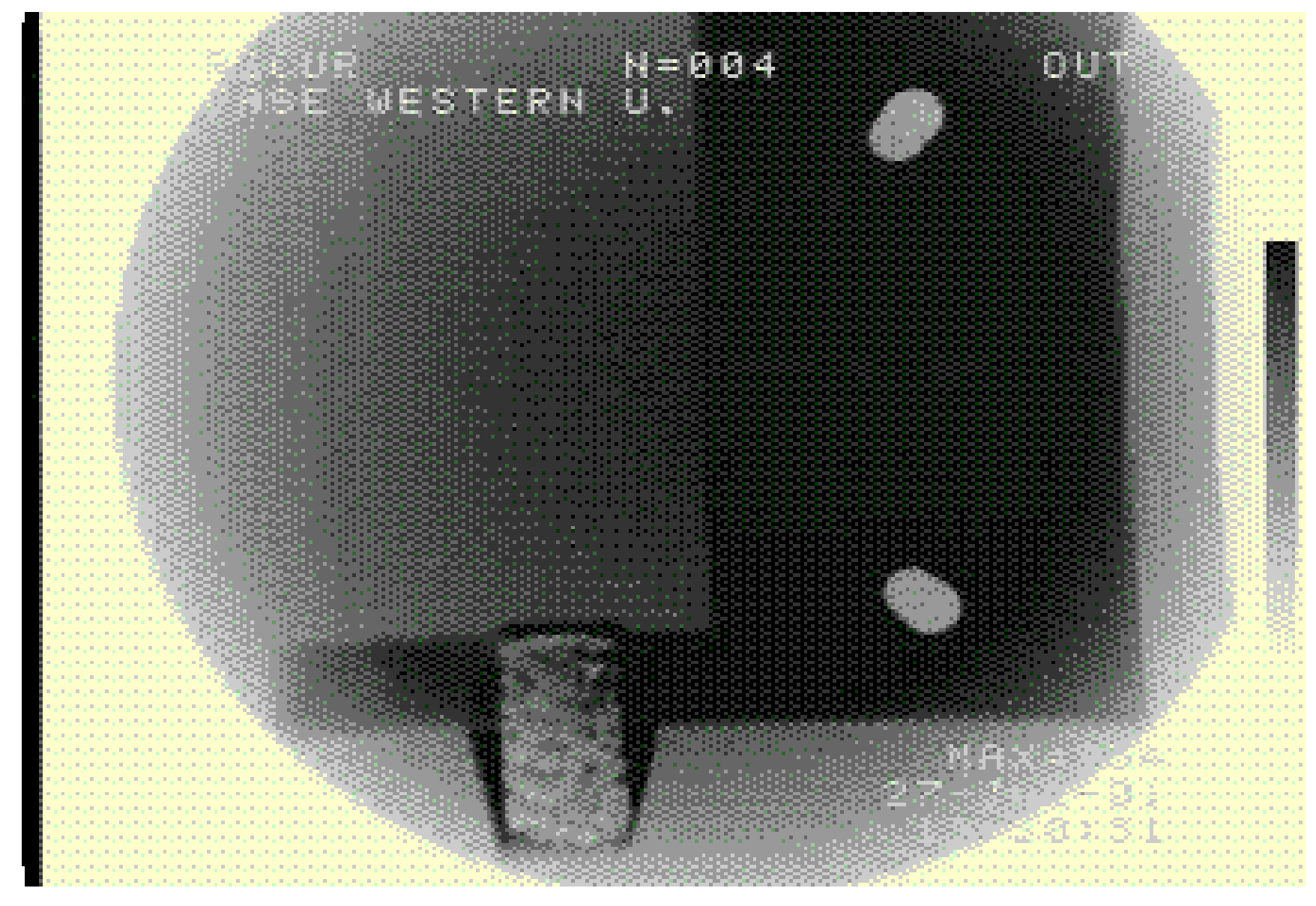


Figure 50: CWRU Mold 9-2 (with 20 ppi Filter)- Movie (click to view)

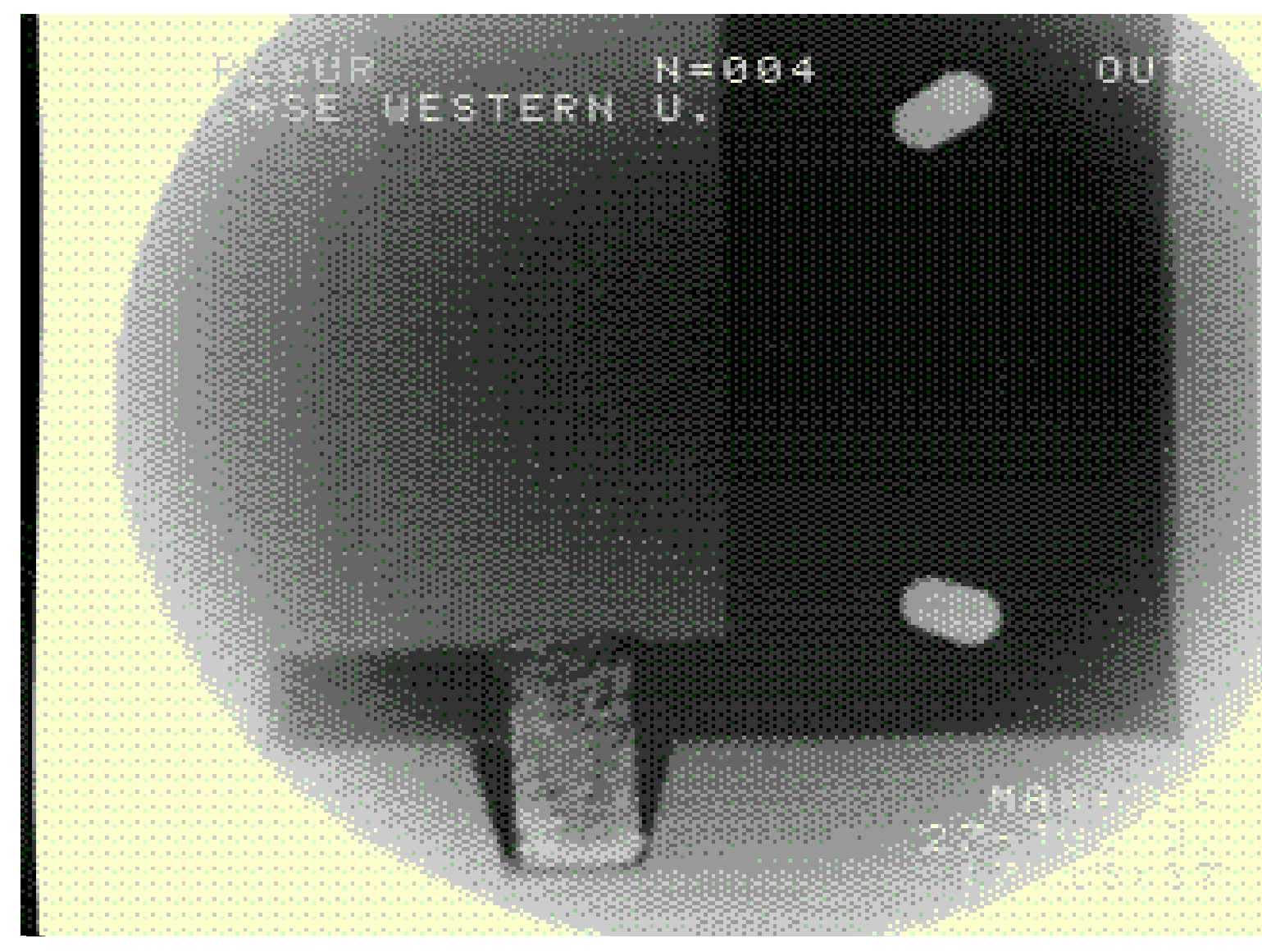


Figure 51: CWRU Mold 9-3 (without Filter)- Movie (click to view)

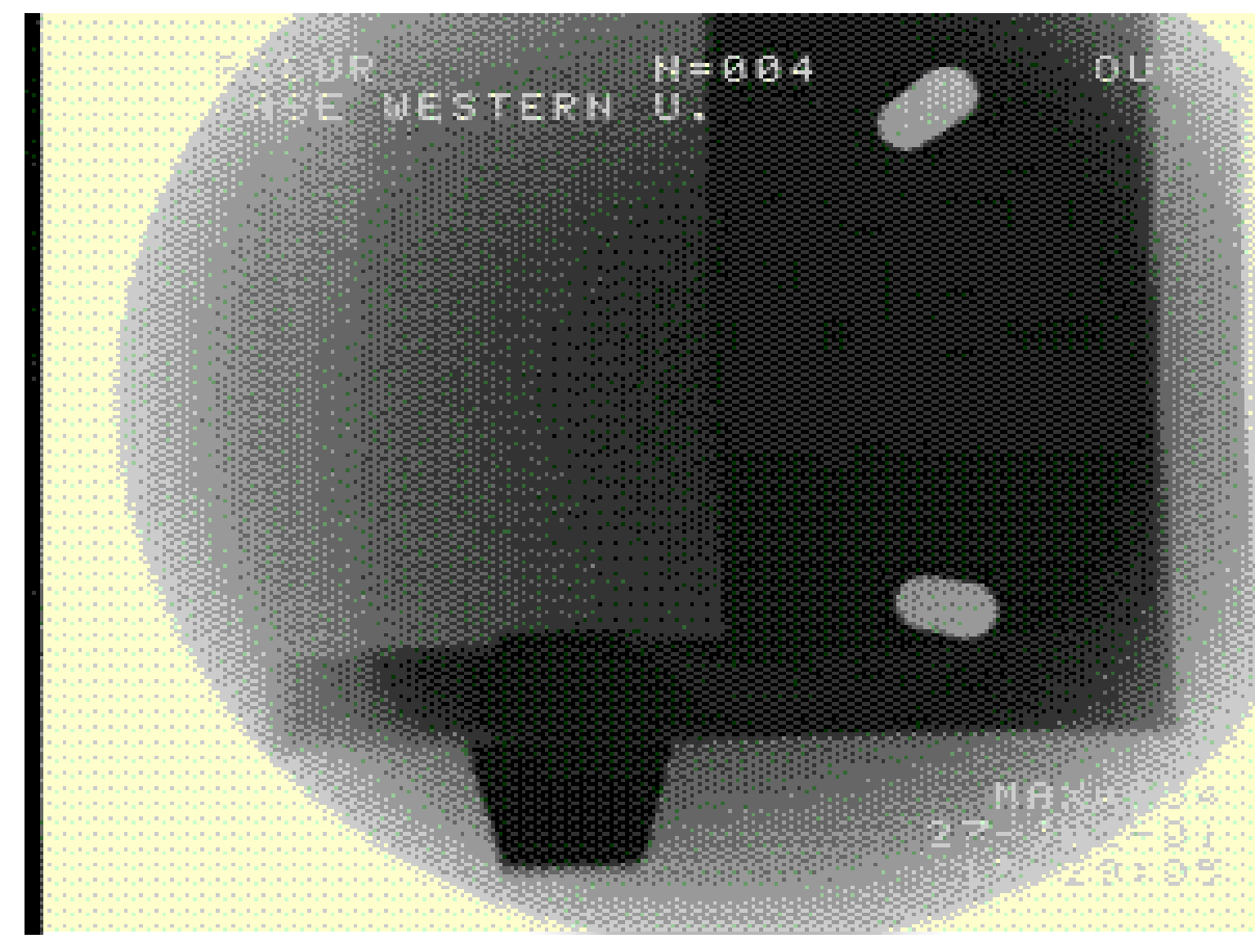


Figure 52a: Real-Time X-ray in Mold 9

10 ppi Filter

20 ppi Filter

WES TERH U. $\mathrm{U}=0.4$

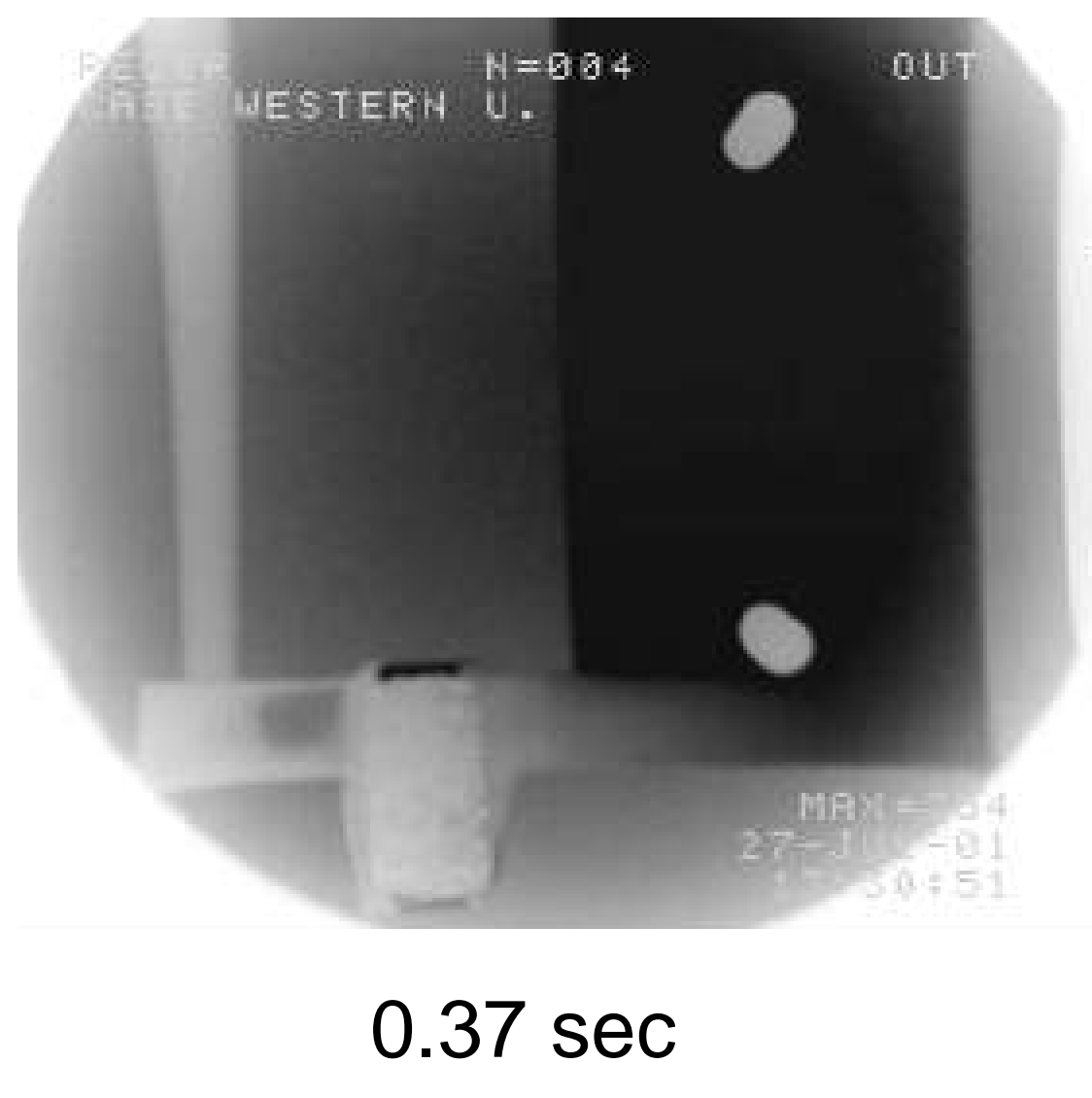

$0.37 \mathrm{sec}$ 
Figure 52b: Real-Time X-ray in Mold 9

10 ppi Filter

20 ppi Filter
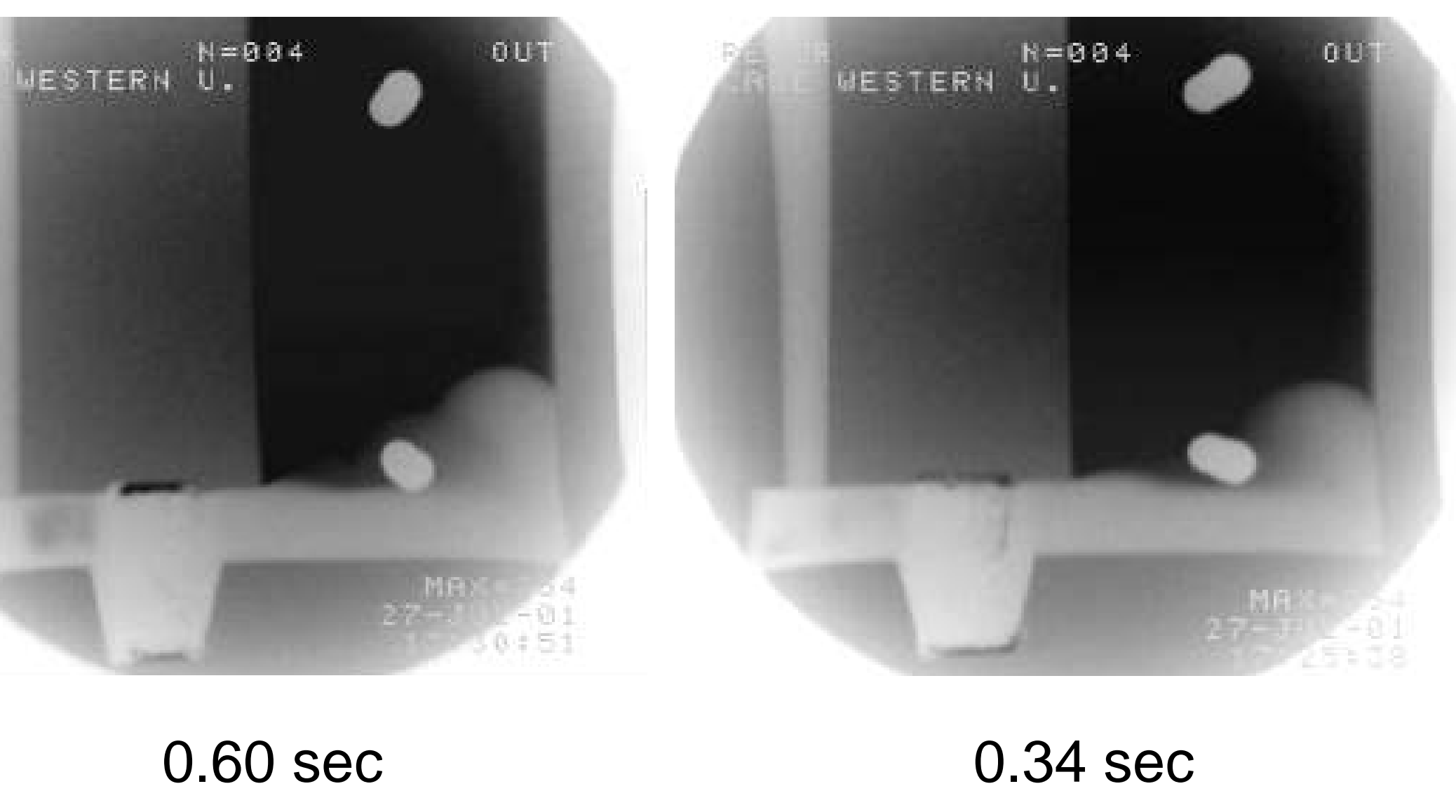
Figure 52c: Real-Time X-ray in Mold 9

10 ppi Filter

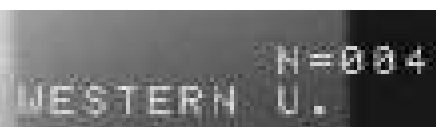

20 ppi Filter

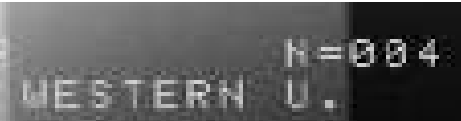

MESTERN $\mathrm{i}=0.04$

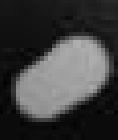

ou:

$0.83 \mathrm{sec}$

$0.83 \mathrm{sec}$ 
Figure 52d: Real-Time X-ray in Mold 9

10 ppi Filter

WESTERH U.

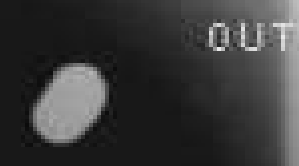

ma
Without Filter

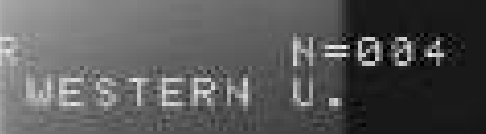

$0.37 \mathrm{sec}$

$0.27 \mathrm{sec}$ 
Figure 52e: Real-Time X-ray in Mold 9

10 ppi Filter
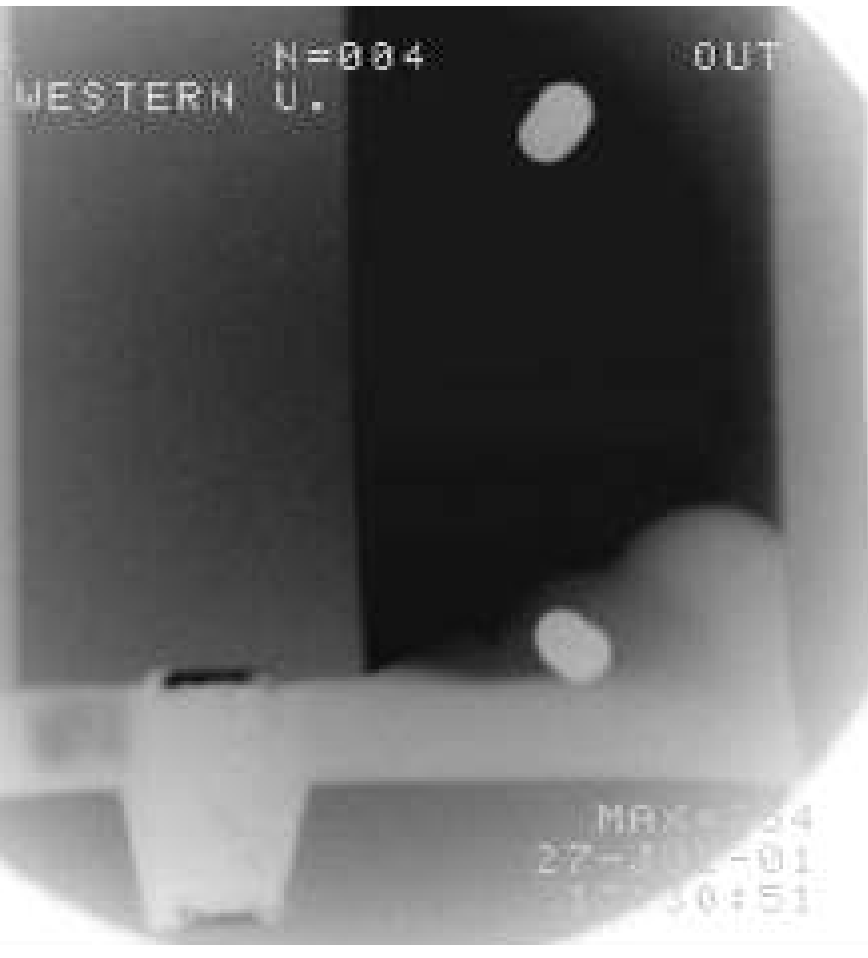

$0.60 \mathrm{sec}$
Without Filter

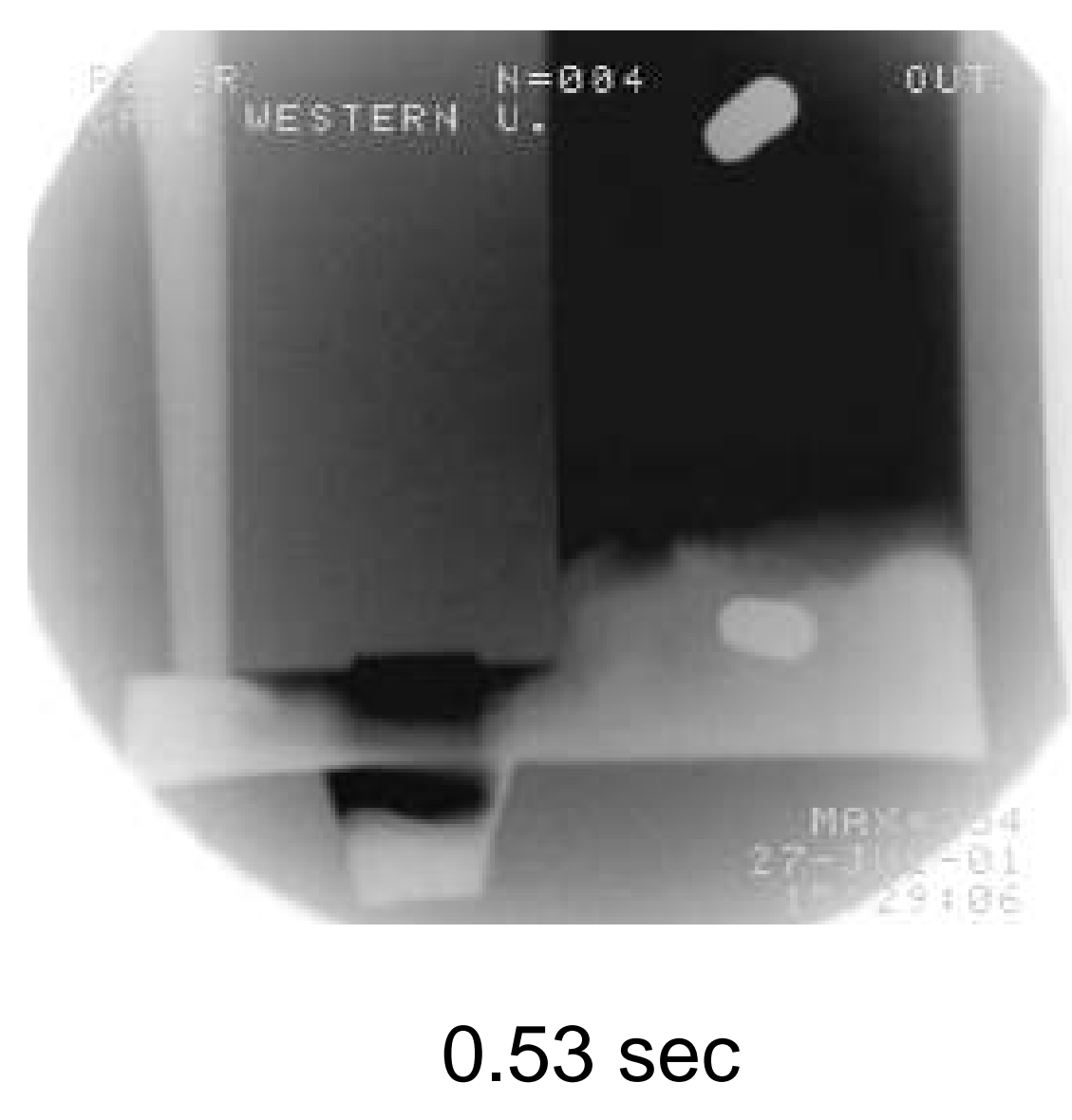


Figure 52f: Real-Time X-ray in Mold 9

10 ppi Filter

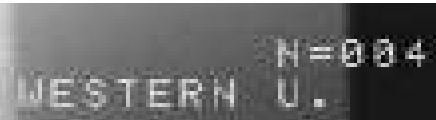

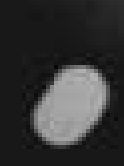

$0.83 \mathrm{sec}$

$1.23 \mathrm{sec}$

Without Filter

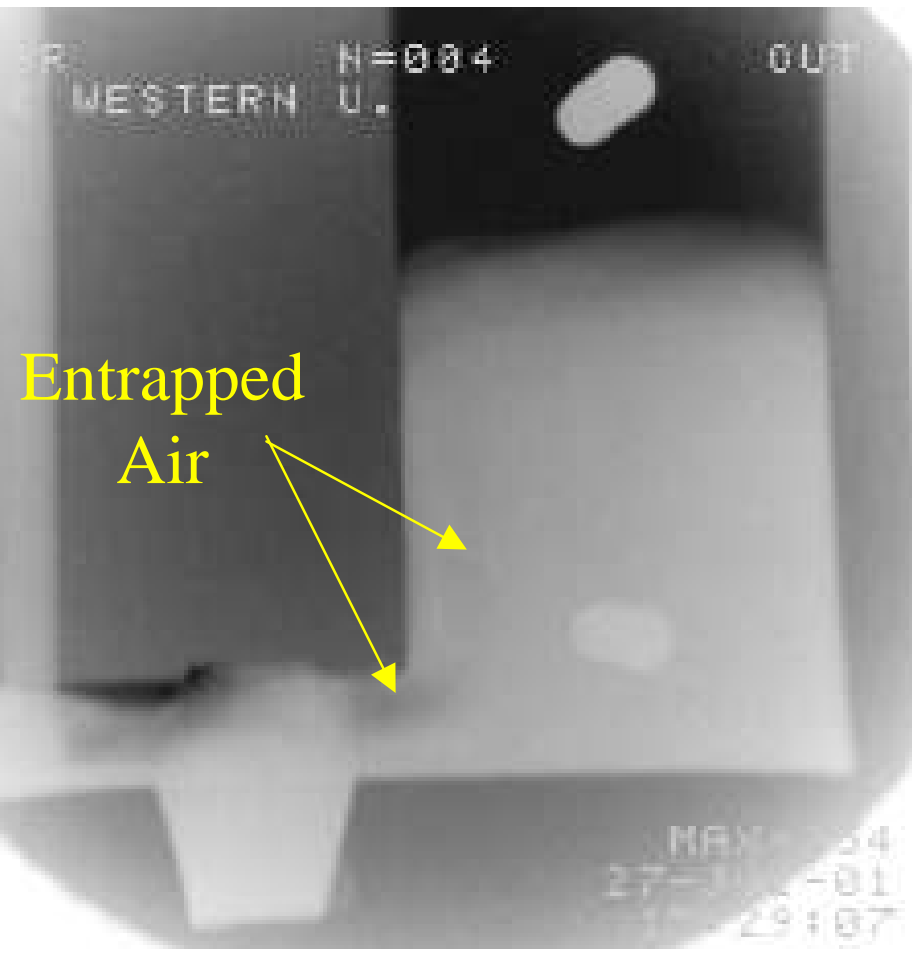


Figure 53a: Comparison of Simulation Results to Experiment In Mold 9

$$
\text { X-ray }
$$

WESTERH U. $\mathrm{N}=0.4$

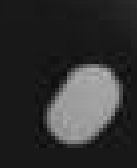

$0.37 \mathrm{sec}$
Simulation

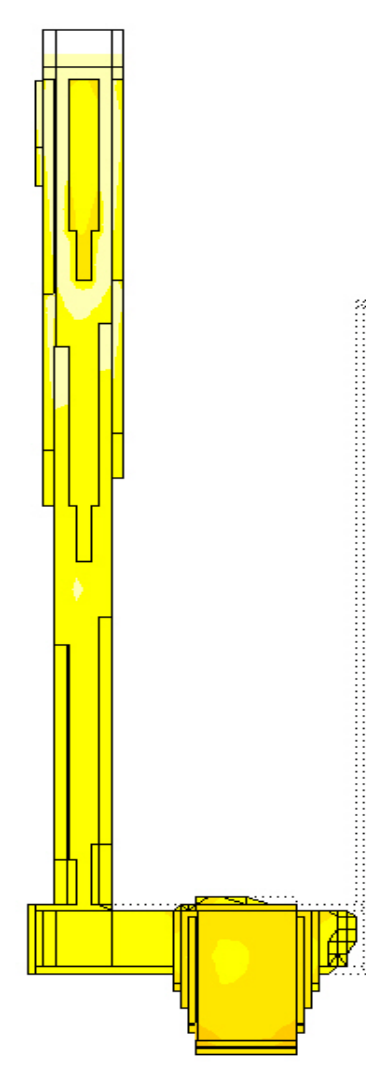

$0.4 \mathrm{sec}$ 
Figure 53b: Comparison of Simulation Results to Experiment In Mold 9

$$
\text { X-ray }
$$

$N=084$

HESTERH U.

$0.60 \mathrm{sec}$
Simulation

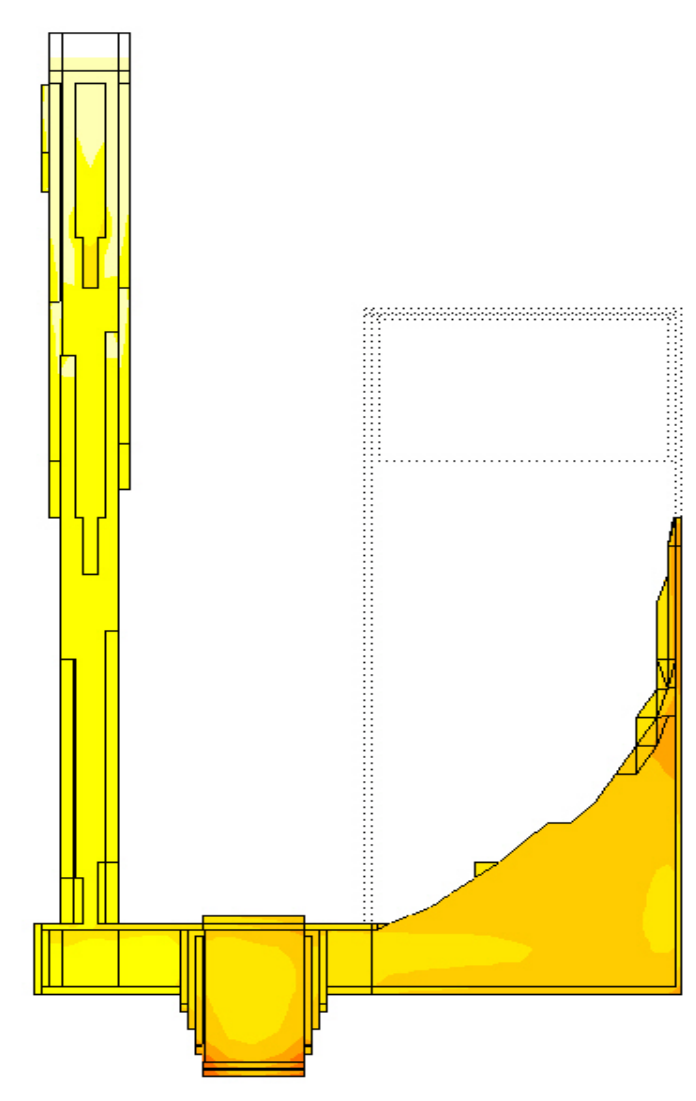

$0.7 \mathrm{sec}$ 
Figure 53c: Comparison of Simulation Results to Experiment In Mold 9

$$
\text { X-ray }
$$

$0.83 \mathrm{sec}$
Simulation

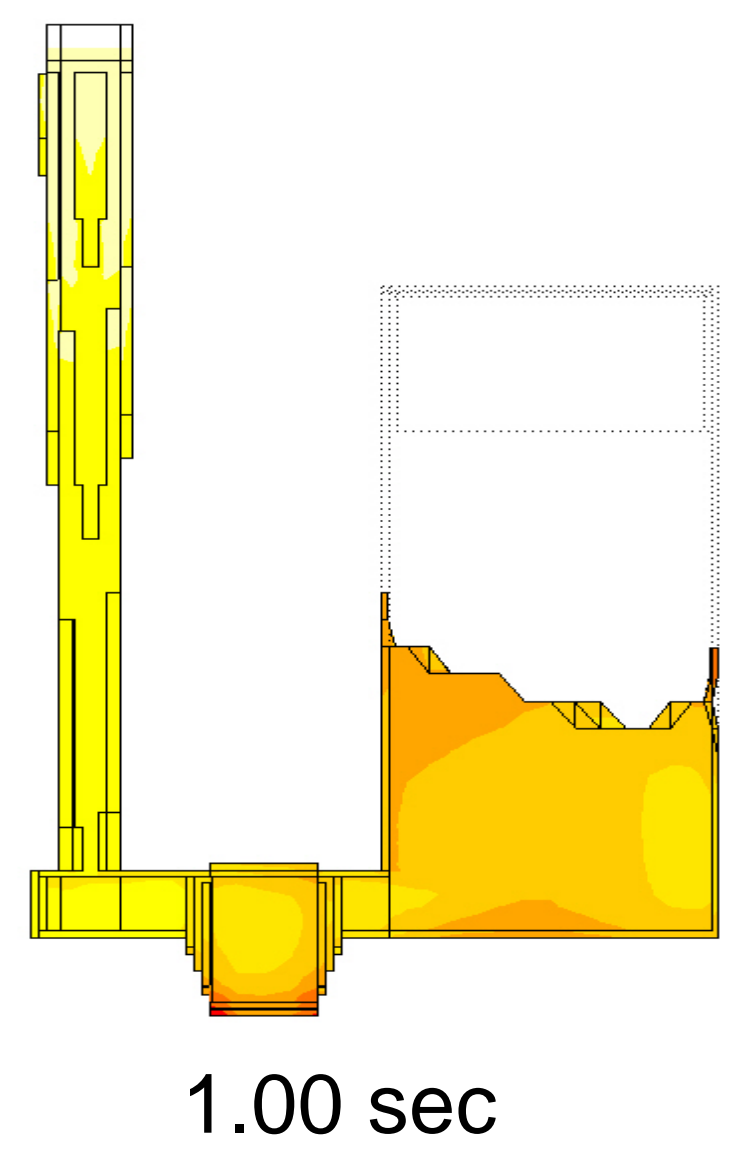




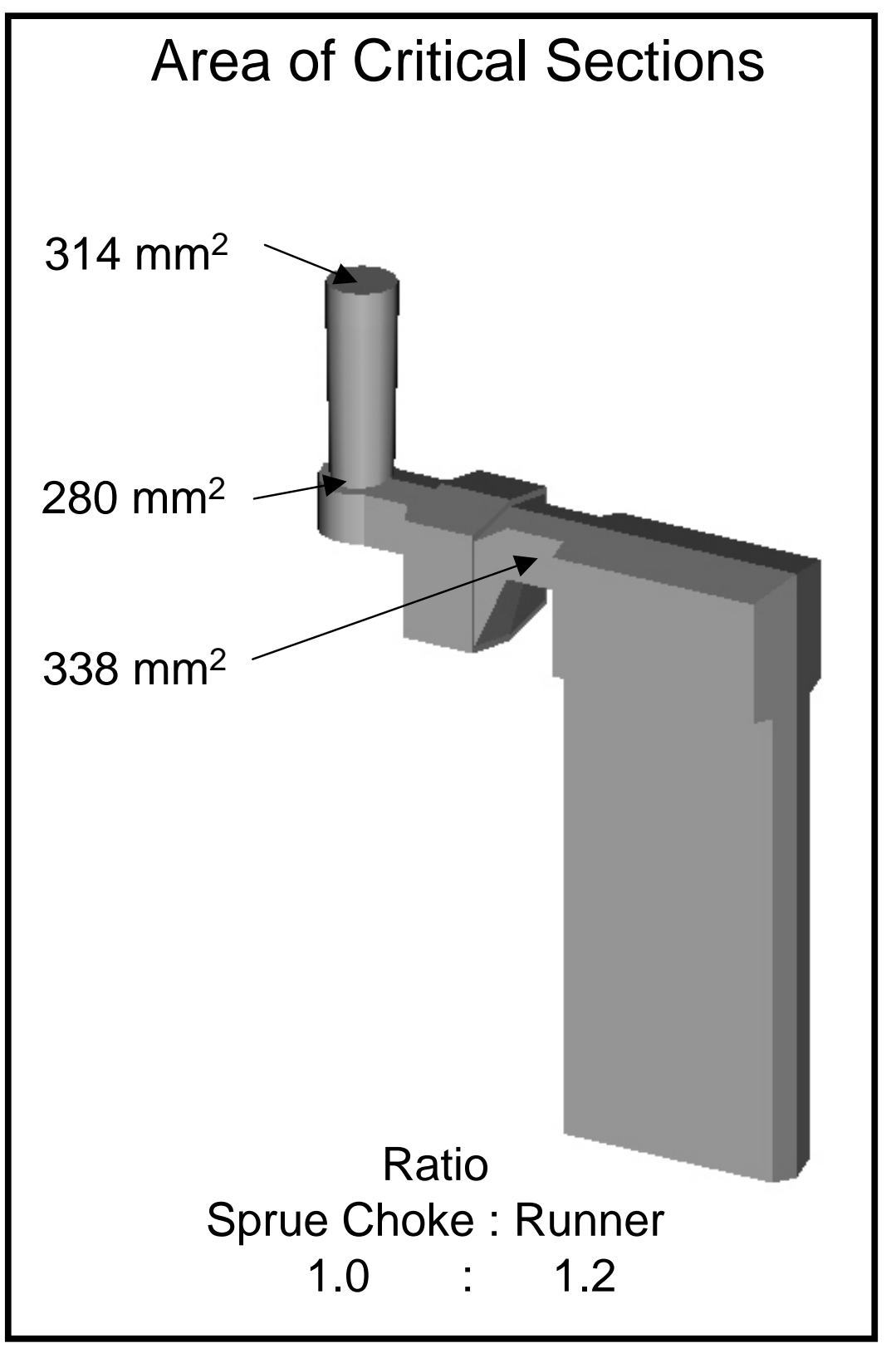

\section{Figure 54}

MOLD 10-Top Side Gate

- Side top gating system with a vertical $10 \mathrm{ppi}$ ceramic foam filter in the runner

- The ppi of the filter has little effect on the flow pattern.

- Filter makes the flow in the runner with positive pressure, avoids entrapping air into the mold. 
Figure 55: Mold 10-1 (with 10 ppi Filter)- Movie (click to view)

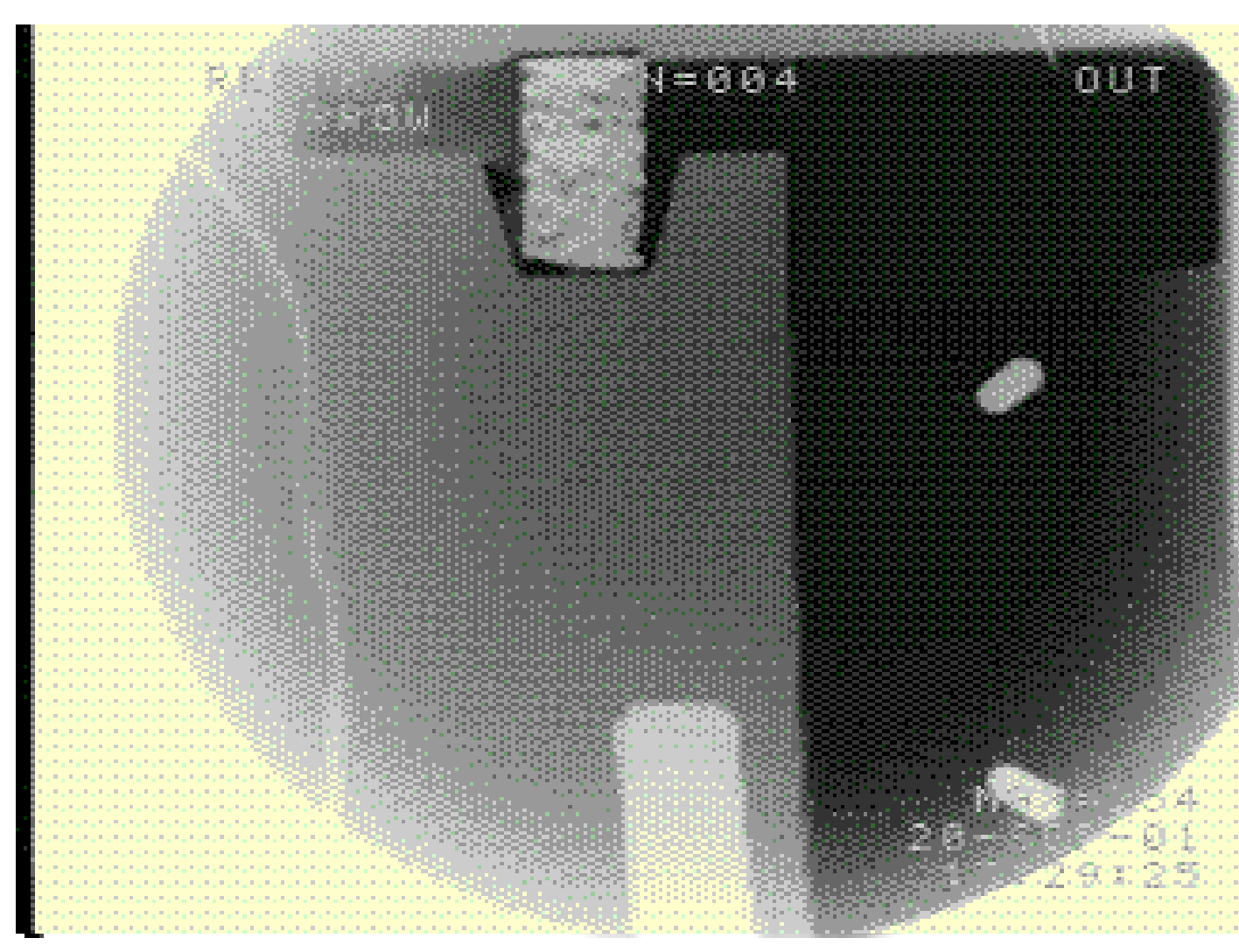


Figure 56: Mold10-2 (without Filter)- Movie (click to view)

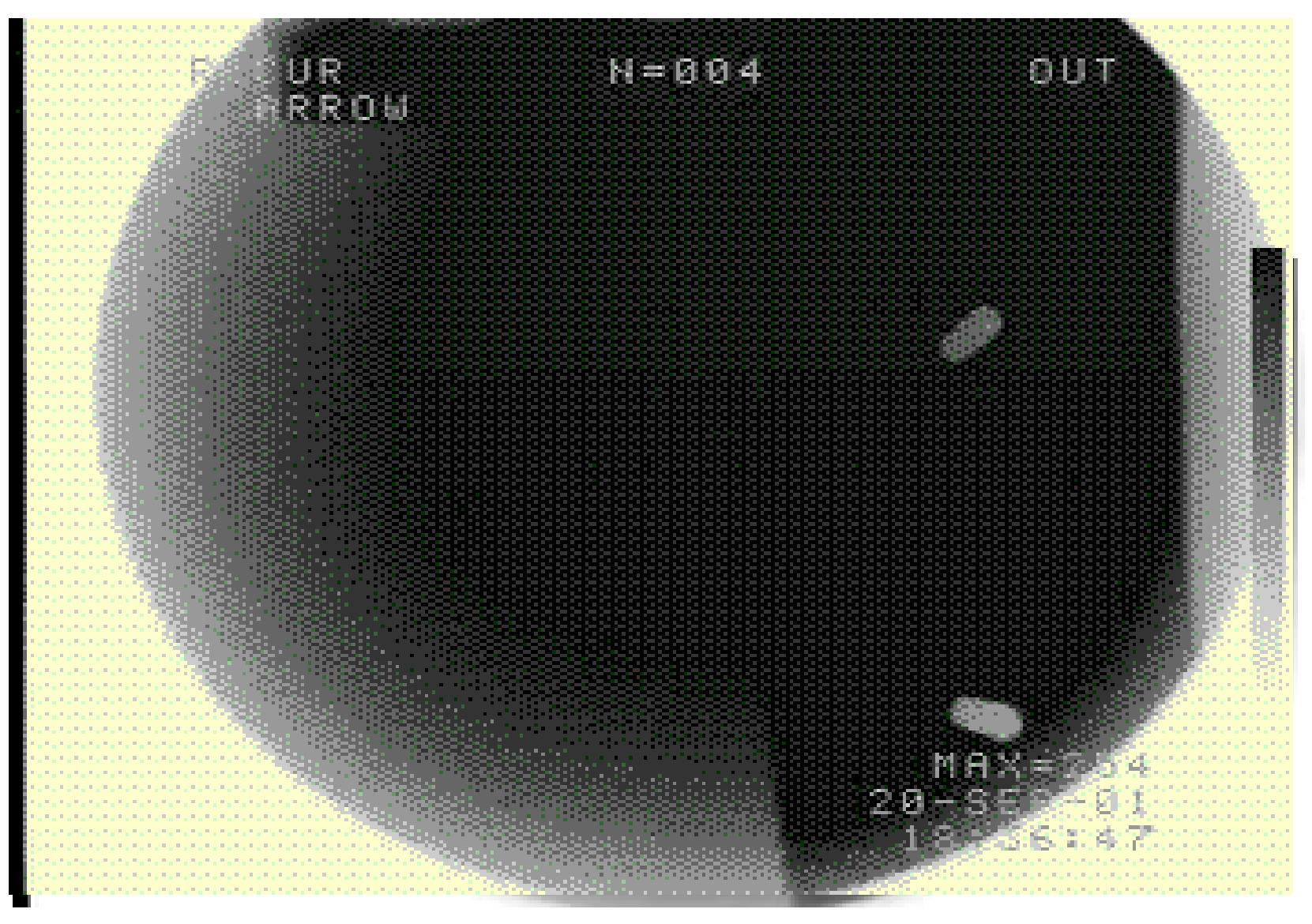


Figure 57a: Real-Time X-ray in Mold 10

\section{0 ppi Filter}

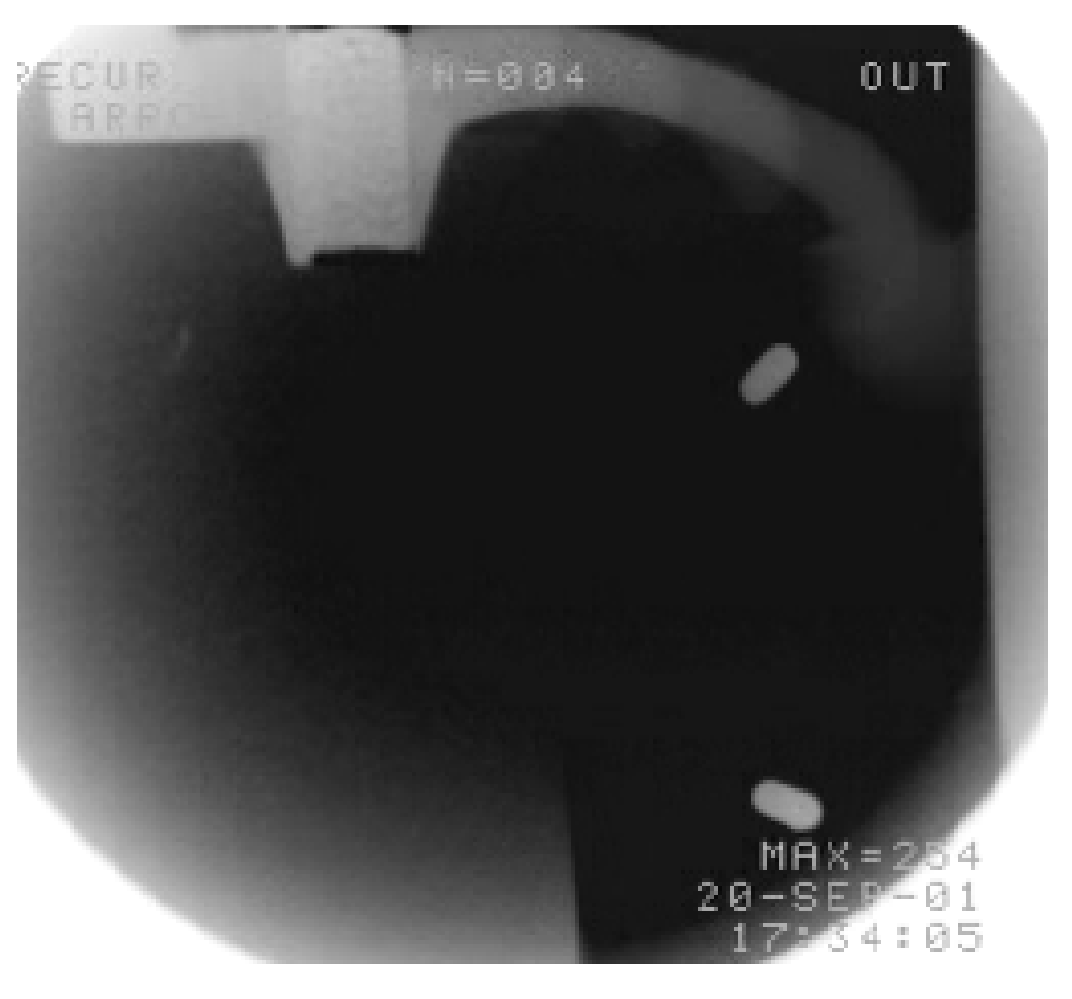

$0.40 \mathrm{sec}$
Without Filter

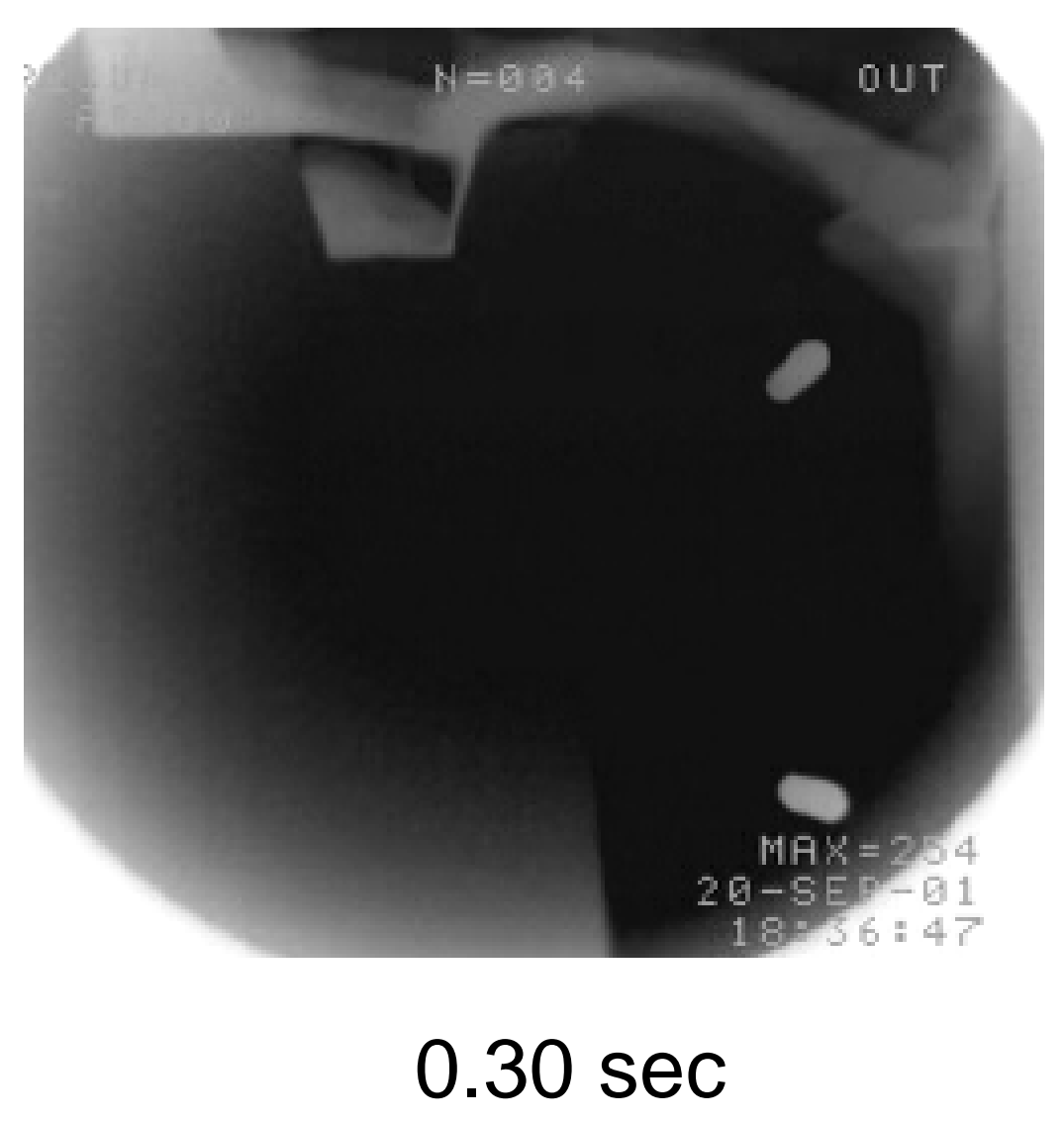


Figure 57b: Real-Time X-ray in Mold 10

\section{0 ppi Filter}

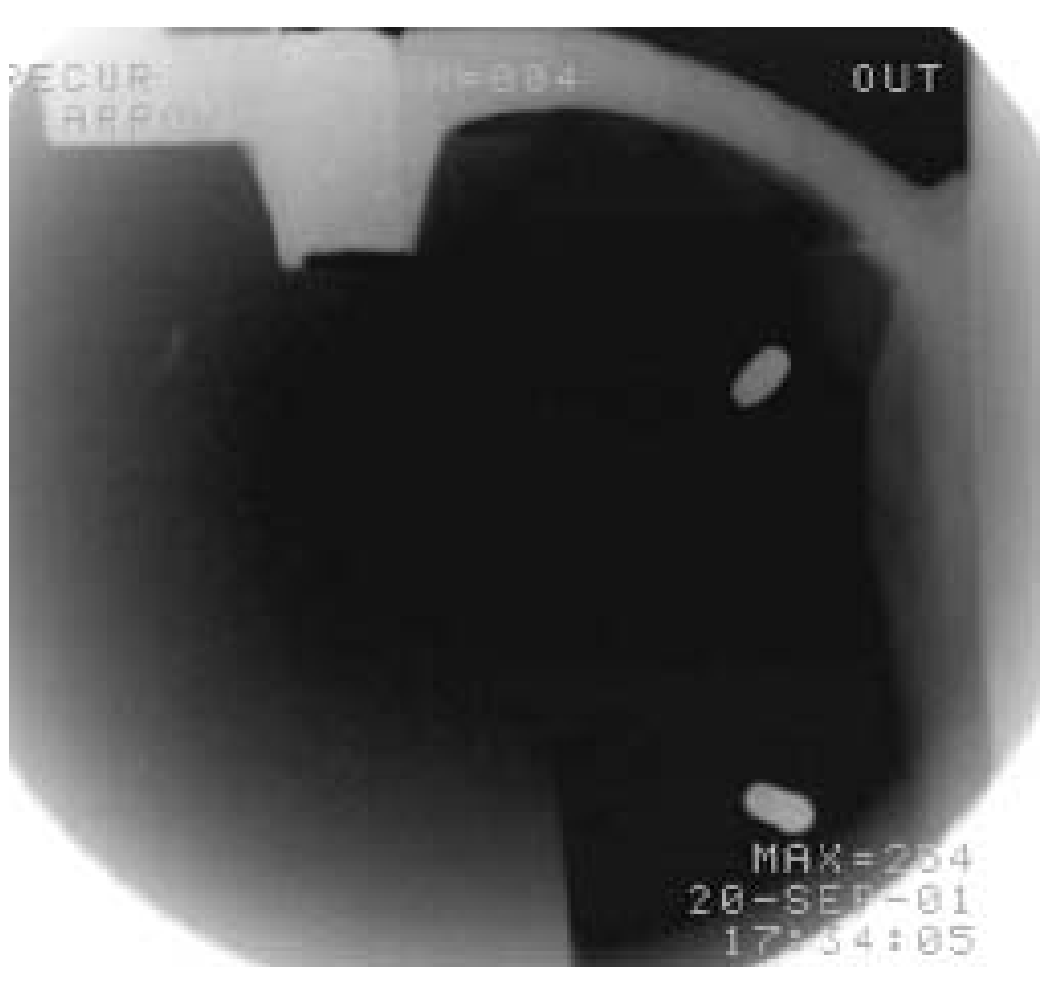

$0.53 \mathrm{sec}$
Without Filter

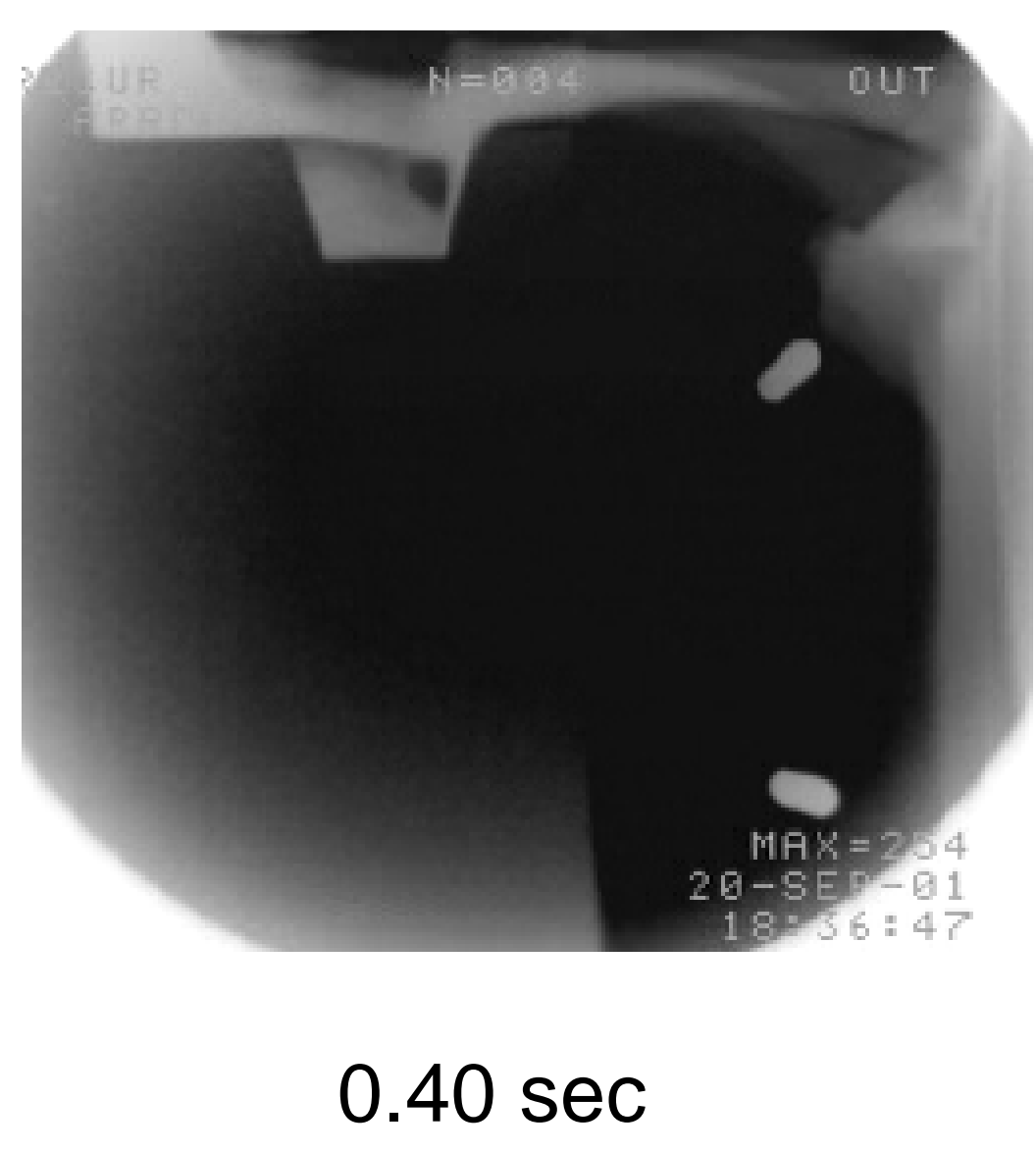


Figure 57c: Real-Time X-ray in Mold 10

\section{0 ppi Filter}

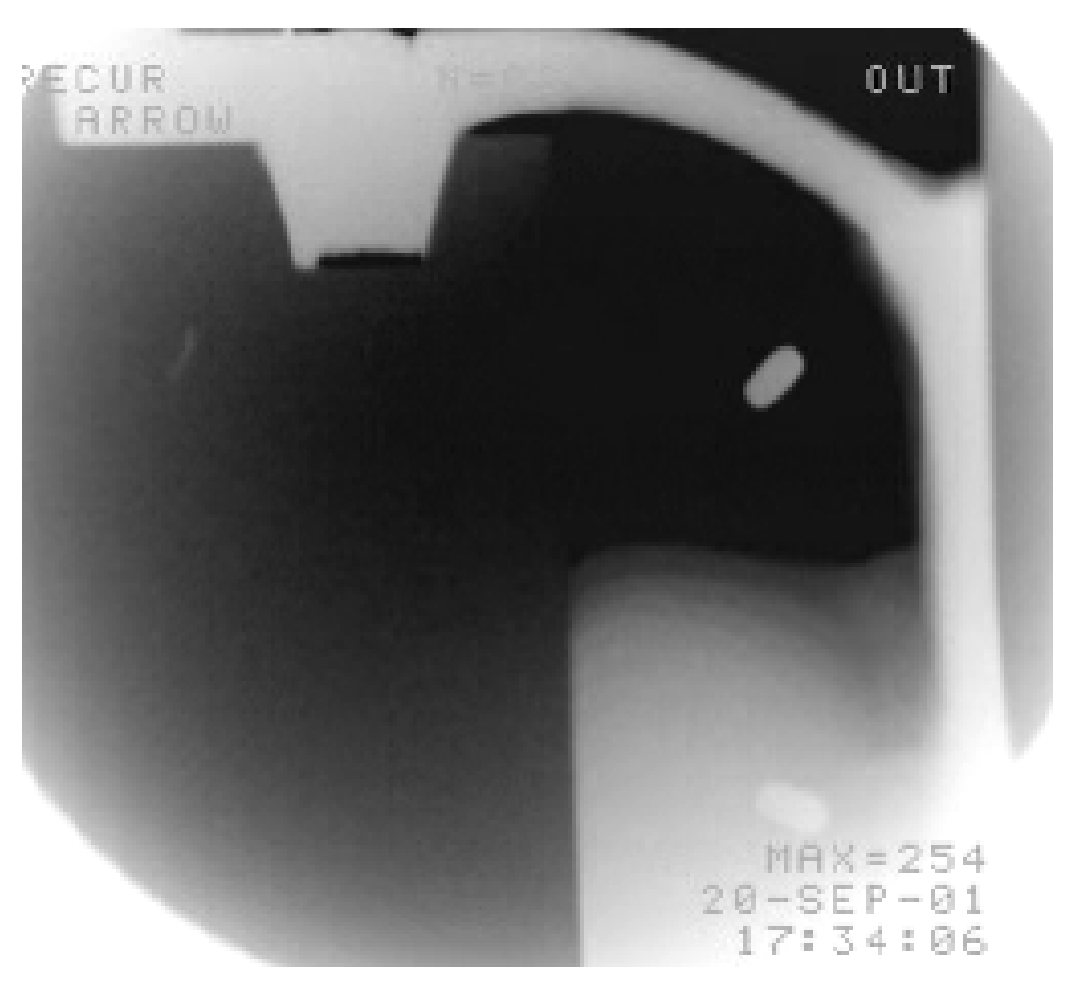

$1.13 \mathrm{sec}$
Without Filter

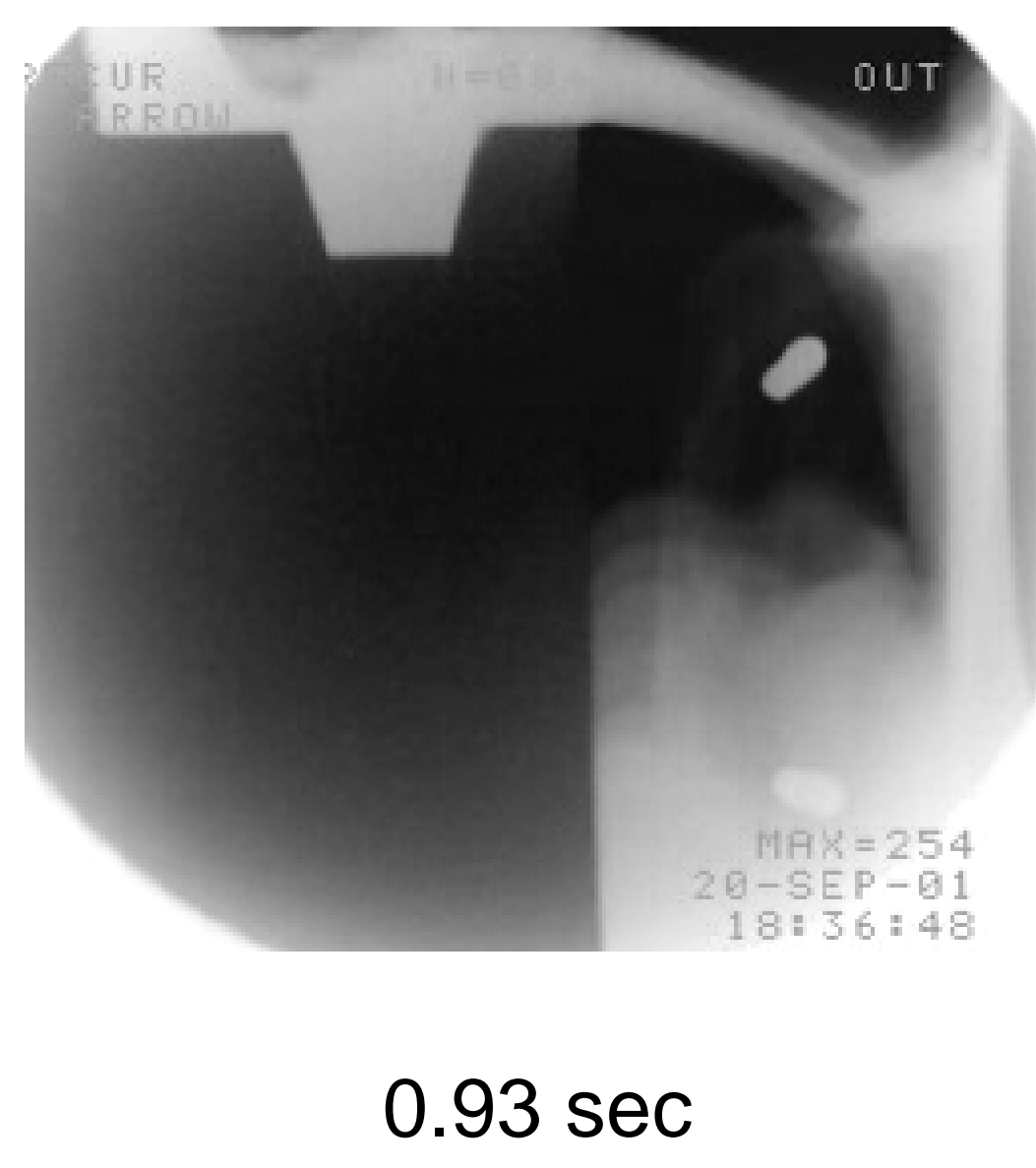


Figure 57d: Real-Time X-ray in Mold 10

\section{0 ppi Filter}

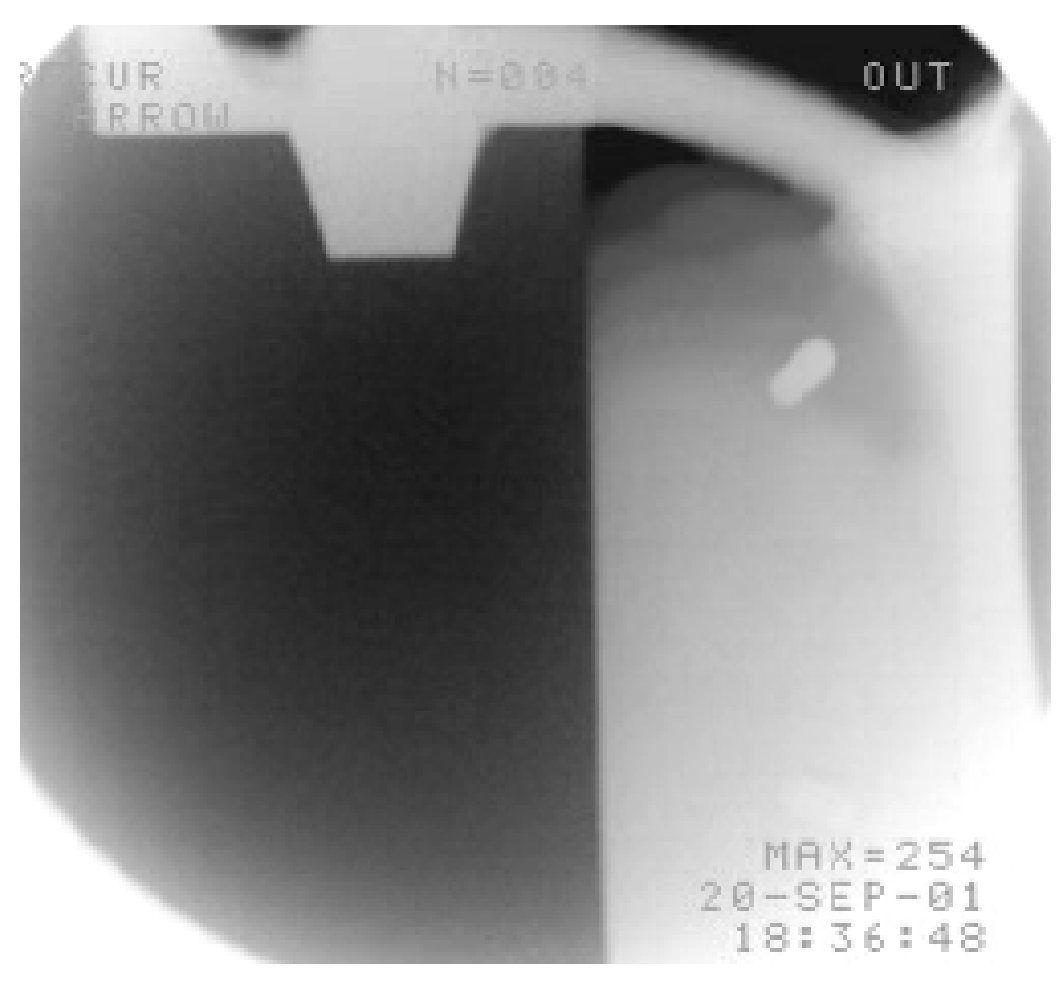

$1.13 \mathrm{sec}$
Without Filter

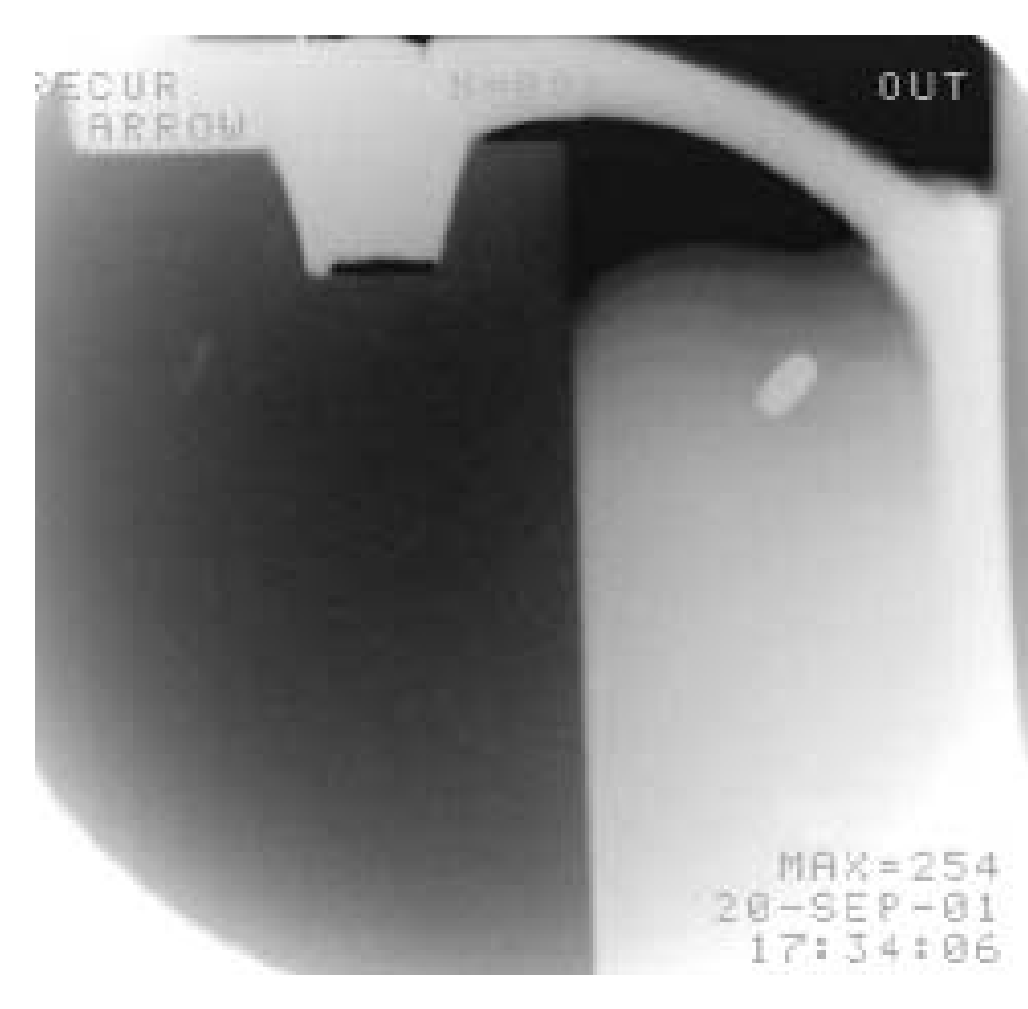

$1.46 \mathrm{sec}$ 
Figure 57e: Real-Time X-ray in Mold 10

\section{0 ppi Filter}

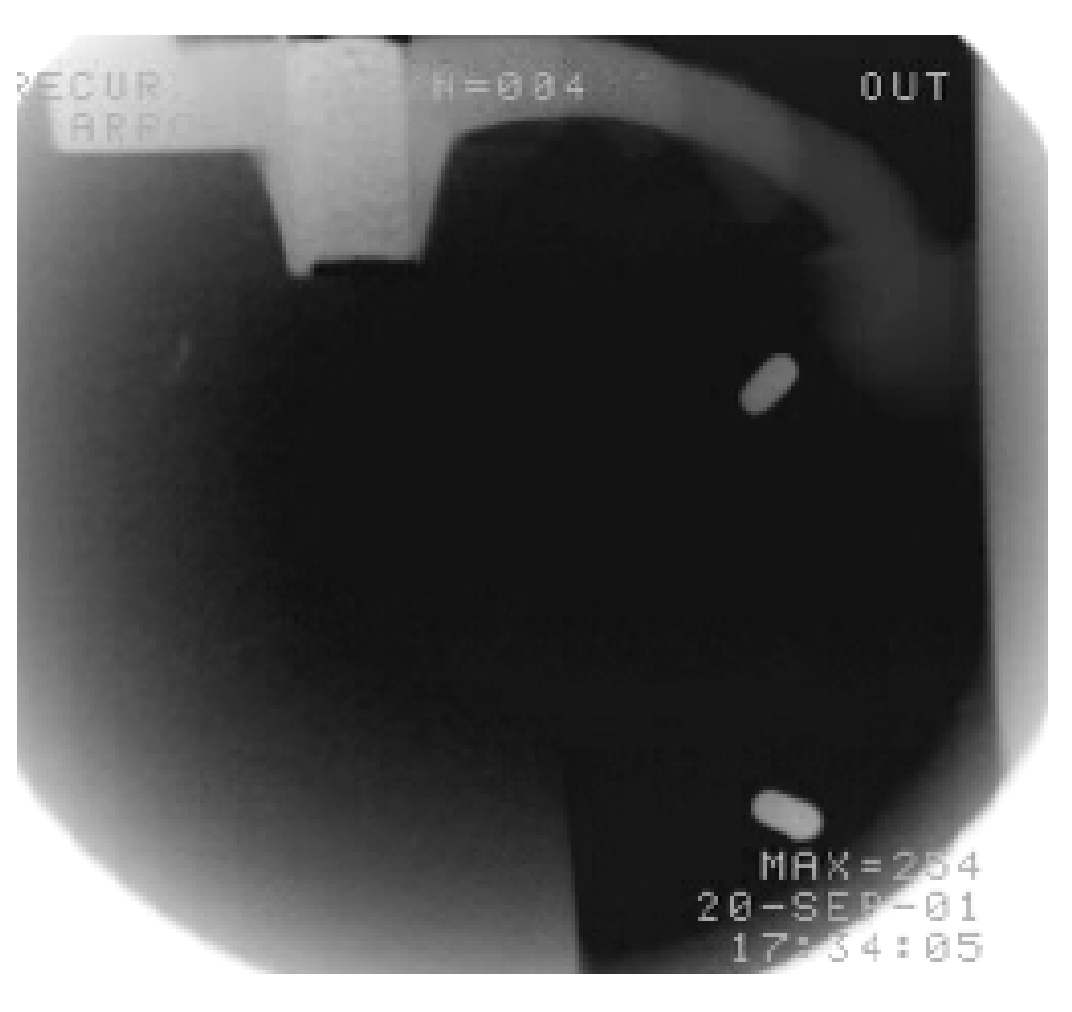

$0.40 \mathrm{sec}$

\section{Simulation}

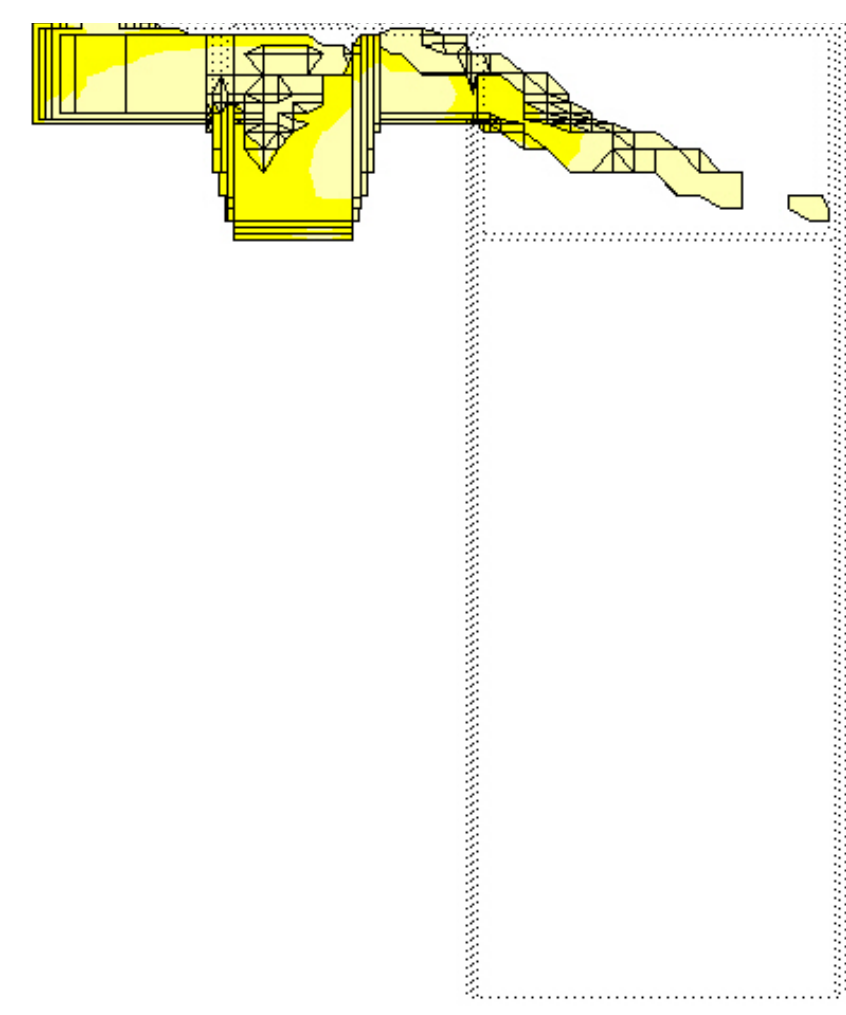

$0.30 \mathrm{sec}$ 
Figure 57f: Real-Time X-ray in Mold 10

\section{0 ppi Filter}

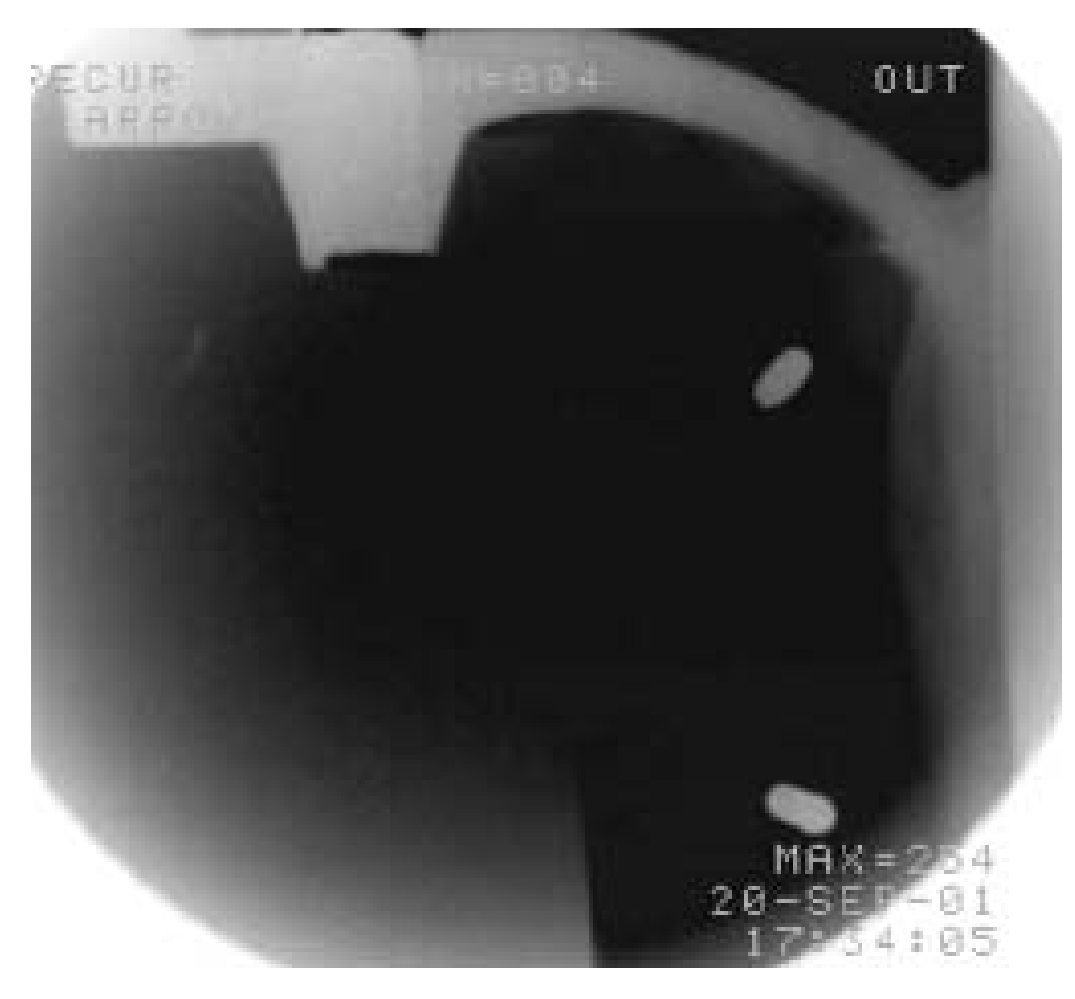

$0.53 \mathrm{sec}$
Simulation

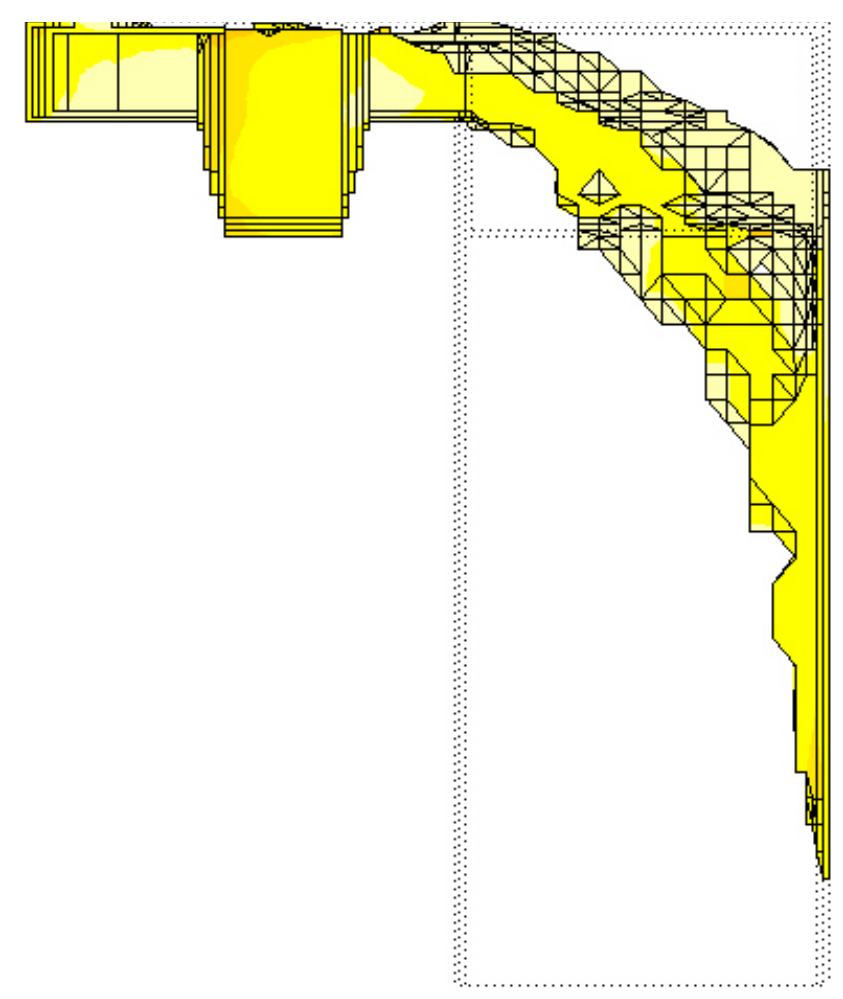

$0.50 \mathrm{sec}$ 
Figure 57g: Real-Time X-ray in Mold 10

\section{0 ppi Filter}

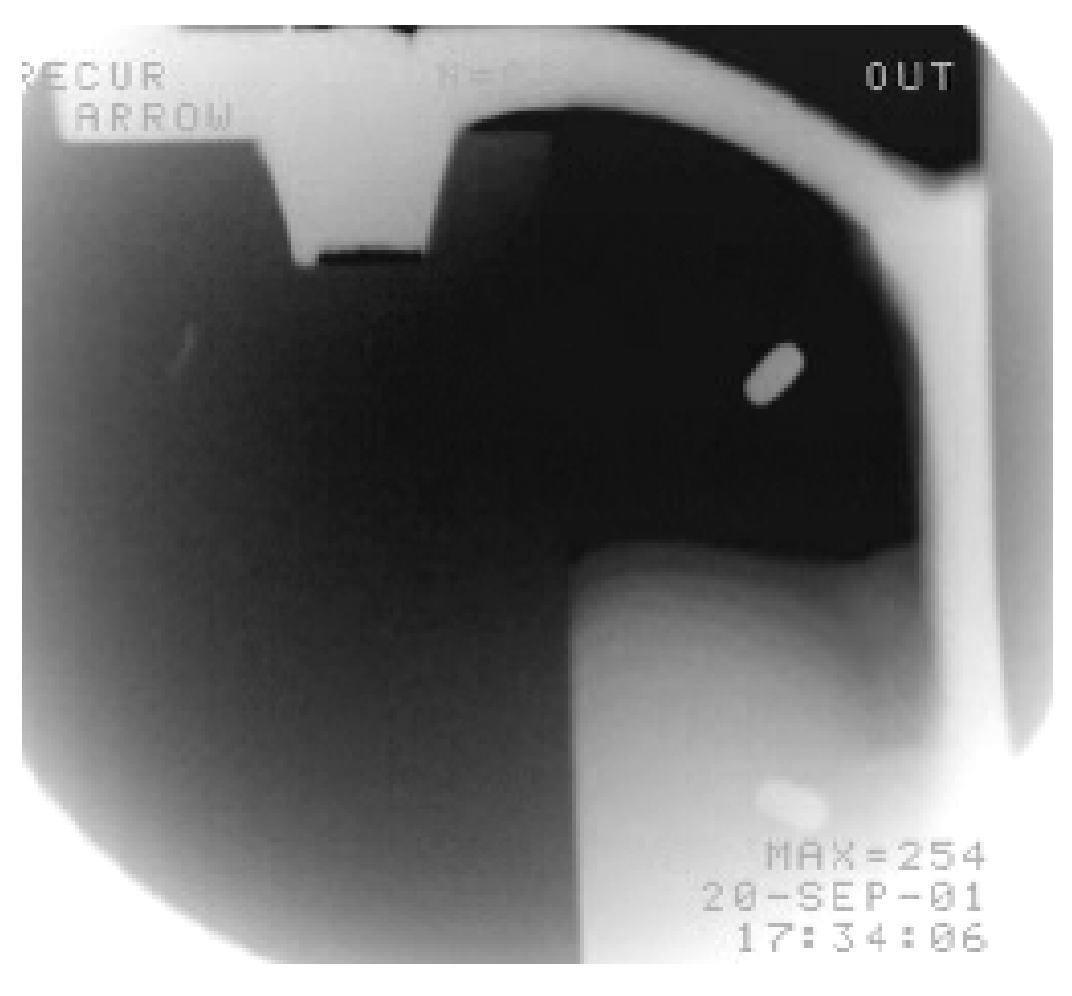

$1.13 \mathrm{sec}$
Simulation

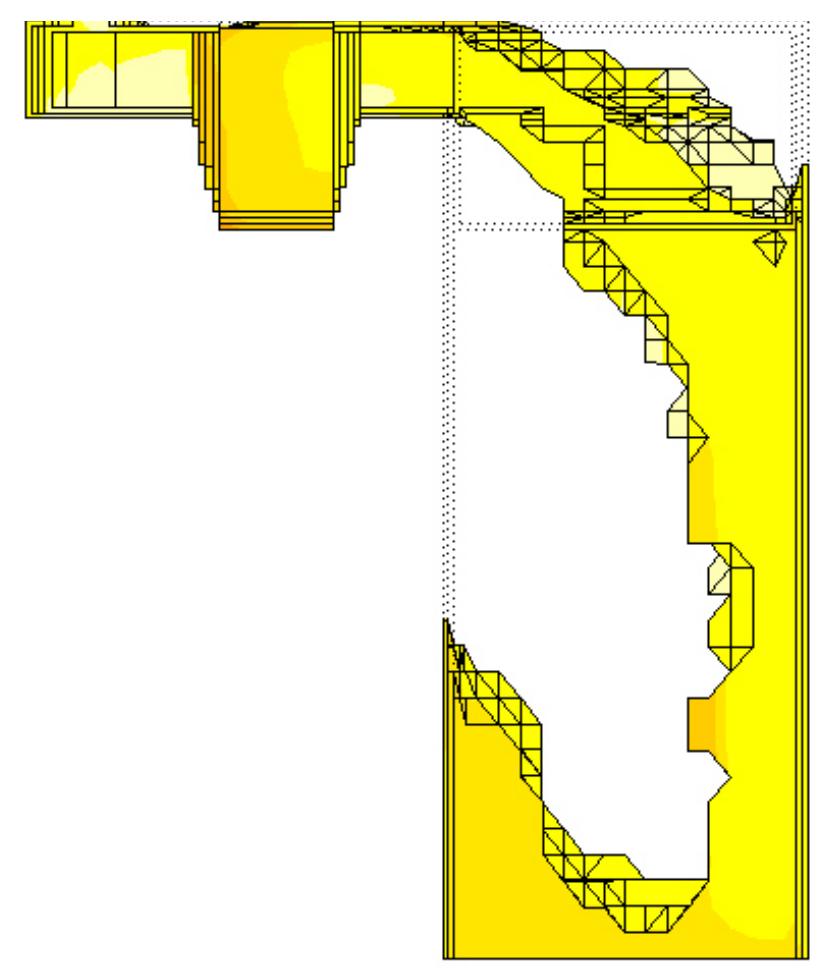

$1.10 \mathrm{sec}$ 
Figure 57h: Real-Time X-ray in Mold 10

\section{0 ppi Filter}

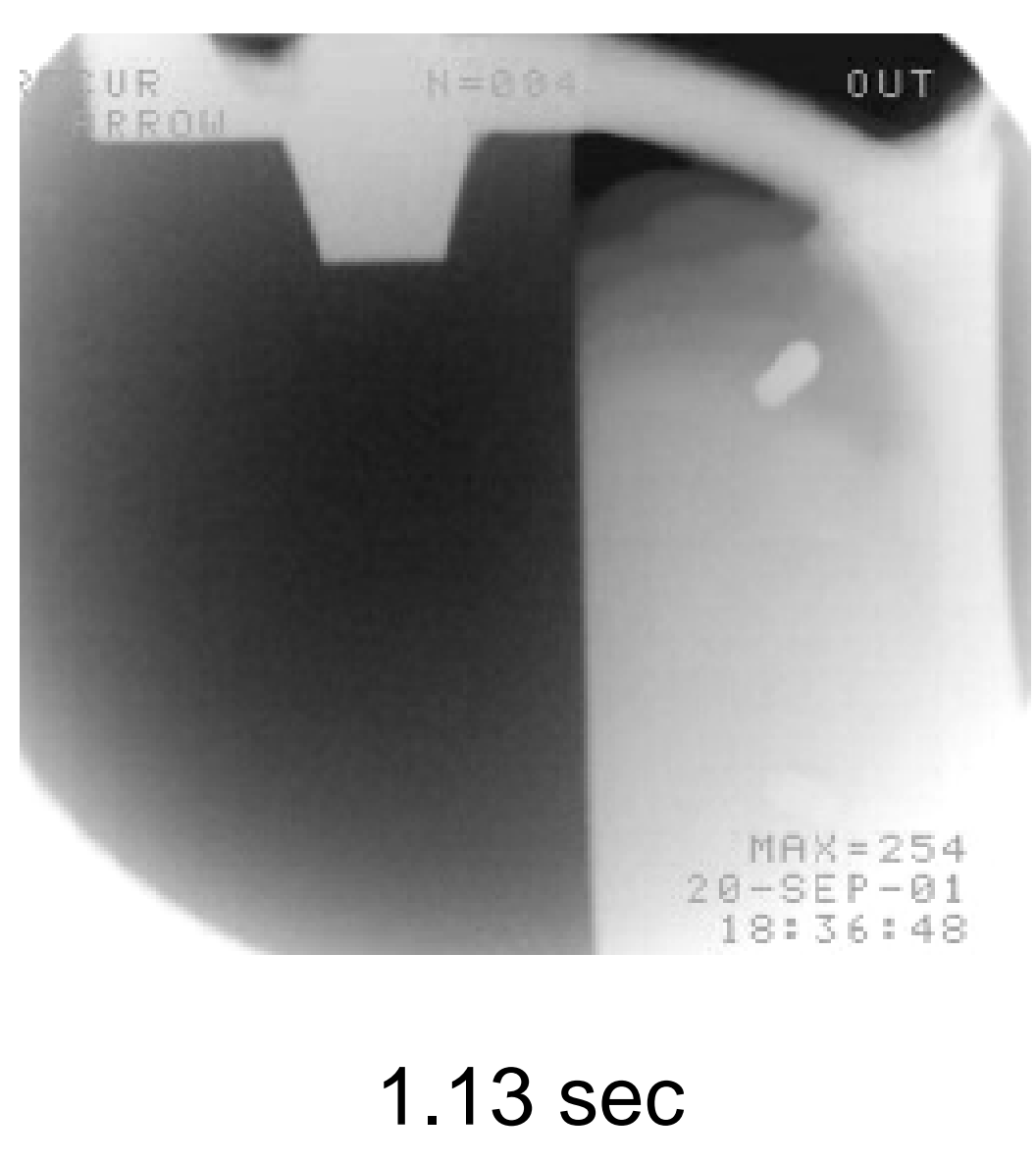

Simulation

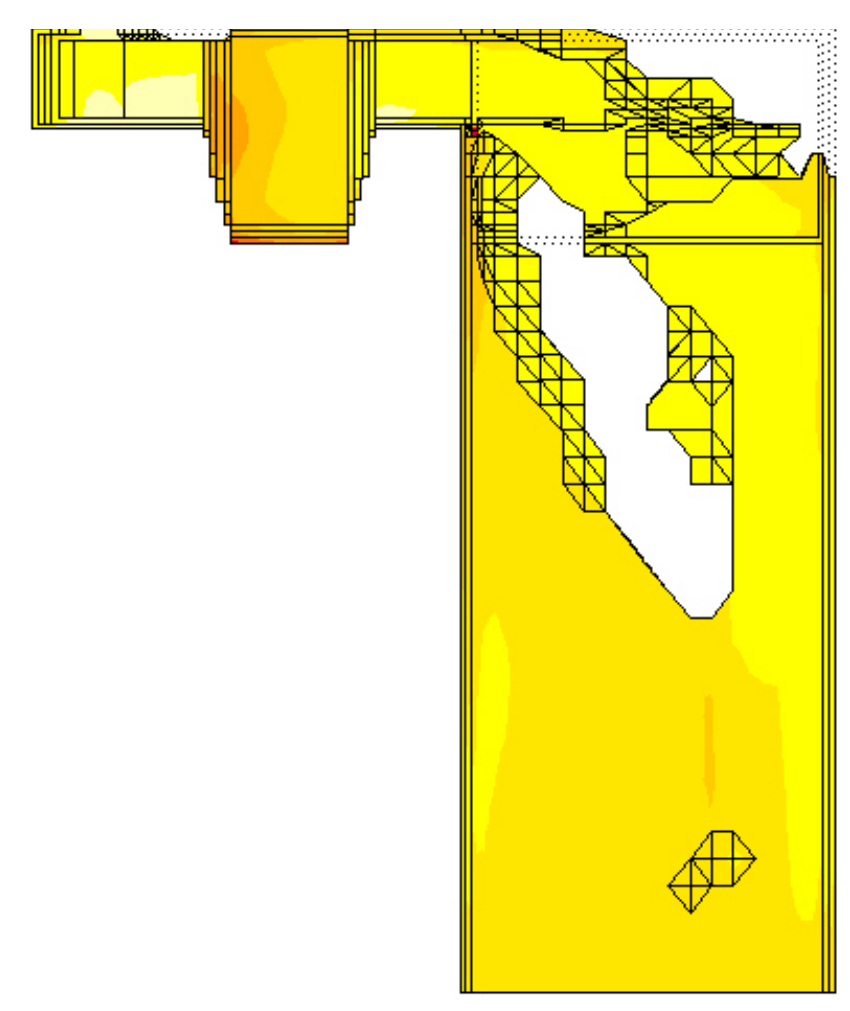

$1.2 \mathrm{sec}$ 


\section{Figure 58: Velocity and Fill Time}

\begin{tabular}{|c|c|c|c|c|c|}
\hline & & Vc(in/sec) & $\operatorname{Vr}($ in/sec$)$ & Vi(in/sec) & Fill Time (sec) \\
\hline \multirow{3}{*}{ mold 22} & Exp. & 57 & 24 & 19 & 0.8 \\
\hline & Cal. & 78 & 32.5 & 26.9 & I \\
\hline & Simu. & 63 & 21.5 & 12 & 0.9 \\
\hline \multirow{3}{*}{ mold22-2 } & Exp. & 79.8 & 20.45 & 16.27 & 0.92 \\
\hline & Cal. & 78 & 20 & 16.2 & I \\
\hline & Simu. & $I$ & $I$ & I & I \\
\hline \multirow{3}{*}{ mold13 } & Exp. & 55 & 8.8 & 8.5 & 1.3 \\
\hline & Cal. & 78 & 12.4 & 12 & I \\
\hline & Simu. & 123 & 12 & 17 & 0.9 \\
\hline \multirow{3}{*}{ mold31 } & Exp. & 81.2 & 17.8 & 7.7 & 1.4 \\
\hline & Cal. & 78 & 17 & 7.3 & $I$ \\
\hline & Simu. & 85 & 22 & 12 & 1.8 \\
\hline \multirow{3}{*}{ mold41 } & Exp. & 93.09 & 7.7 & 8.7 & 1.3 \\
\hline & Cal. & 77 & 8.1 & 7.2 & $I$ \\
\hline & Simu. & 1 & $I$ & $I$ & $I$ \\
\hline \multirow{3}{*}{ mold51 } & Exp. & 59 & 23 & 12 & 1.4 \\
\hline & Cal. & 78 & 31.2 & 15.6 & I \\
\hline & Simu. & 1 & 1 & 1 & $I$ \\
\hline \multirow{3}{*}{ mold52 } & Exp. & 43.1 & 17.25 & 8.6 & 1.2 \\
\hline & Cal. & 78 & 31.2 & 15.6 & $I$ \\
\hline & Simu. & $I$ & I & $I$ & I \\
\hline
\end{tabular}

Comments:

- Vc, Velocity in sprue choke $\quad V r$, Velocity in runner

- Fill time to 2.8 inches above gate, visible in X-Ray frame

- Vi, Velocity in ingate $\quad$ * Velocity in riser

- Calculated velocities based on Bernoulli equation 


\section{Figure 58: Velocity and Fill Time}

\begin{tabular}{|c|c|c|c|c|c|}
\hline & & Vc(in/sec) & $\mathrm{Vr}$ (in/sec) & Vi(in/sec) & Fill Time (sec) \\
\hline \multirow{3}{*}{ m 8} & Exp. & 37.10 & 7.42 & I & 1.6 \\
\hline & Cal. & 78.9 & 15.78 & I & $I$ \\
\hline & Simu. & 41.58 & 8.316 & I & 1.23 \\
\hline \multirow{3}{*}{ m 9-1 } & Exp. & 56.77 & 13.10 & $I$ & 1.2 \\
\hline & Cal. & 75.5 & 17.65 & $I$ & $I$ \\
\hline & Simu. & 70.89 & 16.15 & $I$ & 1.12 \\
\hline \multirow{3}{*}{ m 9-3(w/o) } & Exp. & 60.98 & 14.70 & $I$ & 1.2 \\
\hline & Cal. & $I$ & I & $I$ & $I$ \\
\hline & Simu. & 75.67 & 18.52 & $I$ & 0.96 \\
\hline \multirow{3}{*}{ m 10-1 } & Exp. & 36.05 & 29.87 & $I$ & 0.9 \\
\hline & Cal. & 71.9 & 59.57 & $I$ & $I$ \\
\hline & Simu. & 33.34 & 27.65 & $I$ & 1.01 \\
\hline \multirow{3}{*}{ m 10-2(w/o } & Exp. & 38.84 & 32.17 & $I$ & 0.8 \\
\hline & Cal. & $I$ & $I$ & $I$ & $I$ \\
\hline & Simu. & 41.96 & 34.75 & I & 1.74 \\
\hline & & & & & \\
\hline & & & & & \\
\hline & & & & & \\
\hline & & & & & \\
\hline & & & & & \\
\hline & & & & & \\
\hline
\end{tabular}

Comments:

- Vc, Velocity in sprue choke $\quad \mathrm{Vr}$, Velocity in runner

- Fill time to 2.8 inches above gate, visible in X-Ray frame
- Vi, Velocity in ingate $\quad *$ * Velocity in riser

- Calculated velocities based on Bernoulli equation 


\section{Figure 60: Arrow Box -Animation (click to view)}

\section{Velocity Field}

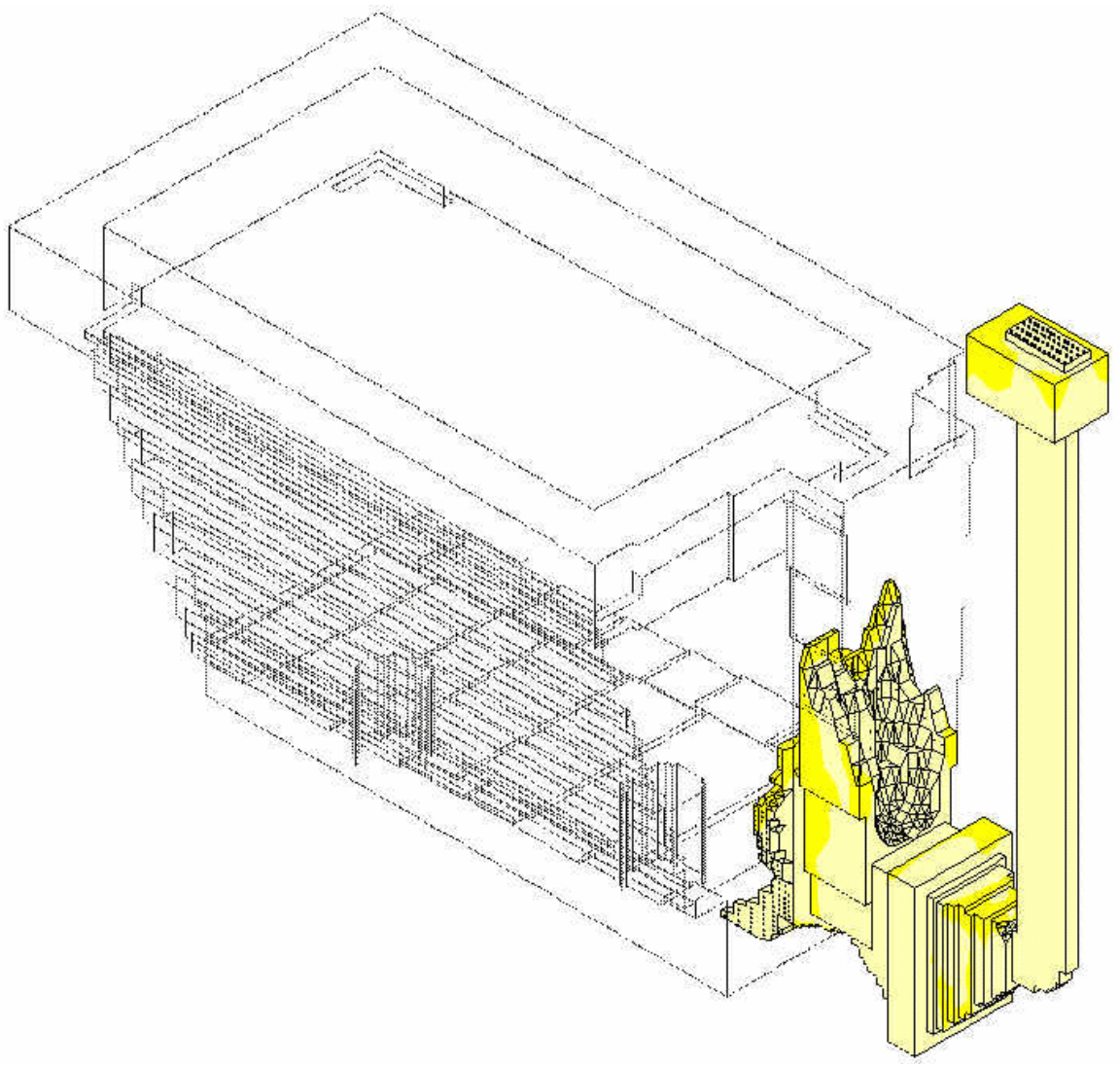

Particle Tracing

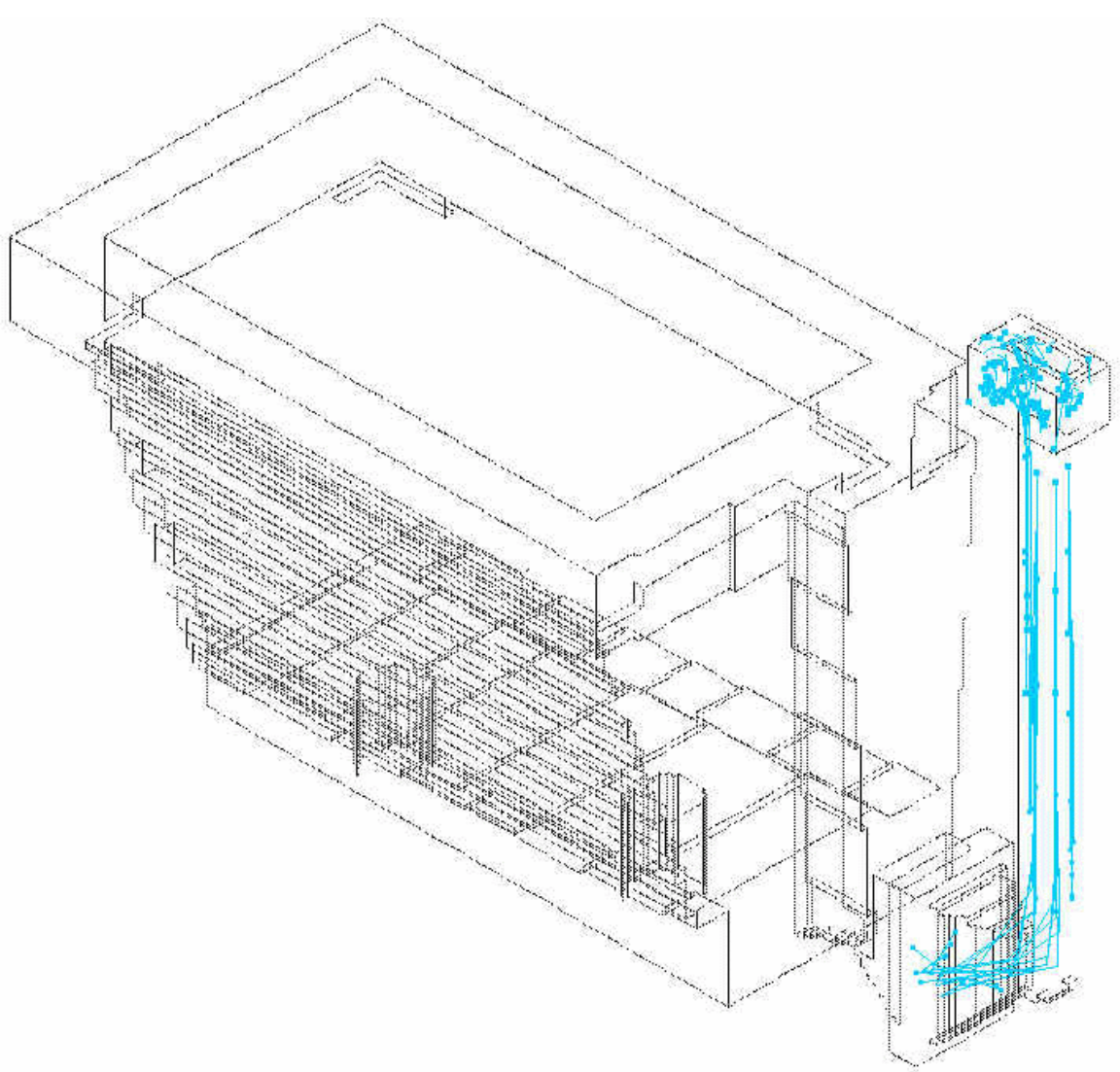


Figure 61 : Simulation Results of the "Box Casting"

(a) Flow Pattern - Front View

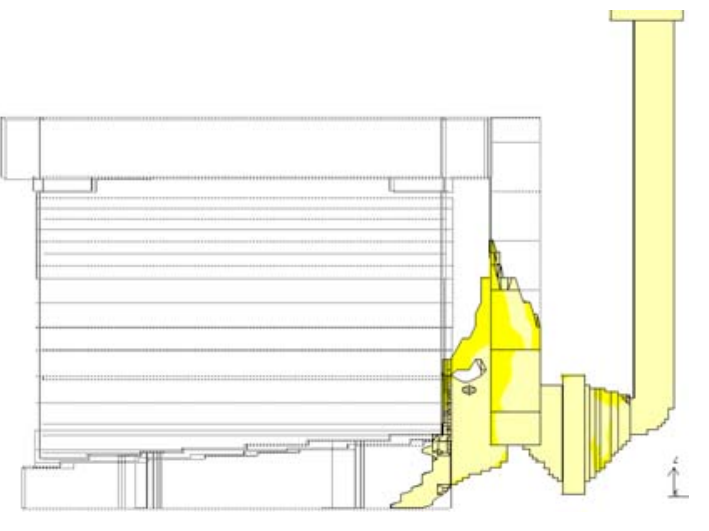

$0.8 \mathrm{sec}$

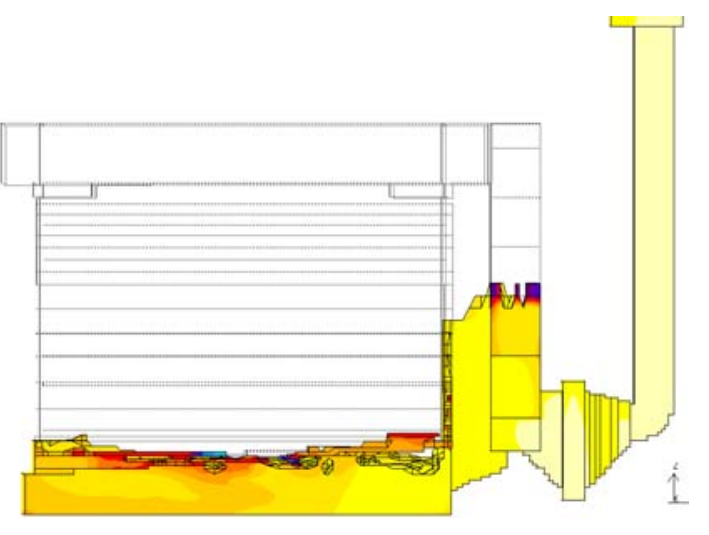

$3.2 \mathrm{sec}$

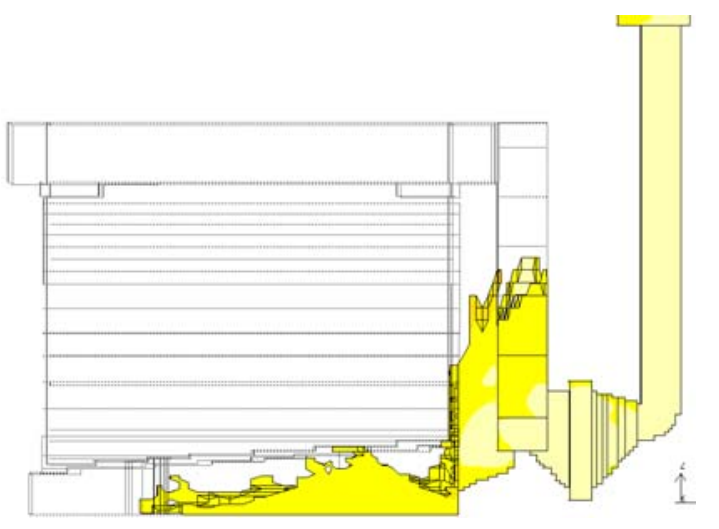

$1.2 \mathrm{sec}$

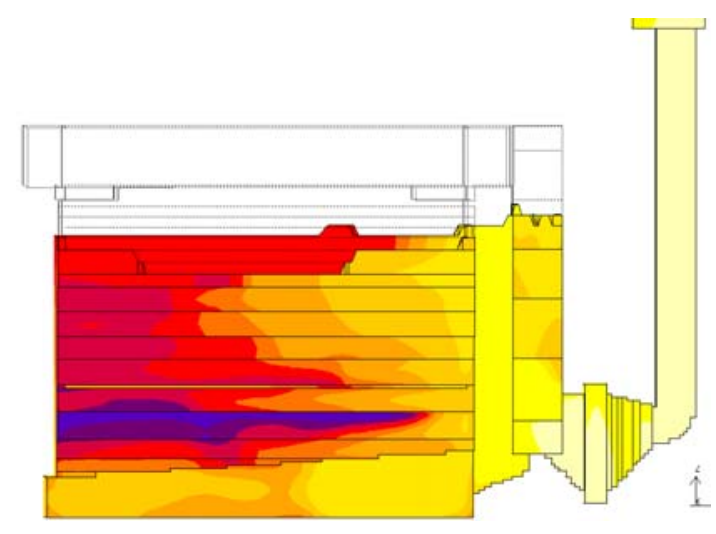

$6.0 \mathrm{sec}$

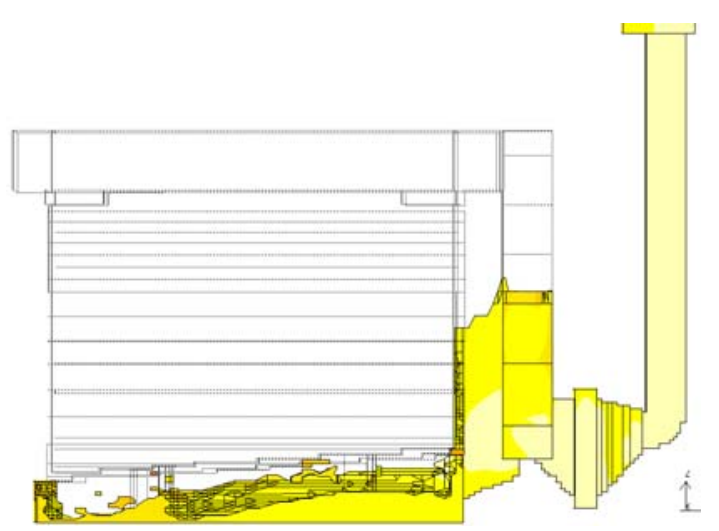

$1.6 \mathrm{sec}$

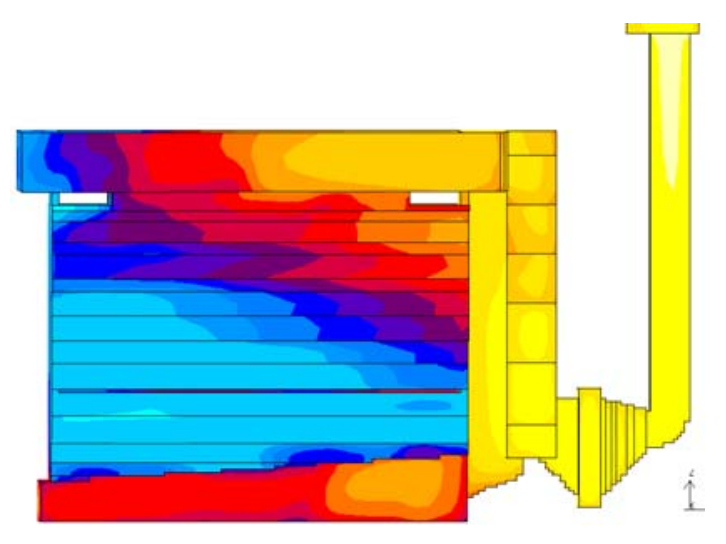

$8.0 \mathrm{sec}$ 
Figure 61 : Simulation Results of the "Box Casting"

\section{(b) Flow Pattern - 3D Perspective}

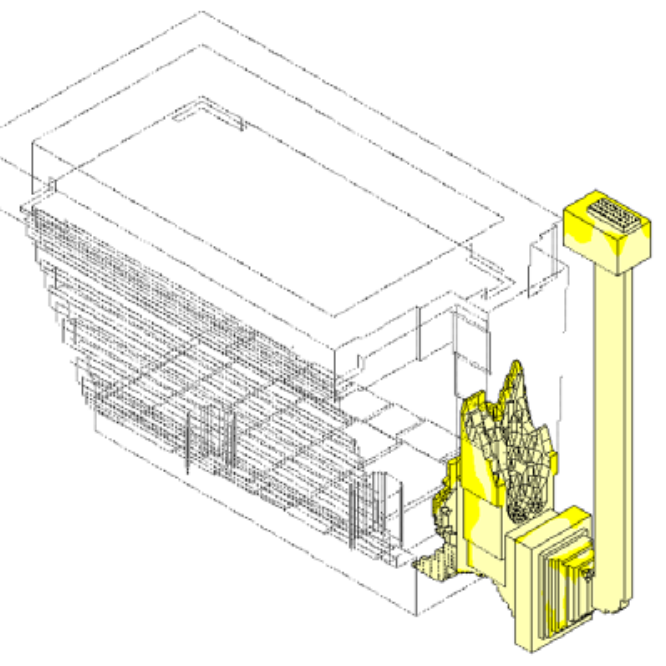

$0.8 \mathrm{sec}$

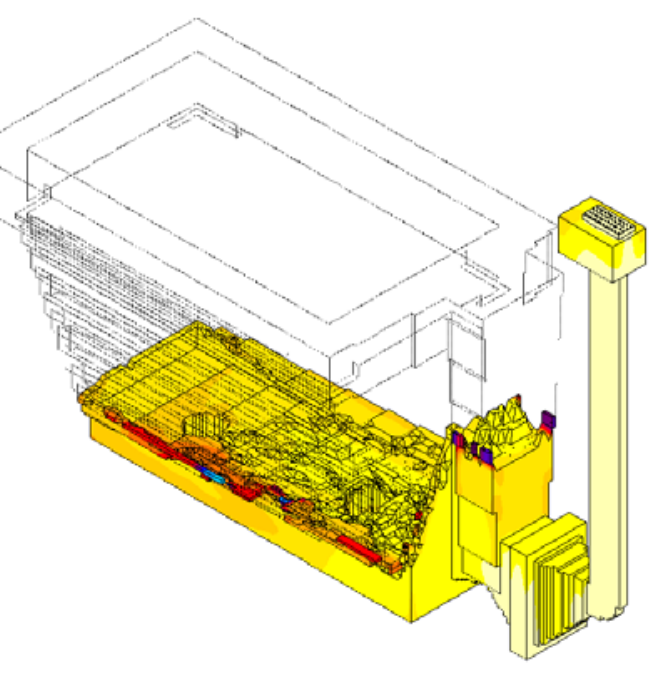

$3.2 \mathrm{sec}$

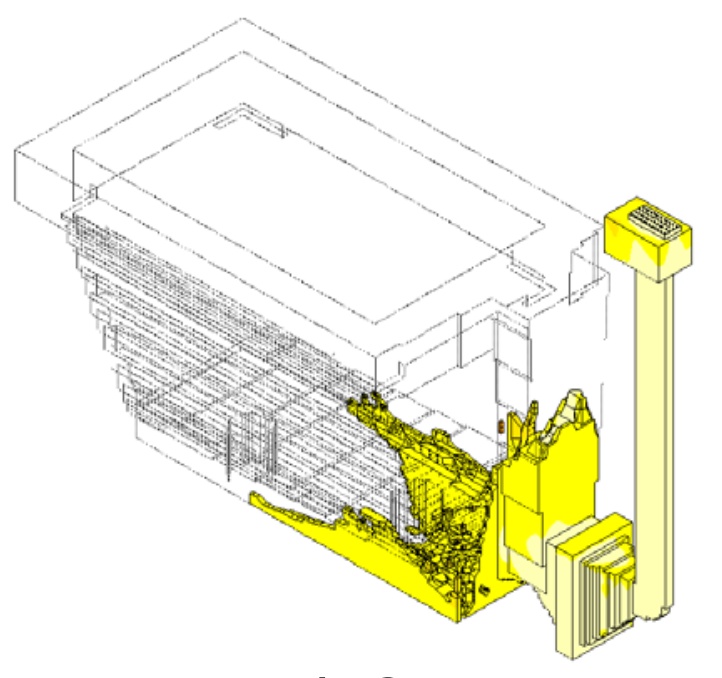

$1.2 \mathrm{sec}$

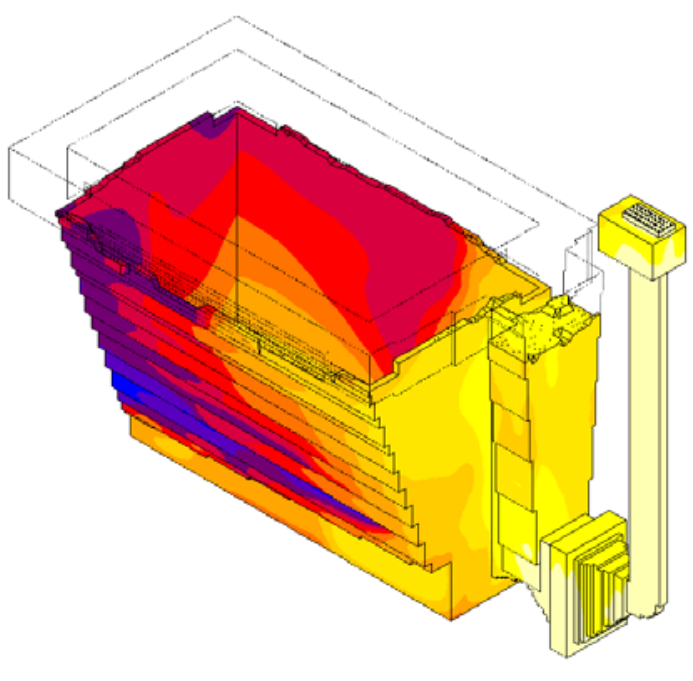

$6.0 \mathrm{sec}$

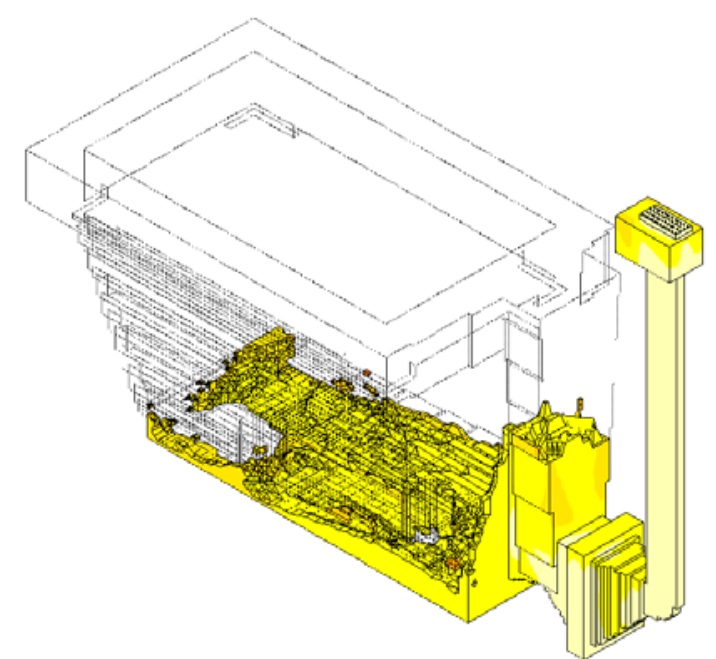

$1.6 \mathrm{sec}$

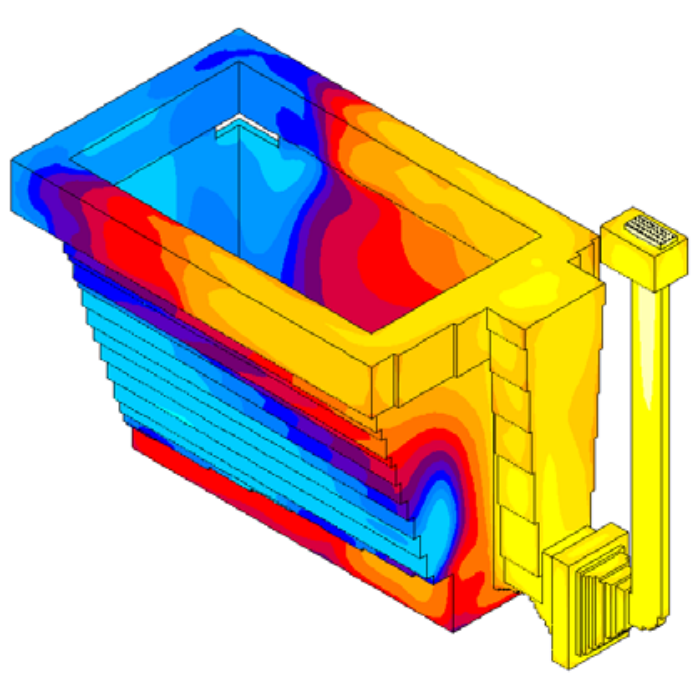

$8.0 \mathrm{sec}$

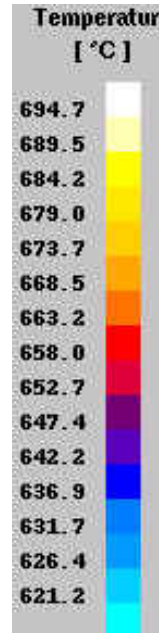

emperature

694.7

668.5

652.7

636.9 
Figure 61: Simulation Results of the "Box Casting" (c) Fraction Solid during Solidification

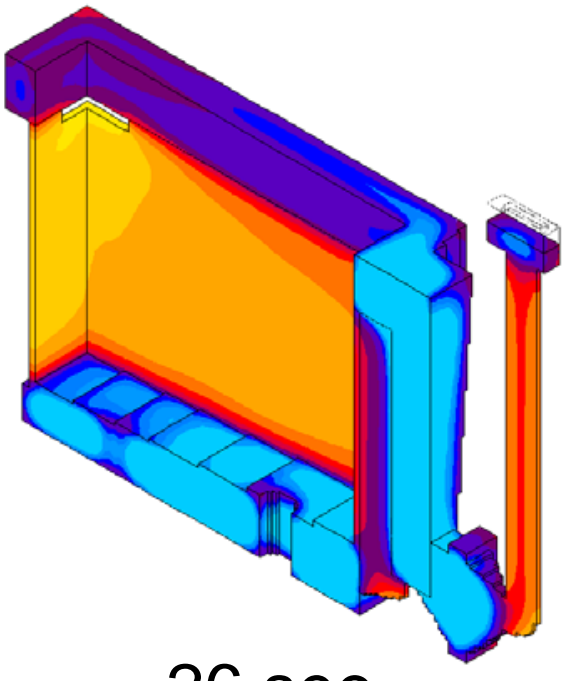

$26 \mathrm{sec}$

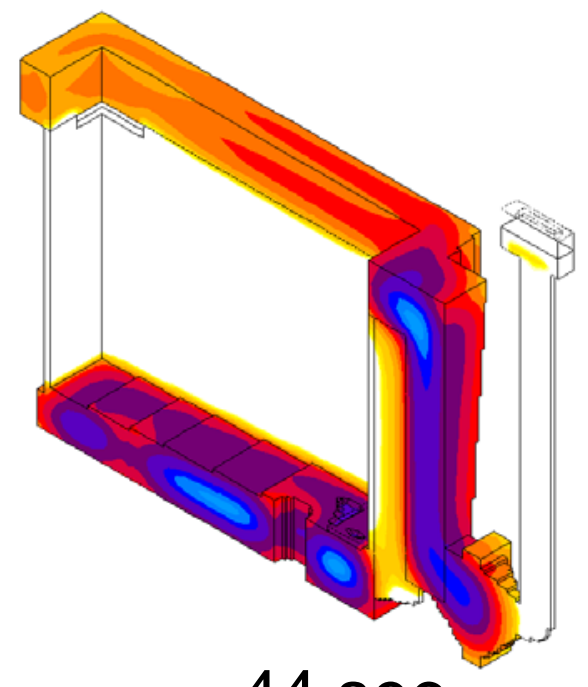

$44 \mathrm{sec}$

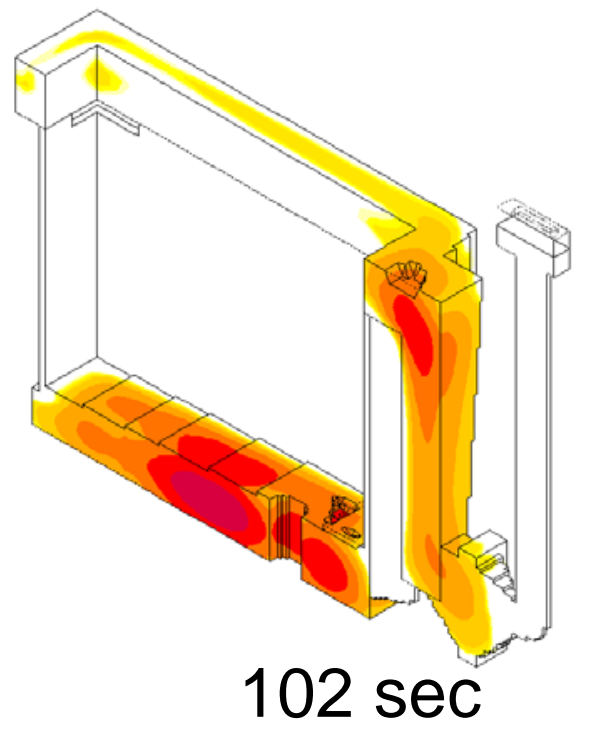

(b) Temperature Distribution during Solidification
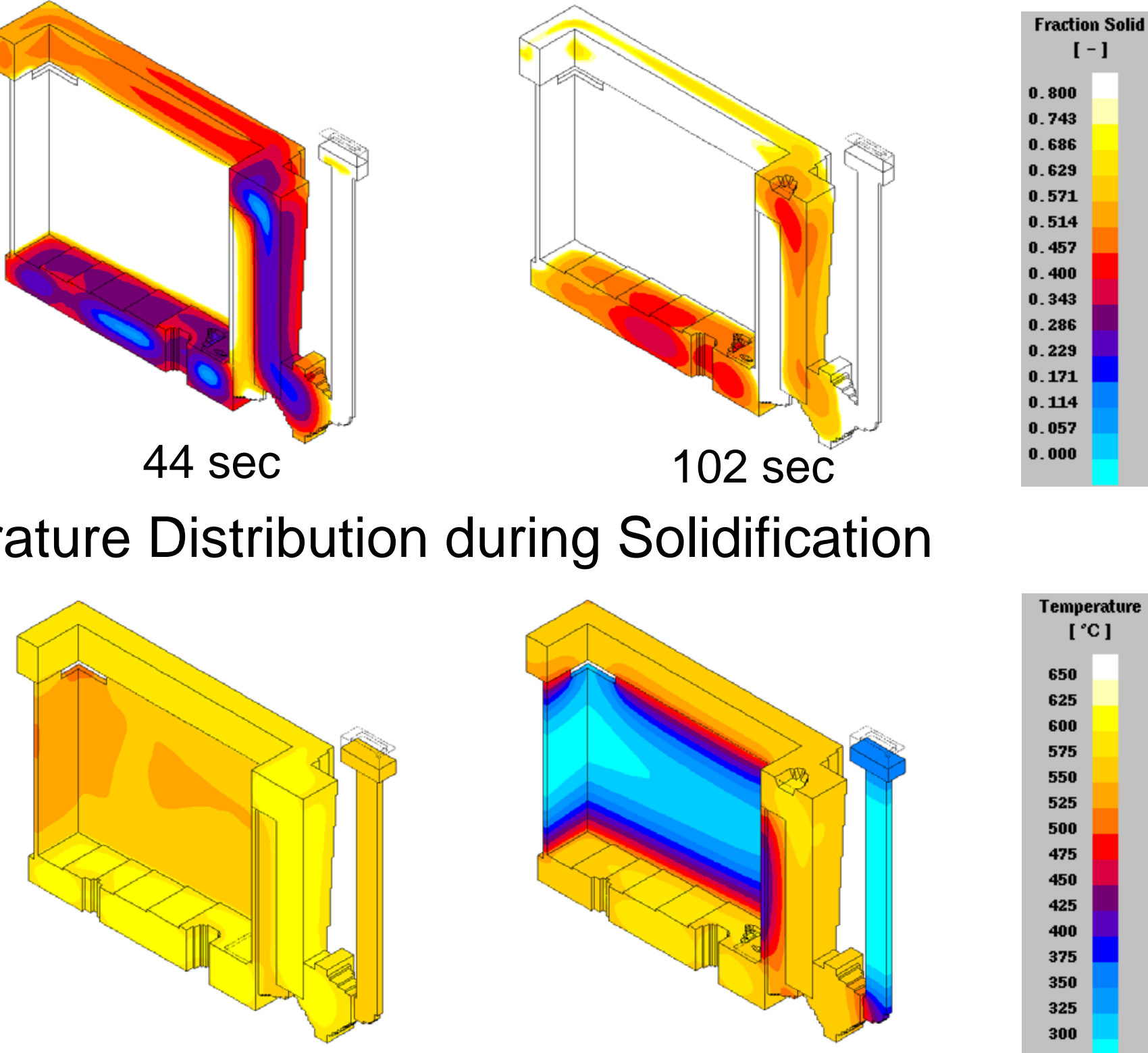

$44 \mathrm{sec}$

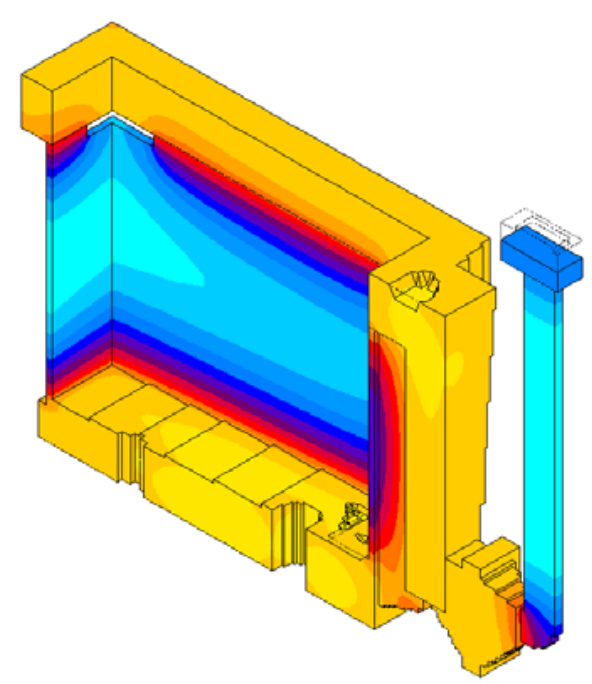

$102 \mathrm{sec}$ 
Figure 61 : Simulation Results of the "Box Casting"

(d) Feeding

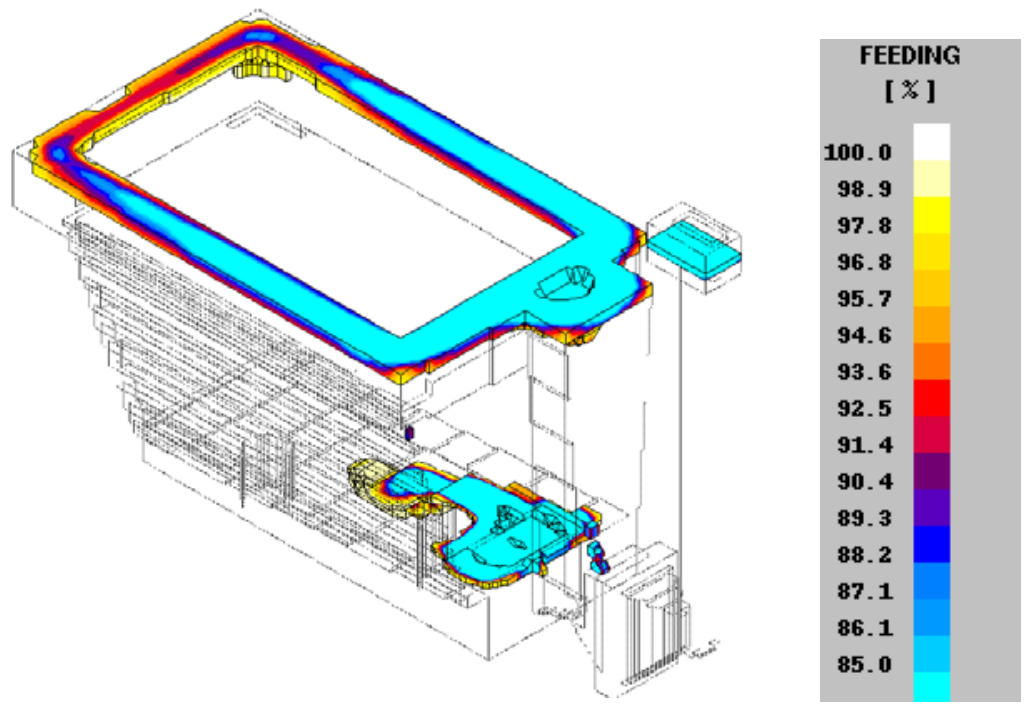

(b) Cooling Curve
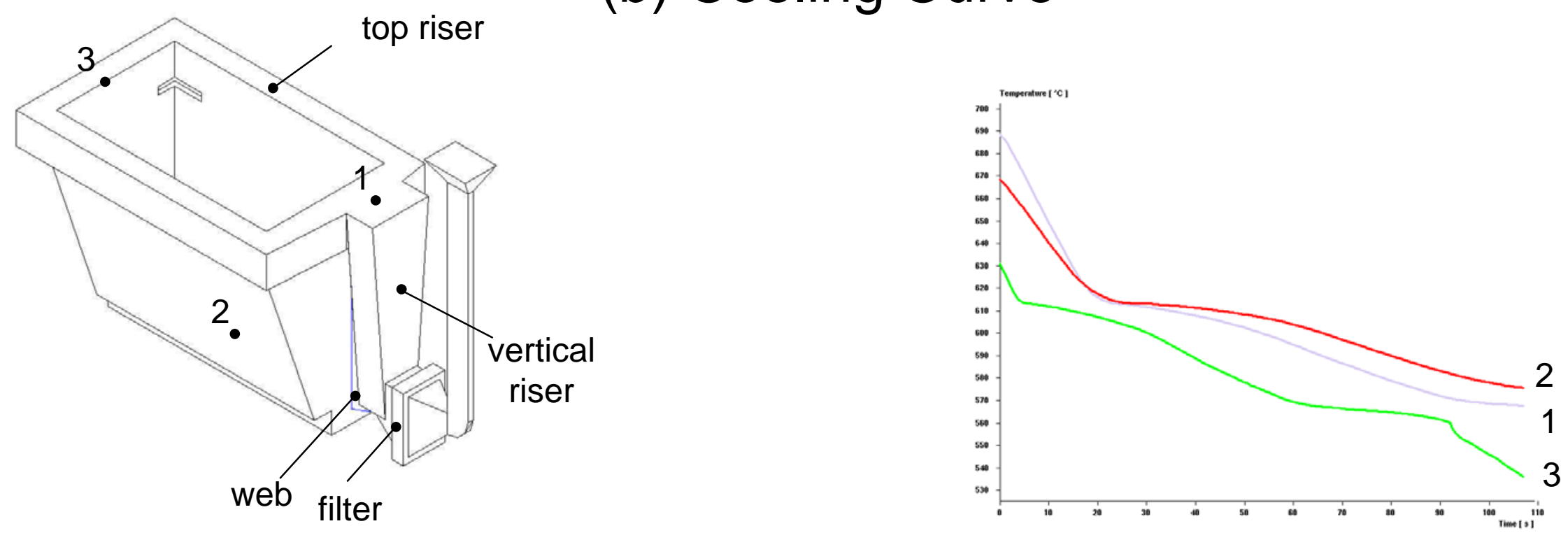
Figure $62 \mathrm{a}$ - Box Casting

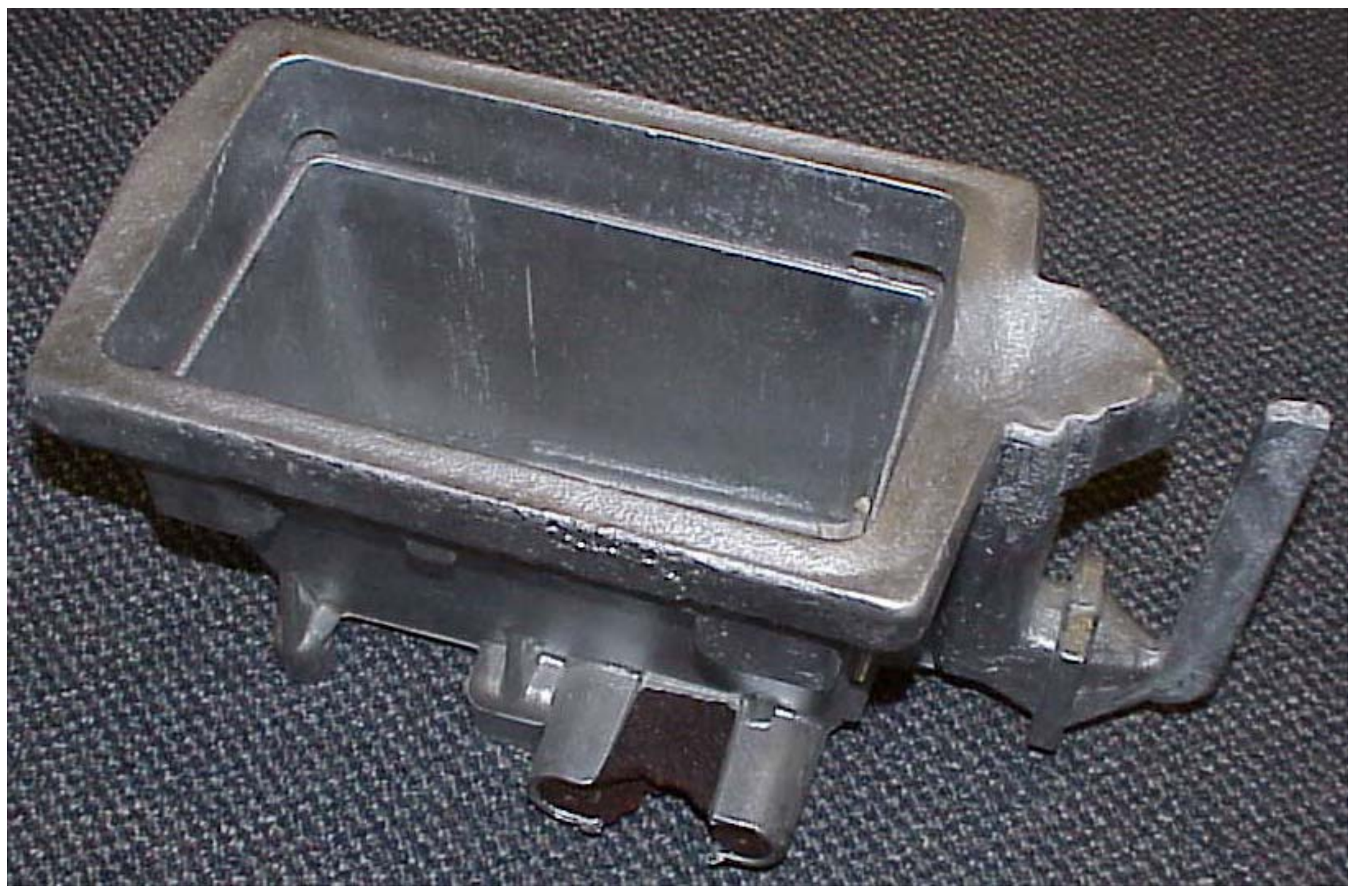




\section{Figure 62b - Box Casting}

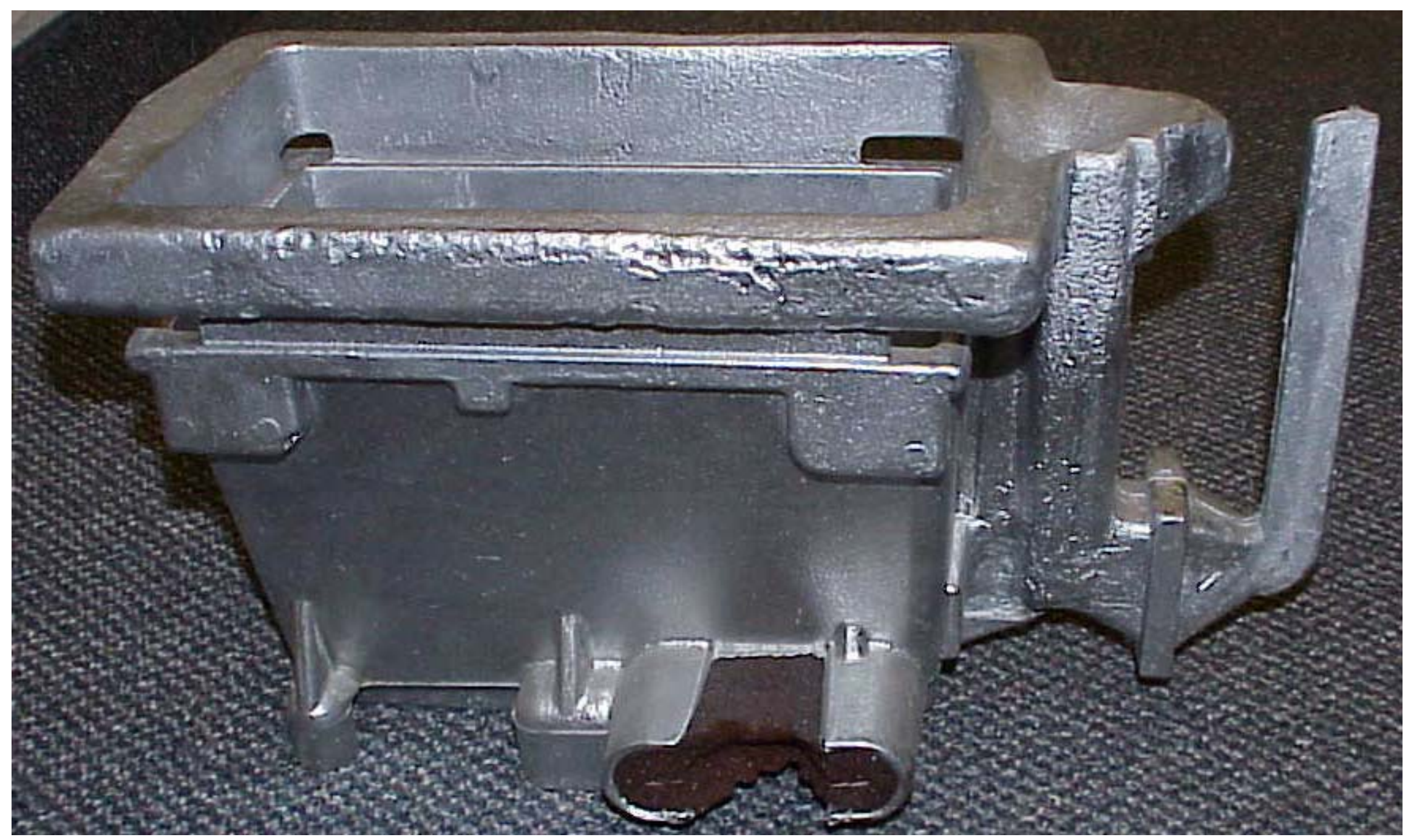


Figure 62c - Box Casting

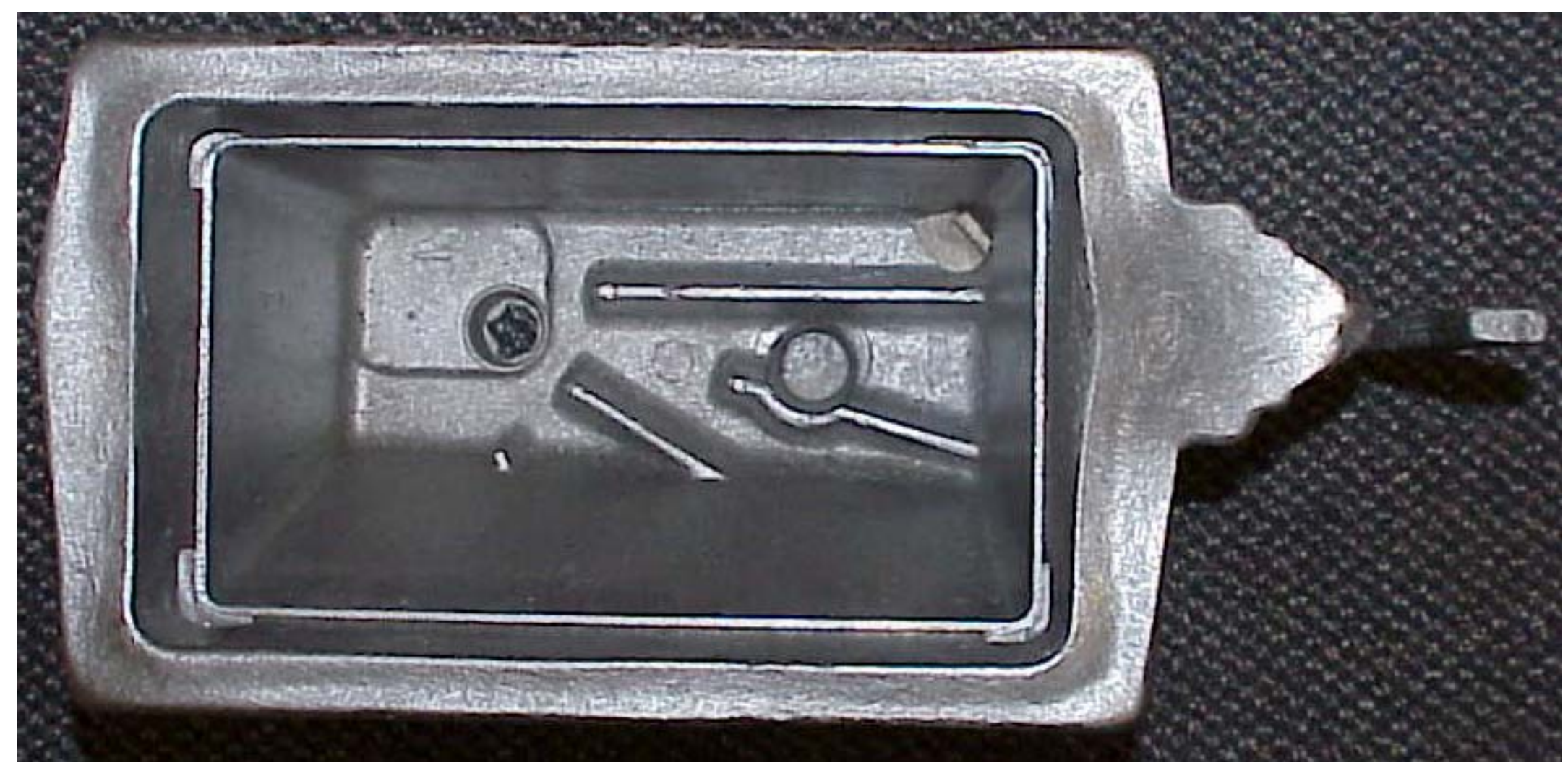


Figure 62d - Box Casting

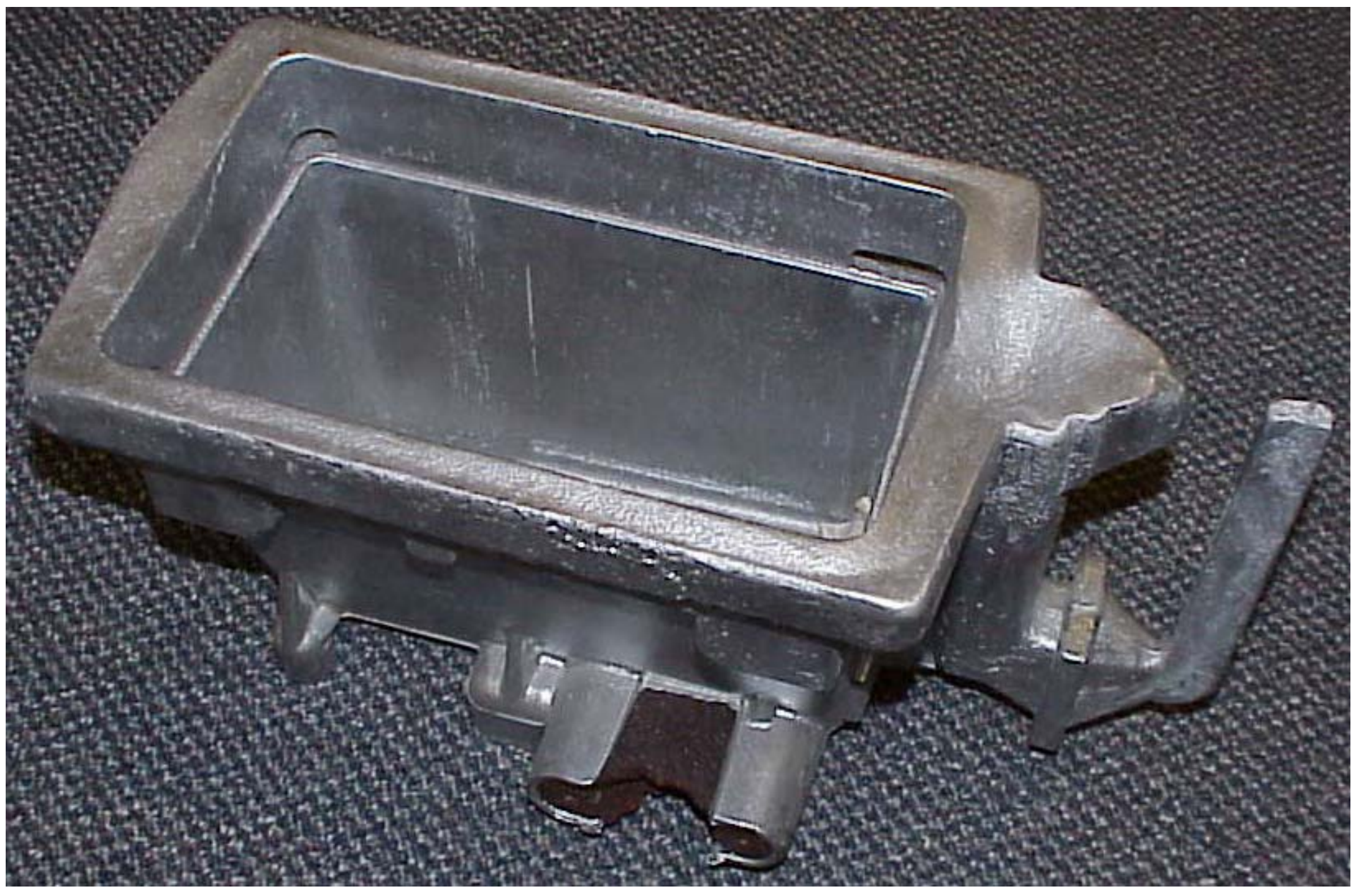




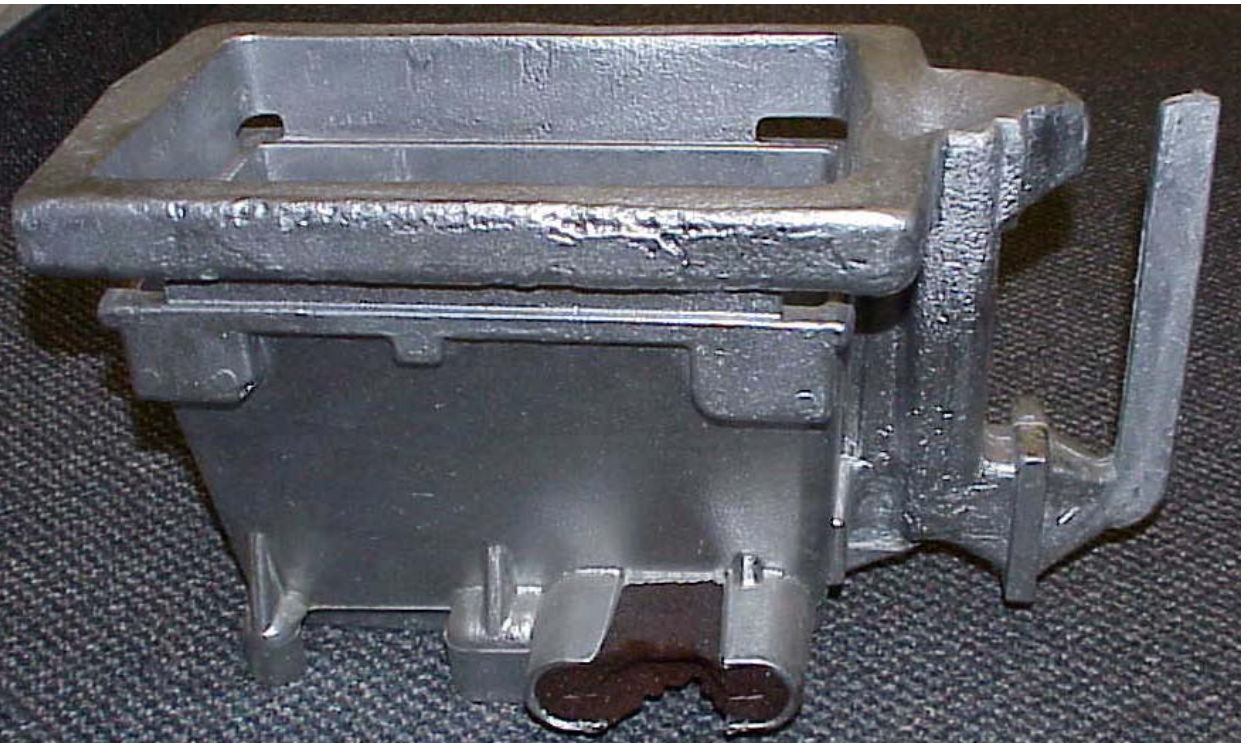

Figure 62e - Box Casting
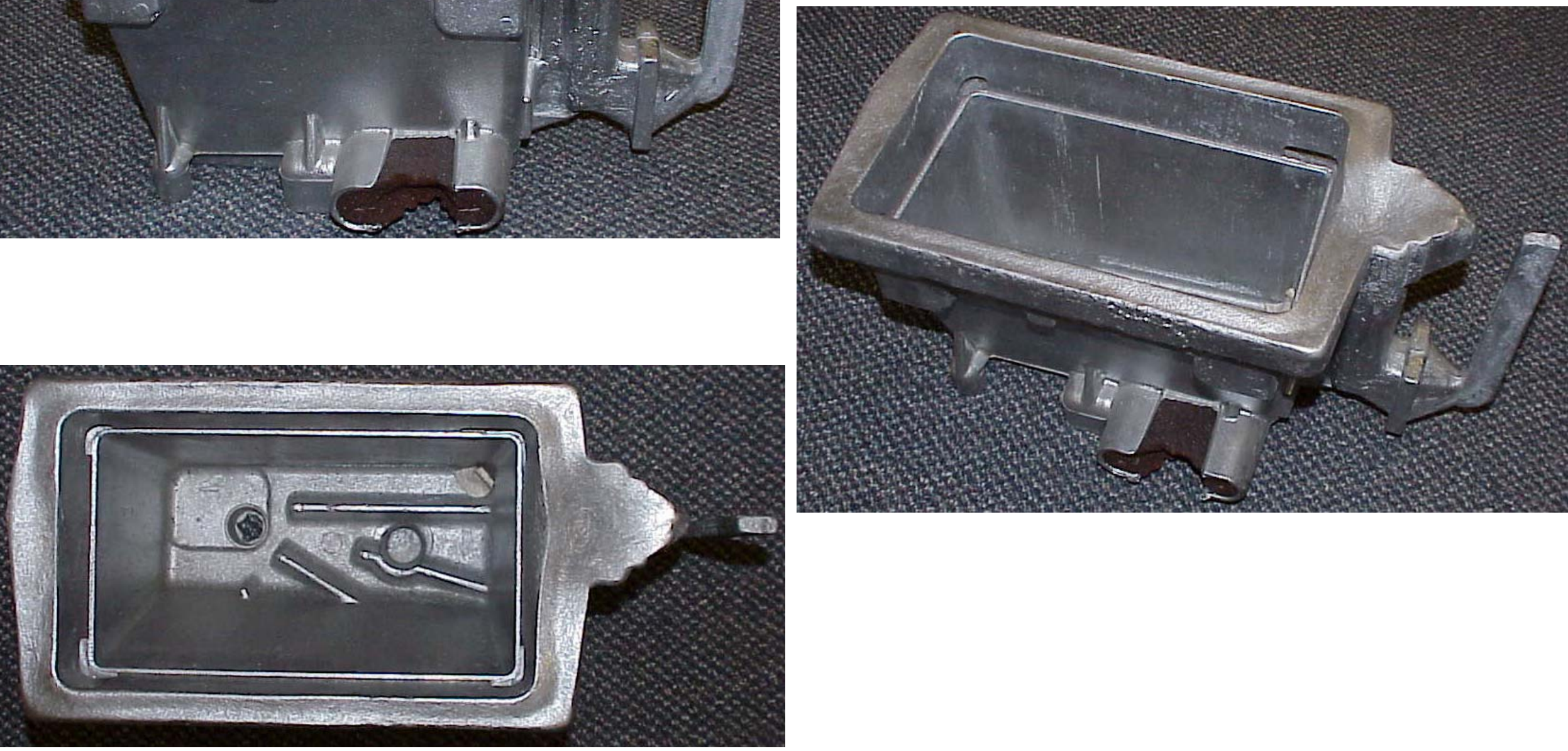
Area of Critical Sections

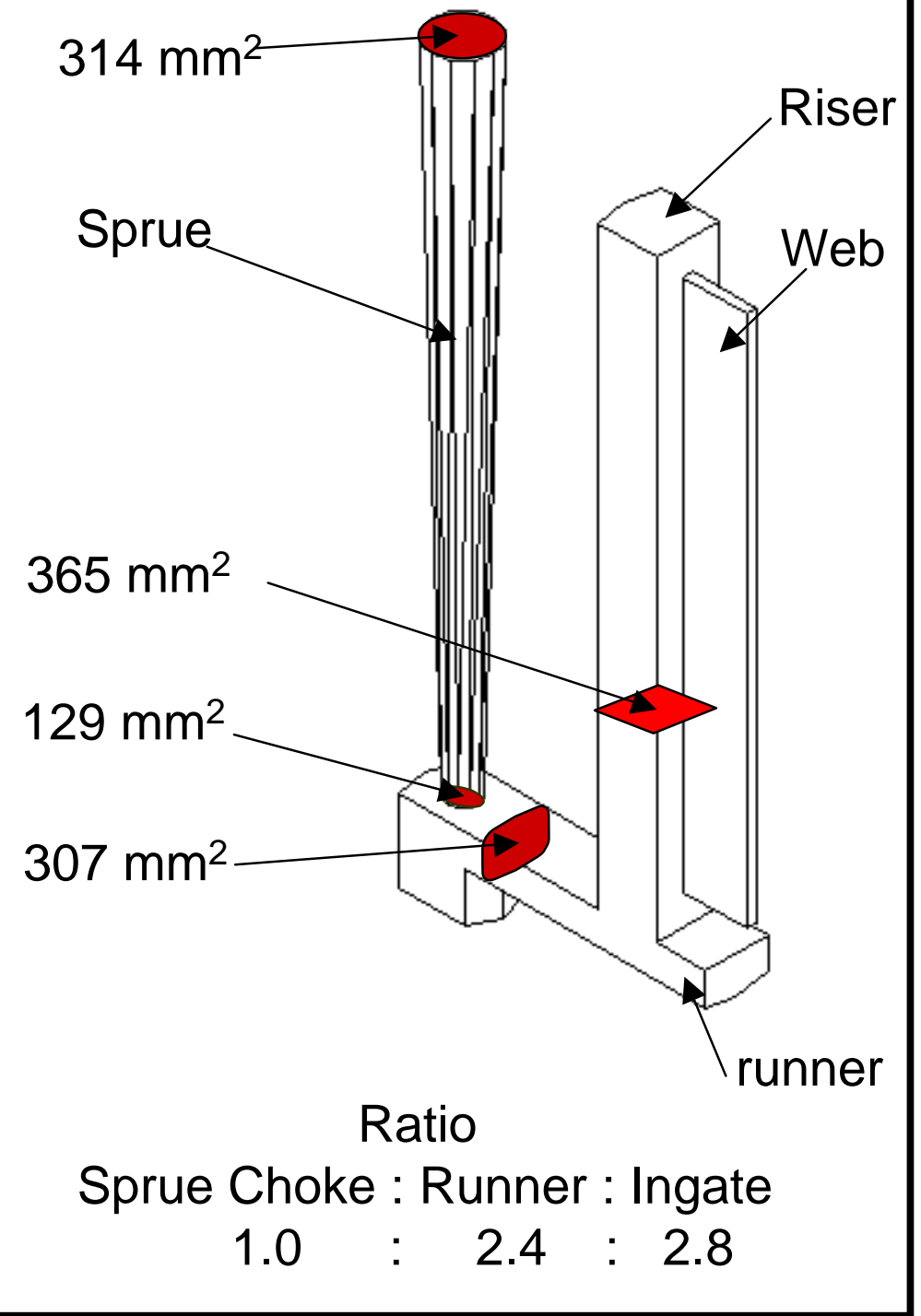

\section{Figure 63: MOLD 61}

- Web gating system with a large vertical riser and a thin web.

- Metal flows into the vertical riser first before cascading out of the web. 
Figure 64: Flow Pattern in mold 61, Web Thickness $=2.0 \mathrm{~mm}$

Molten Al356

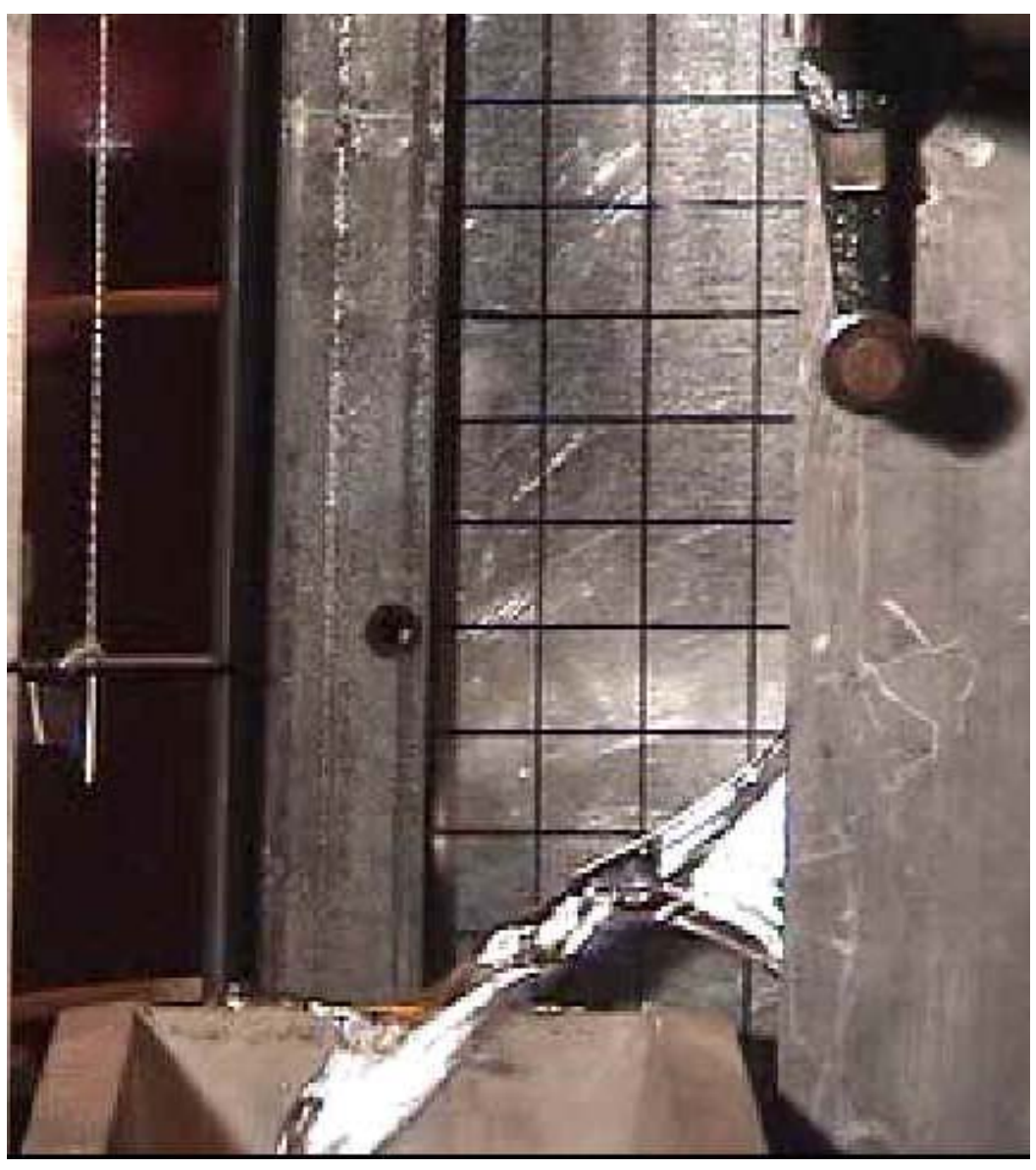

$1.246 \mathrm{sec}$
Water

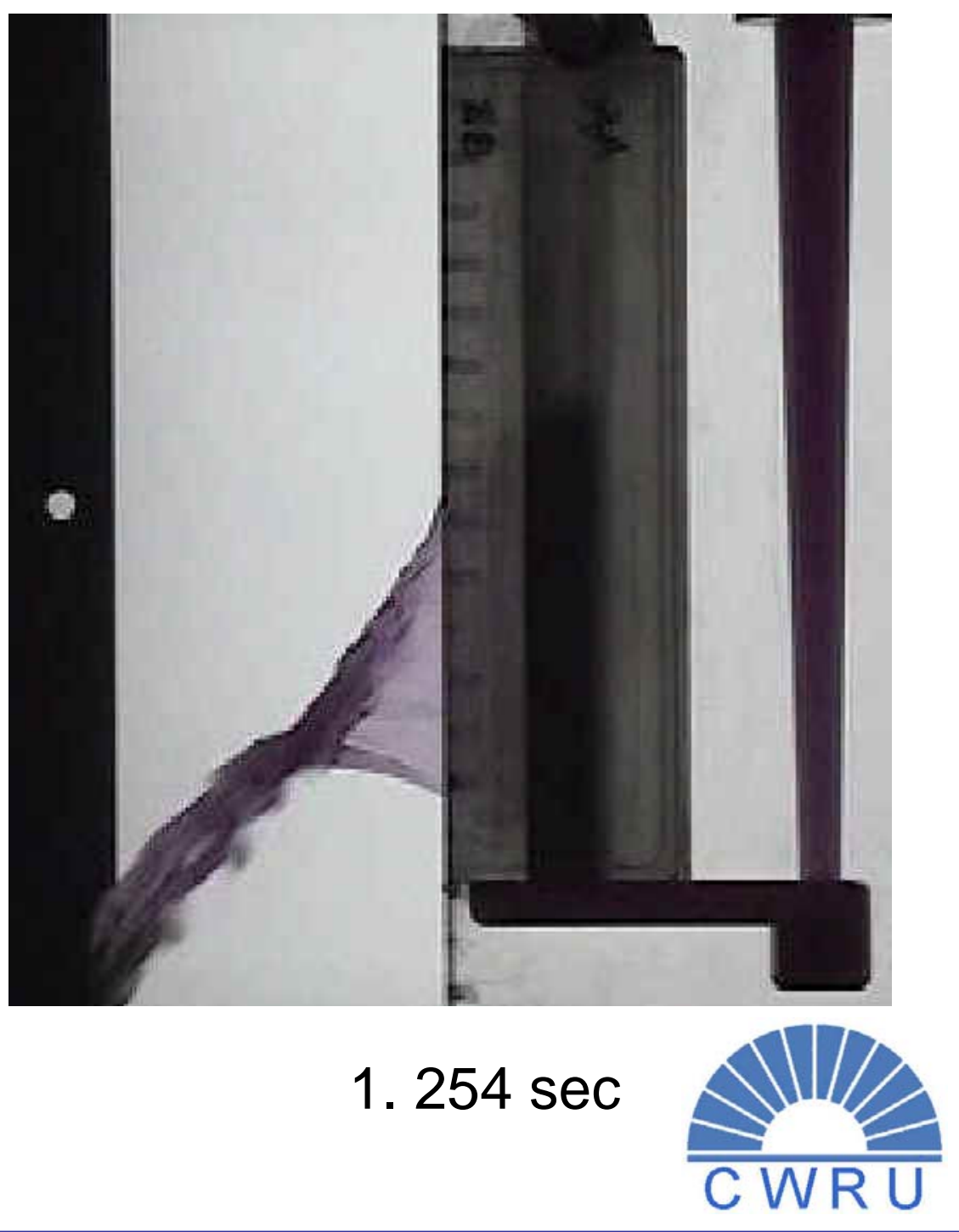


Figure 65: Horizontal Jet Distance vs. Web Thickness in a Webgated Vertical Mold with a Riser ( Molten Al356 )

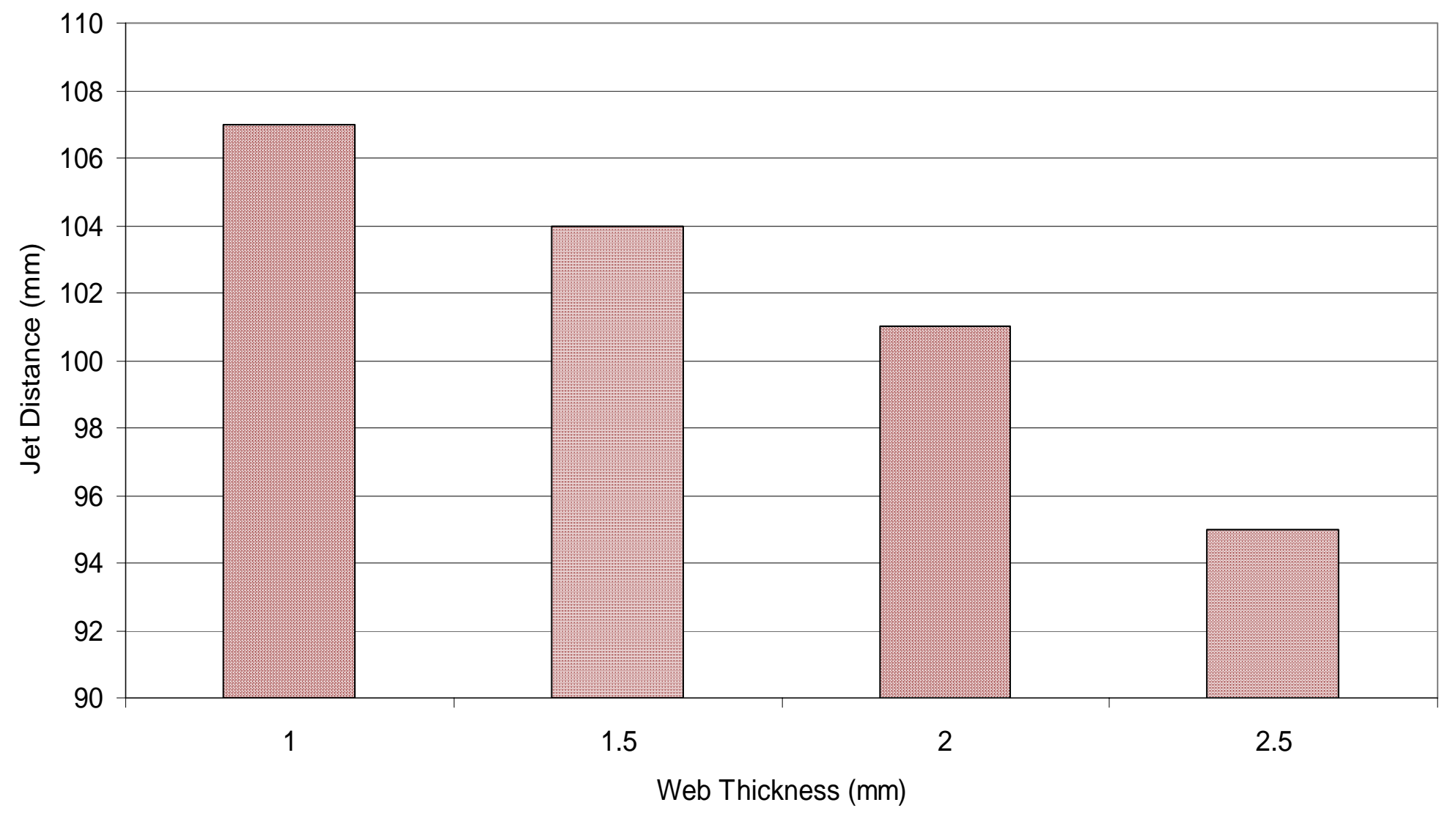

In the presence of a riser, jetting distance is not very sensitive to web thickness. Instead jetting more or less, the metal level in the riser increases or decreases depending on the web thickness.

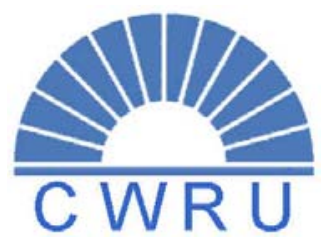


Figure 66: Maximum Water Height in the Riser vs. Web

Thickness in a Web-gated Vertical Mold ( Water )

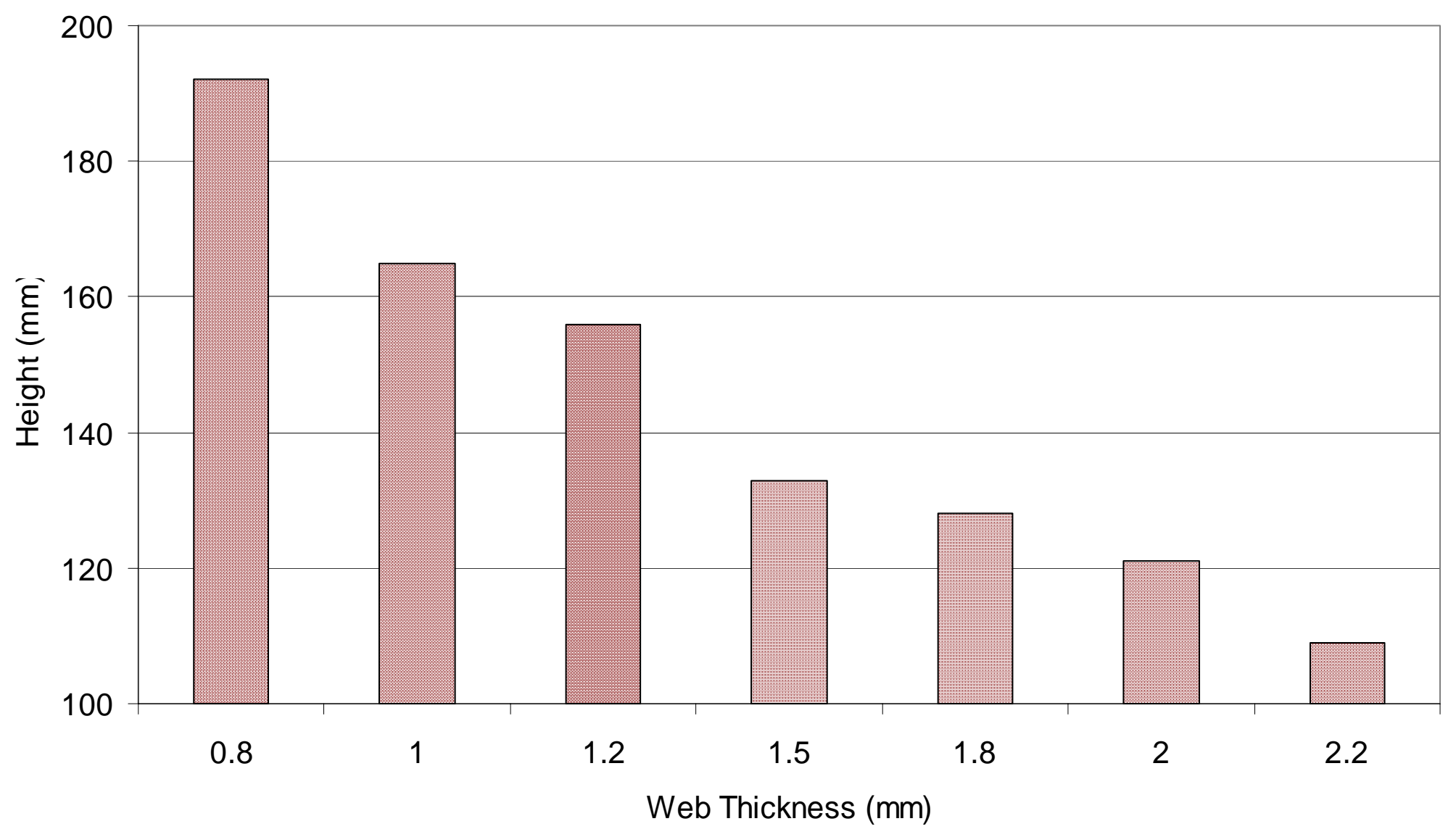

The water level in the riser is very sensitive to the web thickness 


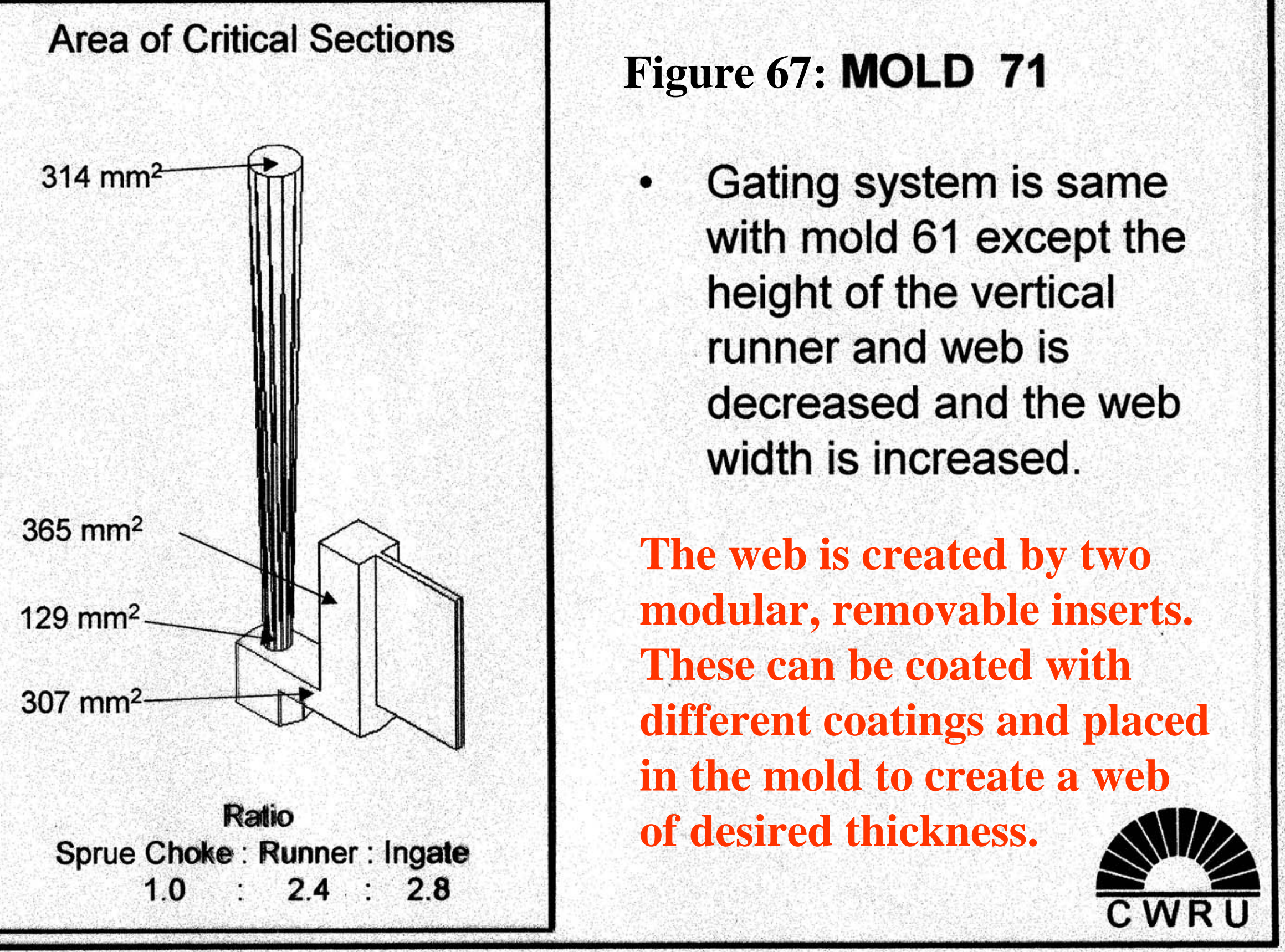




\section{Figure 68: EDGE - ON VIEW OF ESS 34 ROUGH COATING}

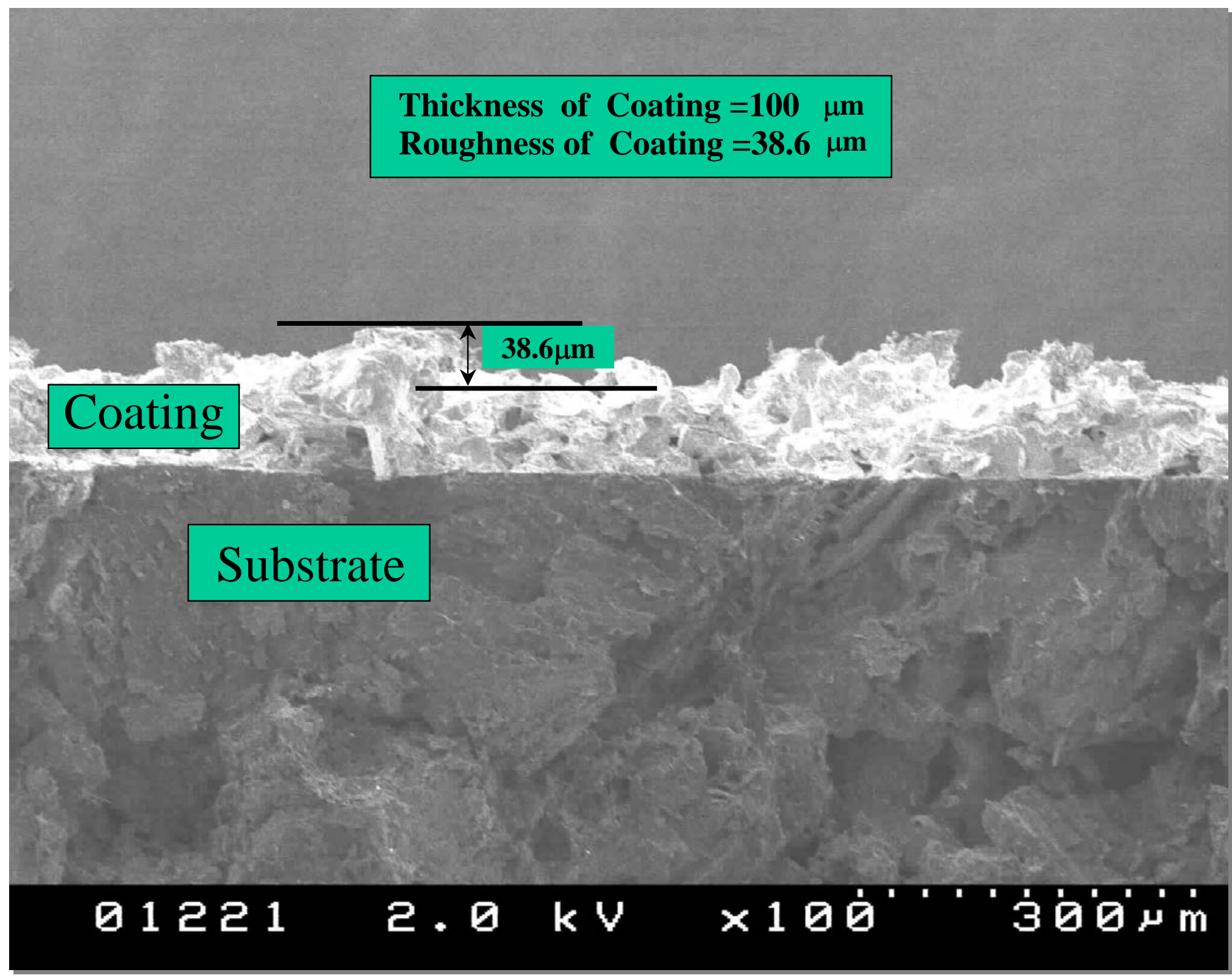




\section{Figure 70}

Horizontal Jet Distance vs. Time after Pouring Start in a Web-gated Vertical Mold ( web thickness $=1.3 \mathrm{~mm}$ )

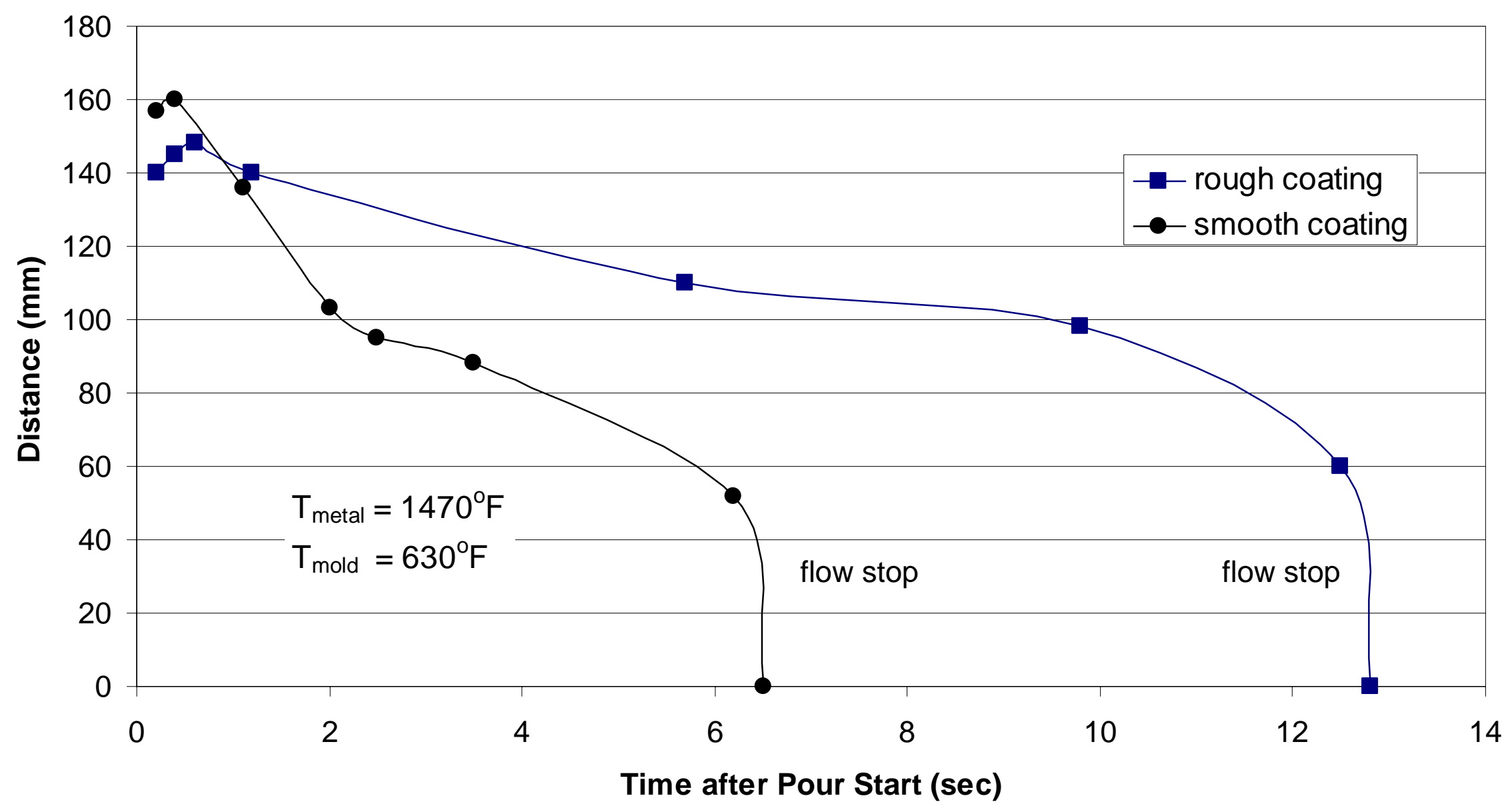


Figure 71: Flow of Aluminum out of a $1.3 \mathrm{~mm}$ Web
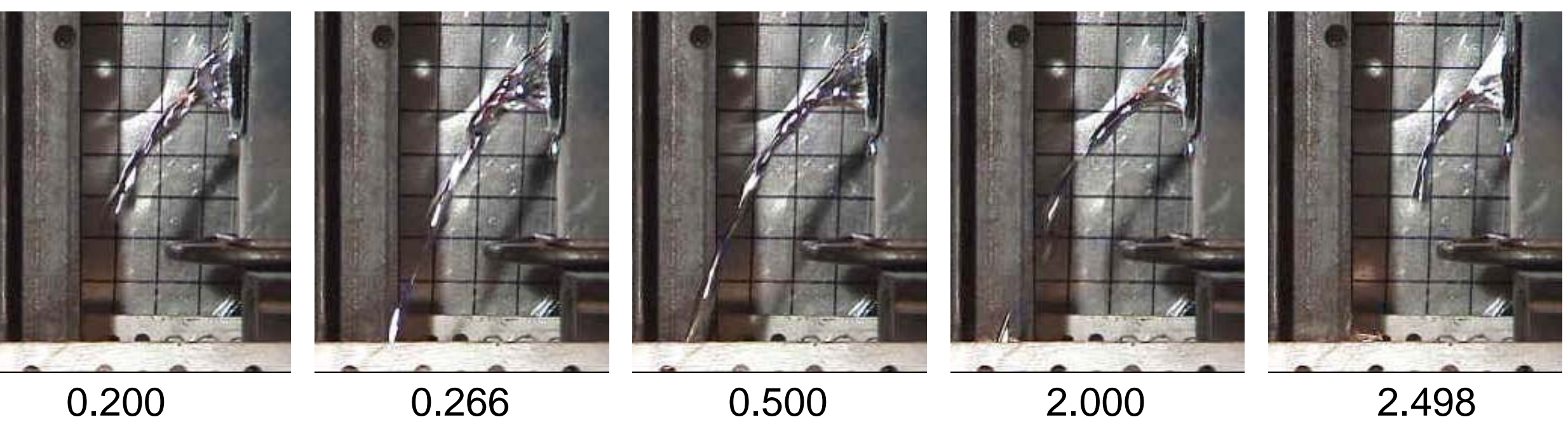

Rough Surface Insert

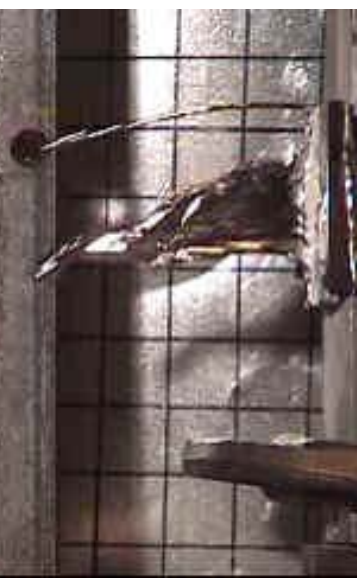

0.133

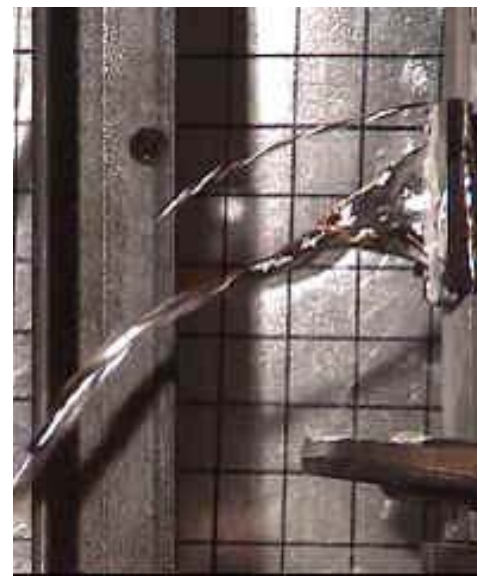

0.200

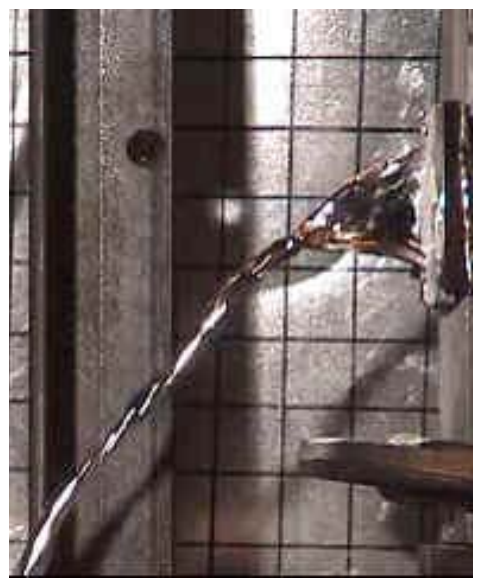

0.500

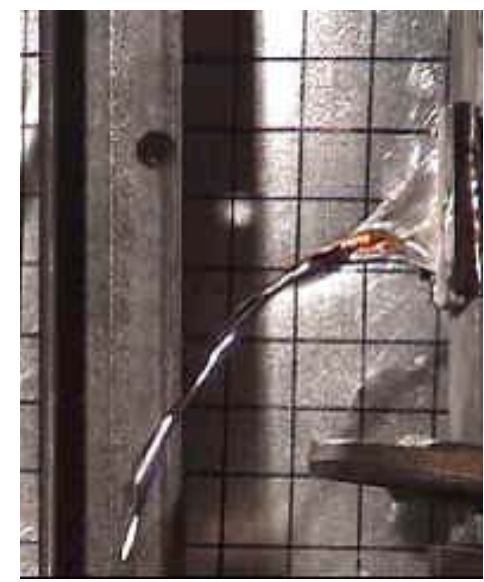

2.000

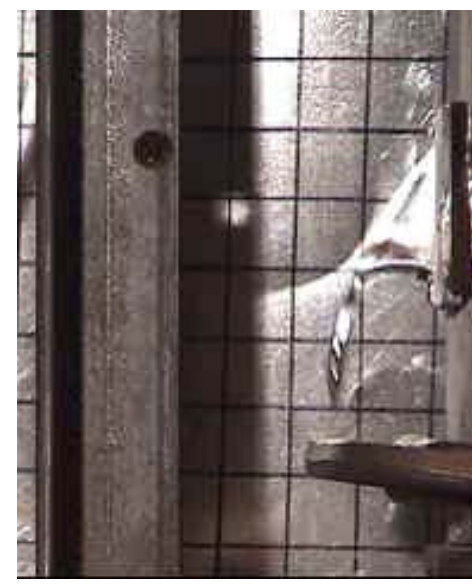

2.231

Smooth Surface Insert 
Figure 72

Horizontal Jet Distance vs. Time after Pouring Start in a Short Riser Web-gated Vertical Mold ( web thickness $=1.3 \mathrm{~mm}$ )

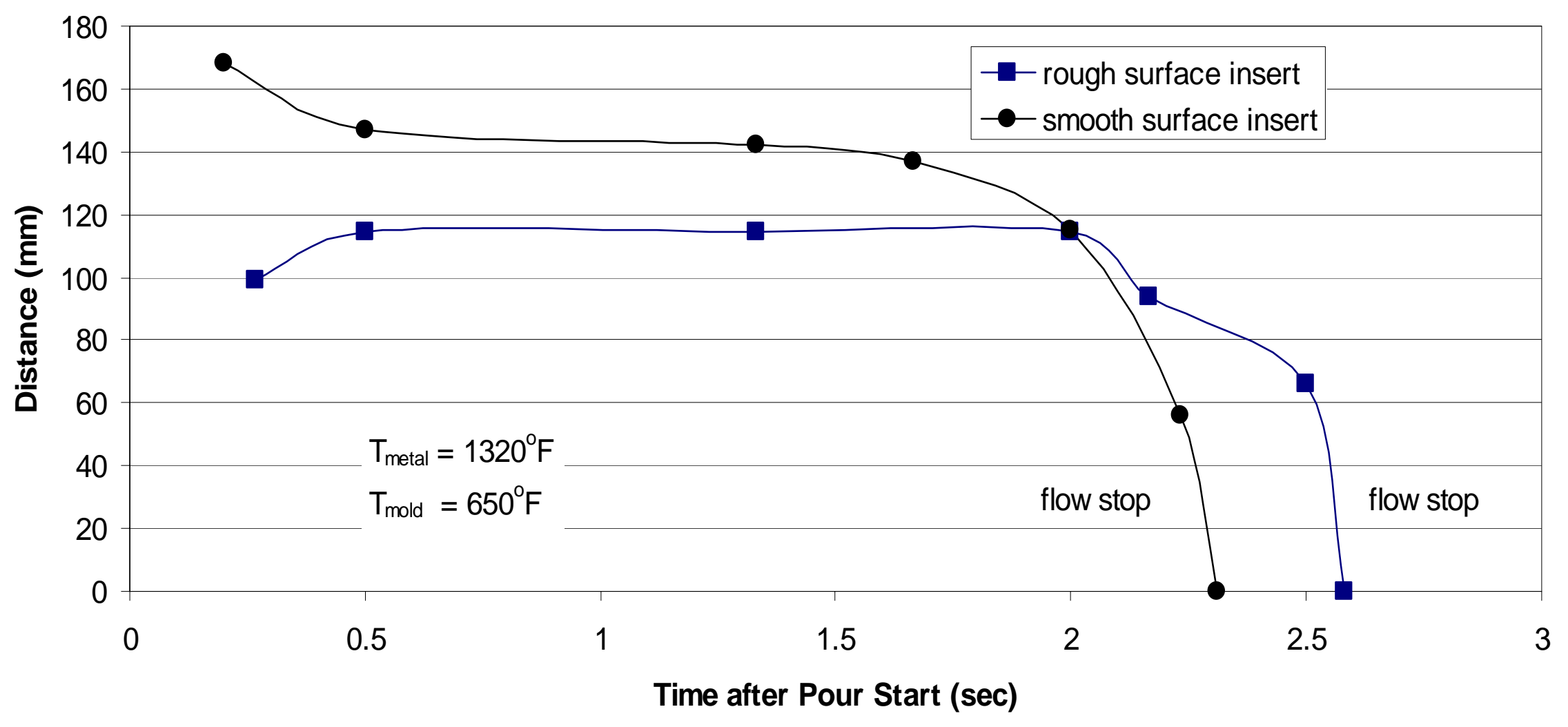

